\title{
Vector autoregressions
}

Citation for published version (APA):

Reuvers, H. (2018). Vector autoregressions: lag order uncertainty and least absolute deviations. [Doctoral Thesis, Maastricht University]. Datawyse / Universitaire Pers Maastricht. https://doi.org/10.26481/dis.20180514jr

Document status and date:

Published: 01/01/2018

DOI:

10.26481/dis.20180514jr

Document Version:

Publisher's PDF, also known as Version of record

\section{Please check the document version of this publication:}

- A submitted manuscript is the version of the article upon submission and before peer-review. There can be important differences between the submitted version and the official published version of record.

People interested in the research are advised to contact the author for the final version of the publication, or visit the DOI to the publisher's website.

- The final author version and the galley proof are versions of the publication after peer review.

- The final published version features the final layout of the paper including the volume, issue and page numbers.

Link to publication

\footnotetext{
General rights rights.

- You may freely distribute the URL identifying the publication in the public portal. please follow below link for the End User Agreement:

www.umlib.nl/taverne-license

Take down policy

If you believe that this document breaches copyright please contact us at:

repository@maastrichtuniversity.nl

providing details and we will investigate your claim.
}

Copyright and moral rights for the publications made accessible in the public portal are retained by the authors and/or other copyright owners and it is a condition of accessing publications that users recognise and abide by the legal requirements associated with these

- Users may download and print one copy of any publication from the public portal for the purpose of private study or research.

- You may not further distribute the material or use it for any profit-making activity or commercial gain

If the publication is distributed under the terms of Article $25 \mathrm{fa}$ of the Dutch Copyright Act, indicated by the "Taverne" license above, 
(C) J.W.N. Reuvers, Maastricht 2018

All rights reserved. No part of this publication may be reproduced, stored in a retrieval system, or transmitted in any form, or by any means, electronic, mechanical, photocopying, recording or otherwise, without the prior permission in writing from the author.

This book was typeset by the author using $\mathrm{LT}_{\mathrm{E} X}$.

Cover design by Luca Baseggio and Caterina Schiavoni

Published by Universitaire Pers Maastricht

ISBN: 978-94-6295-922-4

Printed in The Netherlands by Datawyse Maastricht

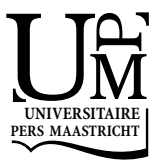




\title{
Vector Autoregressions: Lag Order Uncertainty and Least Absolute Deviations
}

\author{
DISSERTATION \\ to obtain the degree of Doctor at \\ Maastricht University, \\ on the authority of the Rector Magnificus, \\ Prof. dr. R.M. Letschert, \\ in accordance with the decision of the Board of Deans, \\ to be defended in public \\ on Monday, $14^{\text {th }}$ of May 2018, at 14:00 \\ by \\ Johannes Wilhelmus Nicolaas Reuvers
}




\section{Supervisors}

Prof. dr. F.C. Palm

Prof. dr. J.R.Y.J. Urbain (†)

Dr. S.J.M. Smeekes (co-promotor)

\section{Assessment Committee}

Prof. dr. A.W. Hecq (Chair)

Dr. E. Beutner

Prof. dr. C. Croux (EDHEC Business School)

Prof. dr. S.J. Koopman (Vrije Universiteit Amsterdam)

This research was financially supported by The Netherlands Organisation for Scientific Research (NWO). 
To Marie-Thérèse, Nico and Nico.

Dedicated to the memory of Jean-Pierre Urbain 



\section{Acknowledgements}

"Knowledge is in the end based on acknowledgement."

- Ludwig Wittgenstein (1889-1951)

Thank you! While starting to write these acknowledgements I could not think of any better starting words than these. I do not know which eyes are currently reading these lines but the mere fact that you are reading them indicates that our lives have crossed at one point or another. For this simple fact I would like to express my gratitude irrespectively of you being a friend, a colleague or any other person that has been helpful these last years. I will not try the full enumeration of all of you because I know that I will surely forget someone.

However, there are several people that I have to mention explicitly. First of all, there are my supervisors Franz Palm and Jean-Pierre Urbain. My PhD would have never started if you had not given me this opportunity. Additionally, my thesis would not have been completed without your excellent guidance. You were the captains that navigated my $\mathrm{PhD}$ ship to a safe haven. Both your knowledge and your inspiration have guided me through the storms that occasionally clouded the journey towards my dissertation. I learned a lot from you, not only on econometric topics but also from the nice persons you are and have been.

A defense cannot take place without the consent of the reading committee. I therefore thank Eric Beutner, Christophe Croux, Alain Hecq and Siem Jan Koopman for their willingness to form this committee and their efforts to assess my thesis manuscript. Let me also not forget to extend my gratitude to Stephan Smeekes for being my co-promotor. I am grateful to Joakim Westerlund for hosting me for three months at Lund University. Also, a big 'merci' to the two wonderful secretaries of the QE department: Karin van den 
Boorn and Yolanda Paulissen. And finally, there are several persons with whom I was tutoring courses. I enjoyed teaching with you. Some of you have been mentioned already in this paragraph. Denis de Crombrugghe is mentioned explicitly because of his contagious passion for education.

On a more personal level I turn to Artem, Etiënne, Yuliya and Yicong. I feel blessed with your friendship and I am deeply indebted for your presence around me. It is a cruel fact that only two people can be appointed as paranymphs and choosing between the four of you was one of the hardest problems of my $\mathrm{PhD}$. Only two names can appear in the official documents and only two persons can stand at my side at the time of my defense. However, in my mind there will always be four.

Finally, there are my closest relations. Papa, thanks for your good cares and calming advices. You must have awakened my interest in mathematics while asking me to calculate the bill for groceries. Mama, thanks for always being around to take care of everything. I still cannot understand how you manage to remind me of all the daily life things I tend to forget. It has been several years since someone thanked his 'broertje' in his acknowledgments. Right now, it is my turn to make that remark. Nico, 'broertje', I am fortunate to have an older brother like you to lead the way. Thanks for your knowledge and humor. Caterina, the very last sentences on this page are dedicated to you. Your liveliness provides me with fresh energy after a tiring day of work, seeing your smile always puts me into a good mood and your encouragements helped me through several stressful times. Thanks for everything.

Hanno Reuvers

Maastricht, April 2018 


\section{Contents}

1 Introduction 1

1.1 What to expect from this thesis? . . . . . . . . . . . . 1

1.2 What not to expect from this thesis? . . . . . . . . . . . 4

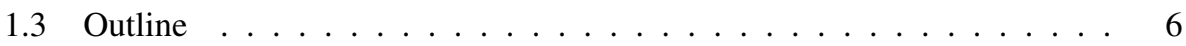

1.4 Notation ........................... 6

2 A Focused Information Criterion for Locally Misspecified Vector Autore$\begin{array}{ll}\text { gressive Models } & 7\end{array}$

2.1 Introduction . . . . . . . . . . . . . . . . 9

2.2 Theory ................................ 11

2.2.1 The Model Framework . . . . . . . . . . . . . . . . 11

2.2.2 Parameter Estimation and Asymptotics . . . . . . . . . . . . 11

2.2.3 The Quantities of Interest . . . . . . . . . . . . . . . . 16

2.2.4 The Effects of Inconsistently Estimating Delta . . . . . . . . . 22

2.3 Simulation Results _ . . . . . . . . . . . . . . . . 26

2.3.1 The simplified DGP . . . . . . . . . . . . . . . . 27

2.3.2 Simulation Results for an Autoregressive Model . . . . . . . . . 27

2.3.3 Simulation Results for a Vector Autoregressive Model . . . . . . 31

2.4 Conclusion . . . . . . . . . . . . . . . . . . . 39

2.A Mathematical Proofs . . . . . . . . . . . . . . 40

2.B Derivation of the Optimal Weights in the Simplified Model . . . . . . . . 48 
2.C No Gradient Dependence in Simplified Model . . . . . . . . . . . . . . . 49

2.D Simulations for Multiple Focus Parameters . . . . . . . . . . . . . 51

3 Estimating VAR models by Least Absolute Deviations $\quad 53$

3.1 Introduction . . . . . . . . . . . . . . . . 55

3.2 The Estimator . . . . . . . . . . . . . . . . . 56

3.2.1 Asymptotic Properties of the LAD Estimator . . . . . . . . . . 59

3.2.2 Hypothesis Testing . . . . . . . . . . . . . . . 65

3.2.3 Determination of the Lag Order . . . . . . . . . . . . . 68

3.3 Simulation Results . . . . . . . . . . . . . . . . . . . . 69

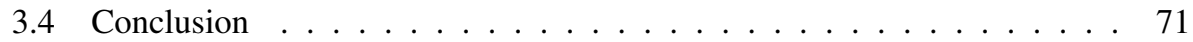

3.A Appendix ........................... 84

3.A.1 Implementation of the Linear Program . . . . . . . . . . . . . . . 84

3.A.2 Proof of Theorem 1: Consistency . . . . . . . . . . . . . 84

3.A.3 Proof of Theorem 2: Asymptotic Normality . . . . . . . . . . . 89

3.A.4 Proof of Theorem 3: Consistent Estimation of the Asymptotic Variance . . . . . . . . . . . . . 95

3.A.5 Proof of Theorem 4-6: Test Statistics _ . . . . . . . . . . . 99

3.A.6 Proof of Theorem 7: Consistent Selection of the Lag Order . . . . 102

3.B Supplementary Material . . . . . . . . . . . . . . . . . . . . . 104

3.B.1 Huber's (1967) Lemma for Dependent Processes . . . . . . . . . 104

3.C Supplementary Simulation Results . . . . . . . . . . . . . . . . . . . 110

3.C.1 Histograms for Larger Sample Sizes . . . . . . . . . . . . . . . . 110

3.C.2 Information Criteria for Larger Sample Sizes . . . . . . . . . . . 111

4 Residual Bootstrap for VAR Models Estimated by Least Absolute Deviations 113

4.1 Introduction . . . . . . . . . . . . . . . . . 115

4.2 Theory . . . . . . . . . . . . . . . . . . . 117

4.2.1 The Model . . . . . . . . . . . . . . . . . . . 117

4.2.2 The Bootstrap . . . . . . . . . . . . . . . . 119

4.3 Simulation Results . . . . . . . . . . . . . . . . . . . . . 124

4.4 Conclusion ........................... 126 
4.A Mathematical Appendix . . . . . . . . . . . . . . . . 130

4.A.1 Parameter Estimation . . . . . . . . . . . . . . . . 130

4.A.2 Hypothesis Tests . . . . . . . . . . . . . . . . 136

4.B An MC Comparison with Unrestricted Residuals . . . . . . . . . . . . 139

4.C An MC Comparison with a Bootstrap Method with Recursive Design . 140

4.D Simulation Results on Median Resampling . . . . . . . . . . . . . . . . . 144

$\begin{array}{lll}5 & \text { Conclusion } & 147\end{array}$

5.1 Concluding Remarks on the Individual Chapters . . . . . . . . . . . . . . 147

5.2 General Conclusions . . . . . . . . . . . . . . . . . . . 148

$\begin{array}{ll}\text { Bibliography } & 151\end{array}$

$\begin{array}{ll}\text { Valorisation } & 162\end{array}$

$\begin{array}{ll}\text { Nederlandse Samenvatting } & 167\end{array}$

$\begin{array}{lr}\text { Curriculum Vitae } & 169\end{array}$ 



\section{Chapter 1}

\section{Introduction}

"What we call the beginning is often the end. And to make an end is to make a beginning. The end is where we start from." - T.S. Eliot (1888-1965)

The core chapters of this thesis cover three different topics. As such, you may read these chapters in any arbitrary order or simply select the chapter that is closest to your interest. There is no continuous story line but there is one commonality, namely the model. The Chapters 2-4 all focus on vector autoregressions.

The next pages will briefly introduce these vector autoregressions and we will raise and motivate the questions that will be (and will not be) addressed in this thesis. Along this path we will encounter various literature references that place this thesis into the context of the past and current research. The subsequent part of this introduction is an outline of the thesis you just now started reading. We end with some words on notation.

\subsection{What to expect from this thesis?}

An autoregressive model is a time series model in which past values have a linear impact on the current value. If we denote the time series by $\left\{y_{t}\right\}$, then

$$
y_{t}=\alpha y_{t-1}+u_{t},
$$

would probably be the easiest example. Equation (1.1) expresses the current value $y_{t}$ in terms of its past value $y_{t-1}$. The linear relationship $y_{t}=\alpha y_{t-1}$ never holds exactly so a ran- 
dom and unobserved innovation $u_{t}$ is introduced to account for these deviations. While retaining the linear structure, we can still extent this model by including (1) additional variables and/or (2) value of the variable(s) that go further back in time. In doing so, we arrive at a vector autoregression of order $p$ (abbreviated as $\operatorname{VAR}(p))$ in $K$ variables. ${ }^{1}$ In this $\operatorname{VAR}(p)$, every variable depends linearly on $p$ lags of all the variables in the system, i.e. the past $p$ values of the other $(K-1)$ variables and the past $p$ values of the series itself. These vector autoregressive models, advocated by Sims (1980), have been studied in depth because of their broad applicability. Examples from various fields of research are: a climatology study on the temperature relations between the Northern and Southern hemisphere by Kaufmann and Stern (1997); a study on directed interactions between activated brain areas by Goebel et al. (2003) in the field of neurophysiology; a financial application by Cochrane (2007) on return predictability; and a macro-economical review article by Stock and Watson (2001). This last reference on macro-economics is just a single draw out of many possible references since vector autoregressions have become a standard tool in macro-economics. The importance/popularity of vector autoregressive models is also evident from the large collection of econometric textbook that devote attention to this class of models. An extensive (but by no means an exhaustive) list of such econometric textbooks is Brillinger (2001), Fuller (1996), Gourieroux and Monfort (1997), Hamilton (1994), Hannan (1970), Hayashi (2000), Lütkepohl (2005) and Lütkepohl and Krätzig (2004). These textbooks describe how vector autoregressions can be used for a variety of tasks such as forecasting, impulse response analysis, and Granger causality testing.

Let us now assume that the data has been generated by a vector autoregression of lag order $p .^{2}$ This true lag order is usually unknown so a researcher has to determine a lag order to be used in the analysis. This chosen $p$ is important because an underestimation of the lag order leads to biased estimates whereas an overestimation unnecessarily decreases the precision of the estimates. The methods for lag length determination can be divided into three groups. We now provide a small overview of these methods and refer the reader to Chapter 4 of Lütkepohl (2005) and the paper by Nielsen (2006) for further details.

The most intuitive way to decide on the lag order is based on sequential testing. One starts with some a priori maximum lag length, say $p_{\text {max }}$, and tests whether all $K^{2}$ parameters in front of the $p_{\max }$ 'th lag are zero. A rejection of this hypothesis fixes the lag

\footnotetext{
${ }^{1}$ We could extent even further and allow deterministic components and/or exogenous variables to enter, but such extensions are not necessary within the scope of this thesis.

${ }^{2}$ Note the implicit assumption that the true model is a vector autoregression of finite order. We take this assumption for granted and implicitly ignore the model uncertainty of not knowing whether a VAR model is appropriate at all.
} 
length at $p_{\max }$ whereas a non-rejection starts a new testing procedure on the coefficients in front of the $\left(p_{\max }-1\right)$ 'th lag, etc. The estimated lag order is found as soon as the first rejection occurs. Alternatively, we can rely on residual based misspecification tests. This is intimately related to the sequential testing approach because we again systematically remove lags from the model until a rejection occurs ${ }^{3}$. The only difference is the type of test, namely a test on the residuals instead of a test on the nullity of a block of coefficients. More specifically, these tests check for the presence of serial correlation in the residuals. The rationale behind this approach is that not all the time dependency within the process can be accounted for when the lag order is underestimated. This dependency subsequently manifests itself in the form of correlated residuals. The detection of serial correlation is therefore a sign that the chosen autoregressive order is too small. Two timehonored references on univariate serial correlation tests are Breusch (1978) and Godfrey (1978). Their multivariate extensions are straightforward, see Chapter 4 of Lütkepohl (2005). Thirdly and finally, one may use information criteria to select the autoregressive order. These criteria balance the fit of the model against the dimension of the parameter space. Well-known examples are the Akaike Information Criteria (AIC, see Akaike (1998)), the Bayesian Information Criteria (BIC, see Schwarz (1978)) and the HannanQuinn Information Criteria (HQ, see Hannan and Quinn (1979) and Quinn (1980)). The sole difference between these criteria is the strength of the dimension penalty in terms of the number of parameters.

In Chapter 2, we study the properties of the Focused Information Criteria (FIC, see Claeskens and Hjort (2003)) for vector autoregressions. The main difference between this criteria and the AIC, BIC, and HQ is the explicit focus on a quantity of interest. We will mostly discuss impulse responses although the quantity of interest may take a much more general form. Additional, we apply the plug-in average by Liu (2015). Both the FIC and these plug-in averages are (and have been) motivated as minimizers of the asymptotic mean squared error within a setting containing local-to-zero parameters. We critically examine the implications of the inability to consistently estimate these local-tozero parameters.

Let us subsequently assume that we have selected the correct autoregressive order $p$. The functional form of the VAR is now fully specified. How should we estimate the parameters? One could specify a distribution on the innovations $\left\{\boldsymbol{u}_{t}\right\}$ and derive the implied

\footnotetext{
${ }^{3}$ Alternatively, it is also possible to start with a small number of lags and keep on adding lags until the test indicates that there is no longer any serial correlation in the residuals.
} 
maximum likelihood estimator (MLE). ${ }^{4}$ Alternatively, we could hope for a method that is fast to compute and allows for inference under fairly general assumptions. The least squares estimator has these characteristics and has therefore developed into the (almost) default estimation method. ${ }^{5}$ In Chapter 3 , we propose least absolute deviations (LAD) estimation as an alternative. This estimator is the maximum likelihood estimator under Laplacian innovations, it is computationally fast and similarly valid under a set of rather general conditions. We discuss the asymptotic distribution of the parameter estimates, tests on linear hypotheses (the Lagrange Multiplier, Likelihood Ratio and Wald test) and information criteria. As such, we attain a flexibility that equals the current least squares estimation. Having an alternative estimation method can be advantageous for two reasons. Firstly, our LAD estimator is shown to have lower mean squared errors and higher testing power if the innovation distribution has heavier tails. Secondly, there is now a fast alternative estimation method that allows results to be compared. This is surely relevant in view of model uncertainty. Two estimation methods that lead to similar conclusion should increase the analyst's confidence in the correctness of his/her results. On the contrary, different conclusion should incentivize the analyst to reconsider the model specification.

The applicability of bootstrap methods to LAD estimation of VARs is the topic of Chapter 4. In particular, we will proof the validity of the fixed design residual bootstrap. This method allows for asymptotically valid inference without density estimation. As such, it is a less involved approach because it is easier to re-estimate the parameters from pseudo-data than using density estimation.

\subsection{What not to expect from this thesis?}

Up to this point, I have mostly written about what you can find in this thesis. What will not be discussed? It is fruitful to spend some words on this question because its answer will highlight alternatives, shortcomings, and directions for further research. In particular, we will devote separate paragraphs to non-stationary VAR models and the Bayesian analysis of vector autoregressions.

We will focus exclusively on stable VAR models. This excludes cointegrating VARs. Cointegration occurs when several nonstationary variable combine linearly into a stationary relationship. The cointegration literature is rich and we do not intend to provide a full

\footnotetext{
${ }^{4}$ The MLE is often preferred because of its good performance (as quantified by the asymptotic variance). The MLE can also perform optimally, see for example chapter 33 of Cramér (1946) and Daniels (1961).

${ }^{5} \mathrm{~A}$ list of textbooks on vector autoregressions was provided on page 2 . All these textbook treat the least squares method in detail.
} 
literature survey. We simply mention two of the earlier reference, namely Granger (1981) and Engle and Granger (1987), as well as the textbooks by Johansen (1995) and Juselius (2006). However, we should emphasize that this literature relies strongly on least squares estimation. The references in the $\mathrm{LAD} /$ quantile literature that can be connected to cointegration are: (1) the paper by Koenker and Xiao (2004) on quantile unit root testing; and (2) the articles by Xiao (2009) and Cho et al. (2015) on the estimation of a single cointegrating relationship. In conclusion, results on $\mathrm{LAD} /$ quantile estimation of cointegrated VARs are currently scarse.

Throughout this thesis we will adopt the Frequentist approach. Frequentist inference starts from a statistical model in which parameters are fixed yet unknown quantities. Estimators are subsequently used to recover information about these true parameters. Bayesian inference treats the model parameters as random quantities. A prior distribution expresses the analyst's initial uncertainty about the parameters. The observed data in conjunction with the statistical model (more precisely the likelihood implied by the data) subsequently updates the analyst's beliefs to give the posterior distribution of the parameters.

The Bayesian approach offers two advantages. ${ }^{6}$ Firstly, the prior distribution can be used to shrink the richly parametrized ${ }^{7}$ VAR specification to parsimonious benchmarks. The earliest references in this respect is Litterman (1986) who chooses a prior specification that shrinks the VAR models towards univariate random walks with drift. We mention two extensions by Bańbura et al. (2010) and Giannone et al. (2015). Both articles consider large dimensional VARs. Bańbura et al. (2010) tighten the prior distributions around the parsimonious specification as more variables are included to handle the larger amount parameters. In this article, the hyperparameters governing the tightness of the prior distribution were selected ad hoc. Giannone et al. (2015) use hierarchical modeling to determine these hyperparameters and show that this increases forecasting performance and impulse

\footnotetext{
${ }^{6}$ I use the word 'advantages' but this might not be the correct word. It can give the impression that shrinkage and model averaging procedures cannot be implemented in the Frequentist approach. This is not true. The lasso, a shrinkage method, can be successfully applied to vector autoregressions (e.g. Kock and Callot (2015) and Kock (2016)). References on frequentist model averaging within the autoregressive context are Zhang et al. (2013) and Hansen (2016), as well as the plug-in estimator we propose in Chapter 2. However, on a personal note, I find that both shrinkage and model averaging are more natural within the Bayesian framework. They follow intuitively from the choice of the prior and by an additional application of Bayes' Theorem over a set of model priors, respectively. Within the Frequentist Approach one has to include a penalty term in the objective function (for shrinkage) and define explicitly how the model weights are determined (for model averaging).

${ }^{7}$ Each series is affected by $p$ lags of the $K$ variables in the system so this leads to $K p$ parameters per series. Since there are $K$ series, this makes $K^{2} p$ autoregressive coefficients in total. Additionally, the $K(K+1) / 2$ parameters specifying the covariance matrix of $\boldsymbol{u}_{t}=\left(u_{1, t}, \ldots, u_{K, t}\right)^{\prime}$ might be relevant for the analysis (e.g. for the estimation of impulse responses). The number of parameters will be even larger if deterministic terms are included. In general, the number of parameters grows fast with increasing $K$.
} 
response estimation accuracy. Secondly, model uncertainty can be easily accounted for by Bayesian model averaging. The articles by Strachan and van Dijk (2007) and Garratt et al. (2009) are illustrative since these authors consider a variety ${ }^{8}$ of VAR specifications. The resulting posterior distributions are weighted averages across the models. These weights reflect the credibility of the individual models.

\subsection{Outline}

The content of the Chapters 2-4 has been introduced in the previous pages. Additionally, you can also find an abstract at the start of every chapter to give further details on their content. A quick summary is as follows: Chapter 2 introduces a Focused Information Criterion to take into account the uncertainty of the lag order of the VAR; in Chapter 3, we consider parameter estimation, hypothesis testing and information criteria for VAR models estimated by least absolute deviations; bootstrap approaches to LAD estimation are covered in Chapter 4. In the concluding chapter, Chapter 5, we will end this thesis by restarting from the beginning. That is, we will review both the introduction and the core chapters. The conclusion will also contain a short outlook on further research questions. The bibliography, valorisation, Dutch summary and my curriculum vitae are included as final attachments.

\subsection{Notation}

Some short words on notation. We will try to follow the notational conventions by Abadir and Magnus (2002) as closely as possible. In particular, $\stackrel{d}{\longrightarrow}$ and $\stackrel{p}{\longrightarrow}$ signify convergence in distribution and convergence in probability, respectively. The stochastic and the strict stochastic order relations are denoted by $O_{p}(\cdot)$ and $o_{p}(\cdot)$. Vectors are printed in bold and denote column vectors by default. ${ }^{9} \mathbf{0}_{j}$ is the column vector of length $j$ consisting of zeros only. For an $n$-vector $\boldsymbol{a}=\left(a_{1}, a_{2}, \cdots, a_{n}\right)^{\prime}$ we have the usual $p$-norm $\|\boldsymbol{a}\|_{p}=$ $\left(\sum_{i=1}^{n}\left|a_{i}\right|^{p}\right)^{1 / p}$. Matrix norms are taken entry-wise. That is, for any matrix $\boldsymbol{A}$ its $p$-norm, $\|\boldsymbol{A}\|_{p}$, is given by $\|\boldsymbol{A}\|_{p}=\|\operatorname{vec}(\boldsymbol{A})\|_{p}$. The subscript $p$ in the norms is omitted if $p=2$. The sign function $\operatorname{sgn}(\cdot)$ works on the individual elements of a vector. Further notational conventions are made explicit at the specific place of interest.

\footnotetext{
${ }^{8}$ In Strachan and van Dijk (2007), these different VAR specification express the model uncertainty related to the number of cointegrating relations and the deterministic components to be included in the model.

${ }^{9} \mathrm{We}$ will allow for some notational freedom in Chapter 2 to keep several definitions in line with the earlier results by Liu (2015). Also in Chapter 2, we define the Jacobian matrices $\boldsymbol{D}_{\theta}=\partial \boldsymbol{\mu}\left(\boldsymbol{\theta}_{\infty}, \boldsymbol{\sigma}\right) / \partial \boldsymbol{\theta}^{\prime}$ and $\boldsymbol{D}_{\sigma}=$ $\partial \boldsymbol{\mu}\left(\boldsymbol{\theta}_{\infty}, \boldsymbol{\sigma}\right) / \partial \boldsymbol{\sigma}^{\prime}$. Note that these matrices turn into row vectors when the function $\mu$ is scalar.
} 


\section{Chapter 2}

\section{A Focused Information Criterion for Locally Misspecified Vector Autoregressive Models}

"Science is about finding ever better approximations rather than pretending you have already found the ultimate truth. A casual stroll through the lunatic asylum shows that faith does not prove anything." - Friedrich Nietzsche (1844-1900) 


\section{Abstract $^{\dagger}$}

This chapter investigates the Focused Information Criterion (abbreviated FIC, Claeskens and Hjort (2003)) in autoregressive models with local-to-zero misspecification. The main advantage of the FIC is that model selection/averaging are focused on the quantity of interest instead of aiming at some measure of global model fit. The quantity of interest is allowed to be any (sufficiently regular) function of the parameters. Asymptotic properties are derived and we elaborate on the role of the locally misspecified parameters. These parameters cannot be estimated consistently and we show that this translates into suboptimal selection and averaging procedures. Inspired by the work of Hansen (2005), we apply our estimation method to impulse responses. Monte Carlo simulations show that the FIC does not perform as well as the infeasible criteria that are based on the true locally misspecified parameters. The feasible FIC performs similarly to AIC (for model selection) and smoothed AIC and Jackknife Model Averaging (for model averaging) at small amounts of misspecification but outperforms its feasible competitors when the misspecification becomes larger.

\footnotetext{
${ }^{\dagger}$ This chapter is based on joint work with J. Lohmeyer, F.C. Palm and J.-P. Urbain. It is forthcoming in Econometric Reviews.
} 


\subsection{Introduction}

The motivation for this chapter stems from Hansen (2005). The author considers a Gaussian $\operatorname{ARMA}(1,1)$ model approximated by $\operatorname{AR}(k)$ models with $k \in\left\{0,1, \cdots, k_{\text {max }}\right\}$ and is interested in the impulse responses. Table 1 of Hansen (2005) shows that the MSEminimizing AR order depends strongly on parameter values and impulse response horizon. An extreme case is the specification $y_{t}=0.5 y_{t-1}+\epsilon_{t}-0.9 \epsilon_{t-1}$. The MSE-minimizing autoregressive orders equal 0 and 10 for the impulse responses at horizon 2 and 6 , respectively. Ivanov and Kilian (2005) report a similar issue in a VAR setting. They simulate VAR processes similar to those often found in empirical work, and rank different model selection criteria (AIC, BIC, HQ and serial correlation tests) based on the MSE of the estimated impulse responses implied by the selected model. A uniformly best criterion was not found. This might be expected since information criteria like AIC and BIC aim at global model fit and do not take into account the quantity of interest (such as the impulse response at a particular horizon). The Focused Information Criteria introduced by Claeskens and Hjort (2003) takes into account the interest of the researcher. Hansen (2005) acknowledged the opportunities for the FIC for the estimation of impulse responses when he remarked based on simulation outcomes: "The message from Tables 2 and 3 is that the FIC is an intriguing challenger to existing model selection methods and deserves attention and scrutiny". A theoretical justification of his simulation results was not provided.

We develop a theoretical framework starting from a vector autoregression where part of the coefficients are local-to-zero, i.e. declining to zero at a rate of $T^{-1 / 2}$ with $T$ denoting sample size. This case is fundamentally different from a static setup because dynamic properties are also varying with sample size. ${ }^{1}$ Building on ideas from Claeskens and Hjort (2003), Claeskens and Hjort (2008) and Liu (2015), we propose an estimator that can be used for both model selection and model averaging. This estimator is fairly general as it only requires the parameters of interest to be a sufficiently smooth transformation of the model's parameters. The results are subsequently applied to the specific case of impulse responses. A slight generalization of a theorem by Liu (2015) enables us to not only construct confidence intervals for a specific horizon, but to also construct confidence bands for multiple horizons. Additionally, we provide an in depth discussion on the role

\footnotetext{
${ }^{1}$ Dynamic models under local-to-zero misspecification were discussed in Claeskens et al. (2007) and Rohan and Ramanathan (2011). Both papers first derive the asymptotic results in a setting without local misspecification and subsequently introduce the misspecification (see p. 363 of Claeskens et al. (2007) and Equation (8) on p. 221 of Rohan and Ramanathan (2011)). The theoretical implications of this two step procedure are not completely clear.
} 
of the local-to-zero parameters. These parameters cannot be estimated consistently, and we show that as a consequence the FIC and plug-in averages do not truly minimize the asymptotic mean squared error.

Our paper is related to the literature on model selection/averaging and the literature on impulse response analysis. We now discuss both. One of the earliest references on frequentist model averaging is the paper by Bates and Granger (1969). The literature on model averaging that is unrelated to forecasting is of a more recent origin. One literature branch on frequentist model averaging started with the paper by Hansen (2007). This paper shows that weight selection by minimization of Mallow's criterion will asymptotically lead to the lowest possible squared error among a class of estimators. His regression setup with homoskedastic errors was generalized to regression forecasts in Hansen (2008), and was modified by Hansen and Racine (2012) to allow for heteroskedastic errors. A time series application to stationary autoregressions of infinite order is Zhang et al. (2013). Zhang and Liu (2017) report results on the distribution of Mallow's and Jackknife-based model averaging weights in linear regressions with irrelevant variables.

The second branch of literature on frequentist model averaging evolves around locally misspecified models. The FIC was proposed by Claeskens and Hjort (2003) and extended by Claeskens and Hjort (2008). The underlying idea has been applied to various settings. We will report a non-exhaustive list. Liu (2015) considered the linear regression setup and derived asymptotically valid confidence intervals. Two additions to the treatment effects literature are Lu (2015) and Kitagawa and Muris (2016). DiTraglia (2016) provides results for generalized method of moments estimation. Liu and Kuo (2016) consider predictive regressions.

Finally, we briefly discuss the literature on impulse response (IR) analysis in autoregressive models. A comprehensive discussion on IR analysis can be found in section 3.7 of Lütkepohl (2005). Both Lütkepohl (1990) and Benkwitz et al. (2000) have reported that the coverage of the impulse response confidence intervals can be low since the convergence rate of the estimators to their asymptotic distribution is non-constant over the complete parameter space. Another important topic for impulse response analysis is the construction of joint confidence bands. A naive Cartesian product of the individual confidence intervals leads to severe undercoverage, whereas confidence bands based on the Bonferroni inequality have good coverage but are at the same time excessively wide. Often considered alternatives are based on bootstrap methods, e.g. Kilian (2001), Lütkepohl et al. (2015) and Bruder and Wolf (2017). 
This chapter is organized as follows. Section 2.2 presents the model framework, the estimation procedure, and the asymptotic properties of: (1) the parameter estimates, (2) the feasible FIC, and (3) the elements of the weighting matrix. A discussion and illustration of the consequences of the inconsistent estimation of the local-to-zero parameter follows. Our theoretical findings are subsequently supported by various Monte Carlo simulations in Section 2.3. Section 2.4 concludes, and the mathematical proofs are presented in the appendix of this chapter.

\subsection{Theory}

\subsubsection{The Model Framework}

Let the $K$-dimensional multiple time series $\left\{\left\{\boldsymbol{y}_{T, t}\right\}_{t=-\infty}^{\infty}\right\}_{T=1}^{\infty}$ constitute a vector triangular array generated by the vector autoregressive (VAR) processes

$$
\boldsymbol{y}_{T, t}=\boldsymbol{B}_{1} \boldsymbol{y}_{T, t-1}+\ldots+\boldsymbol{B}_{p_{1}} \boldsymbol{y}_{T, t-p_{1}}+\frac{\boldsymbol{\Delta}_{1}}{\sqrt{T}} \boldsymbol{y}_{T, t-p_{1}-1}+\ldots+\frac{\boldsymbol{\Delta}_{p_{2}}}{\sqrt{T}} \boldsymbol{y}_{T, t-p_{1}-p_{2}}+\boldsymbol{u}_{t}
$$

where $\boldsymbol{B}_{i}\left(i \in\left\{1,2, \ldots, p_{1}\right\}\right)$ and $\boldsymbol{\Delta}_{i}\left(i \in\left\{1,2, \ldots, p_{2}\right\}\right)$ are $(K \times K)$ coefficient matrices. Equation (2.1) differs in one important aspect from the usual VAR specifications, namely some of the coefficient matrices are premultiplied by $T^{-1 / 2}$ with $T$ denoting sample size. This local-to-zero misspecification causes different dynamics for every $T$. Mathematically, this decay rate will prove to be crucial for the development of the asymptotic theory because it prevents the omitted variable bias from diverging with increasing sample size. Intuitively, we could think of this model specification as expressing a degree of uncertainty concerning the true lag order. The VAR process includes $p:=p_{1}+p_{2}$ lags for finite $T$, yet asymptotically a $\operatorname{VAR}\left(p_{1}\right)$ remains. This can be interpreted as exploring a shrinking neighborhood of the $\operatorname{VAR}\left(p_{1}\right)$ model. The locally misspecified VAR model of Equation (2.1) is thus used as an approximation to processes that behave similarly to a stationary $\operatorname{VAR}\left(p_{1}\right)$.

\subsubsection{Parameter Estimation and Asymptotics}

To simplify notation, we collect all the parameters in the matrices $\boldsymbol{B}=\left[\begin{array}{llll}\boldsymbol{B}_{1} & \boldsymbol{B}_{2} & \cdots & \boldsymbol{B}_{p_{1}}\end{array}\right]$, $\boldsymbol{C}_{T}=\left[\begin{array}{llll}\boldsymbol{\Delta}_{1} & \boldsymbol{\Delta}_{2} & \cdots & \boldsymbol{\Delta}_{p_{2}}\end{array}\right] / \sqrt{T}=\boldsymbol{\Delta} / \sqrt{T}$, and define $\boldsymbol{\Theta}_{T}=\left[\begin{array}{ll}\boldsymbol{B} \boldsymbol{C}_{T}\end{array}\right]$. Similarly to 
Lütkepohl (2005), we also stack the observations over time to obtain,

$$
\begin{aligned}
& \boldsymbol{Y}_{T}:=\left(\boldsymbol{y}_{T, 1}, \boldsymbol{y}_{T, 2}, \ldots, \boldsymbol{y}_{T, T}\right) \quad(K \times T), \\
& \boldsymbol{z}_{T, t}:=\left[\begin{array}{c}
\boldsymbol{y}_{T, t} \\
\boldsymbol{y}_{T, t-1} \\
\vdots \\
\boldsymbol{y}_{T, t-p+1}
\end{array}\right] \quad(K p \times 1), \\
& \boldsymbol{Z}_{T}:=\left(\boldsymbol{z}_{T, 0}, \boldsymbol{z}_{T, 1}, \ldots, \boldsymbol{z}_{T, T-1}\right) \quad(K p \times T), \\
& \boldsymbol{U}:=\left(\boldsymbol{u}_{1}, \boldsymbol{u}_{2}, \ldots, \boldsymbol{u}_{T}\right) \quad(K \times T) .
\end{aligned}
$$

The model can now be expressed as $\boldsymbol{Y}_{T}=\boldsymbol{\Theta}_{T} \boldsymbol{Z}_{T}+\boldsymbol{U}$. A variety of approximating models can be considered but we will restrict our attention to models that use the same lag order in every equation (see Remark 2.1 for further details). Using the same lag order in all crosssections is common practice and will decrease the notational burden. Selection matrices are used to relate all estimators to the estimator using $p$ lags. That is, for some integer $m$ such that $p_{1} \leq m \leq p$,

$$
\begin{array}{rlr}
\boldsymbol{L}:=\boldsymbol{L}^{(1)} \otimes \boldsymbol{I}_{K}, \text { with } \boldsymbol{L}^{(1)}=\left[\begin{array}{c}
\boldsymbol{I}_{p_{1}} \\
\mathbf{O}_{p_{2} \times p_{1}}
\end{array}\right] & \left(K p \times K p_{1}\right), \\
\boldsymbol{S}_{0}:=\boldsymbol{S}_{0}^{(1)} \otimes \boldsymbol{I}_{K}, \text { with } \boldsymbol{S}_{0}^{(1)}=\left[\begin{array}{c}
\mathbf{O}_{p_{1} \times p_{2}} \\
\boldsymbol{I}_{p_{2}}
\end{array}\right] & \left(K p \times K p_{2}\right), \\
\boldsymbol{S}_{m}:=\boldsymbol{S}_{m}^{(1)} \otimes \boldsymbol{I}_{K}, \text { with } \boldsymbol{S}_{m}^{(1)}=\left[\begin{array}{c}
\boldsymbol{I}_{m} \\
\mathbf{O}_{(p-m) \times m}
\end{array}\right] & (K p \times K m), \\
\boldsymbol{\Pi}_{m}^{\prime}:=\boldsymbol{\Pi}_{m}^{(1)} \otimes \boldsymbol{I}_{K}, \text { with } \boldsymbol{\Pi}_{m}^{\prime(1)}=\left[\begin{array}{c}
\boldsymbol{I}_{m-p_{1}} \\
\mathbf{O}_{(p-m) \times\left(m-p_{1}\right)}
\end{array}\right] & \left(K p_{2} \times K\left(m-p_{1}\right)\right) .
\end{array}
$$

The Kronecker products with $\boldsymbol{I}_{K}$ are a direct consequence of estimating all equations with the same lag order. The regressor matrix for the estimation of a $\operatorname{VAR}(m)$ model satisfies $\boldsymbol{Z}_{T, m}=\boldsymbol{S}_{m}^{\prime} \boldsymbol{Z}_{T}$. The implied OLS estimator is the $(K \times K m)$ matrix $\hat{\boldsymbol{\Theta}}_{T, m}$ given by

$$
\hat{\boldsymbol{\Theta}}_{T, m}=\boldsymbol{\Theta}_{T, m}+\boldsymbol{C}_{T}\left(\boldsymbol{I}_{K p_{2}}-\boldsymbol{\Pi}_{m}^{\prime} \boldsymbol{\Pi}_{m}\right) \boldsymbol{S}_{0}^{\prime} \boldsymbol{Z}_{T} \boldsymbol{Z}_{T, m}^{\prime}\left(\boldsymbol{Z}_{T, m} \boldsymbol{Z}_{T, m}^{\prime}\right)^{-1}+\boldsymbol{U} \boldsymbol{Z}_{T, m}^{\prime}\left(\boldsymbol{Z}_{T, m} \boldsymbol{Z}_{T, m}^{\prime}\right)^{-1}
$$


Some rearranging and rescaling produces,

$$
\begin{gathered}
\sqrt{T}\left(\hat{\Theta}_{T, m}-\Theta_{T, m}\right)=\underbrace{\sqrt{T} \boldsymbol{C}_{T}}_{\boldsymbol{\Delta}}\left(\boldsymbol{I}_{K p_{2}}-\boldsymbol{\Pi}_{m}^{\prime} \boldsymbol{\Pi}_{m}\right) \boldsymbol{S}_{0}^{\prime}\left(\frac{1}{T} \boldsymbol{Z}_{T} \boldsymbol{Z}_{T}^{\prime}\right) \boldsymbol{S}_{m}\left[\boldsymbol{S}_{m}^{\prime}\left(\frac{1}{T} \boldsymbol{Z}_{T} \boldsymbol{Z}_{T}^{\prime}\right) \boldsymbol{S}_{m}\right]^{-1} \\
+\left(\frac{1}{\sqrt{T}} \boldsymbol{U} \boldsymbol{Z}_{T}^{\prime}\right) \boldsymbol{S}_{m}\left[\boldsymbol{S}_{m}^{\prime}\left(\frac{1}{T} \boldsymbol{Z}_{T} \boldsymbol{Z}_{T}^{\prime}\right) \boldsymbol{S}_{m}\right]^{-1}
\end{gathered}
$$

and it can be seen that the $T^{1 / 2}$-consistency of the estimator precisely matches the decay rate of $T^{-1 / 2}$ in the elements of the parameter matrix $\boldsymbol{C}_{T}$. As a final step we apply the vectorization operator to transform the parameter matrices into a single parameter vector,

$$
\begin{aligned}
\sqrt{T}\left(\hat{\boldsymbol{\theta}}_{T, m}-\boldsymbol{\theta}_{T, m}\right)=( & {\left.\left[\boldsymbol{S}_{m}^{\prime}\left(\frac{1}{T} \boldsymbol{Z}_{T} \boldsymbol{Z}_{T}^{\prime}\right) \boldsymbol{S}_{m}\right]^{-1} \boldsymbol{S}_{m}^{\prime}\left(\frac{1}{T} \boldsymbol{Z}_{T} \boldsymbol{Z}_{T}^{\prime}\right) \boldsymbol{S}_{0}\left(\boldsymbol{I}_{K p_{2}}-\boldsymbol{\Pi}_{m}^{\prime} \boldsymbol{\Pi}_{m}\right) \otimes \boldsymbol{I}_{K}\right) \boldsymbol{\delta} } \\
& +\left(\left[\boldsymbol{S}_{m}^{\prime}\left(\frac{1}{T} \boldsymbol{Z}_{T} \boldsymbol{Z}_{T}^{\prime}\right) \boldsymbol{S}_{m}\right]^{-1} \boldsymbol{S}_{m}^{\prime} \otimes \boldsymbol{I}_{K}\right) \frac{1}{\sqrt{T}} \sum_{t=1}^{T} \operatorname{vec}\left(\boldsymbol{u}_{t} \boldsymbol{z}_{T, t-1}^{\prime}\right),
\end{aligned}
$$

where $\hat{\boldsymbol{\theta}}_{T, m}=\operatorname{vec}\left(\hat{\boldsymbol{\Theta}}_{T, m}\right), \boldsymbol{\theta}_{T, m}=\operatorname{vec}\left(\boldsymbol{\Theta}_{T, m}\right)$, and $\boldsymbol{\delta}=\operatorname{vec}(\boldsymbol{\Delta})$. Equation (2.6) depends on: (1) various selection matrices, (2) the random matrix $\frac{1}{T} \boldsymbol{Z}_{T} \boldsymbol{Z}_{T}^{\prime}=\frac{1}{T} \sum_{t=1}^{T} \boldsymbol{z}_{T, t-1} \boldsymbol{z}_{T, t-1}^{\prime}$, and (3) the random vector $\frac{1}{\sqrt{T}} \sum_{t=1}^{T} \operatorname{vec}\left(\boldsymbol{u}_{t} \boldsymbol{z}_{T, t-1}^{\prime}\right)$. The latter two rescaled sums are typically encountered in laws of large numbers and central limit theorems, respectively. The following three assumptions guarantee that such theorems are applicable.

Assumption 1 The sequence $\left\{\boldsymbol{u}_{t}\right\}$ of random $K$-vectors is an independent and identically distributed sequence with mean vector zero, a positive definite covariance matrix $\mathrm{E}\left(\boldsymbol{u}_{t} \boldsymbol{u}_{t}^{\prime}\right)=\Sigma$, and there exists a $c>0$ such that $\mathrm{E}\left|u_{i t} u_{j t} u_{k t} u_{m t}\right|<c<\infty$ for all $i, j, k, m=1,2, \ldots K$.

Assumption $2 \operatorname{det}\left(\boldsymbol{B}_{T}(z)\right)=\operatorname{det}\left(\boldsymbol{I}_{K} z^{p}-\boldsymbol{B}_{1} z^{p-1}-\ldots-\boldsymbol{B}_{p_{1}} z^{p_{2}}-\frac{\boldsymbol{\Delta}_{1}}{\sqrt{T}} z^{p_{2}-1}-\ldots-\frac{\boldsymbol{\Delta}_{p_{2}}}{\sqrt{T}}\right) \neq$ 0 for all $|z| \geq 1$ and $\forall T \in \mathbb{N}$.

Assumption $3 \operatorname{det}\left(\boldsymbol{B}_{\infty}(z)\right)=\operatorname{det}\left(\boldsymbol{I}_{K} z^{p_{1}}-\boldsymbol{B}_{1} z^{p_{1}-1}-\ldots-\boldsymbol{B}_{p_{1}}\right) \neq 0$ for all $|z| \geq 1$ 
Assumption 1 provides moment bounds and independence between the innovation $\boldsymbol{u}_{t}$ and its past. This latter property is exploited to apply limit theorems for martingale differences. $^{2}$ Assumptions 2 and 3 require the vector autoregressive process to be stationary for every finite $T$ and also in the absence of local misspecification. The asymptotic properties of the OLS estimators are stated in Theorem 1.

\section{Theorem 1: Asymptotic Normality of the Least Squares Estimator}

\section{Let Assumptions 1-3 hold. Then}

(a) In the limit $T \rightarrow \infty$, we have for any $m \in \mathcal{M}=\left\{p_{1}, p_{1}+1, \ldots, p\right\}$,

$$
\sqrt{T}\left(\hat{\boldsymbol{\theta}}_{T, m}-\boldsymbol{\theta}_{T, m}\right) \stackrel{d}{\longrightarrow} \boldsymbol{A}_{m} \boldsymbol{\delta}+\left(\left[\boldsymbol{S}_{m}^{\prime} \boldsymbol{\Omega} \boldsymbol{S}_{m}\right]^{-1} \boldsymbol{S}_{m}^{\prime} \otimes \boldsymbol{I}_{K}\right) \boldsymbol{R} \sim \mathrm{N}\left(\boldsymbol{A}_{m} \boldsymbol{\delta},\left[\boldsymbol{S}_{m}^{\prime} \boldsymbol{\Omega} \boldsymbol{S}_{m}\right]^{-1} \otimes \boldsymbol{\Sigma}\right),
$$

with $\boldsymbol{\Omega}=\operatorname{plim}_{T \rightarrow \infty} \frac{1}{T} \sum_{t=1}^{T} \boldsymbol{z}_{T, t-1} \boldsymbol{z}_{T, t-1}^{\prime}, \boldsymbol{A}_{m}=\left[\boldsymbol{S}_{m}^{\prime} \boldsymbol{\Omega} \boldsymbol{S}_{m}\right]^{-1} \boldsymbol{S}_{m}^{\prime} \boldsymbol{\Omega} \boldsymbol{S}_{0}\left(\boldsymbol{I}_{K p_{2}}-\boldsymbol{\Pi}_{m}^{\prime} \boldsymbol{\Pi}_{m}\right) \otimes$ $\boldsymbol{I}_{K}$ and $\boldsymbol{R} \sim \mathrm{N}(\mathbf{0}, \boldsymbol{\Omega} \otimes \boldsymbol{\Sigma})$.

(b) Let $\hat{\boldsymbol{u}}_{T, t}^{m}$ denote the OLS residuals from the estimation of a VAR(m). Consider $\hat{\boldsymbol{\Sigma}}_{u}^{m}=$ $\frac{1}{T} \sum_{t=1}^{T} \hat{\boldsymbol{u}}_{T, t}^{m} \hat{\boldsymbol{u}}_{T, t}^{m \prime}$ as an estimator for $\boldsymbol{\Sigma}_{u}$. The result in part (a) can be strengthened to joint asymptotic normality with the covariance matrix estimator $\hat{\boldsymbol{\Sigma}}_{u}^{m}$,

$$
\left[\begin{array}{c}
\sqrt{T}\left(\hat{\boldsymbol{\theta}}_{T, m}-\boldsymbol{\theta}_{T, m}\right) \\
\sqrt{T} \operatorname{vech}\left(\hat{\Sigma}_{u}^{m}-\boldsymbol{\Sigma}\right)
\end{array}\right] \stackrel{d}{\longrightarrow} \mathrm{N}\left(\left[\begin{array}{c}
\boldsymbol{A}_{m} \\
\mathbf{O}
\end{array}\right] \boldsymbol{\delta},\left[\begin{array}{cc}
{\left[\boldsymbol{S}_{m}^{\prime} \boldsymbol{\Omega} \boldsymbol{S}_{m}\right]^{-1} \otimes \boldsymbol{\Sigma}} & \mathbf{O} \\
\mathbf{O} & \boldsymbol{\Xi}_{22}
\end{array}\right]\right)
$$

The matrix $\boldsymbol{\Xi}_{22}$ is specified in the Appendix.

(c) The estimator convergence as discussed in parts (a) and (b) of this theorem is also a joint convergence across different $m \in \mathcal{M}$. That is, for $\left\{i_{1}, i_{2}, \ldots, i_{M}\right\} \in \mathcal{M}$, any $m \in \mathcal{M}$, and $i_{1}<i_{2}<\ldots<i_{M}$, we have

$$
\left[\begin{array}{c}
\sqrt{T}\left(\hat{\boldsymbol{\theta}}_{T, i_{1}}-\boldsymbol{\theta}_{i_{1}}\right) \\
\sqrt{T}\left(\hat{\boldsymbol{\theta}}_{T, i_{2}}-\boldsymbol{\theta}_{i_{2}}\right) \\
\vdots \\
\sqrt{T}\left(\hat{\boldsymbol{\theta}}_{T, i_{M}}-\boldsymbol{\theta}_{i_{M}}\right) \\
\sqrt{T} \operatorname{vech}\left(\hat{\boldsymbol{\Sigma}}_{u}^{m}-\boldsymbol{\Sigma}\right)
\end{array}\right] \stackrel{d}{\longrightarrow} \mathrm{N}\left(\left[\begin{array}{c}
\boldsymbol{A}_{i_{1}} \\
\boldsymbol{A}_{i_{2}} \\
\vdots \\
\boldsymbol{A}_{i_{M}} \\
\mathbf{O}
\end{array}\right] \boldsymbol{\delta},\left[\begin{array}{ccccc}
\boldsymbol{V}_{i_{1} i_{1}} & \boldsymbol{V}_{i_{1} i_{2}} & \ldots & \boldsymbol{V}_{i_{1} i_{M}} & \mathbf{0} \\
\boldsymbol{V}_{i_{2} i_{1}} & \boldsymbol{V}_{i_{2} i_{2}} & \ldots & \boldsymbol{V}_{i_{2} i_{M}} & \mathbf{O} \\
\vdots & \vdots & \ddots & \vdots & \vdots \\
\boldsymbol{V}_{i_{M} i_{1}} & \boldsymbol{V}_{i_{M} i_{2}} & \ldots & \boldsymbol{V}_{i_{M} i_{M}} & \mathbf{O} \\
\mathbf{O} & \mathbf{O} & \ldots & \mathbf{O} & \boldsymbol{\Xi}_{22}
\end{array}\right]\right) .
$$

\footnotetext{
${ }^{2}$ The requirement of i.i.d. innovations can be relaxed to the assumption that $\left\{\boldsymbol{u}_{t}\right\}$ is a martingale difference sequence. Formally, let $\mathcal{F}_{t}=\sigma\left(\boldsymbol{u}_{s},-\infty<s \leq t\right)$ denote the sigma field generated by the innovations up to and including time $t$. Our results remain valid if the conditions $\mathrm{E}\left(\boldsymbol{u}_{t}\right)=\mathbf{0}$ and $\mathrm{E}\left(\boldsymbol{u}_{t} \boldsymbol{u}_{t}^{\prime}\right)=\boldsymbol{\Sigma}$ are replaced by $\mathrm{E}\left(\boldsymbol{u}_{t} \mid \mathcal{F}_{t-1}\right)=\mathbf{0}$ and $\mathrm{E}\left(\boldsymbol{u}_{t} \boldsymbol{u}_{t}^{\prime} \mid \mathcal{F}_{t-1}\right)=\boldsymbol{\Sigma}$, respectively.
} 
The matrices $\boldsymbol{V}_{j k}$ are given by $\boldsymbol{V}_{j k}=\left[\boldsymbol{S}_{j}^{\prime} \boldsymbol{\Omega} \boldsymbol{S}_{j}\right]^{-1} \boldsymbol{S}_{j}^{\prime} \boldsymbol{\Omega} \boldsymbol{S}_{k}\left[\boldsymbol{S}_{k}^{\prime} \boldsymbol{\Omega} \boldsymbol{S}_{k}\right]^{-1} \otimes \boldsymbol{\Sigma}$. It suffices to consider a single estimator for $\Sigma$ because all the estimators are asymptotically equivalent.

The matrix $\Omega$ deserves further attention. It is defined as the probability limit of the Gram matrix $\frac{1}{T} \boldsymbol{Z}_{T} \boldsymbol{Z}_{T}^{\prime}$. The proof of Theorem 1 reveals that this probability limit equals $\mathrm{E}\left(\boldsymbol{z}_{\infty, t} \boldsymbol{z}_{\infty, t}^{\prime}\right)$ where $\boldsymbol{z}_{\infty, t}$ is defined as in Equation (2.2) but being generated by a VAR without local misspecification. We illustrate this remark with the AR process defined by $y_{T, t}=\alpha y_{T, t-1}+\frac{\delta_{1}}{\sqrt{T}} y_{T, t-2}+\frac{\delta_{2}}{\sqrt{T}} y_{T, t-3}+u_{t}$, that is an AR model with $p_{1}=1, p_{2}=2$ and $p=3$. We would find $\Omega=\frac{\sigma^{2}}{1-\alpha^{2}}\left[\begin{array}{ccc}1 & \alpha & \alpha^{2} \\ \alpha & 1 & \alpha \\ \alpha^{2} & \alpha & 1\end{array}\right]$.

Remark 2.1 The consequences of the local misspecification framework are visible in Theorem 1. Standard asymptotics will fail if relevant parameters are left out since omitted variable bias will dominate asymptotically. ${ }^{3}$ The local-to-zero rate of $T^{-1 / 2}$ balances this diverging behavior such that a finite asymptotic bias remains. This reasoning applies to all models that contain all the fixed parameters (i.e. the lag order should be no less than $p_{1}$ ) and leave out arbitrary parameters that are local-to-zero.

Remark 2.2 Assumption 2 is rather strict because it requires stationarity for all $T$ in the natural numbers. Is it even possible for any parameter combination to satisfy this assumption? We can answer this question in the affirmative for the univariate case but the result does not generalize easily to the multivariate case. For the univariate case, we define the lag polynomial $\beta_{T}(L)$ by

$$
\beta_{T}(L) y_{T, t}=\left(1-\beta_{1} L-\ldots-\beta_{p_{1}} L^{p_{1}}-\frac{\delta_{1}}{\sqrt{T}} L^{p_{1}+1}-\ldots-\frac{\delta_{p_{2}}}{\sqrt{T}} L^{p}\right) y_{T, t}=u_{t} .
$$

Fujiwara (1916) has shown that the largest modulus root of a polynomial $a(z)=a_{0} z^{n}+$ $a_{1} z^{n-1}+\ldots+a_{n-1} z+a_{n}$ is bounded above by $2 \max \left\{\left|a_{1} / a_{0}\right|,\left|a_{2} / a_{0}\right|^{1 / 2}, \ldots,\left|a_{n} / a_{0}\right|^{1 / n}\right\}$. The largest modulus root of the lag polynomial $\beta_{T}$ is thus bounded by

$$
2 \max \left\{\left|\beta_{1}\right|,\left|\beta_{2}\right|^{\frac{1}{2}}, \ldots,\left|\beta_{p_{1}}\right|^{\frac{1}{p_{1}}},\left|\frac{\delta_{1}}{\sqrt{T}}\right|^{\frac{1}{p_{1}+1}}, \ldots,\left|\frac{\delta_{p_{2}}}{\sqrt{T}}\right|^{\frac{1}{p}}\right\}
$$

\footnotetext{
${ }^{3}$ Let us consider the data generating process $y_{t}=\alpha_{1} y_{t-1}+\alpha_{2} y_{t-2}+u_{t}$. Suppose that we estimate an AR(1) model. The OLS parameter estimator of the first lag coefficient, say $\hat{\alpha}_{1}$, satisfies $\sqrt{T}\left(\hat{\alpha}_{1}-\alpha_{1}\right)=$ $\sqrt{T} \alpha_{2} \frac{\frac{1}{T} \sum_{t=1}^{T} y_{t-1} y_{t-2}}{\frac{1}{T} \sum_{t=1}^{T} y_{t-1}^{2}}+\frac{\frac{1}{\sqrt{T}} \sum_{t=1}^{T} y_{t-1} u_{t}}{\frac{1}{T} \sum_{t=1}^{T} y_{t-1}^{2}}$. The first term on the RHS diverges for large $T$ if $\alpha_{2} \neq 0$. The divergence rate is $\sqrt{T}$.
} 
We deduce from Equation (2.8) that $2 \max \left\{\left|\beta_{1}\right|,\left|\beta_{2}\right|^{\frac{1}{2}}, \ldots,\left|\beta_{p_{1}}\right|^{\frac{1}{p_{1}}},\left|\delta_{1}\right|^{\frac{1}{p_{1}+1}}, \ldots,\left|\delta_{p_{2}}\right|^{\frac{1}{p}}\right\}<1$ guarantees stationarity for all $T^{4}$

\subsubsection{The Quantities of Interest}

The Focused Information Criterion (FIC) introduced by Claeskens and Hjort (2003) focusses on a quantity of interest rather than general model fit. Quantities of interest could be a single parameter, several parameters, or parameter transformations. Natural quantities of interest in the current dynamical setting are the impulse responses. In general, let $\boldsymbol{\mu}: \mathbb{R}^{K^{2} p+K(K+1) / 2} \rightarrow \mathbb{R}^{l}$ define the mapping from the model parameters to the $l$ dimensional focus quantity. The first $K^{2} p$ arguments of the function $\boldsymbol{\mu}$ are reserved for the conditional mean parameters, whereas the last $K(K+1) / 2$ arguments refer to the parameters in $\boldsymbol{\Sigma}$. As such we define $\boldsymbol{\sigma}=\operatorname{vech}(\boldsymbol{\Sigma})$ and $\hat{\boldsymbol{\sigma}}=\operatorname{vech}(\hat{\boldsymbol{\Sigma}})$, and write $\mu(\boldsymbol{\theta}, \boldsymbol{\sigma}){ }^{5}$ We additionally assume that evaluating the quantity of interest at $\boldsymbol{\mu}\left(\left(\boldsymbol{\theta}_{T, m}, \mathbf{0}_{K^{2}(p-m)}\right), \boldsymbol{\sigma}\right)$ provides an estimate for the quantity of interest in the model with $m$ lags. The Auxiliary Result in the Appendix shows that this is true for the impulse responses. The next theorem follows from Theorem 1 and the multivariate first order delta method.

\section{Theorem 2: Asymptotic Normality of the Quantities of Interest}

Let $\boldsymbol{\mu}: \mathbb{R}^{K^{2} p+K(K+1) / 2} \rightarrow \mathbb{R}^{l}$ have a continuous first derivative at all points $\left(\boldsymbol{\theta}_{m}, \mathbf{0}_{K^{2}(p-m)}, \boldsymbol{\sigma}\right)$, with $m \in \mathcal{M}$. Let $\boldsymbol{\theta}_{\infty}$ denote the parameters obtained by taking $\boldsymbol{\theta}_{T, p}$ but setting $\boldsymbol{\Delta}_{1}=$ $\boldsymbol{\Delta}_{2}=\ldots=\boldsymbol{\Delta}_{p_{2}}=\mathbf{O}$, and define the Jacobian matrices $\boldsymbol{D}_{\theta}=\partial \boldsymbol{\mu}\left(\boldsymbol{\theta}_{\infty}, \boldsymbol{\sigma}\right) / \partial \boldsymbol{\theta}^{\prime}$ and $\boldsymbol{D}_{\sigma}=\partial \boldsymbol{\mu}\left(\boldsymbol{\theta}_{\infty}, \boldsymbol{\sigma}\right) / \partial \boldsymbol{\sigma}^{\prime}$. For $\boldsymbol{D}_{\theta}$ and $\boldsymbol{D}_{\sigma}$ not having zero rows, under Assumptions 1-3, and as $T \rightarrow \infty$,

$$
\sqrt{T}\left(\boldsymbol{\mu}\left(\left(\hat{\boldsymbol{\theta}}_{T, m}, \mathbf{0}_{K^{2}(p-m)}\right), \hat{\boldsymbol{\sigma}}\right)-\boldsymbol{\mu}\left(\boldsymbol{\theta}_{T, p}, \boldsymbol{\sigma}\right)\right) \stackrel{d}{\longrightarrow} \mathrm{N}\left(\boldsymbol{D}_{\theta} \boldsymbol{C}_{m} \boldsymbol{\delta}, \boldsymbol{D}_{\theta} \boldsymbol{P}_{m}(\boldsymbol{\Omega} \otimes \boldsymbol{\Sigma}) \boldsymbol{P}_{m} \boldsymbol{D}_{\theta}^{\prime}+\boldsymbol{D}_{\sigma} \boldsymbol{\Xi}_{22} \boldsymbol{D}_{\sigma}^{\prime}\right)
$$

with $\boldsymbol{P}_{m}=\boldsymbol{S}_{m}\left[\boldsymbol{S}_{m}^{\prime} \boldsymbol{\Omega} \boldsymbol{S}_{m}\right]^{-1} \boldsymbol{S}_{m}^{\prime} \otimes \boldsymbol{I}_{K}$ and

$$
\boldsymbol{C}_{m}=\left(\boldsymbol{S}_{m}\left[\boldsymbol{S}_{m}^{\prime} \Omega \boldsymbol{S}_{m}\right]^{-1} \boldsymbol{S}_{m}^{\prime} \boldsymbol{\Omega}-\boldsymbol{I}_{K p}\right) \boldsymbol{S}_{0}\left(\boldsymbol{I}_{K p_{2}}-\boldsymbol{\Pi}_{m}^{\prime} \boldsymbol{\Pi}_{m}\right) \otimes \boldsymbol{I}_{K}
$$

We define the impulse response at horizon $h$ as the $h^{\prime}$ th coefficient matrix of the $\operatorname{MA}(\infty)$ representation $\boldsymbol{y}_{t}=\sum_{i=0}^{\infty} \boldsymbol{\Phi}_{i} \boldsymbol{u}_{t-i}$ with $\boldsymbol{\Phi}_{0}=\boldsymbol{I}_{K}$, hence $\boldsymbol{\mu}: \mathbb{R}^{K^{2} p+K(K+1) / 2} \rightarrow \mathbb{R}^{K^{2}}$.

\footnotetext{
${ }^{4}$ This condition is a sufficient but by no means a necessary condition. For $p_{1}=1$ and $p_{2}=1$, the model $y_{T, t}=0.7 y_{T, t-1}+\frac{0.75}{\sqrt{T}} y_{T, t-2}+u_{t}$ is stationary for all $T$ but the parameters violate the requirement based on Fujiwara's bound.

${ }^{5}$ Theorem 1 showed that all the $\hat{\boldsymbol{\Sigma}}_{u}^{m}$ are asymptotically equivalent. We omit the superscript $m$ in $\hat{\boldsymbol{\Sigma}}_{u}^{m}$ from now on.
} 
Theorem 2 can be applied if the Jacobian matrices $\boldsymbol{D}_{\theta}$ and $\boldsymbol{D}_{\sigma}$ are known. Lütkepohl (1990) lists these Jacobian matrices for the impulse responses, the orthogonalized impulse responses, the accumulated responses, the total accumulated responses, and the forecast error variance decomposition. The specific case of the (orthogonalized) impulse responses is highlighted in the following Corollary.

\section{Corollary: An Application to Impulse Responses}

Let $\boldsymbol{A}_{\infty}$ denote the $(K p \times K p)$ companion matrix related to the process in which the misspecification coefficients have been set to zero, $\boldsymbol{J}=\left[\begin{array}{l}\boldsymbol{I}_{K} \\ \mathbf{O}\end{array} \cdots \mathbf{O}\right]$ a matrix of dimensions $(K \times K p)$ and $\boldsymbol{\Sigma}_{u}=\boldsymbol{P} \boldsymbol{P}^{\prime}$. Then, under the assumptions of Theorem 2,

(a) The asymptotic distribution of the estimated impulse response at horizon $i, \hat{\boldsymbol{\Phi}}_{i}$, follows

$$
\sqrt{T}\left(\operatorname{vec}\left(\hat{\boldsymbol{\Phi}}_{i}\right)-\operatorname{vec}\left(\boldsymbol{\Phi}_{i}\right)\right) \stackrel{d}{\longrightarrow} \mathrm{N}\left(\boldsymbol{G}_{i} \boldsymbol{C}_{m} \boldsymbol{\delta}, \boldsymbol{G}_{i} \boldsymbol{P}_{m}(\boldsymbol{\Omega} \otimes \boldsymbol{\Sigma}) \boldsymbol{P}_{m} \boldsymbol{G}_{i}^{\prime}\right)
$$

where $\boldsymbol{G}_{i}=\partial \operatorname{vec}\left(\boldsymbol{\Phi}_{i}\right) / \partial \boldsymbol{\theta}=\sum_{j=0}^{i-1} \boldsymbol{J}\left(\boldsymbol{A}_{\infty}\right)^{i-1-j} \otimes \boldsymbol{\Phi}_{j}$.

(b) The asymptotic distribution of the estimated orthogonalized impulse response at horizon $i, \hat{\Psi}_{i}$, follows

$$
\sqrt{T}\left(\operatorname{vec}\left(\hat{\boldsymbol{\Psi}}_{i}\right)-\operatorname{vec}\left(\boldsymbol{\Psi}_{i}\right)\right) \stackrel{d}{\longrightarrow} \mathrm{N}\left(\boldsymbol{F}_{i} \boldsymbol{C}_{m} \boldsymbol{\delta}, \boldsymbol{F}_{i} \boldsymbol{P}_{m}(\boldsymbol{\Omega} \otimes \boldsymbol{\Sigma}) \boldsymbol{P}_{m} \boldsymbol{F}_{i}^{\prime}+\overline{\boldsymbol{F}}_{i} \boldsymbol{\Xi}_{22} \overline{\boldsymbol{F}}_{i}^{\prime}\right),
$$

where $\boldsymbol{F}_{0}=\mathbf{O}$ and $\boldsymbol{F}_{i}=\left(\boldsymbol{P}^{\prime} \otimes \boldsymbol{I}_{K}\right) \boldsymbol{G}_{i}$ for $i>0$. For all $i$ we have $\overline{\boldsymbol{F}}_{i}=\left(\boldsymbol{I}_{K} \otimes \boldsymbol{\Phi}_{i}\right) \boldsymbol{H}$ with $\boldsymbol{H}=\partial \operatorname{vec}(\boldsymbol{P}) / \partial \boldsymbol{\sigma}^{\prime}=\boldsymbol{L}_{K}^{\prime}\left[\boldsymbol{L}_{K}\left(\boldsymbol{I}_{K^{2}}+\boldsymbol{K}_{K K}\right)\left(\boldsymbol{P} \otimes \boldsymbol{I}_{K}\right) \boldsymbol{L}_{K}^{\prime}\right]^{-1}$ (see Lütkepohl (1990) for the definitions of $\boldsymbol{L}_{K}$ and $\boldsymbol{K}_{K K}$ ).

Remark 2.3 The first order delta method is invalid if either $\boldsymbol{D}_{\theta}$ or $\boldsymbol{D}_{\sigma}$ has zero rows. It is well-documented in the literature that $\boldsymbol{D}_{\theta}$ can have zero rows for specific parameter combinations when impulse responses are considered. We refer to Lütkepohl (1990) and Benkwitz et al. (2000) for details.

\section{Model Selection: The Focused Information Criterion (FIC)}

The intuition behind the FIC of Claeskens and Hjort (2003) is most easily understood for a univariate quantity of interest, so we temporarily assume $l=1$. The generalization to multiple quantities is covered in Remark 2.4. Theorem 2 implies that the asymptotic mean squared error (AMSE) of the focus quantity $\mu\left(\left(\hat{\boldsymbol{\theta}}_{T, m}, \mathbf{0}_{K^{2}(p-m)}\right), \hat{\boldsymbol{\sigma}}\right)$ is given by $\operatorname{AMSE}\left(\mu\left(\left(\hat{\boldsymbol{\theta}}_{T, m}, \mathbf{0}_{K^{2}(p-m)}\right), \hat{\boldsymbol{\sigma}}\right)\right)=\boldsymbol{D}_{\theta}\left[\boldsymbol{C}_{m} \boldsymbol{\delta} \boldsymbol{\delta}^{\prime} \boldsymbol{C}_{m}^{\prime}+\boldsymbol{P}_{m}(\boldsymbol{\Omega} \otimes \boldsymbol{\Sigma}) \boldsymbol{P}_{m}\right] \boldsymbol{D}_{\theta}^{\prime}+\boldsymbol{D}_{\sigma} \boldsymbol{\Xi}_{22} \boldsymbol{D}_{\sigma}^{\prime}$. 
There are three contributions to the AMSE: (1) the term $\boldsymbol{D}_{\theta} \boldsymbol{C}_{m} \boldsymbol{\delta} \boldsymbol{\delta}^{\prime} \boldsymbol{C}_{m}^{\prime} \boldsymbol{D}_{\theta}^{\prime}$ is an asymptotic squared bias originating from the exclusion of local-to-zero parameters, (2) the asymptotic variance contribution $\boldsymbol{D}_{\theta} \boldsymbol{P}_{m}(\boldsymbol{\Omega} \otimes \boldsymbol{\Sigma}) \boldsymbol{P}_{m} \boldsymbol{D}_{\theta}^{\prime}$, and (3) the contribution $\boldsymbol{D}_{\sigma} \boldsymbol{\Xi}_{22} \boldsymbol{D}_{\sigma}^{\prime}$ which does not depend on the lag order $m$. Overall we face a bias-variance tradeoff when having to decide on $m$.

The FIC is an estimate of the AMSE. The quantities $\hat{\boldsymbol{\theta}}_{T, p}$ and $\hat{\boldsymbol{\Omega}}=\frac{1}{T} \sum_{t=1}^{T} \boldsymbol{z}_{T, t} \boldsymbol{z}_{T, t}^{\prime}$ provide consistent estimates for $\boldsymbol{\theta}_{T}$ and $\boldsymbol{\Omega}$, repectively. In view of the continuous mapping theorem, $\hat{\boldsymbol{P}}_{m}=\boldsymbol{S}_{m}\left[\boldsymbol{S}_{m}^{\prime} \hat{\Omega} \boldsymbol{S}_{m}\right]^{-1} \boldsymbol{S}_{m}^{\prime} \otimes \boldsymbol{I}_{K}$ and

$$
\hat{\boldsymbol{C}}_{m}=\left(\boldsymbol{S}_{m}\left[\boldsymbol{S}_{m}^{\prime} \hat{\Omega} \boldsymbol{S}_{m}\right]^{-1} \boldsymbol{S}_{m}^{\prime} \hat{\Omega}-\boldsymbol{I}_{K p}\right) \boldsymbol{S}_{0}\left(\boldsymbol{I}_{K p_{2}}-\boldsymbol{\Pi}_{m}^{\prime} \boldsymbol{\Pi}_{m}\right) \otimes \boldsymbol{I}_{K}
$$

are consistent estimators as well. A consistent estimator for $\delta$ is not available due to the adopted misspecification framework. We follow the existing literature (see Claeskens and Hjort (2003), Liu (2015), and Charkhi et al. (2016) among others) and use $\hat{\delta}=$ $\sqrt{T} \operatorname{vec}\left(\hat{\Theta}_{T, p} \boldsymbol{S}_{0}\right)$ which satisfies ${ }^{6}$

$$
\hat{\boldsymbol{\delta}} \stackrel{d}{\longrightarrow} \boldsymbol{R}_{\delta}=\boldsymbol{\delta}+\left(\boldsymbol{S}_{0}^{\prime} \Omega^{-1} \otimes \boldsymbol{I}_{K}\right) \boldsymbol{R} \sim \mathrm{N}\left(\boldsymbol{\delta}, \boldsymbol{S}_{0}^{\prime} \Omega^{-1} \boldsymbol{S}_{0} \otimes \Sigma\right) .
$$

Asymptotically, we have $\mathrm{E}\left(\hat{\boldsymbol{\delta}} \hat{\boldsymbol{\delta}}^{\prime}\right)=\boldsymbol{\delta} \boldsymbol{\delta}^{\prime}+\boldsymbol{S}_{0}^{\prime} \boldsymbol{\Omega}^{-1} \boldsymbol{S}_{0} \otimes \Sigma$. Using the asymptotically unbiased estimate $\hat{\delta} \hat{\boldsymbol{\delta}}^{\prime}-\boldsymbol{S}_{0}^{\prime} \hat{\Omega}^{-1} \boldsymbol{S}_{0} \otimes \hat{\Sigma}$ for $\boldsymbol{\delta} \boldsymbol{\delta}^{\prime}$, the FIC for the approximating model with $m$ lags is defined as

$$
\widehat{F I C}_{m}=D_{\theta}\left[\hat{C}_{m}\left(\hat{\delta} \hat{\delta}^{\prime}-S_{0}^{\prime} \hat{\Omega}^{-1} \boldsymbol{S}_{0} \otimes \hat{\Sigma}\right) \hat{C}_{m}^{\prime}+\hat{\boldsymbol{P}}_{m}(\hat{\Omega} \otimes \hat{\Sigma}) \hat{\boldsymbol{P}}_{m}\right] \boldsymbol{D}_{\theta}^{\prime}+\boldsymbol{D}_{\sigma} \hat{\Xi}_{22} \boldsymbol{D}_{\sigma}^{\prime}
$$

This estimate of the AMSE can be computed for every model, and the model with the lowest $\widehat{F I C}_{m}$ is selected. We elaborate on implications of inconsistent estimation of $\boldsymbol{\delta}$ in Section 2.2.4.

Remark 2.4 The same procedure can be followed when $l>1$, but the AMSE becomes an $(l \times l)$ matrix. The trace or determinant are meaningful ways to describe this AMSE matrix by a scalar (see Charkhi et al. (2016)). ${ }^{7}$ The trace is computationally convenient because the overall FIC will be the sum of the individual univariate FIC contributions.

\footnotetext{
${ }^{6} \hat{\boldsymbol{\delta}}=\sqrt{T} \operatorname{vec}\left(\hat{\boldsymbol{\Theta}}_{T, p} \boldsymbol{S}_{0}\right)$ is the sample equivalent of $\boldsymbol{\delta}=\operatorname{vec}(\boldsymbol{\Delta})=\sqrt{T} \operatorname{vec}\left(\boldsymbol{C}_{T}\right)=\sqrt{T} \operatorname{vec}\left(\boldsymbol{\Theta}_{T, p} \boldsymbol{S}_{0}\right)$.

${ }^{7}$ Any mapping from the AMSE matrix to a scalar can be used, e.g. matrix norms could be used as well.
} 


\section{Model Averaging: Plug-in Averaging}

Liu (2015) proposed a model averaging approach along the lines of the FIC. It was named plug-in averaging. We again depart from the case $l=1$, see Remark 2.5 for the generalization. Part (b) from Theorem 1 implies that linear combinations of the VAR parameter estimators are also asymptotically normally distributed. The coefficients in the linear combination can be interpreted as weights, i.e. define $\boldsymbol{w}=\left(w_{p_{1}}, w_{p_{1}+1}, \ldots, w_{p}\right)$ with $\boldsymbol{w} \in \mathcal{H}=\left\{\boldsymbol{w} \in[0,1]^{p_{2}+1}: \sum_{m=p_{1}}^{p} w_{m}=1\right\} .{ }^{8}$

Theorem 3 details the asymptotic distribution of the following weighted estimator,

$$
\bar{\mu}(\boldsymbol{w})=\sum_{m=p_{1}}^{p} w_{m} \mu\left(\left(\hat{\boldsymbol{\theta}}_{T, m}, \mathbf{0}_{K^{2}(p-m)}\right), \hat{\boldsymbol{\sigma}}\right) .
$$

\section{Theorem 3: Asymptotic Normality of the Plug-In Estimator}

Under the Assumptions of Theorem 2, we have for $T \rightarrow \infty$,

$$
\begin{aligned}
\sqrt{T}\left(\bar{\mu}(\boldsymbol{w})-\mu\left(\boldsymbol{\theta}_{T}, \boldsymbol{\sigma}\right)\right) & \stackrel{d}{\longrightarrow} \boldsymbol{D}_{\theta} \sum_{m=p_{1}}^{p} w_{m} \boldsymbol{C}_{m} \boldsymbol{\delta}+\boldsymbol{D}_{\theta} \sum_{m=p_{1}}^{p} w_{m} \boldsymbol{P}_{m} \boldsymbol{R}+\boldsymbol{D}_{\sigma} \boldsymbol{S} \\
& \sim \mathrm{N}\left(\boldsymbol{D}_{\theta} \sum_{m=p_{1}}^{p} w_{m} \boldsymbol{C}_{m} \boldsymbol{\delta}, \boldsymbol{V}+\boldsymbol{D}_{\sigma} \boldsymbol{\Xi}_{22} \boldsymbol{D}_{\sigma}^{\prime}\right),
\end{aligned}
$$

with $\boldsymbol{V}=\sum_{m=p_{1}}^{p} \sum_{l=p_{1}}^{p} w_{m} w_{l} \boldsymbol{D}_{\theta} \boldsymbol{P}_{m}(\boldsymbol{\Omega} \otimes \boldsymbol{\Sigma}) \boldsymbol{P}_{l} \boldsymbol{D}_{\theta}^{\prime}$.

As for the FIC, we compute the AMSE and find ${ }^{9}$

$$
\operatorname{AMSE}(\bar{\mu}(\boldsymbol{w}))=\sum_{m=p_{1}}^{p} \sum_{l=p_{1}}^{p} w_{m} \boldsymbol{D}_{\theta}\left(\boldsymbol{C}_{m} \boldsymbol{\delta} \boldsymbol{\delta}^{\prime} \boldsymbol{C}_{l}^{\prime}+\boldsymbol{P}_{m}(\boldsymbol{\Omega} \otimes \boldsymbol{\Sigma}) \boldsymbol{P}_{l}\right) \boldsymbol{D}_{\theta}^{\prime} w_{l}=\boldsymbol{w}^{\prime} \boldsymbol{\Psi} \boldsymbol{w}
$$

with the $\left(\left(p_{2}+1\right) \times\left(p_{2}+1\right)\right)$ matrix $\boldsymbol{\Psi}$ having the $(m, l)$ 'th element $\boldsymbol{\Psi}_{m, l}=\boldsymbol{D}_{\theta}\left(\boldsymbol{C}_{m} \boldsymbol{\delta} \boldsymbol{\delta}^{\prime} \boldsymbol{C}_{l}^{\prime}+\right.$ $\left.\boldsymbol{P}_{m}(\boldsymbol{\Omega} \otimes \boldsymbol{\Sigma}) \boldsymbol{P}_{l}\right) \boldsymbol{D}_{\theta}^{\prime}$. The optimal weight vector in terms of minimal AMSE is $\boldsymbol{w}^{0}=$ $\arg \min _{\boldsymbol{w} \in \mathcal{H}} \boldsymbol{w}^{\prime} \boldsymbol{\Psi} \boldsymbol{w}$. But $\boldsymbol{w}^{0}$ depends on population quantities, so using the same estimates for population quantities as before, we compute feasible weights as

$$
\hat{\boldsymbol{w}}=\arg \min _{\boldsymbol{w} \in \mathcal{H}} \boldsymbol{w}^{\prime} \hat{\boldsymbol{\Psi}}^{j} \boldsymbol{w}, \quad j \in\{\text { Biased, Bias cor }\}
$$

\footnotetext{
${ }^{8}$ We assume that we average over all the models in $\mathcal{M}=\left\{p_{1}, p_{1}+1, \ldots, p\right\}$.

${ }^{9}$ The contribution $\boldsymbol{D}_{\sigma} \boldsymbol{\Xi}_{22} \boldsymbol{D}_{\sigma}^{\prime}$ does not depend on $m$, and is therefore inconsequential for the analysis. This term will be omitted from the AMSE to allow for an easier presentation.
} 
with

$$
\begin{aligned}
\hat{\boldsymbol{\Psi}}_{m, l}^{\text {Biased }} & =\boldsymbol{D}_{\theta}\left[\hat{\boldsymbol{C}}_{m} \hat{\boldsymbol{\delta}} \hat{\boldsymbol{\delta}}^{\prime} \hat{\boldsymbol{C}}_{l}^{\prime}+\hat{\boldsymbol{P}}_{m}(\hat{\boldsymbol{\Omega}} \otimes \hat{\boldsymbol{\Sigma}}) \hat{\boldsymbol{P}}_{l}\right] \boldsymbol{D}_{\theta}^{\prime}, \\
\hat{\boldsymbol{\Psi}}_{m, l}^{\text {Bias cor }} & =\boldsymbol{D}_{\theta}\left[\hat{\boldsymbol{C}}_{m}\left(\hat{\boldsymbol{\delta}} \hat{\boldsymbol{\delta}}^{\prime}-\boldsymbol{S}_{0}^{\prime} \hat{\boldsymbol{\Omega}}^{-1} \boldsymbol{S}_{0} \otimes \hat{\boldsymbol{\Sigma}}\right) \hat{\boldsymbol{C}}_{l}^{\prime}+\hat{\boldsymbol{P}}_{m}(\hat{\boldsymbol{\Omega}} \otimes \hat{\boldsymbol{\Sigma}}) \hat{\boldsymbol{P}}_{l}\right] \boldsymbol{D}_{\theta}^{\prime} .
\end{aligned}
$$

The sole difference between the matrix elements in Equation (2.15) is an asymptotic bias correction for $\hat{\boldsymbol{\delta}} \hat{\boldsymbol{\delta}}^{\prime}$. Equation (2.14) is a quadratic programming problem with linear constraints. Solvers are readily available (e.g. 'quadprog' in Matlab). The estimator for $\boldsymbol{\delta}$ remains inconsistent and we refer to Section 2.2.4 for a discussion of the implications.

Remark 2.5 As in Remark 2.4, we will obtain an $(l \times l)$ AMSE matrix for multiple quantities of interest. This matrix has to be summarized by a scalar. The trace has again computational benefits because the objective function will take the form $\hat{\boldsymbol{w}}=$ $\arg \min _{\boldsymbol{w} \in \mathcal{H}} \boldsymbol{w}^{\prime}\left(\sum_{i} \hat{\boldsymbol{\Psi}}_{i}^{j}\right) \boldsymbol{w}$ with $\hat{\boldsymbol{\Psi}}_{i}^{j}$ the matrix corresponding to the i'th focus quantity.

Remark 2.6 Two remarks related to Charkhi et al. (2016) are in place.

1. The weight vector $\hat{\boldsymbol{w}}$ is only uniquely determined when $\hat{\boldsymbol{\Psi}}^{j}$ is positive definite. As such, the bias subtraction may lead to non-unique weights.

2. Charkhi et al. (2016) consider a weighting scheme in which the weights sum to one but are not necessarily positive. Simulation results have shown that it is advisable to keep the positivity constraint in our autoregressive setup because weights can otherwise become large in magnitude and unstable.

Remark 2.7 Autoregressive models of infinite order have been considered by Berk (1974) and Lewis and Reinsel (1985) among others. It is an intriguing question whether the current framework can be extended to $V A R(\infty)$ models. ${ }^{10}$ We argue that the main difficulty is the estimation of the infinitely many local-to-zero parameters. Let us consider the univariate model $y_{T, t}=\alpha y_{T, t-1}+\sum_{j=1}^{\infty}\left(\frac{\delta_{j}}{\sqrt{T}}\right) y_{T, t-1-j}+u_{t}$ as an illustration. We conjecture ${ }^{11}$ that the asymptotic distribution of the approximating $A R(1)$ model follows $\sqrt{T}(\hat{\alpha}-\alpha) \stackrel{d}{\longrightarrow}$ $\mathrm{N}\left(\sum_{j=1}^{\infty} \delta_{j} \alpha^{j}, 1-\alpha^{2}\right)$. The bias contribution to the AMSE now depends on infinitely many $\delta_{j}$. Their estimation would require the lag order of the largest approximating model to grow with sample size. Our proof of Theorem 1 does not easily allow for such an extension

\footnotetext{
${ }^{10}$ We thank an anonymous referee for bringing this topic to our attention.

${ }^{11}$ We can be more precise concerning our assumptions. Theorem 1 has shown that the asymptotic results are governed by the process with the local-to-zero parameters set equal to zero. We assume that this remains true when there are infinitely many local-to-zero parameters.
} 
since we currently rely on the finite dimension of the companion matrix. A full exploration of this topic is left for further research.

There is one final result that forces us to look at the case $l>1$. Practitioners are usually interested in the impulse responses for several horizons. Using a separate weight vector for every horizon may: (1) create impulse responses that vary irregularly from one horizon to the next due to strong changes in the weights, and (2) result in confidence intervals that do not take into account the dependence between the horizons. Theorem 4 below extends the result of Liu (2015) to obtain asymptotically valid confidence bands for several horizons.

\section{Theorem 4: Joint Confidence Bands}

Under the Assumptions of Theorem 2, if $w_{m}(\hat{\boldsymbol{\delta}}) \stackrel{d}{\longrightarrow} w_{m}\left(\boldsymbol{R}_{\delta}\right)$, and if $\boldsymbol{D}_{\theta}(\boldsymbol{\Omega} \otimes \boldsymbol{\Sigma}) \boldsymbol{D}_{\theta}^{\prime}+$ $\boldsymbol{D}_{\sigma} \boldsymbol{\Xi}_{22} \boldsymbol{D}_{\sigma}>0$, then

$$
\begin{array}{r}
\left(\sqrt{T}\left(\overline{\boldsymbol{\mu}}(\hat{\boldsymbol{w}})-\boldsymbol{\mu}\left(\boldsymbol{\theta}_{T}, \boldsymbol{\sigma}\right)\right)-\boldsymbol{D}_{\theta} \sum_{m=p_{1}}^{p} \hat{w}_{m} \hat{\boldsymbol{C}}_{m} \hat{\boldsymbol{\delta}}\right)^{\prime}\left(\boldsymbol{D}_{\theta}(\hat{\boldsymbol{\Omega}} \otimes \hat{\boldsymbol{\Sigma}}) \boldsymbol{D}_{\theta}^{\prime}+\boldsymbol{D}_{\sigma} \hat{\boldsymbol{\Xi}}_{22} \boldsymbol{D}_{\sigma}\right)^{-1} \\
\left(\sqrt{T}\left(\overline{\boldsymbol{\mu}}(\hat{\boldsymbol{w}})-\boldsymbol{\mu}\left(\boldsymbol{\theta}_{T}, \boldsymbol{\sigma}\right)\right)-\boldsymbol{D}_{\theta} \sum_{m=p_{1}}^{p} \hat{w}_{m} \hat{\boldsymbol{C}}_{m} \hat{\boldsymbol{\delta}}\right) \leq \chi_{l, 1-\alpha}^{2},
\end{array}
$$

is an asymptotically correct $(1-\alpha)$ confidence band, where $\chi_{l, 1-\alpha}^{2}$ denotes the $(1-\alpha)$ quantile of a chi-squared distributed random variable with l degrees of freedom.

Remark 2.8 There is one practical concern which has not been addressed, namely the choices for $p_{1}$ and $p{ }^{12} p_{1}$ turns out to be unimportant. To see this, we consider the expression for $\widehat{F I C}_{m}$ (a similar reasoning applies to the plug-in weights). The terms $\boldsymbol{D}_{\theta} \hat{\boldsymbol{P}}_{m}(\hat{\boldsymbol{\Omega}} \otimes \hat{\boldsymbol{\Sigma}}) \hat{\boldsymbol{P}}_{m} \boldsymbol{D}_{\theta}^{\prime}$ and $\boldsymbol{D}_{\sigma} \hat{\boldsymbol{\Xi}}_{22} \boldsymbol{D}_{\sigma}^{\prime}$ in Equation (2.11) do not depend on $p_{1}$, so it remains to inspect the contribution $D_{\theta} \hat{C}_{m}\left(\hat{\delta} \hat{\boldsymbol{\delta}}^{\prime}-\boldsymbol{S}_{0}^{\prime} \hat{\Omega}^{-1} \boldsymbol{S}_{0} \otimes \hat{\Sigma}\right) \hat{C}_{m}^{\prime} \boldsymbol{D}_{\theta}^{\prime}$. Using $\hat{\boldsymbol{\delta}}=$ $\sqrt{T}\left(\boldsymbol{S}_{0}^{\prime} \otimes \boldsymbol{I}_{K}\right) \hat{\boldsymbol{\theta}}_{T, p}$ we can rewrite this contribution as

$$
\boldsymbol{D}_{\theta}\left(\hat{C}_{m}\left(\boldsymbol{S}_{0}^{\prime} \otimes \boldsymbol{I}_{K}\right)\right)\left[\left(\sqrt{T} \hat{\boldsymbol{\theta}}_{T, p}\right)\left(\sqrt{T} \hat{\boldsymbol{\theta}}_{T, p}\right)^{\prime}-\hat{\boldsymbol{\Omega}}^{-1} \otimes \hat{\boldsymbol{\Sigma}}\right]\left(\hat{\boldsymbol{C}}_{m}\left(\boldsymbol{S}_{0}^{\prime} \otimes \boldsymbol{I}_{K}\right)\right)^{\prime} \boldsymbol{D}_{\theta}^{\prime}
$$

\footnotetext{
${ }^{12}$ We thank an anonymous referee who rightfully conjectured that the choice of $p_{1}$ does not have an influence on the numerical outcome for $\widehat{F I C}_{m}$.
} 
By definition of $\hat{\boldsymbol{C}}_{m}$, we have

$$
\begin{aligned}
\hat{\boldsymbol{C}}_{m}\left(\boldsymbol{S}_{0}^{\prime} \otimes \boldsymbol{I}_{K}\right) & =\left(\boldsymbol{S}_{m}\left[\boldsymbol{S}_{m}^{\prime} \hat{\boldsymbol{\Omega}} \boldsymbol{S}_{m}\right]^{-1} \boldsymbol{S}_{m}^{\prime} \hat{\boldsymbol{\Omega}}-\boldsymbol{I}_{K p}\right)\left[\boldsymbol{S}_{0}\left(\boldsymbol{I}_{K p_{2}}-\boldsymbol{\Pi}_{m}^{\prime} \boldsymbol{\Pi}_{m}\right) \boldsymbol{S}_{0}^{\prime}\right] \otimes \boldsymbol{I}_{K} \\
& =\left(\boldsymbol{S}_{m}\left[\boldsymbol{S}_{m}^{\prime} \hat{\boldsymbol{\Omega}} \boldsymbol{S}_{m}\right]^{-1} \boldsymbol{S}_{m}^{\prime} \hat{\boldsymbol{\Omega}}-\boldsymbol{I}_{K p}\right)(\underbrace{\left.\left[\begin{array}{cc}
\mathbf{O} & \mathbf{O} \\
\mathbf{O} & \boldsymbol{I}_{K(p-m)}
\end{array}\right] \otimes \boldsymbol{I}_{K}\right)}_{\mathcal{B}},
\end{aligned}
$$

thereby showing that actually none of the contributions to $\widehat{F I C}_{m}$ depends on $p_{1}$. However, the zero pattern of the matrix $\mathcal{B}$ in Equation (2.17) will only cause a non-diverging value for $\widehat{F I C}_{m}$ if models are chosen such that $m \in \mathcal{M}=\left\{p_{1}, p_{1}+1, \ldots, p\right\}$. This supports the claim in Remark 2.1. The lag order of the full model, that is p, might be chosen by AIC or set equal to an a priori selected $p_{\max }$.

\subsubsection{The Effects of Inconsistently Estimating Delta}

Equation (2.10) showed that $\hat{\boldsymbol{\delta}}$ converges to a normally distributed random vector centered around $\delta$. How does this influence the selection and averaging procedures? Clearly, $\widehat{F I C}_{m}, \hat{\Psi}_{m, l}^{\text {Biased }}$, and $\hat{\Psi}_{m, l}^{\text {Bias cor }}$ will not converge in probability to the AMSE they are intended to estimate. The limiting distributions of these quantities are highlighted in Theorems 5 and 6 . The plug-in results are stated for $\hat{\Psi}_{m, l}^{\text {Bias cor }}$, but a simple omission of the bias correction term would give the corresponding findings for $\hat{\Psi}_{m, l}^{\text {Biased }}$.

\section{Theorem 5: The Asymptotic Behavior of $\widehat{F I C}_{m}$}

Under the Assumptions of Theorem 2, we have for $m \in \mathcal{M} \backslash p$,

$$
\begin{aligned}
\widehat{F I C}_{m} & \stackrel{d}{\longrightarrow} \boldsymbol{D}_{\theta}\left[\boldsymbol{C}_{m}\left(\boldsymbol{R}_{\delta} \boldsymbol{R}_{\delta}^{\prime}-\left(\boldsymbol{S}_{0}^{\prime} \boldsymbol{\Omega} \boldsymbol{S}_{0} \otimes \Sigma\right)\right) \boldsymbol{C}_{m}^{\prime}+\boldsymbol{P}_{m}(\boldsymbol{\Omega} \otimes \boldsymbol{\Sigma}) \boldsymbol{P}_{m}\right] \boldsymbol{D}_{\theta}^{\prime}+\boldsymbol{D}_{\sigma} \boldsymbol{\Xi}_{22} \boldsymbol{D}_{\sigma}^{\prime} \\
& :=F I C_{m}^{\infty} \\
& \sim a_{m} \chi_{\text {noncentral }}^{2}\left(1,\left(\boldsymbol{D}_{\theta} \boldsymbol{C}_{m} \boldsymbol{\delta}\right)^{2} / a_{m}\right)-a_{m}+\boldsymbol{D}_{\theta} \boldsymbol{P}_{m}(\boldsymbol{\Omega} \otimes \boldsymbol{\Sigma}) \boldsymbol{P}_{m} \boldsymbol{D}_{\theta}^{\prime}+\boldsymbol{D}_{\sigma} \boldsymbol{\Xi}_{22} \boldsymbol{D}_{\sigma}^{\prime}
\end{aligned}
$$

where $a_{m}=\boldsymbol{D}_{\theta} \boldsymbol{C}_{m}\left(\boldsymbol{S}_{0}^{\prime} \boldsymbol{\Omega}^{-1} \boldsymbol{S}_{0} \otimes \boldsymbol{\Sigma}\right) \boldsymbol{C}_{m}^{\prime} \boldsymbol{D}_{\theta}^{\prime}$, and $\chi_{\text {noncentral }}^{2}(v, \lambda)$ denotes a noncentral chisquared distributed random variable with $v$ degrees of freedom and noncentrality parameter $\lambda$. It can be shown that $\mathrm{E}\left(F I C_{m}^{\infty}\right)=\operatorname{AMSE}\left(\mu\left(\hat{\boldsymbol{\theta}}_{T, m}, \mathbf{0}_{K^{2}(p-m)}, \hat{\boldsymbol{\sigma}}\right)\right)$ and $\operatorname{var}\left(F I C_{m}^{\infty}\right)=$ $2 a_{m}\left(a_{m}+2\left(\boldsymbol{D}_{\theta} \boldsymbol{C}_{m} \boldsymbol{\delta}\right)^{2}\right)$. For the full model, $m=p$, we have

$$
\widehat{F I C}_{p} \stackrel{p}{\longrightarrow} F I C_{p}^{\infty}=\boldsymbol{D}_{\theta}\left(\Omega^{-1} \otimes \boldsymbol{\Sigma}\right) \boldsymbol{D}_{\theta}^{\prime}=\operatorname{AMSE}\left(\mu\left(\hat{\boldsymbol{\theta}}_{T, p}, \hat{\boldsymbol{\sigma}}\right)\right)
$$


Theorem 6: The Asymptotic Behavior of $\hat{\Psi}_{m, l}^{\text {Biased }}$ and $\hat{\Psi}_{m, l}^{\text {Bias cor }}$ Under the Assumptions of Theorem 2, we have for $m, l \in \mathcal{M} \backslash p$,

$$
\begin{aligned}
\hat{\Psi}_{m, l}^{\text {Bias cor }} & \stackrel{d}{\longrightarrow} \boldsymbol{R}_{\delta}^{\prime} C_{m}^{\prime} D_{\theta}^{\prime} \boldsymbol{D}_{\theta} C_{l} \boldsymbol{R}_{\delta}+\boldsymbol{D}_{\theta} \boldsymbol{P}_{m}(\Omega \otimes \Sigma) \boldsymbol{P}_{l} \boldsymbol{D}_{\theta}^{\prime}-\boldsymbol{D}_{\theta} \boldsymbol{C}_{m}\left(\boldsymbol{S}_{0}^{\prime} \Omega \boldsymbol{S}_{0} \otimes \Sigma\right) \boldsymbol{C}_{m}^{\prime} \boldsymbol{D}_{\theta}^{\prime} \\
& :=\boldsymbol{\Psi}_{m, l}^{\text {Bias cor }, \infty} .
\end{aligned}
$$

Two cases can be distinguished:

(a) If $m=l$, then $\boldsymbol{\Psi}_{m, m}^{\text {Bias cor, } \infty} \sim a_{m} \chi_{\text {noncentral }}^{2}\left(1,\left(\boldsymbol{D}_{\theta} \boldsymbol{C}_{m} \boldsymbol{\delta}\right)^{2} / a_{m}\right)-a_{m}+\boldsymbol{D}_{\theta} \boldsymbol{P}_{m}(\boldsymbol{\Omega} \otimes$ $\boldsymbol{\Sigma}) \boldsymbol{P}_{m} \boldsymbol{D}_{\theta}^{\prime}$

(b) Define $\mathcal{A}=S_{0}^{\prime} \Omega^{-1} S_{0} \otimes \Sigma$, and consider the eigenvalue decomposition given by $\frac{1}{2} \mathcal{A}^{1 / 2}\left(\boldsymbol{C}_{m}^{\prime} \boldsymbol{D}_{\theta} \boldsymbol{D}_{\theta}^{\prime} \boldsymbol{C}_{l}+\boldsymbol{C}_{l}^{\prime} \boldsymbol{D}_{\theta} \boldsymbol{D}_{\theta}^{\prime} \boldsymbol{C}_{m}\right) \mathcal{A}^{1 / 2}=\sum_{i=1}^{2} \lambda_{i} \boldsymbol{v}_{i} \boldsymbol{v}_{i}^{\prime}$, where $\lambda_{i}$ denotes the eigenvalue corresponding to the eigenvector $\boldsymbol{v}_{i}$. If $m \neq l$, then

$$
\begin{gathered}
\boldsymbol{\Psi}_{m, l}^{\text {Bias cor, } \infty} \sim \sum_{i=1}^{2} \lambda_{i} \chi_{\text {noncentral }}^{2}\left(1,\left(\boldsymbol{v}_{i}^{\prime} \mathcal{A}^{-1 / 2} \boldsymbol{\delta}\right)^{2}\right)+\boldsymbol{D}_{\theta} \boldsymbol{P}_{m}(\boldsymbol{\Omega} \otimes \boldsymbol{\Sigma}) \boldsymbol{P}_{l} \boldsymbol{D}_{\theta}^{\prime} \\
-\boldsymbol{D}_{\theta} \boldsymbol{C}_{m}\left(\boldsymbol{S}_{0}^{\prime} \boldsymbol{\Omega} \boldsymbol{S}_{0} \otimes \boldsymbol{\Sigma}\right) \boldsymbol{C}_{m}^{\prime} \boldsymbol{D}_{\theta}^{\prime}
\end{gathered}
$$

If $m=p$ and/or $l=p$, then $\hat{\Psi}_{m, l}^{\text {Bias cor }} \stackrel{p}{\longrightarrow} \Psi_{m, l}$.

Theorems 5 and 6 stated the limiting distribution of the FIC and the matrix elements that enter the weighting scheme. Based on the random limits of these quantities, we might expect that our methods will not truly minimize the AMSE among either model choices or model weights. We proceed with a small illustration to stress the difference between knowing $\boldsymbol{\delta}$ and having an estimator $\hat{\boldsymbol{\delta}}$ that converges in distribution only.

\section{An Illustration}

Consider a simplified DGP, $y_{T, t}=\alpha y_{T, t-1}+\frac{\delta}{\sqrt{T}} y_{T, t-2}+u_{t}$, with $\operatorname{var}\left(u_{t}\right)=1$, and a focus on the impulse response at horizon 1 (i.e. $\boldsymbol{D}_{\theta}=(1,0)$ and $\left.\boldsymbol{D}_{\sigma}=0\right)$. The model set is $\mathcal{M}=\{1,2\}$. This simplified setting makes the asymptotic behavior of the FIC and plug-in weights analytically tractable. Details of computations can be found in Appendix 2.B. Figure 2.1(a) depicts $F I C_{1}^{\infty}$ and $F I C_{2}^{\infty}$ as a function of $\delta$. Note that $F I C_{1}^{\infty}$ converges in distribution and has a nonzero probability to give an outcome below $F I C_{2}^{\infty}$. This asymptotic selection probability of the model with $m=1$ can be calculated analytically using Theorem 5. Figure 2.1(b) shows that the FIC does not select the model with the smallest AMSE with probability one. 
Our simplified model can also be used to examine the effect of $\hat{\delta}$ on the plug-in weights. We focus on the weights in the absence of bias correction..$^{13}$ The $(2 \times 2)$ limiting matrix $\Psi^{\infty}$ is

$$
\boldsymbol{\Psi}^{\infty}=\left[\begin{array}{cc}
a_{1} \chi_{\text {noncentral }}^{2}\left(1,\left(\boldsymbol{D}_{\theta} \boldsymbol{C}_{1} \boldsymbol{\delta}\right)^{2} / a_{1}\right)+\sigma^{2} \boldsymbol{D}_{\theta} \boldsymbol{P}_{1} \boldsymbol{D}_{\theta}^{\prime} & \sigma^{2} \boldsymbol{D}_{\theta} \boldsymbol{P}_{1} \boldsymbol{D}_{\theta}^{\prime} \\
\sigma^{2} \boldsymbol{D}_{\theta} \boldsymbol{P}_{1} \boldsymbol{D}_{\theta}^{\prime} & \sigma^{2} \boldsymbol{D}_{\theta} \boldsymbol{\Omega}^{-1} \boldsymbol{D}_{\theta}^{\prime}
\end{array}\right],
$$

where $a_{1}=\sigma^{2} \boldsymbol{D}_{\theta} \boldsymbol{C}_{1} \boldsymbol{S}_{0}^{\prime} \Omega \boldsymbol{S}_{0} \boldsymbol{C}_{1}^{\prime} \boldsymbol{D}_{\theta}^{\prime}$ (see Theorem 6). Let $w^{*}$ denote the asymptotically optimal plug-in weight for the model with $m=1$. We have

$$
\operatorname{Pr}\left(w^{*} \leq x\right)=\operatorname{Pr}\left(\chi_{\text {noncentral }}^{2}\left(1,\left(\boldsymbol{D}_{\theta} \boldsymbol{C}_{1} \boldsymbol{\delta}\right)^{2} / a_{1}\right) \geq \frac{\sigma^{2} \boldsymbol{D}_{\theta}\left(\boldsymbol{\Omega}^{-1}-\boldsymbol{P}_{1}\right) \boldsymbol{D}_{\theta}^{\prime}[1-x]}{a_{1} x}\right) .
$$

Figure 2.2 shows the area between the 5\% and 95\% quantiles of $w^{*}$ together with the optimal weight for known delta. We see that the asymptotic distribution of $w^{*}$ is located closer to zero than the optimal infeasible weights. This is unsurprising because the lack of bias-correction causes (on average) an overestimation of the AMSE of the model with $m=1$.

Remark 2.9 The exposition in this section was based on a simplified model. We concluded that the absence of a consistent estimator for $\delta$ translates into suboptimal model selection and suboptimal model averaging. Also in more elaborate models, the FIC and the elements of the weighting matrix $\boldsymbol{\Psi}$ will converge to random variables (except for $m=p$ ). It is key to realize that the AMSE with estimated $\delta$ will not coincide with the AMSE that can be attained if $\boldsymbol{\delta}$ was either known or consistently estimated. We conjecture that these considerations are equally relevant outside an autoregressive framework, e.g. in the regression framework discussed in Liu (2015) and the likelihood framework of Charkhi et al. (2016).

\footnotetext{
${ }^{13}$ There is a finite probability for the matrix $\boldsymbol{\Phi}^{\infty}$ to have a negative eigenvalue when the bias correction is applied. This severely complicates the derivations, so we exclude this case from our analysis. For the $\boldsymbol{\Psi}^{\infty}$ matrix without bias correction we will have $\boldsymbol{\Psi}^{\infty}>0$ if $\boldsymbol{D}_{\theta}^{\prime}\left(\boldsymbol{\Omega}^{-1}-\boldsymbol{P}_{1}\right) \boldsymbol{D}_{\theta}>0$. The latter requirement is equivalent to $\boldsymbol{D}_{\theta}^{\prime}\left[\begin{array}{c}-\omega_{12} \\ \omega_{11}\end{array}\right] \neq 0$.
} 


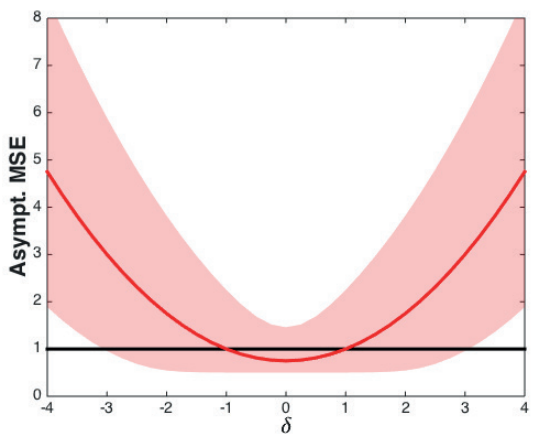

(a)

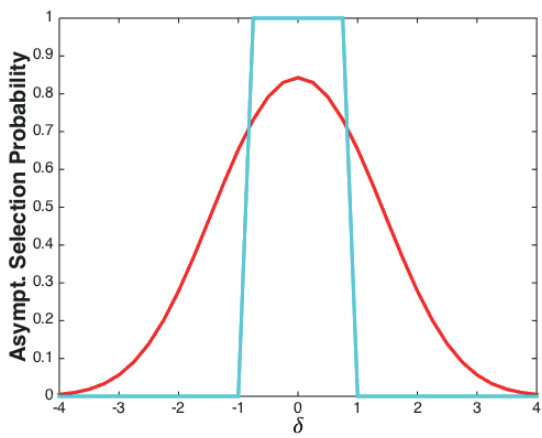

(b)

Figure 2.1: (a) The asymptotic MSE of the models with one and two lags (red and black line, respectively). The area between the $5 \%$ and $95 \%$ quantiles of $F I C_{1}^{\infty}$ is shaded in red. $\widehat{F I C}_{2}$ converges in probability to the values of the black line. (b) The asymptotic selection probabilities of the model with $m=1$. The infeasible estimator takes a binary decision based on whether the red or black line in graph (a) is lowest. Model selection based on the Focused Information Criterion results in a smoothed asymptotic selection probability because $\widehat{F I C}_{1}$ converges in distribution.

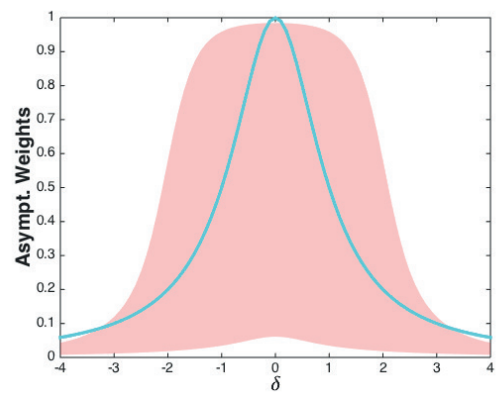

Figure 2.2: The 5\% and 95\% quantiles of the asymptotic distribution of the weights as a shaded red area, see Equation (2.19). The solid cyan lines are the asymptotically optimal weights which can only be obtained if either $\delta$ is known (infeasible) or a consistent estimator for $\delta$ is available. 


\subsection{Simulation Results}

This simulation section consists of three parts. ${ }^{14}$ In the first part we will verify our derivations for the simplified DGP and see how suboptimal selection/averaging affects the finite sample MSE. This section is followed by a study of the impulse responses for different horizons in a univariate and multivariate setting. All our graphs are made as a function of the scalar $\delta$. This scalar measures the amount of misspecification and the closeness to unit root. ${ }^{15}$ Any missing starting values in the autoregressive recursion were replaced by zeros, and the first 100 data points were omitted as a presample. All results are based on 100,000 Monte Carlo replications.

The performance of the various methods was assessed using the empirical mean squared error. For model selection the featured methods are:

1. The Akaike information criterion ('AIC') and Bayesian information criterion ('BIC'), e.g. section 4.3 of Lütkepohl (2005) and the original papers by Akaike (1998) and Schwarz (1978).

2. The 'FIC' from Equation (2.11) with estimated $\boldsymbol{\delta}$.

3. An infeasible version of the FIC abbreviated as 'Infeas'. This information criterion is based on population quantities, especially $\boldsymbol{\delta}$ is considered to be known.

For the model averaging setup we consider:

1. 'sAIC' and 'sBIC' as smoothed counterparts of the AIC and BIC, see Burnham and Anderson (2002). To illustrate, let $A I C(m)$ denote the AIC for model $m \in \mathcal{M}$. The smoothed AIC assigns a weight of $\exp \left(-\frac{1}{2} A I C(m)\right) / \sum_{m \in \mathcal{M}} \exp \left(-\frac{1}{2} A I C(m)\right)$ to model $m$.

2. Three plug-in averages are reported. 'Plug-in' and 'Plug-in Corr.' are computed from Equations (2.14) and (2.15), where only the second average uses the bias correction on $\hat{\boldsymbol{\delta}} \hat{\boldsymbol{\delta}}^{\prime}$. The plug-in average based on known $\boldsymbol{\delta}$ is denoted 'Infeas'.

3. The 'Jackknife' model averaging procedure detailed in Hansen and Racine (2012) and Zhang et al. (2013).

\footnotetext{
${ }^{14} \mathrm{~A}$ selection of simulation results is reported here, extensive results can be found as supplemental material on the website of Econometric Reviews.

${ }^{15}$ Previous studies (e.g. Hansen (2007), Hansen (2008), Hansen and Racine (2012), Liu and Okui (2013), Zhang et al. (2013) and Liu (2015)) show the performance as a function of the population $R^{2}$. This representation is inconvenient in our dynamic setup because it is unclear when we are approaching the boundary of the stationarity region.
} 
4. The Stein combination shrinkage method used in the simulation section of Hansen (2016) is abbreviated 'SteinH'. This shrinkage method combines VAR(1) through $\operatorname{VAR}(p)$ models as well as univariate $\operatorname{AR}(1)$ through $\operatorname{AR}(p)$ models. Our DGP contains considerable interaction between the cross-sectional units so we also consider a shrinkage method abbreviated 'Stein' which only combines the VAR(1) through $\operatorname{VAR}(p)$.

\subsubsection{The simplified DGP}

Figures 2.3 and 2.4 provide the finite sample confirmation of the intuition we gained from the simplified DGP. ${ }^{16}$ The wide spread in the empirical distribution of $\widehat{F I C}_{1}$ shown in Figure 2.3(a)-(b) results indeed in a smoothed instead of binary selection between the models (see Figure 2.3(c)-(d)). The performance of the feasible FIC is therefore worse than that of the infeasible FIC that assumes $\delta$ to be known. At high $|\delta|$, we see that the probability to select the wrong model is small. The feasible FIC therefore performs similarly to its infeasible counterpart for large amounts of misspecification only.

In Figure 2.4, the four panels display results on the plug-in averages. The quantiles of the weight distribution without bias correction should be compared to those in Equation (2.19) and Figure 2.2. The results match. Figure 2.4(b) shows the quantile of the weight distribution with bias correction. As expected, this distribution is shifted towards higher weights because the upward bias of the AMSE of the model with $m=1$ is removed. For a sample size of $T=100$, we can see in Figure 2.4(c) that the plug-in averages do not perform as well as the infeasible estimator. The simulation outcome of Figure 2.4(d) at a sample size of $T=1000$ confirm that this effect does not disappear with sample size. The inconsistent estimation of $\delta$ causes the feasible weights to differ from the optimal weights.

\subsubsection{Simulation Results for an Autoregressive Model}

Further simulations are based on the following model: ${ }^{17}$

$$
y_{T, t}=0.5 y_{T, t-1}+\frac{\delta}{\sqrt{T}} y_{T, t-2}+\frac{\delta}{2 \sqrt{T}} y_{T, t-3}+u_{t}, \quad u_{t} \stackrel{\text { i.i.d. }}{\sim} \mathcal{N}(0,1) .
$$

\footnotetext{
${ }^{16}$ In this section we have rescaled the empirical MSE by the sample size to make it comparable to the asymptotic results of Figures 2.1 and 2.2, hence the label empirical asymptotic MSE.

${ }^{17}$ We show in Appendix 2.C that the simplified model with $p_{1}=p_{2}=1$, i.e. $y_{T, t}=\alpha y_{T, t-1}+\frac{\delta}{\sqrt{T}} y_{T, t-2}+u_{t}$, is special because the gradient vector has no influence on model selection and plug-in averaging. We extend the model with an additional lag to see the influence of the impulse response horizon.
} 


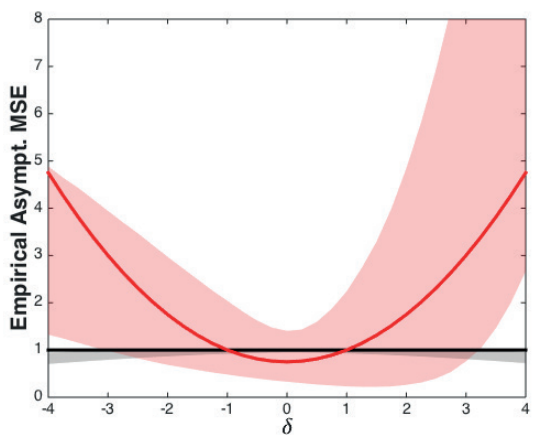

(a) $T=100$

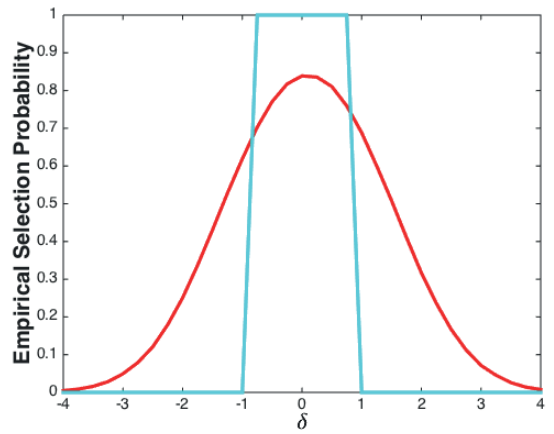

(c) $T=100$

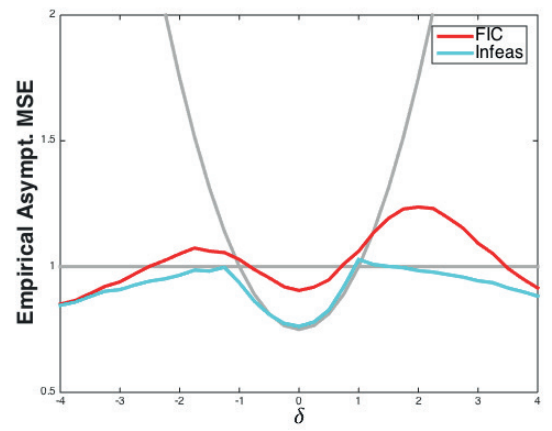

(e) $T=100$

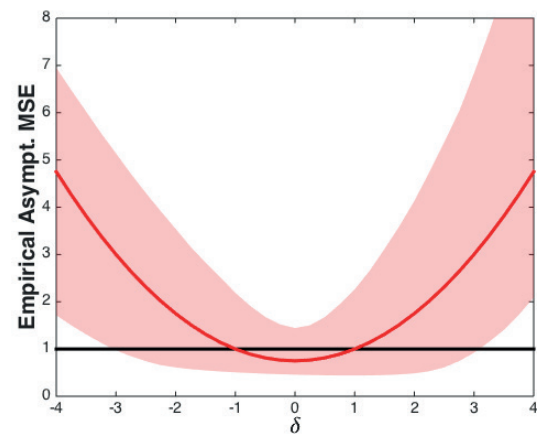

(b) $T=1000$

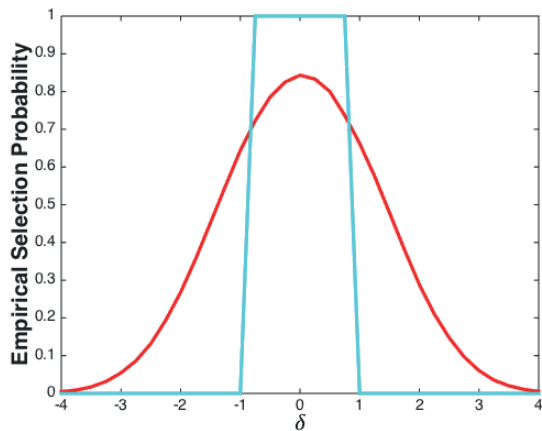

(d) $T=1000$

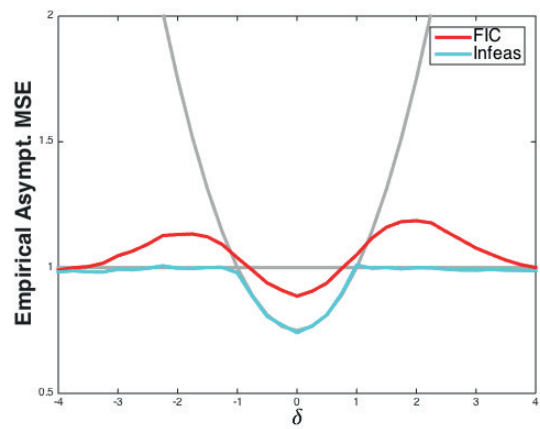

(f) $T=1000$

Figure 2.3: (a)-(b) The empirical asymptotic MSE of the models with one and two lags (red and black line, respectively). The area between the $5 \%$ and $95 \%$ empirical quantiles of $\widehat{F I C}_{1}$ and $\widehat{F I C}_{2}$ are shaded in red and grey. (c)-(d) The empirical selection probabilities of the FIC. (e)-(f) The AMSE of the models with $m=1$ and $m=2$ together with the empirical MSE of the feasible FIC (red) and infeasible FIC (cyan). This figure was obtained for $T=100$ and $T=1000$. It should be compared with the asymptotic results in Figure 2.1. 


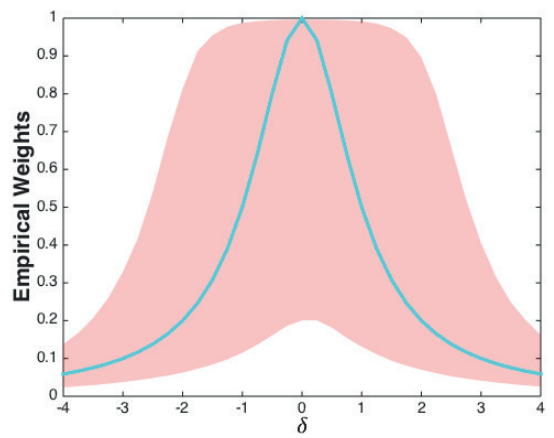

(a)

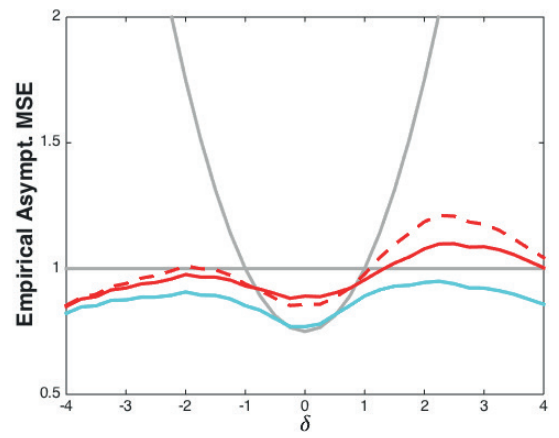

(c)

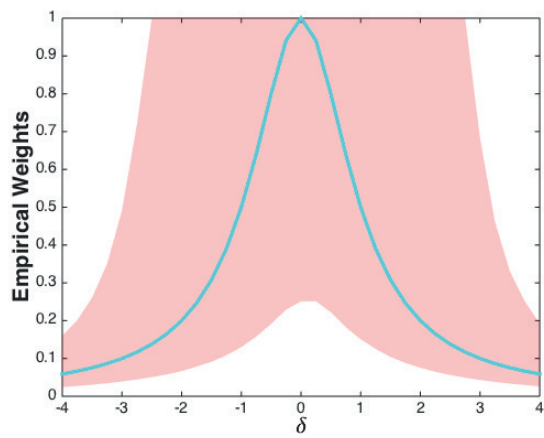

(b)

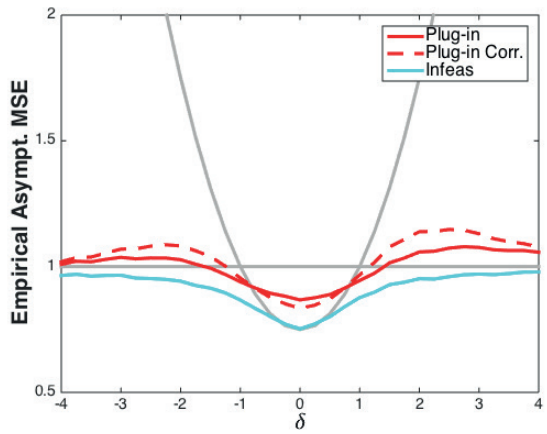

(d)

Figure 2.4: The 5\% and 95\% empirical quantiles of the weights distribution without bias correction (a) and with bias correction (b). The infeasible weights are displayed in cyan. The empirical MSE of the plug-in methods is shown in (c). The sample size for the subfigures (a)-(c) is $T=100$. Panel (d) replicates panel (c) at a sample size of $T=1000$. 
The second and third lag are local-to-zero implying that $\mathcal{M}=\{1,2,3\}$. The coefficients in front of the misspecified lags decline linearly as in Liu (2015), where $\delta$ governs the amount of misspecification.The largest modulus eigenvalue of the companion matrix is about 0.3 at $\delta=-0.2$ and increases monotonously to approximately 0.9 at the boundaries of the interval $[-4,2]$.

Remark 2.10 The AMSEs of the impulse response at horizon 1 are the same for $m=2$ and $m=3$. The plug-in weights are not unique, also see Remark 2.6.

\section{MSE Comparison}

The empirical MSE of the various selection methods are shown in Figure 2.5 for the impulse responses at horizon 1,3, and 5. Due to the strong penalty on model complexity, the BIC performs well for small amounts of misspecification, but its performance quickly deteriorates as $|\delta|$ increases. The performance of the AIC and the feasible version of the FIC are comparable for large areas of the parameter space, with neither of these methods being preferred to the other. The infeasible FIC is very frequently the preferred method.

Model averaging results are reported in Figure 2.6. The behavior of the smoothed BIC procedure is similar to that of its selection counterpart, i.e. it only performs well for small $\delta$. The same remark applies to the plug-in average with bias correction. The Jackknife, smoothed AIC, and the plug-in average without bias correction are close competitors, where the plug-in average is a better candidate for large $|\delta|$. The performance of the plugin average with known $\delta$ is best. It even performs uniformly the best at the larger sample size of $T=1000$.

What causes the superior performance of the infeasible estimators? Our simulation findings can be understood from the intuition that was gained from the simplified DGP. Panel (a) and (b) from Figure 2.7 show the empirical MSE of the three models, $m \in$ $\{1,2,3\}$, together with the AMSE of these models. The asymptotic approximation is close for $T=100$ and improves further at $T=1000$. The selection probabilities in panels (c) and (d) reveal how the infeasible estimator takes a binary decision where the chosen lag length of the AR model increases with increasing $|\delta|$. For the simplified DGP we have seen how the convergence in distribution of $\hat{\delta}$ causes smeared out selection probabilities instead of binary decisions. This effect is also observed in the graphs, even at the large sample size of $T=1000$. The panels (e) and (f) tell the same story for the plug-in weights. 
We also perform simulation where we focus on several impulse responses simultaneously, see Remarks 2.4 and 2.5. The trace is used to map the AMSE matrix to a scalar. The simulation outcomes are qualitatively similar to our results for the impulse responses at a single horizon (see Appendix 2.D).

\section{Confidence Intervals}

Confidence intervals/bands can be calculated based on Theorem 4. Simulation results are provided in Tables 2.1 and 2.2. The desired nominal coverage level was $90 \%$. Table 2.1 shows that the empirical coverage of the individual confidence intervals is always too low. At horizon 2 and 3 this under-coverage is small and decreasing with sample size. The coverage of the confidence level for horizon 6 varies strongly across $\delta$ and can be very low. It is well-established in the literature (e.g. Kilian (1998) and Kilian (2001)) that inference on impulse responses at higher horizons is inherently more difficult because of the increased nonlinearity in the parameters. This nonlinearity causes the delta method approximation to perform poorly. Figure 2.8 shows the histograms of $\sqrt{T}\left(\overline{\boldsymbol{\mu}}(\hat{\boldsymbol{w}})-\boldsymbol{\mu}\left(\boldsymbol{\theta}_{T}, \boldsymbol{\sigma}\right)\right)-\boldsymbol{D}_{\theta} \sum_{m=p_{1}}^{p} \hat{w}_{m} \hat{\boldsymbol{C}}_{m} \hat{\boldsymbol{\delta}}$ for the impulse responses at horizons 2 and 6 (corresponding to the boxed numbers in Table 2.1). Note that the confidence intervals/bands defined in Theorem 4 are based on the asymptotic normality of this expression. The sometimes severe under-coverage at horizon 6 should therefore not come as a surprise. This poor asymptotic approximation at horizon 6 also influences the empirical coverage of the confidence bands as can be seen in Table 2.2.

\subsubsection{Simulation Results for a Vector Autoregressive Model}

Further simulation results are based on a bivariate VAR with DGP

$$
\begin{aligned}
& \boldsymbol{y}_{T, t}=\left(\begin{array}{cc}
0.5 & 0 \\
0.5 & 0.5
\end{array}\right) \boldsymbol{y}_{T, t-1}+\frac{\delta}{\sqrt{T}}\left(\begin{array}{cc}
1 & 0 \\
0.5 & 1
\end{array}\right) \boldsymbol{y}_{T, t-2}+\frac{\delta}{2 \sqrt{T}}\left(\begin{array}{cc}
1 & 0 \\
0.5 & 1
\end{array}\right) \boldsymbol{y}_{T, t-3}+\boldsymbol{u}_{t}, \\
& \boldsymbol{u}_{t} \stackrel{i . i . d .}{\sim} \mathcal{N}\left(\mathbf{0}, \boldsymbol{\Sigma}_{u}\right), \text { where } \boldsymbol{\Sigma}_{u}=\left(\begin{array}{cc}
1 & 0.17 \\
0.17 & 0.33
\end{array}\right),
\end{aligned}
$$

which is similar to the VAR model used in Lütkepohl et al. (2015) for impulse response analyses. This process has the same roots as the univariate process of Equation (2.20) but with double multiplicity. The parameter $\delta$ governs the degree of misspecification. For brevity, we only report MSE results of the response of variable 1 to a structural shock in 
2 A Focused Information Criterion for Locally Misspecified Vector Autoregressive Models

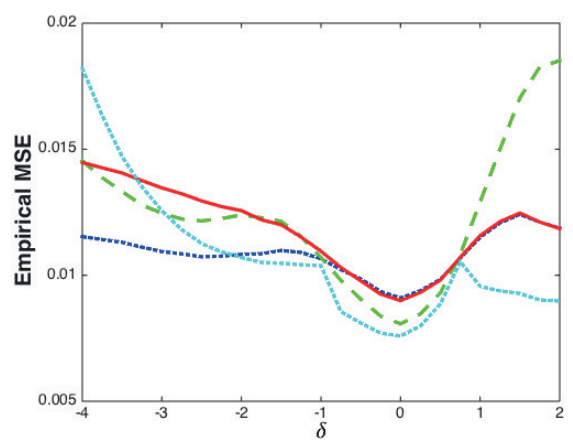

(a) $h=1, T=100$.

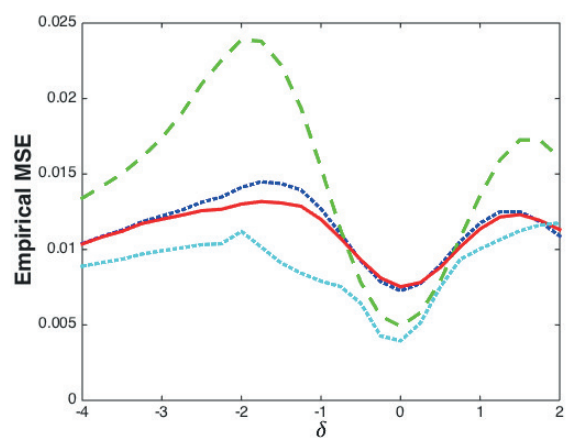

(c) $h=3, T=100$.

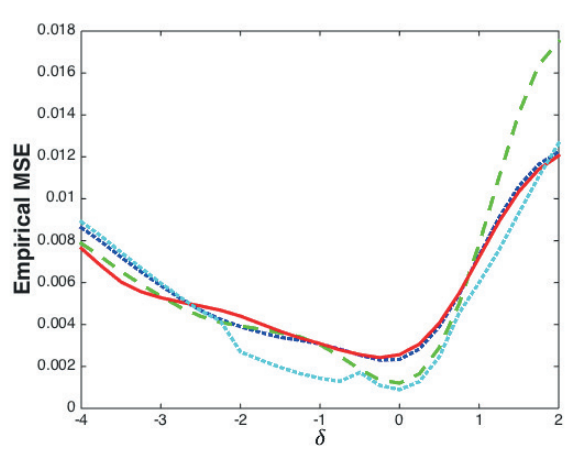

(e) $h=5, T=100$.

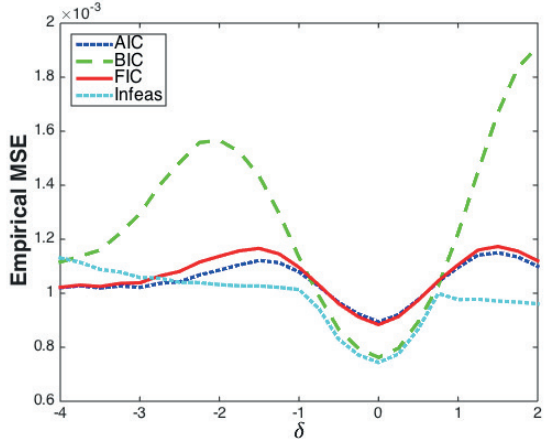

(b) $h=1, T=1000$.

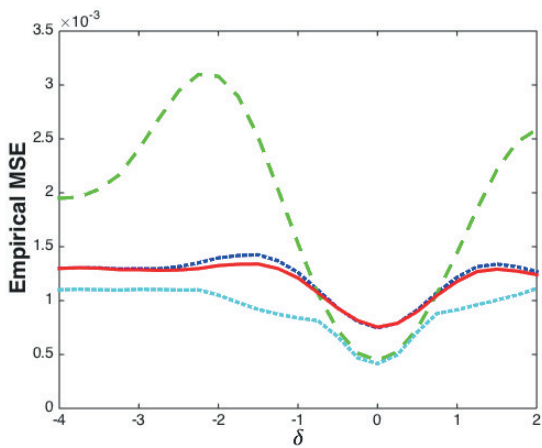

(d) $h=3, T=1000$.

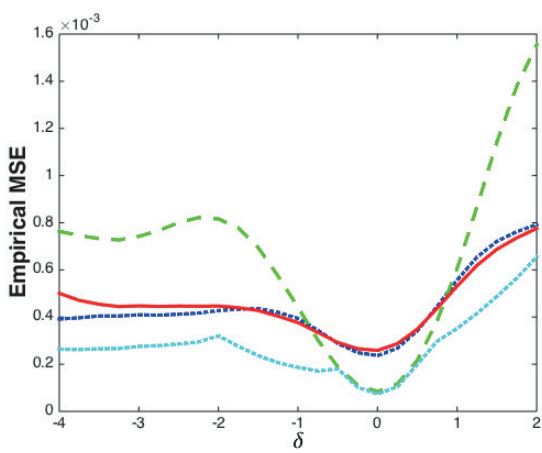

(f) $h=5, T=1000$.

Figure 2.5: The empirical MSE for model selection. The DGP is $y_{T, t}=0.5 y_{T, t-1}+\frac{\delta}{\sqrt{T}} y_{T, t-2}+$ $\frac{\delta}{2 \sqrt{T}} y_{T, t-3}+u_{t}$. 


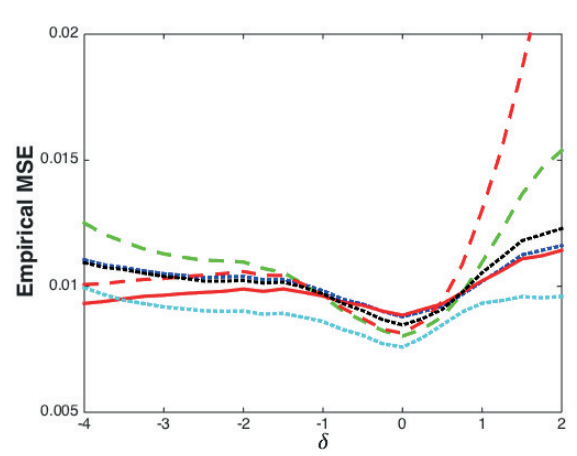

(a) $h=1, T=100$.

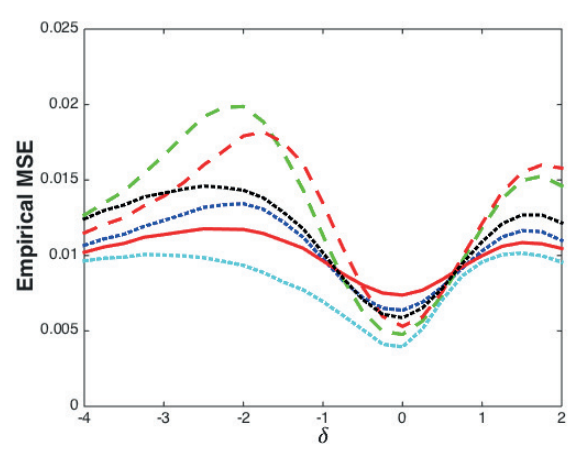

(c) $h=3, T=100$.

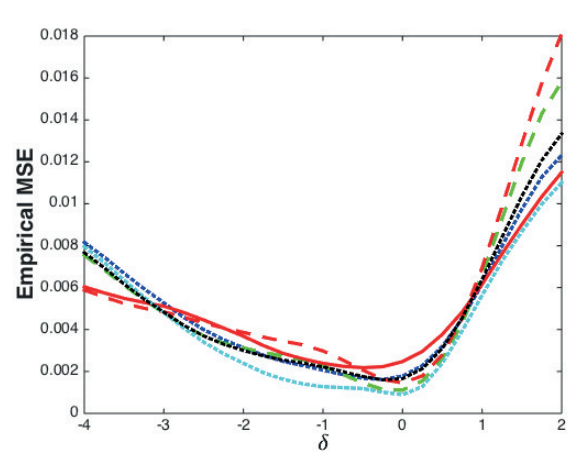

(e) $h=5, T=100$.

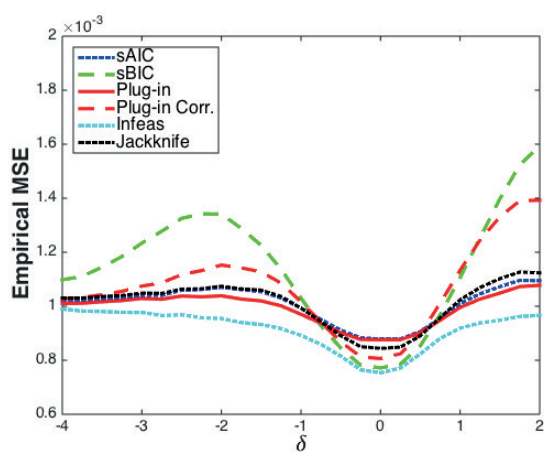

(b) $h=1, T=1000$.

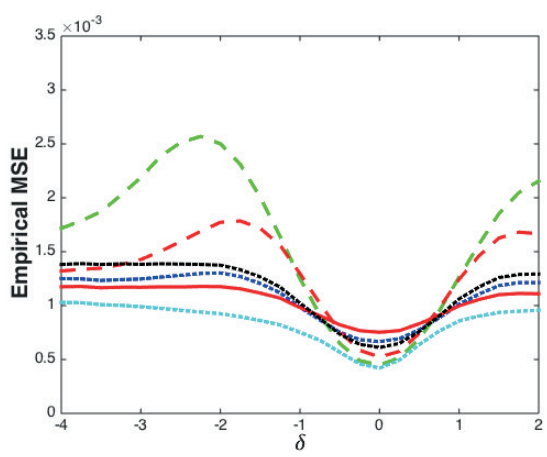

(d) $h=3, T=1000$.

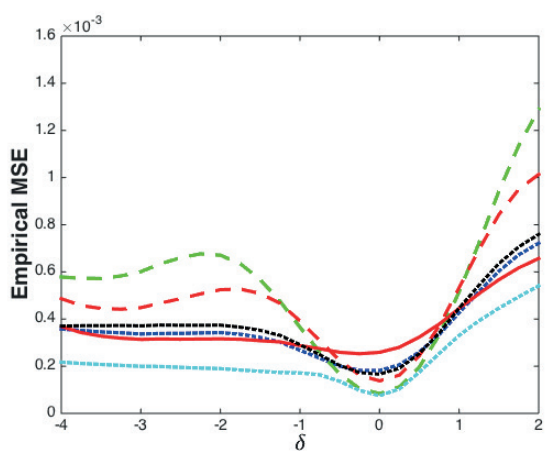

(f) $h=5, T=1000$.

Figure 2.6: The empirical MSE for model averaging. The DGP is $y_{T, t}=0.5 y_{T, t-1}+\frac{\delta}{\sqrt{T}} y_{T, t-2}+$ $\frac{\delta}{2 \sqrt{T}} y_{T, t-3}+u_{t}$. 


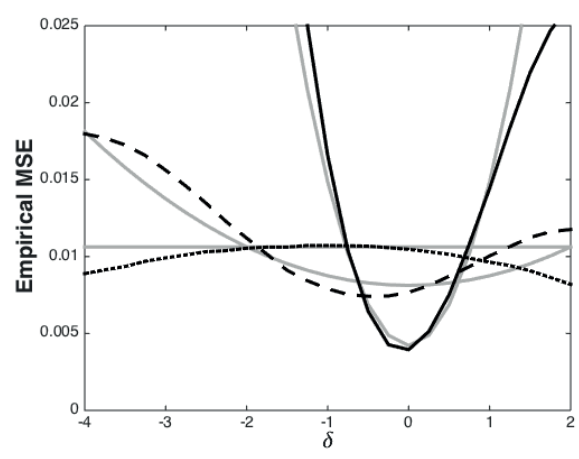

(a) $h=3, T=100$.
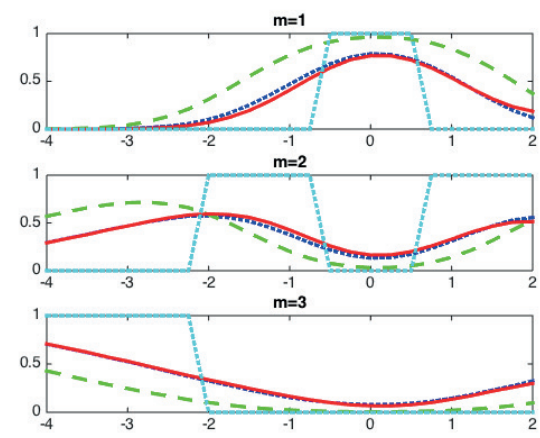

(c) $h=3, T=100$.
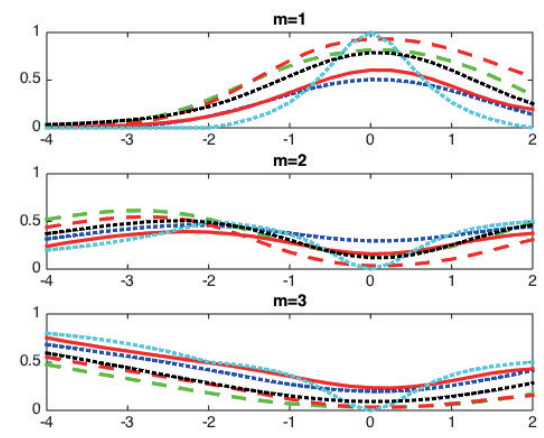

(e) $h=3, T=100$.

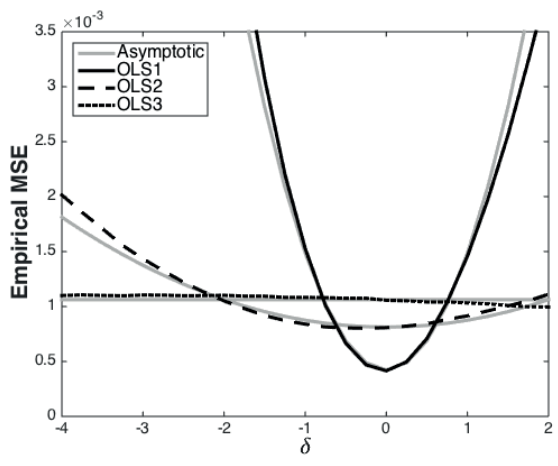

(b) $h=3, T=1000$.
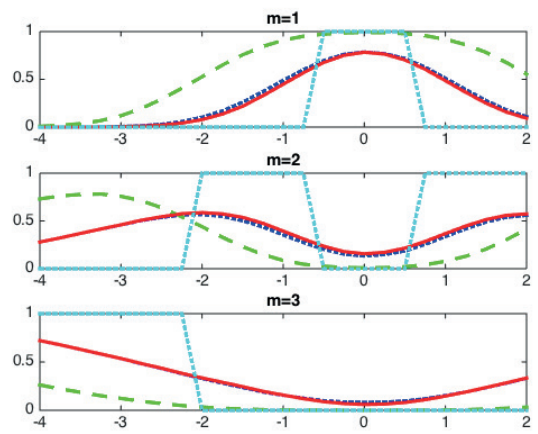

(d) $h=3, T=1000$.
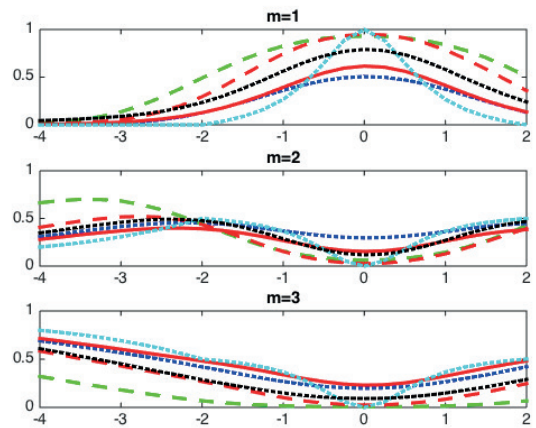

(f) $h=3, T=1000$.

Figure 2.7: (a)-(b) The empirical MSE of the OLS estimator of the model with 1 lag (OLS1), 2 lags (OLS2) and the full model with 3 lags (OLS3). Gray lines show the asymptotic MSE approximations as provided by the delta method. (c)-(d) The empirical selection probabilities (see Figure 2.5 for the appropriate legend). (e)-(f) The empirical distribution of the weights (see Figure 2.6 for the appropriate legend). The DGP is $y_{T, t}=0.5 y_{T, t-1}+\frac{\delta}{\sqrt{T}} y_{T, t-2}+\frac{\delta}{2 \sqrt{T}} y_{T, t-3}+u_{t}$ for all graphs. 


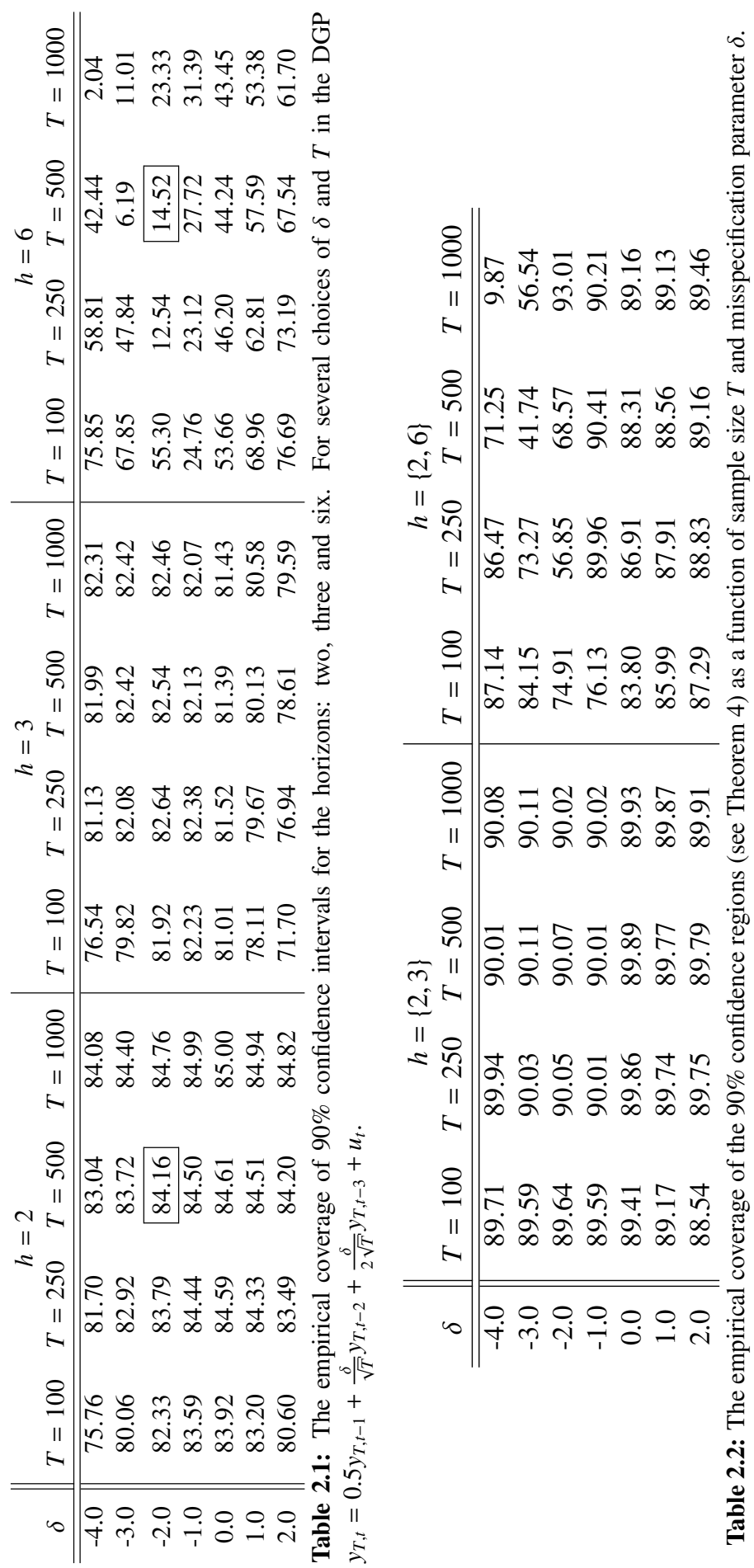




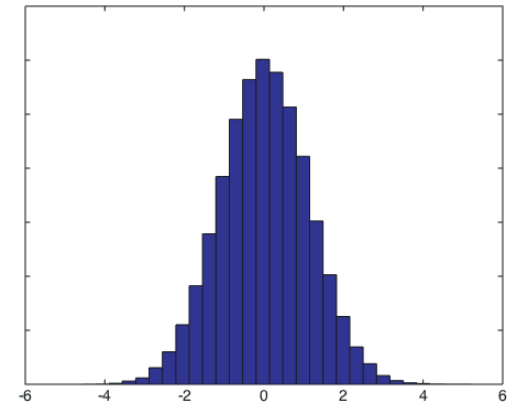

(a) $h=2, T=500$.

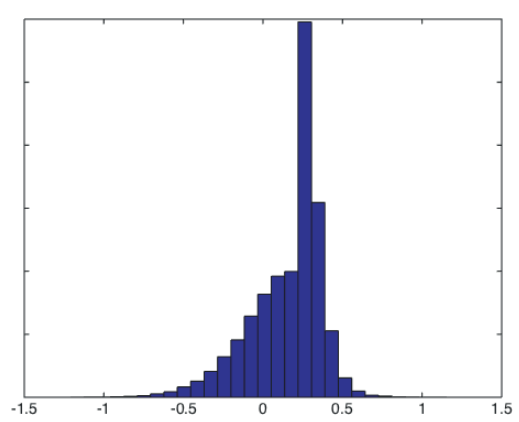

(b) $h=6, T=500$.

Figure 2.8: The confidence intervals are based on the asymptotic normality of $\sqrt{T}(\overline{\boldsymbol{\mu}}(\hat{\boldsymbol{w}})-$ $\left.\boldsymbol{\mu}\left(\boldsymbol{\theta}_{T}, \boldsymbol{\sigma}\right)\right)-\boldsymbol{D}_{\theta} \sum_{m=p_{1}}^{p} \hat{w}_{m} \hat{\boldsymbol{C}}_{m} \hat{\boldsymbol{\delta}}$ (see Theorem 4). The displayed histograms are constructed for $y_{T, t}=0.5 y_{T, t-1}+\frac{\delta}{\sqrt{T}} y_{T, t-2}+\frac{\delta}{2 \sqrt{T}} y_{T, t-3}+u_{t}$ with $\delta=-2$ and $T=500$, i.e. the boxed entries in Table 2.1. The number of Monte Carlo replications is 100, 000 .

variable 1. Figures 2.9, and 2.10 show the results for horizons 2 and $6 .{ }^{18}$ Similarly to the univariate results, none of the methods performs uniformly best. Only the infeasible methods get close to dominating all other methods for the large sample size of $T=1000$. The ragged spike for 'Infeas' in Figure 2.9(a) is caused by an abrupt binary decision to switch between models with different lag lengths. Finally, it is interesting to note that the Stein shrinkage methods perform well in comparison to the plug-in averaging procedure.

\footnotetext{
${ }^{18}$ The simulation results for all four impulse responses and horizons 1-6 can be found in the supplemental material on the site of Econometric Reviews.
} 


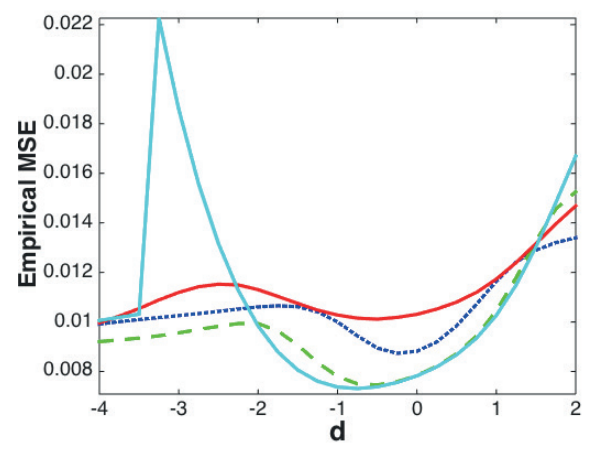

(a) $h=2$, model selection.

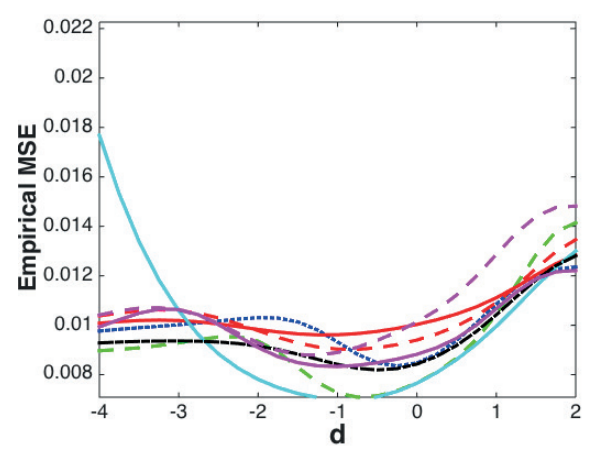

(c) $h=2$, model averaging.

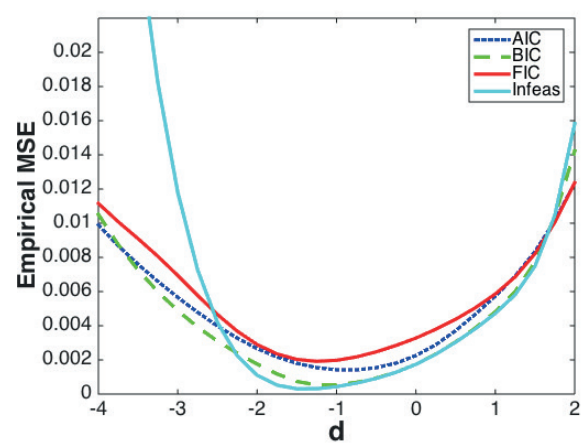

(b) $h=6$, model selection.

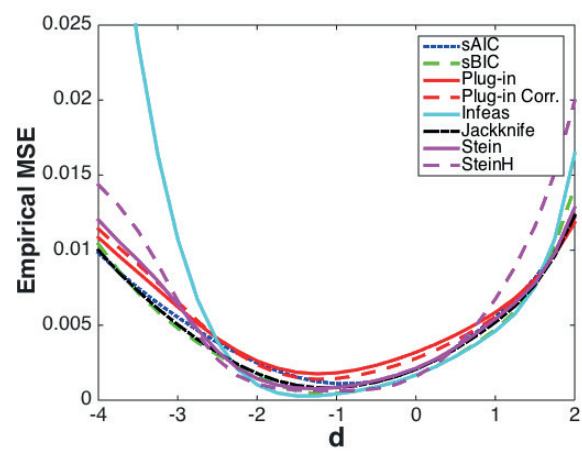

(d) $h=6$, model averaging.

Figure 2.9: The empirical MSE of the impulse response estimator for several selection and averaging methods. We have displayed the results for the response of variable 1 to a structural shock in the variable 1 for horizons 2 and 6. The DGP is given in Equation (2.21). The sample size is $T=100$. 
2 A Focused Information Criterion for Locally Misspecified Vector Autoregressive Models

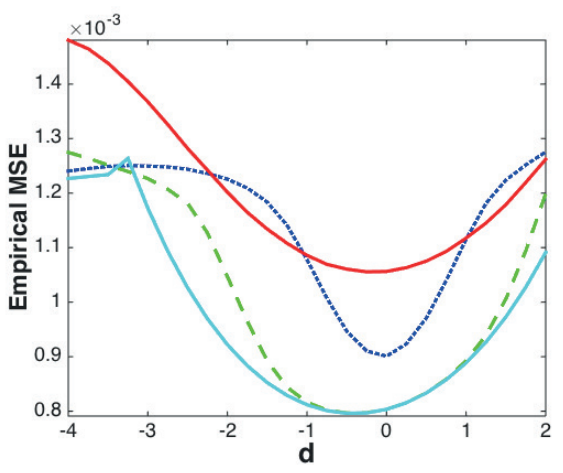

(a) $h=2$, model selection.

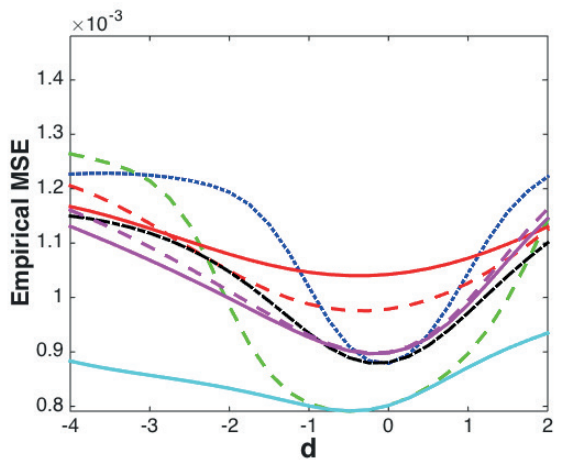

(c) $h=2$, model averaging.

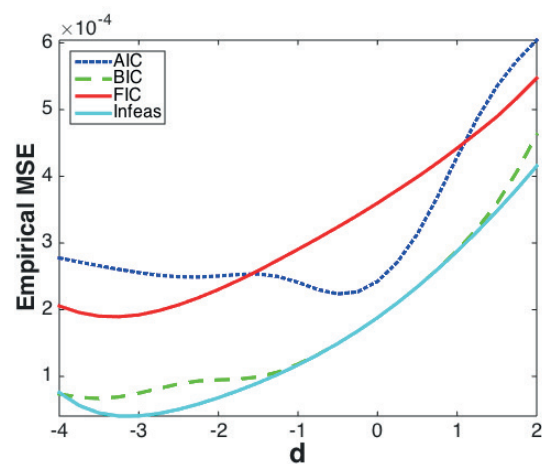

(b) $h=6$, model selection.

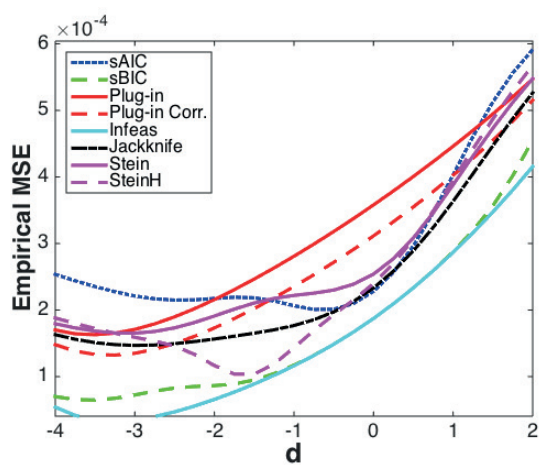

(d) $h=6$, model averaging.

Figure 2.10: Identical to Figure 2.9, but for $T=1000$. 


\subsection{Conclusion}

In this paper we studied the issue of model selection and model averaging for multivariate autoregressive processes in a locally drifting asymptotic framework. Within this drifting framework we derived the asymptotic normality of the least squares estimators. The multivariate delta method subsequently ensured that this asymptotic normality carries over to sufficiently smooth parameter transformations, e.g. impulse responses. The focused information criterion and plug-in averaging estimator were defined as the minimizers of the estimated asymptotic mean squared error of the focus parameter estimator.

We highlighted the role of the misspecification parameter $\delta$. Both Liu (2015) and DiTraglia (2016) mentioned that the feasible FIC remains random in the limit. We provided the explicit expressions for the limiting distribution of the FIC values and the elements of the weighting matrices and illustrated that the feasible estimators do not truly minimize the asymptotic mean squared error. This latter result might encourage further research into different ways to deal with the misspecification parameter. There are to the best of our knowledge two alternatives reported in the literature. The recent paper by Kitagawa and Muris (2016) adopts a mixed frequentist and Bayesian framework to alleviate the estimation of $\boldsymbol{\delta}$ in their study of model averaging in semiparametric estimation of treatment effects. Hansen (2016) similarly adopts a local-to-zero framework but minimizes a risk quantity that does not require the direct estimation of $\boldsymbol{\delta}$.

Our simulation study of univariate and multivariate autoregressive processes underlined the previous paragraph because the infeasible estimator (the estimator knowing $\boldsymbol{\delta}$ ) frequently dominated the other methods. The latter was especially the case at the larger sample size of $T=1000$. There was no clearly preferred method for feasible model selection/averaging.

A possible extension of this work is an application to forecasting. Such an extension would complement: (1) the predictive static regression setup discussed in Liu and Kuo (2016), and (2) the prediction focused model selection of autoregressive models in Claeskens et al. (2007). Forecasts for autoregressive models often start from the assumption that estimation and prediction are applied to two independent processes with the same stochastic structure. The link to this current paper is that (under this independence assumption) the asymptotic covariance matrix of the forecast is a continuous transformation of the autoregressive parameters, see section 3.5 of Lütkepohl (2005) for further details. 


\section{A Mathematical Proofs}

Proof of Theorem 1. As in Liu (2015) we first relate the parameters of the $\operatorname{VAR}(m)$ models to the parameters of the $\operatorname{VAR}(p)$. With the aid of the selection matrices we have

$$
\begin{aligned}
\hat{\boldsymbol{\Theta}}_{T, m} & =\boldsymbol{Y}_{T} \boldsymbol{Z}_{T, m}^{\prime}\left(\boldsymbol{Z}_{T, m} \boldsymbol{Z}_{T, m}^{\prime}\right)^{-1}=\left(\boldsymbol{B} \boldsymbol{L}^{\prime} \boldsymbol{Z}_{T}+\boldsymbol{C}_{T} \boldsymbol{S}_{0}^{\prime} \boldsymbol{Z}_{T}+\boldsymbol{U}\right) \boldsymbol{Z}_{T, m}^{\prime}\left(\boldsymbol{Z}_{T, m} \boldsymbol{Z}_{T, m}^{\prime}\right)^{-1} \\
& =\left(\left[\boldsymbol{B} \boldsymbol{C}_{T}\right] \boldsymbol{S}_{m} \boldsymbol{S}_{m}^{\prime} \boldsymbol{Z}_{T}+\boldsymbol{C}_{T}\left(\boldsymbol{I}_{K p_{2}}-\boldsymbol{\Pi}_{m}^{\prime} \boldsymbol{\Pi}_{m}\right) \boldsymbol{S}_{0}^{\prime} \boldsymbol{Z}_{T}+\boldsymbol{U}\right) \boldsymbol{Z}_{T, m}^{\prime}\left(\boldsymbol{Z}_{T, m} \boldsymbol{Z}_{T, m}^{\prime}\right)^{-1} \\
& =\left(\boldsymbol{\Theta}_{T, m} \boldsymbol{Z}_{T, m}+\boldsymbol{C}_{T}\left(\boldsymbol{I}_{K p_{2}}-\boldsymbol{\Pi}_{m}^{\prime} \boldsymbol{\Pi}_{m}\right) \boldsymbol{S}_{0}^{\prime} \boldsymbol{Z}_{T}+\boldsymbol{U}\right) \boldsymbol{Z}_{T, m}^{\prime}\left(\boldsymbol{Z}_{T, m} \boldsymbol{Z}_{T, m}^{\prime}\right)^{-1} \\
& =\boldsymbol{\Theta}_{T, m}+\boldsymbol{C}_{T}\left(\boldsymbol{I}_{K p_{2}}-\boldsymbol{\Pi}_{m}^{\prime} \boldsymbol{\Pi}_{m}\right) \boldsymbol{S}_{0}^{\prime} \boldsymbol{Z}_{T} \boldsymbol{Z}_{T, m}^{\prime}\left(\boldsymbol{Z}_{T, m} \boldsymbol{Z}_{T, m}^{\prime}\right)^{-1}+\boldsymbol{U} \boldsymbol{Z}_{T, m}^{\prime}\left(\boldsymbol{Z}_{T, m} \boldsymbol{Z}_{T, m}^{\prime}\right)^{-1}
\end{aligned}
$$

We rearrange terms to obtain the starting point of our analysis. Note especially how the scaling by $T^{1 / 2}$ cancels against the $T^{-1 / 2}$ decay rate of the elements in the matrix $C_{T}$. See Remark 2.1 for a discussion. An expression in terms of the fixed parameter matrix $\Delta$ remains:

$$
\begin{gathered}
\sqrt{T}\left(\hat{\Theta}_{T, m}-\Theta_{T, m}\right)=\underbrace{\sqrt{T} \boldsymbol{C}_{T}}_{\boldsymbol{\Delta}}\left(\boldsymbol{I}_{K p_{2}}-\boldsymbol{\Pi}_{m}^{\prime} \boldsymbol{\Pi}_{m}\right) \boldsymbol{S}_{0}^{\prime}\left(\frac{1}{T} \boldsymbol{Z}_{T} \boldsymbol{Z}_{T}^{\prime}\right) \boldsymbol{S}_{m}\left[\boldsymbol{S}_{m}^{\prime}\left(\frac{1}{T} \boldsymbol{Z}_{T} \boldsymbol{Z}_{T}^{\prime}\right) \boldsymbol{S}_{m}\right]^{-1} \\
+\left(\frac{1}{\sqrt{T}} \boldsymbol{U} \boldsymbol{Z}_{T}^{\prime}\right) \boldsymbol{S}_{m}\left[\boldsymbol{S}_{m}^{\prime}\left(\frac{1}{T} \boldsymbol{Z}_{T} \boldsymbol{Z}_{T}^{\prime}\right) \boldsymbol{S}_{m}\right]^{-1}
\end{gathered}
$$

If we define the vector of parameter estimates as $\hat{\boldsymbol{\theta}}_{T, m}=\operatorname{vec}\left(\hat{\boldsymbol{\Theta}}_{T, m}\right)$ and the true parameter vector $\boldsymbol{\theta}_{T, m}=\operatorname{vec}\left(\boldsymbol{\Theta}_{T, m}\right)$, then the properties of the vec operator provide

$$
\begin{aligned}
\sqrt{T}\left(\hat{\boldsymbol{\theta}}_{T, m}-\boldsymbol{\theta}_{T, m}\right)= & \left(\left[\boldsymbol{S}_{m}^{\prime}\left(\frac{1}{T} \boldsymbol{Z}_{T} \boldsymbol{Z}_{T}^{\prime}\right) \boldsymbol{S}_{m}\right]^{-1} \boldsymbol{S}_{m}^{\prime}\left(\frac{1}{T} \boldsymbol{Z}_{T} \boldsymbol{Z}_{T}^{\prime}\right) \boldsymbol{S}_{0}\left(\boldsymbol{I}_{K p_{2}}-\boldsymbol{\Pi}_{m}^{\prime} \boldsymbol{\Pi}_{m}\right) \otimes \boldsymbol{I}_{K}\right) \boldsymbol{\delta} \\
& +\left(\left[\boldsymbol{S}_{m}^{\prime}\left(\frac{1}{T} \boldsymbol{Z}_{T} \boldsymbol{Z}_{T}^{\prime}\right) \boldsymbol{S}_{m}\right]^{-1} \boldsymbol{S}_{m}^{\prime} \otimes \boldsymbol{I}_{K}\right) \frac{1}{\sqrt{T}} \sum_{t=1}^{T} \operatorname{vec}\left(\boldsymbol{u}_{t} \boldsymbol{z}_{T, t-1}^{\prime}\right),
\end{aligned}
$$

where $\boldsymbol{\delta}=\operatorname{vec}(\boldsymbol{\Delta})$. We will prove both $\operatorname{plim}_{T \rightarrow \infty} \frac{1}{T} \boldsymbol{Z}_{T} \boldsymbol{Z}_{T}^{\prime}=\operatorname{plim}_{T \rightarrow \infty} \frac{1}{T} \sum_{t=1}^{T} \boldsymbol{z}_{T, t-1} \boldsymbol{z}_{T, t-1}^{\prime}=$ $\boldsymbol{\Omega}$ and $\frac{1}{\sqrt{T}} \sum_{t=1}^{T} \operatorname{vec}\left(\boldsymbol{u}_{t} \boldsymbol{z}_{T, t-1}^{\prime}\right) \stackrel{d}{\longrightarrow} \mathrm{N}(\mathbf{0}, \boldsymbol{\Omega} \otimes \boldsymbol{\Sigma})$. These two results prove part (a) of Theorem 1, because the continuous mapping theorem implies that $\sqrt{T}\left(\hat{\boldsymbol{\theta}}_{T, m}-\boldsymbol{\theta}_{T, m}\right)=$ $\boldsymbol{A}_{m} \boldsymbol{\delta}+\left(\left[\boldsymbol{S}_{m}^{\prime} \boldsymbol{\Omega} \boldsymbol{S}_{m}\right]^{-1} \boldsymbol{S}_{m}^{\prime} \otimes \boldsymbol{I}_{K}\right) \boldsymbol{R}+o_{p}(1)$. 
We start with the proof of $\operatorname{plim}_{T \rightarrow \infty} \frac{1}{T} \boldsymbol{Z}_{T} \boldsymbol{Z}_{T}^{\prime}=\boldsymbol{\Omega}$. The process $\left\{\boldsymbol{y}_{T, t}\right\}_{t=-\infty}^{\infty}$ is stationary and ergodic for every fixed $T$ in view of Assumptions 1 and 2 (e.g. Theorem 3 on page 204 of Hannan (1970)). Define the companion matrix $\boldsymbol{A}_{T}$ and innovation vector $\boldsymbol{E}_{t}$ such that $\boldsymbol{z}_{T, t}=\boldsymbol{A}_{T} \boldsymbol{z}_{T, t-1}+\boldsymbol{E}_{t}$, i.e.

$$
\begin{aligned}
\boldsymbol{A}_{T}: & =\left[\begin{array}{cccccccccc}
\boldsymbol{B}_{1} & \boldsymbol{B}_{2} & \cdots & \boldsymbol{B}_{p_{1}-1} & \boldsymbol{B}_{p_{1}} & \frac{\boldsymbol{C}_{1}}{\sqrt{T}} & \frac{\boldsymbol{C}_{2}}{\sqrt{T}} & \ldots & \frac{\boldsymbol{C}_{p_{2}-1}}{\sqrt{T}} & \frac{\boldsymbol{C}_{p_{2}}}{\sqrt{T}} \\
\boldsymbol{I}_{K} & \mathbf{O} & \cdots & \mathbf{O} & \mathbf{O} & \mathbf{O} & \mathbf{O} & \cdots & \mathbf{O} & \mathbf{O} \\
\vdots & \vdots & & \vdots & \vdots & \vdots & \vdots & & \vdots & \vdots \\
\mathbf{O} & \mathbf{O} & \cdots & \mathbf{O} & \mathbf{O} & \mathbf{O} & \mathbf{O} & \ldots & \boldsymbol{I}_{K} & \mathbf{O}
\end{array}\right] \\
\boldsymbol{E}_{t}: & :=\left(\boldsymbol{u}_{t}^{\prime}, \mathbf{0}^{\prime}, \ldots, \mathbf{0}^{\prime}\right)^{\prime}
\end{aligned}
$$

From this extended VAR(1) form we conclude that $\boldsymbol{z}_{T, t} \boldsymbol{z}_{T, t}^{\prime}=\boldsymbol{A}_{T} \boldsymbol{z}_{T, t-1} \boldsymbol{z}_{T, t-1}^{\prime} \boldsymbol{A}_{T}^{\prime}+\boldsymbol{A} \boldsymbol{z}_{T, t-1} \boldsymbol{E}_{t}^{\prime}+$ $\boldsymbol{E}_{t-1} \boldsymbol{z}_{T, t-1}^{\prime} \boldsymbol{A}_{T}^{\prime}+\boldsymbol{E}_{t} \boldsymbol{E}_{t}^{\prime}$. Stationarity implies

$$
\begin{aligned}
& \lim _{T \rightarrow \infty}\left(\boldsymbol{I}_{K^{2} p^{2}}-\boldsymbol{A}_{T} \otimes \boldsymbol{A}_{T}\right) \operatorname{plim}_{T \rightarrow \infty} \operatorname{vec}\left(\frac{1}{T} \sum_{t=1}^{T} \boldsymbol{z}_{T, t-1} \boldsymbol{z}_{T, t-1}^{\prime}\right) \\
& =\operatorname{plim}_{T \rightarrow \infty} \operatorname{vec}\left(\frac{1}{T} \sum_{t=1}^{T} \boldsymbol{A}_{T} \boldsymbol{z}_{T, t-1} \boldsymbol{E}_{t}^{\prime}\right)+\operatorname{plim}_{T \rightarrow \infty} \operatorname{vec}\left(\frac{1}{T} \sum_{t=1}^{T} \boldsymbol{E}_{t} \boldsymbol{z}_{T, t-1}^{\prime} \boldsymbol{A}_{T}^{\prime}\right) \\
& +\operatorname{plim}_{T \rightarrow \infty} \operatorname{vec}\left(\frac{1}{T} \sum_{t=1}^{T} \boldsymbol{E}_{t} \boldsymbol{E}_{t}^{\prime}\right) .
\end{aligned}
$$

The nonrandom matrix $\boldsymbol{A}_{T}$ will converge to the matrix $\boldsymbol{A}_{\infty}$ for large $T . \boldsymbol{A}_{\infty}$ is thus obtained from $\boldsymbol{A}_{T}$ by replacing the ratios $C_{i} / \sqrt{T}$ with zero matrices for $i \in\left\{1,2, \ldots, p_{2}\right\}$. Note that the eigenvalues of $\boldsymbol{A}_{\infty}$ coincide with the roots of the matrix polynomial $\boldsymbol{B}_{\infty}(z)$ augmented with $K p_{2}$ additional zero eigenvalues. Assumption 3 guarantees that the ma$\operatorname{trix} \boldsymbol{I}_{p^{2}}-\boldsymbol{A}_{\infty} \otimes \boldsymbol{A}_{\infty}$ is invertible.

Subsequently we consider the RHS of Equation (2.26). Let $y_{T, t-j, k}$ and $u_{t, k}$ denote the $k^{\prime}$ th component of $\boldsymbol{y}_{T, t-j}$ and $\boldsymbol{u}_{t}$, respectively. If we can show that $\frac{1}{T} \sum_{t=1}^{T} y_{T, t-j, k} u_{t, l} \stackrel{p}{\longrightarrow} 0$ for all $j \in\{1,2, \ldots, p\}$ and $k, l \in\{1,2, \ldots, K\}$, then the first two terms in the RHS of Equation (2.26) are $o_{p}(1)$. To prove this, we define the array $X_{T, t}^{j k l}=y_{T, t-j, k} u_{t, l} / T$ and the norming $c_{T}=1 / T . X_{T, t}^{j k l}$ is a martingale difference (m.d.) array with respect to the filtration $\mathcal{F}_{t}=\sigma\left(\boldsymbol{u}_{s},-\infty<s \leq t\right)$ and $\mathrm{E}\left|X_{T, t}^{j k l} / c_{T}\right|^{4}=\mathrm{E}\left|y_{T, t-j, k} u_{t, l}\right|^{4}$ is finite in view of Assumption 1. Result 12.10 from Davidson (1994) implies that $\left|X_{T, t}^{j k l}\right|^{2}$ is uniformly integrable and Result 19.7 from the same reference gives $\frac{1}{T} \sum_{t=1}^{T} y_{T, t-j, k} u_{t} \stackrel{L_{2}}{\rightarrow} 0$. The result for the first two 
terms follows. The third term in the RHS of Equation (2.26) is a sample mean of an i.i.d. sequence. Khinchine's Theorem gives the probability limit. Combining all the results, we conclude that

$$
\operatorname{vec}(\boldsymbol{\Omega}):=\operatorname{plim}_{T \rightarrow \infty} \operatorname{vec}\left(\frac{1}{T} \sum_{t=1}^{T} \boldsymbol{z}_{T, t-1} \boldsymbol{z}_{T, t-1}^{\prime}\right)=\left(\boldsymbol{I}_{K^{2} p^{2}}-\boldsymbol{A}_{\infty} \otimes \boldsymbol{A}_{\infty}\right)^{-1} \operatorname{vec}\left(\boldsymbol{\Sigma}^{*}\right)+o_{p}(1)
$$

where $\Sigma^{*}=e \Sigma e^{\prime}$ and $e$ is the $(K p \times K)$ matrix composed of the first $K$ column of $I_{K p}$. This shows that $\operatorname{plim}_{T \rightarrow \infty} \frac{1}{T} \boldsymbol{Z}_{T} \boldsymbol{Z}_{T}^{\prime}=\boldsymbol{\Omega}$. Ergodicity for every $T$ also provides $\left(\boldsymbol{I}_{K^{2} p^{2}}\right.$ $\left.\boldsymbol{A}_{T} \otimes \boldsymbol{A}_{T}\right) \operatorname{vec}\left(\mathrm{E}\left(\boldsymbol{z}_{T, t-1} \boldsymbol{z}_{T, t-1}^{\prime}\right)\right)=\operatorname{vec}(\boldsymbol{\Sigma})$ and hence $\boldsymbol{\Omega}=\lim _{T \rightarrow \infty} \mathrm{E}\left(\boldsymbol{z}_{T, t-1} \boldsymbol{z}_{T, t-1}^{\prime}\right)$ because $\boldsymbol{A}_{T} \rightarrow \boldsymbol{A}_{\infty}$.

We rely on the Cramer-Wold theorem (e.g. Result 25.5 from Davidson (1994)) to prove the convergence of $\frac{1}{\sqrt{T}} \sum_{t=1}^{T} \operatorname{vec}\left(\boldsymbol{u}_{t} \boldsymbol{z}_{T, t-1}^{\prime}\right)$ to $\boldsymbol{R} \sim \mathrm{N}(\mathbf{0}, \boldsymbol{\Omega} \otimes \boldsymbol{\Sigma})$. Let $\boldsymbol{\xi}$ denote a fixed $\left(K^{2} p \times 1\right)$ vector. $X_{T, t}^{*}=\boldsymbol{\xi}^{\prime} \operatorname{vec}\left(\boldsymbol{u}_{t} z_{T, t-1}^{\prime}\right)$ is a m.d. array with respect to $\mathcal{F}_{t}$. We note that $\sigma_{T t}^{2}=\mathrm{E}\left(X_{T, t}^{* 2} \mid \mathcal{F}_{t-1}\right)=\mathrm{E}\left(\boldsymbol{\xi}^{\prime} \operatorname{vec}\left(\boldsymbol{u}_{T} \boldsymbol{z}_{T, t-1}^{\prime}\right) \operatorname{vec}\left(\boldsymbol{u}_{T} \boldsymbol{z}_{T, t-1}^{\prime}\right)^{\prime} \boldsymbol{\xi} \mid \mathcal{F}_{t-1}\right)=\mathrm{E}\left(\boldsymbol{\xi}^{\prime}\left(\left(\boldsymbol{z}_{T, t-1} \boldsymbol{z}_{T, t-1}^{\prime}\right) \otimes\right.\right.$ $\left.\left.\left(\boldsymbol{u}_{t} \boldsymbol{u}_{t}\right)\right) \boldsymbol{\xi} \mid \mathcal{F}_{t-1}\right)=\boldsymbol{\xi}^{\prime}\left(\left(\boldsymbol{z}_{T, t-1} \boldsymbol{z}_{T, t-1}^{\prime}\right) \otimes \boldsymbol{\Sigma}\right) \boldsymbol{\xi}$ and that $X_{T, t}^{* 2}$ is square integrable by Assumption 1. From $s_{T}^{2}=\sum_{t=1}^{T} \mathrm{E}\left(X_{T, t}^{* 2}\right)=\boldsymbol{\xi}^{\prime}\left(\mathrm{E}\left(\boldsymbol{z}_{T, t-1} \boldsymbol{z}_{T, t-1}^{\prime}\right) \otimes \boldsymbol{\Sigma}\right) \boldsymbol{\xi}$ we have

$$
\sup _{T} \frac{T}{s_{T}^{2}}=\sup _{T} \frac{1}{\xi^{\prime}\left(\mathrm{E}\left(z_{T, t-1} z_{T, t-1}^{\prime}\right) \otimes \Sigma\right) \xi}<\infty \text {. }
$$

Equation (2.28) holds because the quadratic form in the denominator cannot be zero as both $\boldsymbol{\Sigma}$ and $\mathrm{E}\left(\boldsymbol{z}_{T, t-1} \boldsymbol{z}_{T, t-1}^{\prime}\right)$ are positive definite matrices. The positive definiteness of $\boldsymbol{\Sigma}$ is part of Assumption 4. For finite $T, \mathrm{E}\left(\boldsymbol{z}_{T, t-1} z_{T, t-1}^{\prime}\right)$ cannot be positive semidefinite because this would imply the existence of a $\boldsymbol{\kappa} \neq \mathbf{0}$ such that $\boldsymbol{\kappa}^{\prime} \mathrm{E}\left(\boldsymbol{z}_{T, t-1} \boldsymbol{z}_{T, t-1}^{\prime}\right) \boldsymbol{\kappa}=\mathrm{E}\left(\boldsymbol{\kappa}^{\prime} \boldsymbol{z}_{T, t-1}\right)^{2}=0$ and at least one component of $z_{T, t-1}$ is zero for all $t$. Also it cannot approach a positive semidefinite matrix due to convergence to $\Omega$. A generalization of Result 24.4 from Davidson (1994) to martingale difference arrays shows that

$$
\frac{\frac{1}{\sqrt{T}} \sum_{t=1}^{T} \boldsymbol{\xi}^{\prime} \operatorname{vec}\left(\boldsymbol{u}_{t} \boldsymbol{z}_{T, t-1}^{\prime}\right)}{\sqrt{\boldsymbol{\xi}^{\prime}\left(\mathrm{E}\left(\boldsymbol{z}_{T, t-1} \boldsymbol{z}_{T, t-1}^{\prime}\right) \otimes \Sigma\right) \boldsymbol{\xi}}} \stackrel{d}{\rightarrow} \mathrm{N}(0,1)
$$

The expression under the square root is asymptotically equivalent to $\boldsymbol{\xi}^{\prime}(\boldsymbol{\Omega} \otimes \boldsymbol{\Sigma}) \boldsymbol{\xi}$. The second result, $\frac{1}{\sqrt{T}} \sum_{t=1}^{T} \operatorname{vec}\left(\boldsymbol{u}_{t} \boldsymbol{z}_{T, t-1}^{\prime}\right) \stackrel{d}{\longrightarrow} \mathrm{N}(\mathbf{0}, \boldsymbol{\Omega} \otimes \boldsymbol{\Sigma})$, follows because $\boldsymbol{\xi}$ is arbitrary. The proof of part (a) is complete. 
Part (b) of Theorem 1 is a joint convergence result with the estimator for the covariance matrix. For any model with $m \in\left\{p_{1}, p_{1}+1, \ldots, p\right\}$ lags we define the residual matrix (residuals are stacked columnwise) by $\hat{\boldsymbol{U}}_{T, t}^{m}=\boldsymbol{Y}_{T}-\hat{\boldsymbol{B}}_{T, m} \boldsymbol{Z}_{T, m}$. The estimated covariance matrix based on the residuals from the model with $m$ lags satisfies

$$
\begin{aligned}
\hat{\Sigma}_{u}^{m}=\frac{1}{T}\left(\boldsymbol{Y}_{T}-\hat{\boldsymbol{\Theta}}_{T, m} \boldsymbol{Z}_{T, m}\right)\left(\boldsymbol{Y}_{T}-\hat{\boldsymbol{\Theta}}_{T, m} \boldsymbol{Z}_{T, m}\right)^{\prime} \\
=\frac{1}{T}\left[\left(\boldsymbol{\Theta}_{T, m}-\hat{\boldsymbol{\Theta}}_{T, m}\right) \boldsymbol{S}_{m}^{\prime} \boldsymbol{Z}_{T}+\boldsymbol{C}_{T}\left(\boldsymbol{I}_{K p_{2}}-\boldsymbol{\Pi}_{m}^{\prime} \boldsymbol{\Pi}_{m}\right) \boldsymbol{S}_{0}^{\prime} \boldsymbol{Z}_{T}+\boldsymbol{U}\right] \\
\quad\left[\left(\boldsymbol{\Theta}_{T, m}-\hat{\boldsymbol{\Theta}}_{T, m}\right) \boldsymbol{S}_{m}^{\prime} \boldsymbol{Z}_{T}+\boldsymbol{C}_{T}\left(\boldsymbol{I}_{K p_{2}}-\boldsymbol{\Pi}_{m}^{\prime} \boldsymbol{\Pi}_{m}\right) \boldsymbol{S}_{0}^{\prime} \boldsymbol{Z}_{T}+\boldsymbol{U}\right]^{\prime} \\
=\left(\boldsymbol{\Theta}_{T, m}-\hat{\boldsymbol{\Theta}}_{T, m}\right) \boldsymbol{S}_{m}^{\prime}\left(\frac{\boldsymbol{Z}_{T} \boldsymbol{Z}_{T}^{\prime}}{T}\right) \boldsymbol{S}_{m}\left(\boldsymbol{\Theta}_{T, m}-\hat{\boldsymbol{\Theta}}_{T, m}\right)^{\prime} \\
+\boldsymbol{C}_{T}\left(\boldsymbol{I}_{K p_{2}}-\boldsymbol{\Pi}_{m}^{\prime} \boldsymbol{\Pi}_{m}\right) \boldsymbol{S}_{0}^{\prime}\left(\frac{\boldsymbol{Z}_{T} \boldsymbol{Z}_{T}^{\prime}}{T}\right) \boldsymbol{S}_{0}\left(\boldsymbol{I}_{K p_{2}}-\boldsymbol{\Pi}_{m}^{\prime} \boldsymbol{\Pi}_{m}\right)^{\prime} \boldsymbol{C}_{T}^{\prime} \\
+\left(\boldsymbol{\Theta}_{T, m}-\hat{\boldsymbol{\Theta}}_{T, m}\right) \boldsymbol{S}_{m}^{\prime}\left(\frac{\boldsymbol{Z}_{T} \boldsymbol{Z}_{T}^{\prime}}{T}\right) \boldsymbol{S}_{0}\left(\boldsymbol{I}_{K p_{2}}-\boldsymbol{\Pi}_{m}^{\prime} \boldsymbol{\Pi}_{m}\right)^{\prime} \boldsymbol{C}_{T}^{\prime} \\
+\left(\boldsymbol{\Theta}_{T, m}-\hat{\boldsymbol{\Theta}}_{T, m}\right) \boldsymbol{S}_{m}^{\prime}\left(\frac{\boldsymbol{Z}_{T} \boldsymbol{U}^{\prime}}{T}\right) \\
+\boldsymbol{C}_{T}\left(\boldsymbol{I}_{K p_{2}}-\boldsymbol{\Pi}_{m}^{\prime} \boldsymbol{\Pi}_{m}\right) \boldsymbol{S}_{0}^{\prime}\left(\frac{\boldsymbol{Z}_{T} \boldsymbol{Z}_{T}^{\prime}}{T}\right) \boldsymbol{S}_{m}\left(\boldsymbol{\Theta}_{T, m}-\hat{\boldsymbol{\Theta}}_{T, m}\right)^{\prime} \\
+\boldsymbol{C}_{T}\left(\boldsymbol{I}_{K p_{2}}-\boldsymbol{\Pi}_{m}^{\prime} \boldsymbol{\Pi}_{m}\right) \boldsymbol{S}_{0}^{\prime}\left(\frac{\boldsymbol{Z}_{T} \boldsymbol{U}^{\prime}}{T}\right)+\left(\frac{\boldsymbol{U}_{T}^{\prime}}{T}\right) \boldsymbol{S}_{m}\left(\boldsymbol{\Theta}_{T, m}-\hat{\boldsymbol{\Theta}}_{T, m}\right) \\
+\left(\frac{\boldsymbol{U} \boldsymbol{Z}_{T}}{T}\right) \boldsymbol{S}_{0}\left(\boldsymbol{I}_{K p_{2}}-\boldsymbol{\Pi}_{m}^{\prime} \boldsymbol{\Pi}_{m}\right)^{\prime} \boldsymbol{C}_{T}^{\prime}+\frac{1}{T} \boldsymbol{U} \boldsymbol{U}^{\prime} .
\end{aligned}
$$

The stochastic orders of the various terms in Equation (2.30) are known from previous results. We have $\boldsymbol{\Theta}_{T, m}-\hat{\boldsymbol{\Theta}}_{T, m}=O_{p}\left(T^{-1 / 2}\right), \boldsymbol{Z}_{T} \boldsymbol{Z}_{T}^{\prime} / T \stackrel{p}{\longrightarrow} \Omega, \boldsymbol{C}_{T}=O\left(T^{-1 / 2}\right)$ and $\boldsymbol{Z}_{T} \boldsymbol{U}^{\prime} / T=O_{p}\left(T^{-1 / 2}\right)$ by Equation (2.29). We conclude that $\hat{\boldsymbol{\Sigma}}_{u}^{m}=\frac{1}{T} \boldsymbol{U} \boldsymbol{U}^{\prime}+o_{P}(1)$. Every covariance estimator (every in the sense of for all $m \in \mathcal{M}$ ) has therefore the same asymptotic distribution as the covariance estimator based on the true innovations.

Joint asymptotic normality of the parameter estimates and the covariance estimator can be obtained along the lines of the proof of Proposition 11.2 of Hamilton (1994). That is, we define

$$
\boldsymbol{\lambda}_{t}=\operatorname{vech}\left[\begin{array}{cccc}
u_{1 t}^{2}-\sigma_{11} & u_{1 t} u_{2 t}-\sigma_{12} & \ldots & u_{1 t} u_{K t}-\sigma_{1 K} \\
u_{2 t} u_{1 t}-\sigma_{21} & u_{2 t}^{2}-\sigma_{22} & \ldots & u_{2 t} u_{K t}-\sigma_{2 K} \\
\vdots & \vdots & \ddots & \vdots \\
u_{K t} u_{1 t}-\sigma_{K 1} & u_{K t} u_{2 t}-\sigma_{K 2} & \ldots & u_{K t}^{2}-\sigma_{K K}
\end{array}\right]
$$


The sequence $\left\{\boldsymbol{\lambda}_{t}\right\}$ is i.i.d. and thus also a martingale difference sequence. One can apply the Cramer-Wold Theorem to the extended martingale difference vector $\left(\operatorname{vec}\left(\boldsymbol{u}_{t} \boldsymbol{z}_{T, t-1}^{\prime}\right)^{\prime}, \boldsymbol{\lambda}_{t}^{\prime}\right)^{\prime}$ to show

$$
\left[\begin{array}{c}
(1 \sqrt{T}) \sum_{t=1}^{T} \operatorname{vec}\left(\boldsymbol{u}_{t} \boldsymbol{z}_{T, t-1}^{\prime}\right) \\
(1 \sqrt{T}) \sum_{t=1}^{T} \boldsymbol{\lambda}_{t}
\end{array}\right] \stackrel{d}{\longrightarrow} \mathrm{N}\left(\left[\begin{array}{l}
\mathbf{0} \\
\mathbf{0}
\end{array}\right],\left[\begin{array}{ll}
\boldsymbol{\Xi}_{11} & \boldsymbol{\Xi}_{12} \\
\boldsymbol{\Xi}_{21} & \boldsymbol{\Xi}_{22}
\end{array}\right]\right)
$$

We already know that $\Xi_{11}=\Omega \otimes \Sigma$. The elements in the covariance matrix $\boldsymbol{\Xi}_{12}$ take the form $\lim _{T \rightarrow \infty} \mathrm{E}\left(u_{k_{1} t} y_{T, t-j, k_{2}}\left(u_{k_{3} t} u_{k_{4} t}-\sigma_{k_{3} k_{4}}\right)\right)$. They are zero because $\lim _{T \rightarrow \infty} \mathrm{E}\left(y_{T, t-j, k_{2}}\right)=$ 0 . Finally, $\boldsymbol{\Xi}_{22}=\mathrm{E}\left(\boldsymbol{\lambda}_{t} \boldsymbol{\lambda}_{t}^{\prime}\right)$. The typical elements are $\mathrm{E}\left(\left(u_{i t} u_{j t}-\sigma_{i j}\right)\left(u_{l t} u_{m t}-\sigma_{l m}\right)\right)$.

Define two independent random vectors: $\boldsymbol{R} \sim \mathrm{N}(\mathbf{0}, \boldsymbol{\Omega} \otimes \boldsymbol{\Sigma})$ and $\boldsymbol{S} \sim \mathrm{N}\left(\mathbf{0}, \boldsymbol{\Xi}_{22}\right)$. We will proof the claim in part (c) of Theorem 1 for the case of three different models indexed by $m_{1}, m_{2}, m_{3} \in \mathcal{M}$. The proof is immediate, since

$$
\left[\begin{array}{c}
\sqrt{T}\left(\hat{\boldsymbol{\theta}}_{T, m_{1}}-\boldsymbol{\theta}_{T, m_{1}}\right) \\
\sqrt{T}\left(\hat{\boldsymbol{\theta}}_{T, m_{2}}-\boldsymbol{\theta}_{T, m_{2}}\right) \\
\sqrt{T} \operatorname{vech}\left(\hat{\boldsymbol{\Sigma}}_{u}^{m_{3}}-\boldsymbol{\Sigma}_{u}\right)
\end{array}\right] \stackrel{d}{\longrightarrow}\left[\begin{array}{c}
\boldsymbol{A}_{m_{1}} \\
\boldsymbol{A}_{m_{2}} \\
\mathbf{O}
\end{array}\right] \boldsymbol{\delta}+\left[\begin{array}{cc}
\left(\left[\boldsymbol{S}_{m_{1}}^{\prime} \boldsymbol{\Omega S}_{m_{1}}\right]^{-1} \boldsymbol{S}_{m_{1}}^{\prime} \otimes \boldsymbol{I}_{K}\right) & \mathbf{O} \\
\left(\left[\boldsymbol{S}_{m_{2}}^{\prime} \boldsymbol{\Omega} \boldsymbol{S}_{m_{2}}\right]^{-1} \boldsymbol{S}_{m_{2}}^{\prime} \otimes \boldsymbol{I}_{K}\right) & \mathbf{O} \\
\mathbf{O} & \boldsymbol{I}_{K(K+1) / 2}
\end{array}\right]\left[\begin{array}{c}
\boldsymbol{R} \\
\boldsymbol{S}
\end{array}\right]
$$

Proof of the Auxiliary Result. The proof uses mathematical induction so let us compare the impulse responses of the $\operatorname{VAR}(p)$ and $\operatorname{VAR}(p+1)$ models. For $p=0$ we are comparing a white noise model with a $\operatorname{VAR}(1)$ with coefficient matrix $\boldsymbol{A}$. The impulse responses at horizon $h$ (the case $h=1$ is trivial so we focus on $h>1$ ) for these models are $\mathbf{O}_{K \times K}$ and $\boldsymbol{A}^{h}$, respectively. The base case $p=0$ holds.

We start with inductive step by defining the companion matrix of the $\operatorname{VAR}(p+1)$,

$$
\boldsymbol{F}_{(p+1)}=\left[\begin{array}{ccccc}
\boldsymbol{A}_{1} & \boldsymbol{A}_{2} & \ldots & \boldsymbol{A}_{p} & \boldsymbol{A}_{p+1} \\
\boldsymbol{I}_{K} & \mathbf{O} & \ldots & \mathbf{O} & \mathbf{O} \\
\mathbf{O} & \boldsymbol{I}_{K} & \ldots & \mathbf{O} & \mathbf{O} \\
\vdots & \vdots & \ddots & \vdots & \vdots \\
\mathbf{O} & \mathbf{O} & \ldots & \boldsymbol{I}_{K} & \mathbf{O}
\end{array}\right]
$$

This companion matrix is $(K(p+1) \times K(p+1))$. For an arbitrary matrix of this size, say $\boldsymbol{A}$, we will introduce the notation $[\boldsymbol{A}]^{i j}$ to denote its $(i, j)$ 'th block of dimension $(K \times K)$. In this notation, the impulse response at horizon $h$ for the $\operatorname{VAR}(p+1)$ is simply $\left[\left(\boldsymbol{F}_{p+1}\right)^{h}\right]^{11}$. 
Setting $\boldsymbol{A}_{p+1}=\mathbf{O}$ provides $K$ zero columns, and hence

$$
\begin{aligned}
& {\left[\left(\boldsymbol{F}_{p+1}\right)^{h}\right]^{11}} \\
& =\sum_{j_{1}=1}^{p+1} \cdots \sum_{j_{h-1}=1}^{p+1}\left[\boldsymbol{F}_{p+1}\right]^{1 j_{1}}\left[\boldsymbol{F}_{p+1}\right]^{j_{1} j_{2}} \cdots\left[\boldsymbol{F}_{p+1}\right]^{j_{h-1} 1} \\
& =\sum_{j_{1}=1}^{p}\left[\boldsymbol{F}_{p+1}\right]^{1 j_{1}}\left(\sum_{j_{2}=1}^{p+1} \cdots \sum_{j_{h-1}=1}^{p+1}\left[\boldsymbol{F}_{p+1}\right]^{j_{1} j_{2}} \cdots\left[\boldsymbol{F}_{p+1}\right]^{j_{h-1} 1}\right) \\
& =\ldots=\sum_{j_{1}=1}^{p} \ldots \sum_{j_{h-1}=1}^{p}\left[\boldsymbol{F}_{p+1}\right]^{1 j_{1}}\left[\boldsymbol{F}_{p+1}\right]^{j_{1} j_{2}} \cdots\left[\boldsymbol{F}_{p+1}\right]^{j_{h-1} 1}=\left[\left(\boldsymbol{F}_{p}\right)^{h}\right]^{11} .
\end{aligned}
$$

where $\boldsymbol{F}_{p}$ is the companion matrix related to the $\operatorname{VAR}(p)$. The inductive step is also complete and the results follows by mathematical induction.

Proof of Theorem 2. We rewrite the expression in Theorem 2 as

$$
\begin{aligned}
& \sqrt{T}\left(\boldsymbol{\mu}\left(\hat{\boldsymbol{\theta}}_{T, m}, \mathbf{0}_{K^{2}(p-m)}, \hat{\boldsymbol{\sigma}}\right)-\boldsymbol{\mu}\left(\boldsymbol{\theta}_{T, p}, \boldsymbol{\sigma}\right)\right)= \\
& \sqrt{T}\left(\boldsymbol{\mu}\left(\hat{\boldsymbol{\theta}}_{T, m}, \mathbf{0}_{K^{2}(p-m)}, \hat{\boldsymbol{\sigma}}\right)-\boldsymbol{\mu}\left(\boldsymbol{\theta}_{T, m}, \mathbf{0}_{K^{2}(p-m)}, \boldsymbol{\sigma}\right)\right)-\sqrt{T}\left(\boldsymbol{\mu}\left(\boldsymbol{\theta}_{T, p}, \boldsymbol{\sigma}\right)-\boldsymbol{\mu}\left(\boldsymbol{\theta}_{T, m}, \mathbf{0}_{K^{2}(p-m)}, \boldsymbol{\sigma}\right)\right) .
\end{aligned}
$$

The first term in the RHS of Equation (2.36) contains a parameter transformation of the estimated parameters. The first order delta method can be applied to this expression because Theorem 2 explicitly assumes the non-vanishing derivatives at the necessary points. The second term is nonrandom. It is the difference of two terms which only differ in locally misspecified coefficients which have been set to zero. A Taylor expansion can handle this second contribution. The result from the delta method is

$$
\begin{aligned}
& \sqrt{T}\left(\boldsymbol{\mu}\left(\hat{\boldsymbol{\theta}}_{T, m}, \mathbf{0}_{K^{2}(p-m)}, \hat{\boldsymbol{\sigma}}\right)-\boldsymbol{\mu}\left(\boldsymbol{\theta}_{T, m}, \mathbf{0}_{K^{2}(p-m)}, \boldsymbol{\sigma}\right)\right) \\
& \quad \stackrel{d}{\longrightarrow}\left(\frac{\partial \boldsymbol{\mu}\left(\boldsymbol{\theta}_{\infty}, \boldsymbol{\sigma}\right)}{\partial \boldsymbol{\theta}^{\prime}}\left(\boldsymbol{S}_{m} \otimes \boldsymbol{I}_{K}\right)\right)\left(\boldsymbol{A}_{m} \boldsymbol{\delta}+\left(\left[\boldsymbol{S}_{m}^{\prime} \boldsymbol{\Omega} \boldsymbol{S}_{m}\right]^{-1} \boldsymbol{S}_{m}^{\prime} \otimes \boldsymbol{I}_{K}\right) \boldsymbol{R}\right)+\left(\frac{\partial \boldsymbol{\mu}\left(\boldsymbol{\theta}_{\infty}, \boldsymbol{\sigma}\right)}{\partial \boldsymbol{\sigma}^{\prime}}\right) \boldsymbol{S}
\end{aligned}
$$


2 A Focused Information Criterion for Locally Misspecified Vector Autoregressive MODELS

where $\boldsymbol{\theta}_{\infty}$ denotes the parameter vector $\boldsymbol{\theta}_{T, p}$ but with all the misspecification parameters $\boldsymbol{C}_{T}$ set to zero. The result of the Taylor expansion is

$$
\begin{aligned}
& \sqrt{T}\left(\boldsymbol{\mu}\left(\boldsymbol{\theta}_{T, p}, \boldsymbol{\sigma}\right)-\boldsymbol{\mu}\left(\boldsymbol{\theta}_{T, m}, \mathbf{0}_{K^{2}(p-m)}, \boldsymbol{\sigma}\right)\right) \\
& =\sqrt{T} \boldsymbol{\mu}\left(\boldsymbol{\theta}_{T, m}, \boldsymbol{0}_{K^{2}(p-m)}, \boldsymbol{\sigma}\right)+\left(\frac{\partial \boldsymbol{\mu}\left(\boldsymbol{\theta}_{\infty}, \boldsymbol{\sigma}\right)}{\partial \boldsymbol{\theta}^{\prime}}\left(\boldsymbol{S}_{0} \otimes \boldsymbol{I}_{K}\right)\right)\left(\left(\boldsymbol{I}_{K p_{2}}-\boldsymbol{\Pi}_{m}^{\prime} \boldsymbol{\Pi}_{m}\right) \otimes \boldsymbol{I}_{K}\right) \boldsymbol{\delta}+O\left(T^{-1 / 2}\right) \\
& \quad-\sqrt{T} \boldsymbol{\mu}\left(\boldsymbol{\theta}_{T, m}, \mathbf{0}_{K^{2}(p-m)}, \boldsymbol{\sigma}\right) \\
& =\frac{\partial \boldsymbol{\mu}\left(\boldsymbol{\theta}_{\infty}, \boldsymbol{\sigma}\right)}{\partial \boldsymbol{\theta}^{\prime}}\left(\boldsymbol{S}_{0}\left(\boldsymbol{I}_{K p_{2}}-\boldsymbol{\Pi}_{m}^{\prime} \boldsymbol{\Pi}_{m}\right) \otimes \boldsymbol{I}_{K}\right) \boldsymbol{\delta}+O\left(T^{-1 / 2}\right) .
\end{aligned}
$$

The notation can be made a little lighter using the definitions in Theorem 2: $\boldsymbol{D}_{\theta}=$ $\partial \boldsymbol{\mu}\left(\boldsymbol{\theta}_{\infty}, \boldsymbol{\sigma}\right) / \partial \boldsymbol{\theta}^{\prime}$ and $\boldsymbol{D}_{\sigma}=\partial \boldsymbol{\mu}\left(\boldsymbol{\theta}_{\infty}, \boldsymbol{\sigma}\right) / \partial \boldsymbol{\sigma}^{\prime}$. Equations (2.36), (2.37) and (2.38) combine to

$$
\begin{gathered}
\sqrt{T}\left(\boldsymbol{\mu}\left(\hat{\boldsymbol{\theta}}_{T, m}, \mathbf{0}_{p-m}, \hat{\boldsymbol{\sigma}}\right)-\boldsymbol{\mu}\left(\boldsymbol{\theta}_{T, p}, \boldsymbol{\sigma}\right)\right) \\
\stackrel{d}{\longrightarrow} \boldsymbol{D}_{\theta}\left[\left(\boldsymbol{S}_{m} \otimes \boldsymbol{I}_{K}\right) \boldsymbol{A}_{m}-\boldsymbol{S}_{0}\left(\boldsymbol{I}_{K p_{2}}-\boldsymbol{\Pi}_{m}^{\prime} \boldsymbol{\Pi}_{m}\right) \otimes \boldsymbol{I}_{K}\right] \boldsymbol{\delta} \\
\quad+\boldsymbol{D}_{\theta}\left(\boldsymbol{S}_{m}\left[\boldsymbol{S}_{m}^{\prime} \boldsymbol{\Omega} \boldsymbol{S}_{m}\right]^{-1} \boldsymbol{S}_{m}^{\prime} \otimes \boldsymbol{I}_{K}\right) \boldsymbol{R}+\boldsymbol{D}_{\sigma} \boldsymbol{S} \\
=\boldsymbol{D}_{\theta}\left[\left(\boldsymbol{S}_{m}\left[\boldsymbol{S}_{m}^{\prime} \boldsymbol{\Omega} \boldsymbol{S}_{m}\right]^{-1} \boldsymbol{S}_{m}^{\prime} \boldsymbol{\Omega}-\boldsymbol{I}_{K p}\right) \boldsymbol{S}_{0}\left(\boldsymbol{I}_{K p_{2}}-\boldsymbol{\Pi}_{m}^{\prime} \boldsymbol{\Pi}_{m}\right) \otimes \boldsymbol{I}_{K}\right] \boldsymbol{\delta} \\
+\boldsymbol{D}_{\theta}\left(\boldsymbol{S}_{m}\left[\boldsymbol{S}_{m}^{\prime} \boldsymbol{\Omega} \boldsymbol{S}_{m}\right]^{-1} \boldsymbol{S}_{m}^{\prime} \otimes \boldsymbol{I}_{K}\right) \boldsymbol{R}+\boldsymbol{D}_{\sigma} \boldsymbol{S},
\end{gathered}
$$

where the final line follows from the definition of $\boldsymbol{A}_{m}$. With the given definitions of $\boldsymbol{C}_{m}$ and $\boldsymbol{P}_{m}$ we indeed recover the result stated in Theorem 2,

$$
\begin{gathered}
\sqrt{T}\left(\boldsymbol{\mu}\left(\hat{\boldsymbol{\theta}}_{T, m}, \boldsymbol{0}_{K^{2}(p-m)}, \hat{\boldsymbol{\sigma}}\right)-\boldsymbol{\mu}\left(\boldsymbol{\theta}_{T, p}, \boldsymbol{\sigma}\right)\right) \stackrel{d}{\longrightarrow} \boldsymbol{D}_{\theta} \boldsymbol{C}_{m} \boldsymbol{\delta}+\boldsymbol{D}_{\theta} \boldsymbol{P}_{m} \boldsymbol{R}+\boldsymbol{D}_{\sigma} \boldsymbol{S} \\
\sim \mathrm{N}\left(\boldsymbol{D}_{\theta} \boldsymbol{C}_{m} \boldsymbol{\delta}, \boldsymbol{D}_{\theta} \boldsymbol{P}_{m}(\Omega \otimes \boldsymbol{\Sigma}) \boldsymbol{P}_{m} \boldsymbol{D}_{\theta}^{\prime}+\boldsymbol{D}_{\sigma} \boldsymbol{\Xi}_{22} \boldsymbol{D}_{\sigma}^{\prime}\right)
\end{gathered}
$$

Proof of Theorem 3 By Equation (2.40),

$$
\begin{aligned}
\sqrt{T}\left(\bar{\mu}(\boldsymbol{w})-\mu\left(\boldsymbol{\theta}_{T, p}, \boldsymbol{\sigma}\right)\right) & =\sum_{m=p_{1}}^{p} w_{m}\left[\sqrt{T}\left(\mu\left(\hat{\boldsymbol{\theta}}_{T, m}, \mathbf{0}_{K^{2}(p-m)}, \hat{\boldsymbol{\sigma}}\right)-\mu\left(\boldsymbol{\theta}_{T, p}, \boldsymbol{\sigma}\right)\right)\right] \\
& \stackrel{d}{\longrightarrow} \boldsymbol{D}_{\theta} \sum_{m=p_{1}}^{p} w_{m} \boldsymbol{C}_{m} \boldsymbol{\delta}+\boldsymbol{D}_{\theta} \sum_{m=p_{1}}^{p} w_{m} \boldsymbol{P}_{m} \boldsymbol{R}+\boldsymbol{D}_{\sigma} \boldsymbol{S} .
\end{aligned}
$$


The calculation of the mean vector and asymptotic covariance matrix is straightforward.

Proof of Theorem 4 A valid confidence interval for a scalar focus was derived in Theorem 6 of Liu (2015). We follow the same reasoning. Similar to Equation (2.41), we have

$$
\sqrt{T}\left(\overline{\boldsymbol{\mu}}(\hat{\boldsymbol{w}})-\boldsymbol{\mu}\left(\boldsymbol{\theta}_{T}, \boldsymbol{\sigma}\right)\right) \stackrel{d}{\longrightarrow} \boldsymbol{D}_{\theta} \sum_{m=p_{1}}^{p} w_{m}\left(\boldsymbol{R}_{\delta}\right) \boldsymbol{C}_{m} \boldsymbol{R}_{\delta}+\boldsymbol{D}_{\theta}\left(\boldsymbol{\Omega}^{-1} \otimes \boldsymbol{I}_{K}\right) \boldsymbol{R}+\boldsymbol{D}_{\sigma} \boldsymbol{S}
$$

Next, by the convergence of $\hat{\boldsymbol{\delta}}$ to $\boldsymbol{R}_{\delta}$,

$$
\sqrt{T}\left(\overline{\boldsymbol{\mu}}(\hat{\boldsymbol{w}})-\boldsymbol{\mu}\left(\boldsymbol{\theta}_{T}, \boldsymbol{\sigma}\right)\right)-\boldsymbol{D}_{\theta} \sum_{m=p_{1}}^{p} \hat{w}_{m} \hat{\boldsymbol{C}}_{m} \hat{\boldsymbol{\delta}} \stackrel{d}{\longrightarrow} \mathrm{N}\left(\mathbf{0}, \boldsymbol{D}_{\theta}\left(\boldsymbol{\Omega}^{-1} \otimes \boldsymbol{\Sigma}\right) \boldsymbol{D}_{\theta}^{\prime}+\boldsymbol{D}_{\sigma} \boldsymbol{\Xi}_{22} \boldsymbol{D}_{\sigma}^{\prime}\right)
$$

The confidence region is constructed from the standardized quadratic form with population quantities replaced by their consistent estimates.

Proof of Theorem 5 Consider $m \neq p$. We define $\boldsymbol{\alpha}_{m}=\boldsymbol{C}_{m}^{\prime} \boldsymbol{D}_{\theta}^{\prime}, \mathcal{A}=\boldsymbol{S}_{0}^{\prime} \boldsymbol{\Omega}^{-1} \boldsymbol{S}_{0} \otimes \boldsymbol{\Sigma}$, and introduce a standard normally distributed random vector $Z_{K^{2} p_{2}} \sim \mathrm{N}\left(\mathbf{0}, \boldsymbol{I}_{K^{2} p_{2}}\right)$ and random variable $Z \sim \mathrm{N}(0,1)$. Now $\boldsymbol{R}_{\delta}=\boldsymbol{\delta}+\mathcal{A}^{1 / 2} \boldsymbol{Z}_{K^{2} p_{2}}$, and

$$
\begin{aligned}
\boldsymbol{R}_{\delta}^{\prime} \boldsymbol{C}_{m}^{\prime} \boldsymbol{D}_{\theta}^{\prime} \boldsymbol{D}_{\theta} \boldsymbol{C}_{m} \boldsymbol{R}_{\delta} & =\left(\boldsymbol{\alpha}_{m}^{\prime}\left(\boldsymbol{\delta}+\mathcal{A}^{1 / 2} \boldsymbol{Z}_{K^{2} p_{2}}\right)\right)^{2}=\left\|\boldsymbol{\alpha}_{m}^{\prime} \mathcal{A}^{1 / 2}\right\|^{2}\left(\frac{\boldsymbol{\alpha}_{m}^{\prime} \mathcal{A}^{1 / 2} \boldsymbol{Z}_{K^{2} p_{2}}}{\left\|\boldsymbol{\alpha}_{m}^{\prime} \mathcal{A}^{1 / 2}\right\|}+\frac{\boldsymbol{\alpha}_{m}^{\prime} \boldsymbol{\delta}}{\left\|\boldsymbol{\alpha}_{m}^{\prime} \mathcal{A}^{1 / 2}\right\|}\right)^{2} \\
& =\left(\boldsymbol{\alpha}_{m}^{\prime} \mathcal{A} \boldsymbol{\alpha}_{m}\right)\left(Z+\boldsymbol{\alpha}_{m}^{\prime} \boldsymbol{\delta} / \sqrt{\boldsymbol{\alpha}_{m}^{\prime} \mathcal{A} \boldsymbol{\alpha}_{m}}\right)^{2}
\end{aligned}
$$

where $\boldsymbol{\alpha}_{m}^{\prime} \mathcal{A} \boldsymbol{\alpha}_{m}=a_{m}$. The squared expression has the stated noncentral chi-squared distribution (see Chapter 29 of Johnson et al. (1994) for details and moments). Finally, all quantities in $\widehat{F I C}_{p}=D_{\theta}\left(\hat{\Omega}^{-1} \otimes \hat{\Sigma}\right) D_{\theta}^{\prime}+D_{\sigma} \Xi_{22} D_{\sigma}^{\prime}$ are estimated consistently.

Proof of Theorem 6 If either $m=p$ and/or $l=p$, then there is no bias contribution and the matrix elements converge in probability. Now consider $m, l \neq p$, then

(a) For $m=l$, the proof is identical to the proof of Theorem 5, hence omitted.

(b) Start by noting that $\boldsymbol{x}^{\prime} \boldsymbol{A} \boldsymbol{x}=\boldsymbol{x}^{\prime}\left(\frac{\boldsymbol{A}+\boldsymbol{A}^{\prime}}{2}\right) \boldsymbol{x}$, this gives

$$
\boldsymbol{R}_{\delta}^{\prime} \boldsymbol{\alpha}_{m} \boldsymbol{\alpha}_{l}^{\prime} \boldsymbol{R}_{\delta}=\left(\mathcal{A}^{-1 / 2} \boldsymbol{\delta}+Z_{K^{2} p_{2}}\right)^{\prime}\left[\mathcal{A}^{1 / 2}\left(\frac{\boldsymbol{\alpha}_{m} \boldsymbol{\alpha}_{l}^{\prime}+\boldsymbol{\alpha}_{l} \boldsymbol{\alpha}_{m}^{\prime}}{2}\right) \mathcal{A}^{1 / 2}\right]\left(\mathcal{A}^{-1 / 2} \boldsymbol{\delta}+Z_{K^{2} p_{2}}\right)
$$


We subsequently use the transformation stated in Imhof (1961). The matrix in square brackets is symmetric and has a rank of at most two. The eigenvalue decomposition mentioned in Theorem 6 applies, and therefore

$$
\boldsymbol{R}_{\delta}^{\prime} \boldsymbol{\alpha}_{m} \boldsymbol{\alpha}_{l}^{\prime} \boldsymbol{R}_{\delta}=\sum_{i=1}^{2} \lambda_{i}\left(\boldsymbol{v}_{i}^{\prime} \mathcal{A}^{-1 / 2} \boldsymbol{\delta}+Z_{i}\right)^{2} \sim \sum_{i=1}^{2} \lambda_{i} \chi_{\text {noncentral }}^{2}\left(1,\left(\boldsymbol{v}_{i}^{\prime} \mathcal{A}^{-1 / 2} \boldsymbol{\delta}\right)^{2}\right)
$$

where the independence of the $Z_{i}$ follows from orthonormality of the eigenvectors.

\section{B Derivation of the Optimal Weights in the Simplified Model}

The simplified model is $y_{t}=\alpha y_{t-1}+\frac{\delta}{\sqrt{T}} y_{t-2}+u_{t}$. We develop the elements of the matrix $\boldsymbol{\Psi}^{\infty}$ for the case with bias correction.

$$
\begin{aligned}
\boldsymbol{\Psi}_{1,1}^{\infty} & =a_{1} \chi_{\text {noncentral }}^{2}\left(1,\left(\boldsymbol{D}_{\theta} \boldsymbol{C}_{1} \boldsymbol{\delta}\right)^{2} / a_{1}\right)-a_{1}+\sigma^{2} \boldsymbol{D}_{\theta} \boldsymbol{P}_{1} \boldsymbol{\Omega} \boldsymbol{P}_{1} \boldsymbol{D}_{\theta}^{\prime} \\
& =a_{1} \chi_{\text {noncentral }}^{2}\left(1,\left(\boldsymbol{D}_{\theta} \boldsymbol{C}_{1} \boldsymbol{\delta}\right)^{2} / a_{1}\right)-a_{1}+\sigma^{2} \boldsymbol{D}_{\theta} \boldsymbol{S}_{1}\left[\boldsymbol{S}_{1}^{\prime} \boldsymbol{\Omega} \boldsymbol{S}_{1}\right]^{-1} \boldsymbol{S}_{1}^{\prime} \boldsymbol{D}_{\theta}^{\prime} \\
& =a_{1} \chi_{\text {noncentral }}^{2}\left(1,\left(\boldsymbol{D}_{\theta} \boldsymbol{C}_{1} \boldsymbol{\delta}\right)^{2} / a_{1}\right)-a_{1}+\sigma^{2} \boldsymbol{D}_{\theta} \boldsymbol{P}_{1} \boldsymbol{D}_{\theta}^{\prime} \\
\boldsymbol{\Psi}_{1,2}^{\infty} & =\boldsymbol{D}_{\theta} \boldsymbol{P}_{1}\left(\sigma^{2} \boldsymbol{\Omega}\right) \boldsymbol{P}_{2} \boldsymbol{D}_{\theta}^{\prime}=\sigma^{2} \boldsymbol{D}_{\theta} \boldsymbol{P}_{1} \boldsymbol{D}_{\theta}^{\prime} \\
\boldsymbol{\Psi}_{2,2}^{\infty} & =\boldsymbol{D}_{\theta} \boldsymbol{P}_{2}\left(\sigma^{2} \boldsymbol{\Omega}\right) \boldsymbol{P}_{2} \boldsymbol{D}_{\theta}^{\prime}=\sigma^{2} \boldsymbol{D}_{\theta} \boldsymbol{\Omega}^{-1} \boldsymbol{D}_{\theta}^{\prime}
\end{aligned}
$$

The solution to the optimization problem will not change if we subtract a constant from every element. We subtract $\sigma^{2} \boldsymbol{D}_{\theta} \boldsymbol{P}_{1} \boldsymbol{D}_{\theta}^{\prime}$, and denote the result by $\tilde{\boldsymbol{\Psi}}^{\infty}$. The resulting matrix looks like

$$
\tilde{\boldsymbol{\Psi}}^{\infty}=\left[\begin{array}{cc}
a_{1} \chi_{\text {noncentral }}^{2}\left(1,\left(\boldsymbol{D}_{\theta} \boldsymbol{C}_{1} \boldsymbol{\delta}\right)^{2} / a_{1}\right)-a_{1} & 0 \\
0 & \sigma^{2} \boldsymbol{D}_{\theta}\left(\boldsymbol{\Omega}^{-1}-\boldsymbol{P}_{1}\right) \boldsymbol{D}_{\theta}^{\prime}
\end{array}\right] .
$$

The $(1,1)$-element of this matrix is negative whenever $\chi_{\text {noncentral }}^{2}\left(1,\left(\boldsymbol{D}_{\theta} \boldsymbol{C}_{1} \boldsymbol{\delta}\right)^{2} / a_{1}\right)<1$. From this point onwards, we consider the case without bias correction. Now,

$$
\tilde{\boldsymbol{\Psi}}^{\infty}=\left[\begin{array}{cc}
a_{1} \chi_{\text {noncentral }}^{2}\left(1,\left(\boldsymbol{D}_{\theta} \boldsymbol{C}_{1} \boldsymbol{\delta}\right)^{2} / a_{1}\right) & 0 \\
0 & \sigma^{2} \boldsymbol{D}_{\theta}\left(\boldsymbol{\Omega}^{-1}-\boldsymbol{P}_{1}\right) \boldsymbol{D}_{\theta}^{\prime}
\end{array}\right]:=\left[\begin{array}{cc}
\kappa_{1} & 0 \\
0 & \kappa_{2}
\end{array}\right],
$$


and if we assume $\boldsymbol{D}_{\theta}\left(\boldsymbol{\Omega}^{-1}-\boldsymbol{P}_{1}\right) \boldsymbol{D}_{\theta}^{\prime}>0$ (see footnote 13 ), then we have $\tilde{\boldsymbol{\Psi}}^{\infty}>0$ and hence unique weights. The optimal weight $w^{*}$ is the minimizer of $\kappa_{1} w^{2}+\kappa_{2}(1-w)^{2}$ subject to $0 \leq w \leq 1$. The unconstrained solution is

$$
w^{*}=\frac{\kappa_{2}}{\kappa_{1}+\kappa_{2}}=\frac{\sigma^{2} \boldsymbol{D}_{\theta}\left(\boldsymbol{\Omega}^{-1}-\boldsymbol{P}_{1}\right) \boldsymbol{D}_{\theta}^{\prime}}{a_{1} \chi_{\text {noncentral }}^{2}\left(1,\left(\boldsymbol{D}_{\theta} \boldsymbol{C}_{1} \boldsymbol{\delta}\right)^{2} / a_{1}\right)+\sigma^{2} \boldsymbol{D}_{\theta}\left(\boldsymbol{\Omega}^{-1}-\boldsymbol{P}_{1}\right) \boldsymbol{D}_{\theta}^{\prime}} .
$$

This solution is both positive and in the interval $[0,1]$ because $\kappa_{1}, \kappa_{2}>0$. The constraint $w \in[0,1]$ is thus automatically satisfied. It follows that

$$
\begin{aligned}
\operatorname{Pr}\left(w^{*} \leq x\right) & =\operatorname{Pr}\left(\frac{\kappa_{2}}{\kappa_{1}+\kappa_{2}} \leq x\right)=\operatorname{Pr}\left(\kappa_{1} \geq \frac{\kappa_{2}[1-x]}{x}\right) \\
& =\operatorname{Pr}\left(\chi_{\text {noncentral }}^{2}\left(1,\left(\boldsymbol{D}_{\theta} \boldsymbol{C}_{1} \boldsymbol{\delta}\right)^{2} / a_{1}\right) \geq \frac{\sigma^{2} \boldsymbol{D}_{\theta}\left(\boldsymbol{\Omega}^{-1}-\boldsymbol{P}_{1}\right) \boldsymbol{D}_{\theta}^{\prime}[1-x]}{a_{1} x}\right) .
\end{aligned}
$$

\section{C No Gradient Dependence in Simplified Model}

For $y_{t}=\alpha y_{t-1}+\frac{\delta}{\sqrt{T}} y_{t-2}+u_{t}$, we have $\Omega=\frac{\sigma^{2}}{1-\alpha^{2}}\left[\begin{array}{cc}1 & \alpha \\ \alpha & 1\end{array}\right]$ and $\Omega^{-1}=\frac{1}{\sigma^{2}}\left[\begin{array}{cc}1 & -\alpha \\ -\alpha & 1\end{array}\right]$. The required selection matrices are $\boldsymbol{S}_{1}=\left[\begin{array}{l}1 \\ 0\end{array}\right], \boldsymbol{S}_{0}=\left[\begin{array}{l}0 \\ 1\end{array}\right]$, and $\boldsymbol{\Pi}_{1}^{\prime} \boldsymbol{\Pi}_{1}=\mathbf{O}_{2 \times 2}$. Straightforward calculations show

$$
\boldsymbol{C}_{1}=\left\{\left[\begin{array}{ll}
1 & \alpha \\
0 & 0
\end{array}\right]-\boldsymbol{I}_{2}\right\}\left[\begin{array}{l}
0 \\
1
\end{array}\right]=\left[\begin{array}{c}
\alpha \\
-1
\end{array}\right]:=\boldsymbol{y}
$$

and

$$
\boldsymbol{\Omega}^{-1}-\boldsymbol{P}_{1}=\frac{1}{\sigma^{2}}\left[\begin{array}{cc}
\alpha^{2} & -\alpha \\
-\alpha & 1
\end{array}\right]=\frac{1}{\sigma^{2}}\left[\begin{array}{c}
\alpha \\
-1
\end{array}\right]\left[\begin{array}{ll}
\alpha & -1
\end{array}\right]=\frac{1}{\sigma^{2}} \boldsymbol{y} \boldsymbol{y}^{\prime}
$$

The elements of the weighting matrix (with bias correction in red) are now given by:

$$
\begin{aligned}
& \boldsymbol{\Psi}_{11}=\boldsymbol{D}_{\theta}\left[\boldsymbol{C}_{1}\left(\boldsymbol{\delta} \boldsymbol{\delta}-\sigma^{2} \boldsymbol{S}_{0}^{\prime} \Omega^{-1} \boldsymbol{S}_{0}\right)^{\prime} \boldsymbol{C}_{1}^{\prime}+\sigma^{2} \boldsymbol{P}_{1}\right] \boldsymbol{D}_{\theta}^{\prime}=\left(\delta^{2}-1\right)\left(\boldsymbol{D}_{\theta} \boldsymbol{y}\right)^{2}+\sigma^{2} \boldsymbol{D}_{\theta} \boldsymbol{P}_{1} \boldsymbol{D}_{\theta}^{\prime}, \\
& \boldsymbol{\Psi}_{12}=\sigma^{2} \boldsymbol{D}_{\theta} \boldsymbol{P}_{1} \boldsymbol{D}_{\theta}^{\prime}, \\
& \boldsymbol{\Psi}_{22}=\sigma^{2} \boldsymbol{D}_{\theta}\left(\boldsymbol{\Omega}^{-1}-\boldsymbol{P}_{1}\right) \boldsymbol{D}_{\theta}^{\prime}+\sigma^{2} \boldsymbol{D}_{\theta} \boldsymbol{P}_{1} \boldsymbol{D}_{\theta}^{\prime}=\left(\boldsymbol{D}_{\theta} \boldsymbol{y}\right)^{2}+\sigma^{2} \boldsymbol{D}_{\theta} \boldsymbol{P}_{1} \boldsymbol{D}_{\theta}^{\prime} .
\end{aligned}
$$


2 A Focused Information Criterion for Locally Misspecified Vector Autoregressive MODELS

The weights are determined from

$$
\begin{aligned}
\boldsymbol{w}^{0} & =\arg \min _{\boldsymbol{w} \in \mathcal{H}} \boldsymbol{w}^{\prime} \boldsymbol{\Psi} \boldsymbol{w}=\arg \min _{\boldsymbol{w} \in \mathcal{H}}\left(\boldsymbol{D}_{\theta} \boldsymbol{y}\right)^{2} \boldsymbol{w}^{\prime}\left[\begin{array}{cc}
\left(\delta^{2}-1\right) & 0 \\
0 & 1
\end{array}\right] \boldsymbol{w}+\sigma^{2} \boldsymbol{D}_{\theta} \boldsymbol{P}_{1} \boldsymbol{D}_{\theta}^{\prime} \\
& =\arg \min _{0 \leq w \leq 1}\left(\boldsymbol{D}_{\theta} \boldsymbol{y}\right)^{2}\left[w^{2}\left(\delta^{2}-1\right)+(1-w)^{2}\right]+\sigma^{2} \boldsymbol{D}_{\theta} \boldsymbol{P}_{1} \boldsymbol{D}_{\theta}^{\prime}
\end{aligned}
$$

This expression shows that the weights do not depend on the quantity of interest because $\boldsymbol{D}_{\theta}$ is no longer of importance for the optimal weight calculation.

Remark 2.11 The diagonal elements of the weighting matrix without bias correction are also the AMSE's of the individual model. We would prefer the model with one lag if $\delta^{2}<1$. This is supported by Figure 2.1. 


\section{D Simulations for Multiple Focus Parameters}

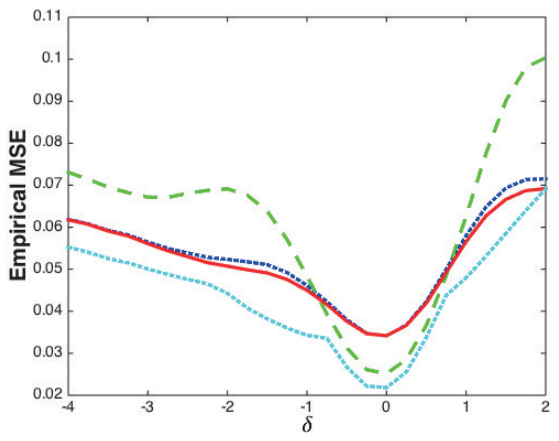

(a) Model Selection, $T=100$.

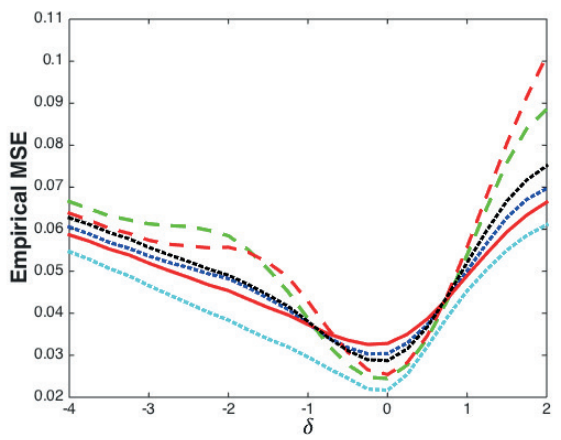

(c) Model Averaging, $T=100$.

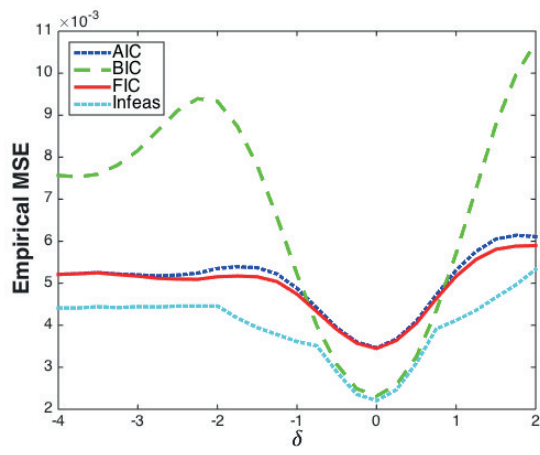

(b) Model Selection, $T=1000$.

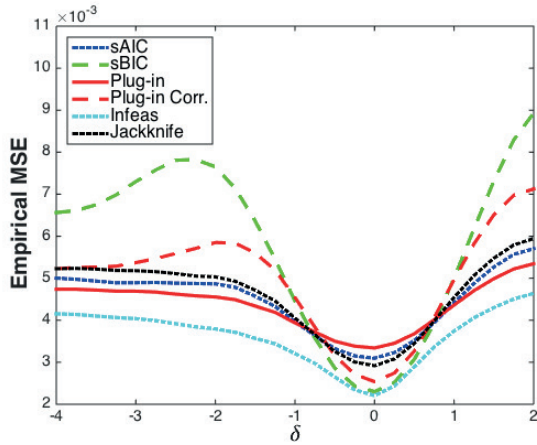

(d) Model Averaging, $T=1000$.

Figure 2.11: Simulation results for a multiple quantities of interest. Model selection/averaging is based on the impulse responses from horizon 1 up to 6. The trace of the AMSE matrix is used to map the AMSE matrix to a scalar, i.e. model selection/averaging is based on the sum of the asymptotic mean-squared errors. Therefore, we also report the sum of the empirical MSE of the first six horizons on the vertical axis. 



\section{Chapter 3}

\section{Estimating VAR models by Least Absolute Deviations}

"If we have been deceived by the appearance of discordance, and the facility-curve was really a normal probability-curve, yet we shall have lost little by taking the median instead of the arithmetic mean. For the error of the former is of the same order as (only

1.3 greater than) the error of the latter. And, if the observations are really discordant, the derangement due to the larger deviations will not be serious, as it is for the arithmetic mean."

- Francis Edgeworth (1845-1926) 


\section{Abstract}

This chapter considers the estimation of stable vector autoregressive (VAR) models by least absolute deviations (LAD). The proposed estimator can be obtained as the solution of a linear program. Portnoy and Koenker (1997) have pointed out that computational costs are no longer valid arguments in favor of an ordinary least squares (OLS) approach. It is also known since Edgeworth (1887) that least squares estimation looses its appeal in the presence of fat-tailed innovation distributions and/or the presence of outliers. The first part of this paper provides the necessary conditions and proves the consistency and asymptotic normality of the LAD estimator under (possible) linear restrictions. The second part extends earlier work by Koenker and Bassett (1982) and Weiss (1991) by examining LAD versions of the Wald, Lagrange Multiplier and Likelihood Ratio test in the VAR setup. These test are derived under a likelihood function that assumes i.i.d. Laplacian innovations but more primitive conditions are stated that allow for a pseudo-likelihood interpretation. The relevance of such tests should be clear in view of the importance of testing for Granger causality and lag order determination. Thirdly and finally, we look at LAD-based information criteria to determine the lag order of VAR models. Monte Carlo simulations demonstrate reductions in mean-squared errors and gains in test power for leptokurtic or contaminated innovation distributions. The sizes of the tests are close to the desired nominal level. The simulations also reveal that the introduced information criterion with BIC-penalty for model complexity selects the true lag order with high frequency. 


\subsection{Introduction}

The vector autoregressions introduced by Sims (1980) offer a popular and coherent approach to capture the dynamics in multiple time series. A description of the strengths and weaknesses of VAR's can be found in Stock and Watson (2001). Traditionally, as motivated by computational considerations, inference is based on a quadratic loss function. That is, parameter estimation, hypothesis tests, impulse response analysis, and the determination of the lag order are all four based on the least squares estimates.

This paper develops statistical methods for VAR models based on least absolute deviations (LAD). It has been realized that LAD estimators (hence also our estimator) are solutions of linear programs. An overview of solution approaches can be found in Bloomfield and Steiger (1983). Portnoy and Koenker (1997) show that LAD estimation is computationally as fast as OLS estimation, or even faster. Our LAD estimates are shown to be asymptotically normally distributed with a limiting covariance matrix that can be consistently estimated. This paves the way for hypothesis testing. We show that the Lagrange Multiplier and Wald test statistics are asymptotically pivotal, whereas the Likelihood Ratio test statistic is not. These first two test statistics will thus allow us to test linear hypotheses. We also consider information criteria based on LAD, and show that these provide consistent lag order selection. We now proceed with a discussion of several results in the literature that also take an LAD approach to time series estimation. A distinction is made between single-equation and multiple time series models.

Two early references on LAD estimation are Powell (1984) and Weiss (1991). Powell (1984) considers LAD estimation in a censored i.i.d. regression framework. Weiss (1991) extends the asymptotic analysis of Powell (1984) and derives both asymptotics and hypothesis tests for nonlinear dynamic models under mixing conditions. Our results are also obtained under mixingale conditions. We also mention four other publications that entail single-equation models on quantile estimation: Engle and Manganelli (2004), Xiao (2009), Koenker and Xiao (2004), and Cho et al. (2015). We stress that LAD and quantile estimation at the median are computationally the same. However, there is one important difference between the two. The underlying process has to be stationary and ergodic to consistently estimate (conditional) quantiles, whereas mixingales (with conditional median zero) are still allowed for consistent LAD estimation. The paper by Engle and Manganelli (2004) deals with conditional autoregressive value at risk models (abbreviated as CAViaR). Xiao (2009) and Cho et al. (2015) discuss quantile estimation in a cointegration setting. The setting by Xiao (2009) is a single equation cointegration 
framework with stationary errors, whereas Cho et al. (2015) allow lags of the dependent variable to enter the cointegrating model as well. Koenker and Xiao (2004) are concerned with unit root tests based on quantile estimation.

The time series ${ }^{1}$ literature on multivariate least absolute deviations or quantile estimation is rather scarce. Particularly related to our work is the paper by White et al. (2015). This paper can be considered a multivariate extension of Engle and Manganelli (2004). That is, they consider estimation of multi-equation conditional quantile models. As an additional feature they also introduce quantile dependent impulse responses.

\subsection{The Estimator}

To fix the notation we will consider the stable $K$-dimensional $\operatorname{VAR}(p)$ process

$$
\boldsymbol{y}_{t}=\boldsymbol{\nu}+\boldsymbol{A}_{1} \boldsymbol{y}_{t-1}+\cdots+\boldsymbol{A}_{p} \boldsymbol{y}_{t-p}+\boldsymbol{u}_{t}
$$

with the observed series $\boldsymbol{y}_{t}=\left(y_{1 t}, \ldots, y_{K t}\right)^{\prime}$, the $\boldsymbol{A}_{i}$ denoting $(K \times K)$ coefficient matrices and $\boldsymbol{u}_{t}=\left(u_{1}, \ldots, u_{K t}\right)^{\prime}$ denoting the innovations. We will be concerned with stable vector autoregressions. Some definitions are required before we can make the previous statement mathematically precise. In line with the notation in Lütkepohl (2005), we define

$$
\begin{aligned}
\boldsymbol{Y}:=\left(\boldsymbol{y}_{1}, \ldots, \boldsymbol{y}_{T}\right) & (K \times T), \\
\boldsymbol{B}:=\left(\boldsymbol{\nu}, \boldsymbol{A}_{1}, \ldots, \boldsymbol{A}_{p}\right) & (K \times(K p+1)), \\
\boldsymbol{z}_{t}:=\left(1, \boldsymbol{y}_{t}^{\prime}, \ldots, \boldsymbol{y}_{t-p+1}^{\prime}\right)^{\prime} & ((K p+1) \times 1), \\
\boldsymbol{Z}:=\left(\boldsymbol{z}_{0}, \ldots, \boldsymbol{z}_{T-1}\right) & ((K p+1) \times T), \\
\boldsymbol{U}:=\left(\boldsymbol{u}_{1}, \ldots, \boldsymbol{u}_{T}\right) & (K \times T),
\end{aligned}
$$

and write Equation (3.1) as $\boldsymbol{y}_{t}=\boldsymbol{B} \boldsymbol{z}_{t-1}+\boldsymbol{u}_{t}$. We stack all the parameters equation-wise in the vector $\boldsymbol{b}=\operatorname{vec}\left(\boldsymbol{B}^{\prime}\right)$. This parameter vector $\boldsymbol{b}$ can be subjected to linear constraints. We therefore write $\boldsymbol{b}=\boldsymbol{R} \gamma+\boldsymbol{r}$ for some known $(K(K p+1) \times M)$ matrix $\boldsymbol{R}$ and a known vector $\boldsymbol{r}$ of length $K(K p+1)$. Our aim is to conduct inference on the unknown parameter vector $\gamma$. This parameter vector $\gamma$ is an element in the parameter space $\Gamma$. The following assumptions are maintained throughout this paper.

\footnotetext{
${ }^{1}$ Additional references on multivariate LAD or quantile estimation in i.i.d. settings are given at the appropriate places in the main text.
} 
Assumption 3.1 (Stability Condition) Consider the set of matrices $\left\{\boldsymbol{A}_{1}, \ldots, \boldsymbol{A}_{p}\right\}$ implied by the definition in Equation (3.2) and $\boldsymbol{b}=\boldsymbol{R} \gamma+\boldsymbol{r}$. For all $\gamma \in \Gamma$ we have $\operatorname{det}(\boldsymbol{A}(z))=$ $\operatorname{det}\left(\boldsymbol{I}_{K} z^{p}-\boldsymbol{A}_{1} z^{p-1}-\ldots-\boldsymbol{A}_{p}\right) \neq 0$ for all $|z| \geq 1$.

Assumption 3.2 (Innovations 1) The process $\left\{\boldsymbol{u}_{t}\right\}$ satisfies the conditions:

(a) The components of $\left\{\boldsymbol{u}_{t}\right\}$ are either uniform mixing with $\phi_{m}$ of size $-r /(2 r-2), r \geq 2$ or strong mixing with $\alpha_{m}$ of size $-r /(r-2)$ with $r>2$.

(b) $\mathrm{E}\left\|\boldsymbol{u}_{t}\right\|^{2+\delta}<\infty$ for some $\delta>0$ and all $t$.

Assumption 3.3 (Identifiable Uniqueness) Parameter identifiability ${ }^{2}$ is guaranteed by:

(a) Let $\mathcal{F}_{t-1}=\sigma\left(\boldsymbol{u}_{-\infty}, \ldots, \boldsymbol{u}_{t-1}\right)$ denote $\sigma$-field containing the information up to time $t-1$. For every $k=1, \ldots, K$ and conditionally on $\mathcal{F}_{t-1}$ the innovations $u_{k t}$ have median zero, and with probability approaching 1 , their densities $f_{k, t}$ are bounded away from zero, i.e.

$$
\inf _{t} \min _{k=1,2, \ldots, K} f_{k, t}(x)>0
$$

for a neighborhood around $x=0$.

(b) $\frac{1}{T} \sum_{t=1}^{T} \mathrm{E}\left(\boldsymbol{z}_{t-1} \boldsymbol{z}_{t-1}^{\prime}\right)$ converges to a positive definite matrix.

Assumption 3.1 makes sure that the vector autoregression is stable (neither unit roots nor explosive behavior). The process $\left\{\boldsymbol{y}_{t}\right\}$ is neither strictly nor weakly stationary because we do not impose i.i.d. innovations. Assumption 3.3(a) only requires the conditional median to be correctly specified. Other distributional properties such as the variances and densities conditional on $\mathcal{F}_{t-1}$ can thus vary over time. The mixing and moment conditions of Assumption 3.2 are necessary to control the serial dependency and heterogeneity such that a law of large numbers can be invoked. Assumption 3.3(b) is a more technical assumption and is used to identify the parameters. This assumption is also encountered in least squares estimation.

\footnotetext{
${ }^{2}$ The parameter identifiability that is used here relates to identifiable uniqueness, e.g. definition 3.2 on page 19 of Gallant and White (1988). It requires that the limit of the objective function is uniquely minimized at the true parameters. This should not be confused with parameter identifiability in the likelihood framework where we say that the parameters are identified if different parameters imply a different likelihood.
} 
Having discussed the asumptions, we now proceed to the estimator. The estimator is defined as $^{3}$

$$
\begin{aligned}
\widehat{\gamma}_{T} & =\arg \min _{\boldsymbol{\gamma} \in \Gamma} \frac{1}{T} \sum_{t=1}^{T}\left\|\boldsymbol{y}_{t}-\left(I_{K} \otimes \boldsymbol{z}_{t-1}^{\prime}\right)(\boldsymbol{R} \boldsymbol{\gamma}+\boldsymbol{r})\right\|_{1} \\
& =\arg \min _{\boldsymbol{\gamma} \in \Gamma} \frac{1}{T} \sum_{t=1}^{T} \sum_{k=1}^{K}\left|y_{k t}-\boldsymbol{z}_{t-1}^{\prime}\left(\boldsymbol{R}_{k} \boldsymbol{\gamma}+\boldsymbol{r}_{k}\right)\right|
\end{aligned}
$$

where $\boldsymbol{R}_{k}$ and $\boldsymbol{r}_{k}$ denote the rows of $\boldsymbol{R}$ and $\boldsymbol{r}$ belonging to equation $k$, respectively. Note how the least squares estimator minimizes the same objective function with the exception of absolute deviations being replaced by squared deviations. In other words, we are using a different metric to measure deviations between the observed values $\boldsymbol{y}_{t}$ and the fitted values $\boldsymbol{z}_{t-1}^{\prime}\left(\boldsymbol{R}_{k} \gamma+\boldsymbol{r}_{k}\right)$. The minimization of squared deviations is optimal in the meansquared sense under Gaussian innovations. The performance of least squares is known to be less satisfactory under departures from normality, e.g. in the presence of fat tailed innovations or outliers. The quote by Edgeworth (1887) at the start of this chapter stresses this point. Alternatively, the estimator can be motivated as a maximum likelihood estimator if one assumes the innovations $\left\{u_{k t}\right\}$ to be Laplace random variables and i.i.d. over both time and cross-section. ${ }^{4}$ This interpretation is stressed in the second line of Equation (3.3).

Remark 3.1 The estimator $\widehat{\gamma}_{T}$ as defined by Equation (3.3) can be obtained as the solution of a linear program in $M+K T$ unknowns. This implies that computations are fast in comparison to other robust methods which require the minimization of other nonlinear objective functions (e.g. Terpstra and Rao (2002), Reber et al. (2008), and Muler and Yohai (2013)). If the parameter constraints do not involve several cross-sections, then estimation can proceed equation-by-equation. Further details on the implementation are given in Appendix 3.A.1.

Remark 3.2 The asymptotic results are derived under linear restrictions on the parameter estimates. Such linear restrictions are common in Granger-causality tests, sequential tests to determine the lag order and/or from the exclusion of insignificant parameters from a previous estimation. The asymptotic results on the constrained estimates can be found in the next section. Tests for the validity of these restrictions are discussed in Section 3.2.2.

\footnotetext{
${ }^{3}$ There is a slight abuse of notation because the minimizer of the objective function might not be unique.

${ }^{4}$ The estimator coincides with the maximum likelihood estimator if Laplacian innovations are assumed. However, this distributional assumption is not needed as long as Assumptions 1-3 are valid. Under different innovation distributions we might view the estimator as a quasi-maximum likelihood estimator.
} 


\subsubsection{Asymptotic Properties of the LAD Estimator}

We now mathematically formalize our setup in order to derive asymptotic properties. Let $(\Omega, \mathscr{F}, \mathbb{P})$ be a complete probability space. For some $\omega \in \Omega$ we have observed $\boldsymbol{y}_{t}$ for $t=1, \ldots, T$. This series of sample size $T$ has been generated by the vector autoregressive model of Equation (3.1) with the parameters satisfying $\boldsymbol{b}_{0}=\boldsymbol{R} \gamma_{0}+\boldsymbol{r}$ with $\boldsymbol{R}$ and $\boldsymbol{r}$ known. We will refer to $\gamma_{0} \in \Gamma$ as the true parameter vector. We require one additional assumption to establish consistency of $\widehat{\gamma}_{T}$.

\section{Assumption 3.4 (Compactness of the Parameter Space) $\Gamma$ is a compact subset of $\mathbb{R}^{M}$.}

The relevance/necessity of Assumption 3.4 is perhaps best appreciated when embedded in a sketch of the consistency proof. The compactness assumption enters the consistency proof on two occasions. Firstly, the compactness of the parameter space and the continuity of the objective function ensure that the minimizer $\widehat{\gamma}_{T}$ is measurable. A discussion is provided in Bierens (1981). Secondly, there is no closed form expression for $\widehat{\gamma}_{T}$ and we therefore rely on results for M-estimators (see for example: chapter 3 of Gallant and White (1988), or chapter 5 of van der Vaart (2000)). Consistency proofs for these estimators typically involve two requirements: uniform convergence of the objective function and identifiable uniqueness of the parameter vector. The latter requirement is covered by Assumption 3.3. It is the uniform convergence result which also exploits the compactness of the parameter space. One typically controls the approximation error for small neighborhoods of an arbitrary point in the parameter space (see Andrews (1987) and Pötscher and Prucha (1989) for examples). The compactness assumption subsequently makes sure that a finite number of these neighborhoods covers the parameter space. A uniform convergence result follows easily since this approximation error occurs only a finite number of times.

It is not obvious how to parameterize $\Gamma$ in practice because it is not straightforward to give a closed form expression for the set of parameter which result in stable vector autoregressions. Such conditions are only known for univariate processes with low order. As an illustration, the $\operatorname{AR}(1)$ process $y_{t}=\alpha y_{t-1}+\epsilon_{t}$ is stable for $|\alpha|<1$. Assumptions 3.1 and 3.4 therefore hold if $\Gamma=[-1+h, 1-h]$ for some $h>0$. We have discussed the assumptions and sketched the proof of Theorem 3.1.

Theorem 3.1 (Consistency) Suppose that Assumptions 1-4 hold. Then, we have $\widehat{\gamma}_{T} \rightarrow \gamma_{0}$ almost surely. 
Additional assumptions are needed to obtain an asymptotic normality result. These assumptions are listed below as Assumption 3.5, 3.6, and 3.7. These assumptions and the asymptotic normality result, Theorem 3.2, will be motivated along the lines of Phillips (1991). We cite Phillips (1991) in stating that, 'Our approach is heuristic and we do not claim to deal rigorously with all of the mathematical issues that arise.' (first line of the final paragraph on page 451). A rigorous proof based on Huber (1967) is provided in Appendix 3.A.3. The momentary loss of rigor is compensated by: (1) the added intuition due to similarities to standard asymptotic normality proofs, and (2) a more natural introduction to the various definitions that accompany the normality result. The similarities and differences with the approach pioneered by Huber (1967) are discussed in Remark 3.3 .

Assumption 3.5 (True Parameter in the Interior of the Parameter Space) $\gamma_{0}$ is interior to $\Gamma$.

Assumption 3.6 (Innovations 2) For $k \in\{1,2, \ldots, K\}$, let $f_{k, t}$ denote the density of $u_{k t}$ conditional on $\mathcal{F}_{t-1}$. We assume with probability approaching 1 , that:

(a) The conditional density $f_{k, t}$ is uniformly bounded, i.e.

$$
f_{0}=\sup _{x, t} \max _{k=1,2, \ldots, K} f_{k, t}(x)<\infty .
$$

(b) $f_{k, t}$ is Lipschitz continuous uniformly in $t$ and in every $k \in\{1,2, \ldots, K\}$. That is, for every $x_{1}$ and $x_{2}$, there is a finite positive constant $L_{0}$ neither depending on $t$ nor $k$, such that $\left|f_{k, t}\left(x_{1}\right)-f_{k, t}\left(x_{2}\right)\right| \leq L_{0}\left|x_{1}-x_{2}\right|$.

Assumption 3.7 (Nondegenerate Asymptotic Covariance Matrix) There exists an integer $T_{0}$, such that the matrices

(a) $\overline{\boldsymbol{V}}_{T}=\frac{1}{T} \sum_{t=1}^{T} \mathrm{E}\left(\boldsymbol{\Psi}_{t} \otimes \boldsymbol{z}_{t-1} \boldsymbol{z}_{t-1}^{\prime}\right)$,

(b) $\overline{\boldsymbol{H}}_{T}=\frac{2}{T} \sum_{t=1}^{T} \mathrm{E}\left(\boldsymbol{\Xi}_{t} \otimes \boldsymbol{z}_{t-1} \boldsymbol{z}_{t-1}^{\prime}\right)$,

are positive definite for all $T \geq T_{0}$ where $\boldsymbol{\Psi}_{t}$ denotes the matrix having $(i, j)$ 'th element $\boldsymbol{\Psi}_{t, i j}=\mathrm{E}\left(\operatorname{sgn}\left(u_{i t}\right) \operatorname{sgn}\left(u_{j t}\right) \mid \mathcal{F}_{t-1}\right)$ and $\boldsymbol{\Xi}_{t}=\operatorname{diag}\left(f_{1, t}(0), f_{2, t}(0), \ldots, f_{K, t}(0)\right)$.

The heuristic proof in Phillips (1991) treats the objective function in Equation (3.3) as a differentiable function in which the derivatives are generalized functions. That is, 
we assume that the following derivatives are valid for any value of $x: \frac{\partial}{\partial x}|x|=\operatorname{sgn}(x)$, and $\frac{\partial^{2}}{\partial x^{2}}|x|=2 \delta(x) .{ }^{5}$ The generalized function $\delta(x)$ is referred to as the Dirac delta function and is defined implicitly by its property $\int_{-\infty}^{\infty} \delta(x) f(x) d x=f(0)$ for any continuous function $f$. We are now in the possession of two 'derivatives' and can therefore fall back on standard asymptotic normality proofs (e.g. Newey and McFadden (1994)). The first order condition reads

$$
-\frac{1}{T} \sum_{t=1}^{T} \sum_{k=1}^{K} \boldsymbol{R}_{k}^{\prime} z_{t-1} \operatorname{sgn}\left(y_{k t}-\boldsymbol{z}_{t-1}^{\prime}\left(\boldsymbol{R}_{k} \widehat{\gamma}_{T}+\boldsymbol{r}_{k}\right)\right)=\mathbf{0}
$$

Assumption 3.5 places the true parameter in the interior of the parameter space. There hence exists a neighborhood of $\gamma_{0}$ which is contained in $\Gamma$. Applying a mean-value theorem to each individual element on the RHS of Equation (3.5), and multiplying by $\sqrt{T}$ gives

$$
\begin{aligned}
\mathbf{0}=\frac{1}{\sqrt{T}} & \sum_{t=1}^{T} \sum_{k=1}^{K} \boldsymbol{R}_{k}^{\prime} \boldsymbol{z}_{t-1} \operatorname{sgn}\left(u_{k t}\right) \\
& +\left(\frac{2}{T} \sum_{t=1}^{T} \sum_{k=1}^{K} \delta\left(y_{k t}-\boldsymbol{z}_{t-1}^{\prime}\left(\boldsymbol{R}_{k} \widetilde{\gamma}_{T}+\boldsymbol{r}_{k}\right)\right) \boldsymbol{R}_{k}^{\prime} \boldsymbol{z}_{t-1} \boldsymbol{z}_{t-1}^{\prime} \boldsymbol{R}_{k}\right) \sqrt{T}\left(\widehat{\gamma}_{T}-\gamma_{0}\right),
\end{aligned}
$$

where $\widetilde{\gamma}$ denotes a mean value on the line segment connecting $\gamma_{0}$ and $\widehat{\gamma}_{T} .{ }^{6}$ The correct specification of the conditional median turns the first term in the RHS of Equation (3.6) into a martingale difference sequence. A central limit theorem applies. To be more precise, we have

$$
\left(\boldsymbol{R}^{\prime} \overline{\boldsymbol{V}}_{T} \boldsymbol{R}\right)^{-1 / 2} \frac{1}{\sqrt{T}} \sum_{t=1}^{T} \sum_{k=1}^{K} \boldsymbol{R}_{k}^{\prime} \boldsymbol{z}_{t-1} \operatorname{sgn}\left(u_{k t}\right) \stackrel{d}{\longrightarrow} \mathrm{N}\left(\mathbf{0}, \boldsymbol{I}_{M}\right)
$$

The matrix appearing in the standardization of the random vector equals

$$
\overline{\boldsymbol{V}}_{T}=\frac{1}{T} \sum_{t=1}^{T} \sum_{k_{1}=1}^{K} \sum_{k_{2}=1}^{K} \operatorname{sgn}\left(u_{k_{1}}\right) \operatorname{sgn}\left(u_{k_{2} t}\right) \boldsymbol{z}_{t-1} \boldsymbol{z}_{t-1}^{\prime}=\frac{1}{T} \sum_{t=1}^{T} \mathrm{E}\left(\boldsymbol{\Psi}_{t} \otimes \boldsymbol{z}_{t-1} \boldsymbol{z}_{t-1}^{\prime}\right) .
$$

\footnotetext{
${ }^{5}$ These 'derivatives' are intuitive. $|x|$ has slope -1 for negative $x$ and slope +1 for positive $x$. Related to the second derivative, we consider the function $y=\operatorname{sgn}(x)$. The sign function is constant except for the abrupt increase of two at the origin. The linear approximation $\Delta y \approx \frac{\partial y}{\partial x} \Delta x$ suggest a spike of infinitesimal width and infinite height yet with an area of two underneath. This indeed describes the graph of twice the Dirac delta function.

${ }^{6}$ The mean value theorem is applied to every element of the vector. The mean values themselves will therefore differ for each component of the vector. We deliberately commit a minor abuse of notation to ease the representation of Equation (3.6).
} 
The importance of Assumption 3.7(a) is now easily understood because it prevents the asymptotic distribution from being singular.

Assumption 3.7(b) is likewise needed to rule out an asymptotic singularity. This assumption relates to the term in brackets in Equation (3.6). With a uniform LLN and Theorem 3.1 in the back of our minds, it is plausible that ${ }^{7}$

$$
\frac{2}{T} \sum_{t=1}^{T} \sum_{k=1}^{K} \delta\left(y_{k t}-\boldsymbol{z}_{t-1}^{\prime}\left(\boldsymbol{R}_{k} \widetilde{\gamma}_{T}+\boldsymbol{r}_{k}\right)\right) \boldsymbol{R}_{k}^{\prime} \boldsymbol{z}_{t-1} \boldsymbol{z}_{t-1}^{\prime} \boldsymbol{R}_{k}-\boldsymbol{R}^{\prime}\left(\frac{2}{T} \sum_{t=1}^{T} \mathrm{E}\left(\boldsymbol{\Xi}_{t} \otimes \boldsymbol{z}_{t-1} \boldsymbol{z}_{t-1}^{\prime}\right)\right) \boldsymbol{R} \stackrel{p}{\longrightarrow} \mathbf{O}
$$

The following asymptotic normality theorem follows after combining Equation (3.6), (3.7) and (3.9).

Theorem 3.2 (Asymptotic Normality) Suppose that Assumptions 1-7 hold. Then,

$$
\left(\boldsymbol{R}^{\prime} \overline{\boldsymbol{V}}_{T} \boldsymbol{R}\right)^{-1 / 2} \boldsymbol{R}^{\prime} \overline{\boldsymbol{H}}_{T} \boldsymbol{R} \sqrt{T}\left(\widehat{\gamma}_{T}-\gamma_{0}\right) \stackrel{d}{\longrightarrow} \mathrm{N}\left(\mathbf{0}, \boldsymbol{I}_{M}\right)
$$

Remark 3.3 A rigorous proof based on Huber (1967) leads to the same end result as in Theorem 3.2. There are three main differences with the intuitive/sketchy proof of the last several pages. Firstly, the first order condition of Equation (3.5) does not hold, but one can show that $-\frac{1}{T} \sum_{t=1}^{T} \sum_{k=1}^{K} \boldsymbol{R}_{k}^{\prime} \boldsymbol{z}_{t-1} \operatorname{sgn}\left(y_{k t}-\boldsymbol{z}_{t-1}^{\prime}\left(\boldsymbol{R}_{k} \widehat{\gamma}_{T}+\boldsymbol{r}_{k}\right)\right)=o_{p}\left(T^{-1 / 2}\right)$. The first order condition therefore holds asymptotically and the convergence rate is fast enough to allow a mean value expansion. Secondly, the approach of Huber (1967) reverses the order of the mean value expansion and uniform LLN. By first considering expectations one arrives at a continuously differentiable objective function which allows for a valid application of the mean value theorem. Finally, no generalized functions are used in the formal proof. The details of the full proof of Theorem 3.2 can be found in Appendix 3.A.3.

As a special case of Theorem 3.2 we consider the situation in which (1) the innovations are independent and identically distributed; and (2) no restrictions are imposed. Note

\footnotetext{
${ }^{7}$ The uniform LLN and consistency suggest the limit $\frac{2}{T} \sum_{t=1}^{T} \sum_{k=1}^{K} \mathrm{E}\left(\delta\left(u_{k t}\right) \boldsymbol{R}_{k}^{\prime} \boldsymbol{z}_{t-1} \boldsymbol{z}_{t-1}^{\prime} \boldsymbol{R}_{k}\right)$. And we rely on $\mathrm{E}\left(\delta\left(u_{k t}\right) \boldsymbol{R}_{k}^{\prime} \boldsymbol{z}_{t-1} \boldsymbol{z}_{t-1}^{\prime} \boldsymbol{R}_{k}\right)=\mathrm{E}\left(\int \delta(x) f_{k, t}(x) d x \boldsymbol{R}_{k}^{\prime} \boldsymbol{z}_{t-1} \boldsymbol{z}_{t-1}^{\prime} \boldsymbol{R}_{k}\right)=\mathrm{E}\left(f_{k, t}(0) \boldsymbol{R}_{k}^{\prime} \boldsymbol{z}_{t-1} \boldsymbol{z}_{t-1}^{\prime} \boldsymbol{R}_{k}\right)$, using the law of iterated expectations and the aforementioned property of the Dirac delta function.
} 
that the i.i.d. assumption together with the stability condition implies that the vector autoregression is strictly stationary.

Corollary 3.1 Assume that Assumptions 1-7 are valid, innovations are i.i.d., and the absence of linear restrictions (i.e. $\boldsymbol{R}=\boldsymbol{I}_{K(K p+1)}$ and $\boldsymbol{r}=\mathbf{0}$ ), then

$$
\sqrt{T}\left(\widehat{\gamma}_{T}-\gamma_{0}\right) \stackrel{d}{\longrightarrow} \mathrm{N}\left(\mathbf{0}, \frac{1}{4} \boldsymbol{\Xi}^{-1} \boldsymbol{\Psi} \boldsymbol{\Xi}^{-1} \otimes\left[E\left(z_{t-1} z_{t-1}^{\prime}\right)\right]^{-1}\right)
$$

with $\boldsymbol{\Psi}=\operatorname{diag}\left(f_{1}(0), f_{2}(0), \ldots, f_{K}(0)\right)$ and the $\left(k_{1}, k_{2}\right)$ 'th element of $\boldsymbol{\Xi}$ given by $\boldsymbol{\Xi}_{k_{1} k_{2}}=$ $\mathrm{E}\left(\operatorname{sgn}\left(u_{k_{1}, t}\right) \operatorname{sgn}\left(u_{k_{2}, t}\right)\right)$ being the $\left(k_{1}, k_{2}\right)$ 'th element of $\boldsymbol{\Xi}$.

Remark 3.4 The limiting distribution in Corollary 3.1 reveals an interesting analogy to the least squares estimator, $\sqrt{T}\left(\widehat{\gamma}_{T}-\gamma_{0}\right) \stackrel{d}{\longrightarrow} \mathrm{N}\left(\mathbf{0}, \Sigma_{u} \otimes\left[E\left(z_{t-1} z_{t-1}^{\prime}\right)\right]^{-1}\right)\left(\Sigma_{u}\right.$ denotes the covariance matrix of the error terms, see Lütkepohl (2005)). In both cases, the asymptotic covariance matrix takes the form of a kronecker product in which one part depends solely on the properties of the innovations, and the other part equals $\left[E\left(z_{t-1} z_{t-1}^{\prime}\right)\right]^{-1}$. Least squares estimation assumes a correct specification of the conditional means. The natural measure for the variation in a collection of means is the covariance matrix and it is precisely this covariance matrix $\boldsymbol{\Sigma}_{u}$ that enters the limiting distribution. Similarly, we could expect the expression $\frac{1}{4} \boldsymbol{\Xi}^{-1} \boldsymbol{\Psi} \boldsymbol{\Xi}^{-1}$ because Rao (1988) has shown that this is the relevant dispersion measure for a vector of medians.

Remark 3.5 It is well-known since the result by Zellner (1962) that the OLS and GLS estimator coincide if the regressors are the same in every equation. A similar result is valid for LAD estimation. Jun and Pinkse (2009) propose an GMM-based efficient semiparametric estimator for seemingly unrelated quantile regressions. Their estimator provides an efficiency gain over the equation-by-equation estimator unless the regressors are independent of the innovations and the regressors are the same in every equation. In the present setup this would translate to $\boldsymbol{u}_{t}$ being independent of $\boldsymbol{z}_{t-1}$ and the use of the same vector $z_{t-1}$ as the regressors in every equation. Efficiency gains might still be possible in the presence of cross-equation restrictions. However, the solution algorithm proposed by Jun and Pinkse (2009) does not allow for these cross-equation restrictions so a generalization of their method seems difficult. We leave this issue for future research.

The asymptotic normality results appearing in Theorem 2 and Corollary 3.1 are of little relevance in the absence of consistent estimators for the matrices $\overline{\boldsymbol{V}}_{T}$ and $\overline{\boldsymbol{H}}_{T}$. The- 
orem 3 provides these consistent estimators and as such allows for the construction of confidence intervals and hypothesis tests (see Section 3.2.2). The estimator for $\overline{\boldsymbol{V}}_{T}$ is straightforward. With the following definition of the LAD residuals, $\hat{u}_{k t}=y_{k t}-\boldsymbol{z}_{t-1}^{\prime}\left(\boldsymbol{R}_{k} \widehat{\gamma}_{T}+\boldsymbol{r}_{k}\right)$, Equation (3.8) suggests

$$
\widehat{\boldsymbol{V}}_{T}=\frac{1}{T} \sum_{t=1}^{T} \sum_{k_{1}=1}^{K} \sum_{k_{2}=1}^{K} \operatorname{sgn}\left(\hat{u}_{k_{1}}\right) \operatorname{sgn}\left(\hat{u}_{k_{2} t}\right) \boldsymbol{R}_{k_{1}}^{\prime} \boldsymbol{z}_{t-1} \boldsymbol{z}_{t-1}^{\prime} \boldsymbol{R}_{k_{2}} .
$$

The consistent estimation of $\overline{\boldsymbol{H}}_{T}$ is toughened by the presence of the conditional densities evaluated at zero. Several methods have been proposed in the literature to either estimate or circumvent the estimation of this density. Three popular methods and a nonexhaustive list of related references are: (1) order statistics (e.g. Hall and Sheather (1988) and Koenker and Xiao (2006)); (2) sub- or resampling methods (e.g. Chernozhukov and Fernández-Val (2005) and Xiao (2009)); or (3) kernel estimation (e.g. Powell (1984), Weiss (1991), Engle and Manganelli (2004) and White et al. (2015)). An example of the approach based on order statistics is the Siddiqui-Bloch-Gastwirth estimator, which uses a linear combination of two order statistics to estimate $1 / f(0)$. The sub- or resampling methods estimate the parameters on a large number of either subsamples of the actual data or bootstrap samples. The sample variance of this collection of parameter estimates is an immediate proxy of the asymptotic variance. The density estimation step is thereby bypassed. We will rely on kernel estimation, and define the estimator,

$$
\widehat{\boldsymbol{H}}_{T}=\frac{1}{\hat{c}_{T} T} \sum_{t=1}^{T} \sum_{k=1}^{K} \mathbb{1}_{\left\{\left|\hat{u}_{k t}\right|<\hat{c}_{k, T}\right\}} \boldsymbol{R}_{k}^{\prime} \boldsymbol{z}_{t-1} \boldsymbol{z}_{t-1}^{\prime} \boldsymbol{R}_{k}
$$

The bandwidth parameters $\hat{c}_{k, T}(k=1,2, \ldots, K)$ appearing in the kernel estimator should satisfy the following assumption.

Assumption 3.8 (Bandwidth Conditions) For every $k$, the stochastic bandwidth sequence $\left\{\hat{c}_{k, T}\right\}$ is strictly positive and there exists a non-stochastic sequence $\left\{c_{k, T}\right\}$ such that (a) $\hat{c}_{k, T} / c_{k, T} \stackrel{p}{\longrightarrow} 1 ;(b) c_{k, T}=o(1) ;$ and $(c) c_{k, T}^{-1}=o\left(T^{1 / 2}\right)$.

Assumption 3.8(a) is surely satisfied if we would start with a non-stochastic choice of the bandwidth. This assumption can be regarded as a practical convenience. The optimal bandwidth choice ${ }^{8}$ depends on curvature of the density itself. If one assumes a particular

\footnotetext{
${ }^{8} \mathrm{~A}$ common measure of performance is the mean integrated squared error. The statement 'optimal bandwidth' and the related discussion should be understood as the bandwidth which minimizes the mean integrated squared error, see Silverman (1986).
} 
reference distribution (such as the Gaussian), then one typically requires some measure of the dispersion of the density. The allowed stochastic nature of $\hat{c}_{k, T}$, hence Assumption 3.8(a), is at this point advantageous because it allows dispersion estimates to enter the bandwidth choice. Variation in the dispersion among the cross-sectional units motivates the use of different bandwidths for the various cross-sections.

The bandwidth rates are constrained by Assumptions 3.8(b) and 3.8(c). The bandwidth should shrink to zero, as stipulated by Assumption 3.8(b), to ensure that we obtain the density at zero and not a smoothed version thereof. On the other hand, Assumption 3.8(c) limits the rate at which this convergence to zero occurs. The rationale is that the expected number of residuals falling in the interval $\left[-\hat{c}_{k, T}, \hat{c}_{k, T}\right]$ should still grow with the sample size if consistency is to be obtained.

Theorem 3.3 (Consistent Estimation of $\overline{\boldsymbol{V}}_{T}$ and $\overline{\boldsymbol{H}}_{T}$ ) For the estimators $\widehat{\boldsymbol{V}}_{T}$ and $\widehat{\boldsymbol{H}}_{T}$ defined by Equations (3.10) and (3.11), we have

(a) $\left\|\widehat{\boldsymbol{V}}_{T}-\overline{\boldsymbol{V}}_{T}\right\|=o_{p}(1)$, if Assumptions 1-7 hold.

(b) $\left\|\widehat{\boldsymbol{H}}_{T}-\overline{\boldsymbol{H}}_{T}\right\|=o_{p}(1)$, if Assumptions 1-8 hold.

\subsubsection{Hypothesis Testing}

In the previous section we incorporated the linear constraints directly into the parameter vector by writing $\boldsymbol{b}=\boldsymbol{R} \gamma+\boldsymbol{r}$. This representation expresses the $K(K p+1)$-dimensional vector $\boldsymbol{b}$ in terms of the vector $\gamma$ with dimension $M \leq K(K p+1)$. There are apparently $J=K(K p+1)-M$ restrictions that account for this reduction in dimensionality. We can express the same set of linear restrictions as $\boldsymbol{C} \boldsymbol{b}=\boldsymbol{c}$, with $\boldsymbol{C}$ being a $(J \times K(K p+1))$ matrix and $c$ denoting a vector of size $J$. These two representations are equivalent, ${ }^{9}$ but the latter is more appropriate to convey the results on hypothesis testing.

We will discuss the Wald, the Score, and the Likelihood ratio (LR) tests. Strictly speaking, these tests require us to specify the (conditional) likelihood. In the current vector autoregressive setup we would have to assign distributions to the innovations and

\footnotetext{
${ }^{9}$ For given $\boldsymbol{C} \boldsymbol{b}=\boldsymbol{c}$, we assume that the first $J$ columns of $\boldsymbol{C}$ form an invertible matrix $\boldsymbol{C}_{1}$ (otherwise permute columns to make this hold). Subsequently partition the equation accordingly, i.e. [ $\left.\boldsymbol{C}_{1} \boldsymbol{C}_{2}\right]\left[\begin{array}{l}\boldsymbol{b}_{1} \\ \boldsymbol{b}_{2}\end{array}\right]=\left[\begin{array}{l}\boldsymbol{c}_{1} \\ \boldsymbol{c}_{2}\end{array}\right]$. The first set of equations gives $\boldsymbol{b}_{1}=-\boldsymbol{C}_{1}^{-1} \boldsymbol{C}_{2} \boldsymbol{b}_{2}+\boldsymbol{C}_{1}^{-1} \boldsymbol{c}_{1}$ and allows us to write $\boldsymbol{b}=\left[\begin{array}{c}-\boldsymbol{C}_{1}^{-1} \boldsymbol{C}_{2} \\ \boldsymbol{I}_{M}\end{array}\right] \boldsymbol{b}_{2}+\left[\begin{array}{c}\boldsymbol{C}_{1}^{-1} \boldsymbol{c}_{1} \\ \mathbf{0}\end{array}\right]$ which is of the form $\boldsymbol{b}=\boldsymbol{R} \boldsymbol{\gamma}+\boldsymbol{r}$.

Now let $\boldsymbol{b}=\boldsymbol{R} \gamma+\boldsymbol{r}$ be given. We note that the matrix $\boldsymbol{R}$ has rank $M$ so there exist $J$ linearly independent vectors $\left\{\boldsymbol{x}_{1}, \boldsymbol{x}_{2}, \ldots, \boldsymbol{x}_{J}\right\}$ of length $K(K p+1)$ such that $\boldsymbol{x}_{i}^{\prime} \boldsymbol{R}=\mathbf{0}$. Now construct $\boldsymbol{C}$ as the matrix having these $\boldsymbol{x}_{i}$ 's as rows, then $\boldsymbol{C b}=\boldsymbol{c}$ with $\boldsymbol{c}=\boldsymbol{C r}$.
} 
condition on the first $p$ observations. These distributions on the components of the innovations $\boldsymbol{u}_{t}$ would be mutually independent and Laplace distributed. However, the specific distribution has no influence on the asymptotic properties of the test statistics as long as Assumptions 1-7 remain valid. In this sense it might be better to add the adjective 'quasi' to the names of these test, e.g. the quasi-Wald test. The same situation is also encountered in least squares estimation where estimators and their related test statistics can be embedded in the likelihood framework if one assumes Gaussian innovations. The testing approach for absolute deviations was pioneered by Koenker and Bassett (1982) and later extended by Weiss (1991) to allow for dependency. These approaches were based on univariate series. We will provide results on the multivariate case.

The Wald test is based on the unrestricted estimator, and measures the distance between the unrestricted parameter estimate and the hypothesized parameters under the null. We denote the unrestricted parameter estimate by $\widehat{\boldsymbol{b}}_{T}^{U}$, and arrive at the following result.

Theorem 3.4 (Wald Test) Under Assumption 1-8, the Wald test statistic, $W_{T}=T\left(\widehat{C b}_{T}^{U}-\right.$ $c)^{\prime}\left[\boldsymbol{C} \widehat{\boldsymbol{H}}_{T}^{-1} \widehat{\boldsymbol{V}}_{T} \widehat{\boldsymbol{H}}_{T}^{-1} \boldsymbol{C}^{\prime}\right]^{-1}\left(\widehat{C \boldsymbol{b}}_{T}^{U}-\boldsymbol{c}\right)$, has a chi-squared limitting distribution with $\mathrm{J}$ degrees of freedom.

Although the objective function is not differentiable, one can still construct a scoretype test statistic with the usual interpretation. Let us briefly consider the least squares framework. The least squares estimator minimizes the squared residuals, or equivalently sets the derivative of the squared residuals w.r.t. the parameters to zero. This derivative is called the score, and setting it equal to zero provides the normal equations. A similar first order condition is encountered in Equation (3.5) (or in Equation (3.31) of the formal proof in the Appendix). These equations state that there is a vector quantity that vanishes asymptotically, and therefore has an interpretation similar to the score. Let $\boldsymbol{E}_{k}$ denote the set of rows of the identity matrix belonging to the cross-section $k$, i.e. the matrices $\boldsymbol{R}_{k}$ which would be implied if we would take $\boldsymbol{R}=\boldsymbol{I}_{K(K p+1)}$, and define

$$
\begin{aligned}
\boldsymbol{g}_{T}(\boldsymbol{b}) & =-\frac{1}{\sqrt{T}} \sum_{t=1}^{T} \sum_{k=1}^{K} \boldsymbol{E}_{k}^{\prime} \boldsymbol{z}_{t-1} \operatorname{sgn}\left(y_{k t}-\boldsymbol{z}_{t-1}^{\prime} \boldsymbol{E}_{k} \boldsymbol{b}\right) \\
& =-\frac{1}{\sqrt{T}} \sum_{t=1}^{T}\left(\operatorname{sgn}\left(\boldsymbol{y}_{t}-\left(\boldsymbol{I}_{k} \otimes \boldsymbol{z}_{t-1}^{\prime}\right) \boldsymbol{b}\right) \otimes \boldsymbol{I}_{K p+1}\right) \boldsymbol{z}_{t-1} .
\end{aligned}
$$

Note that the definition of $\boldsymbol{g}_{T}(\boldsymbol{b})$ originates from Equation (3.5) where the matrices $\boldsymbol{R}_{k}$ denoted columns of the identity matrix. That is, as in the least squares case the score 
is computed under the model without any restrictions on the parameters. The following Score test statistic is based on $\boldsymbol{g}_{T}(\boldsymbol{b})$, and the restricted estimator $\widehat{\boldsymbol{b}}_{T}^{R}$ (i.e. the estimator satisfying $\widehat{C \boldsymbol{b}}_{T}^{R}=\boldsymbol{c}$ ).

Theorem 3.5 (Score Test) Under Assumption 1-8, the Score test statistic,

$$
S_{T}=\boldsymbol{g}_{T}\left(\widehat{\boldsymbol{b}}_{T}^{R}\right)^{\prime} \widehat{\boldsymbol{H}}_{T}^{-1} \boldsymbol{C}^{\prime}\left[\boldsymbol{C} \widehat{\boldsymbol{H}}_{T}^{-1} \widehat{\boldsymbol{V}}_{T} \widehat{\boldsymbol{H}}_{T}^{-1} \boldsymbol{C}^{\prime}\right]^{-1} \boldsymbol{C} \widehat{\boldsymbol{H}}_{T}^{-1} \boldsymbol{g}_{T}\left(\widehat{\boldsymbol{b}}_{T}^{R}\right)
$$

has a chi-squared limitting distribution with J degrees of freedom.

The LR test is based on the objective function itself. Based on Equation (3.3) we denote the objective function by $V(\boldsymbol{b})=\sum_{t=1}^{T} \sum_{k=1}^{K}\left|y_{k t}-\boldsymbol{z}_{t-1}^{\prime} \boldsymbol{E}_{k} \boldsymbol{b}\right| . V(\boldsymbol{b})$ is thus simply the sum of absolute errors. The Likelihood Ratio test statistic is now $L R_{T}=V\left(\widehat{\boldsymbol{b}}_{T}^{R}\right)-V\left(\widehat{\boldsymbol{b}}_{T}^{U}\right)$.

Theorem 3.6 (Likelihood Ratio Test) Under Assumption 1-7, the LR test statistic,

$$
L R_{T}=V\left(\widehat{\boldsymbol{b}}_{T}^{R}\right)-V\left(\widehat{\boldsymbol{b}}_{T}^{U}\right)
$$

has the same limiting distribution as the non-pivotal quantity

$$
\frac{1}{2} \boldsymbol{g}_{T}^{\prime}\left(\boldsymbol{b}_{0}\right) \overline{\boldsymbol{H}}_{T}^{-1} \boldsymbol{C}^{\prime}\left[\boldsymbol{C} \overline{\boldsymbol{H}}_{T}^{-1} \boldsymbol{C}^{\prime}\right]^{-1} \boldsymbol{C} \overline{\boldsymbol{H}}_{T}^{-1} \boldsymbol{g}_{T}\left(\boldsymbol{b}_{0}\right)
$$

Remark 3.6 Theorems 3.4-3.6 clearly indicate that the Wald, Score and LR test do not have the same limiting distribution. This seemingly contradicts the result by Engle (1984) that these three tests have the same asymptotic properties if the objective function has a quadratic approximation asymptotically. However, one of the necessary conditions for this result is not fulfilled. The expected outer product of the score vector with itself and the Hessian matrix do not converge to the same matrix (i.e. the matrices $\overline{\boldsymbol{V}}_{T}$ and $\overline{\boldsymbol{H}}_{T}$ converge to different limits). Therefore, the Wald, Score, and LR test statistics need not be the same asymptotically, see condition (d) on page 797 of Engle (1984). The LR statistic is even non-pivotal. In the univariate case one can obtain an asymptotic pivot by appropriate rescaling, see Koenker and Bassett (1982). This remedy does not work in the present multivariate case because the correction is not of a simple multiplicative nature. 


\subsubsection{Determination of the Lag Order}

An important aspect of time series modeling by vector autoregressions is the determination of the lag order. Let us denote the true lag order by $p_{0}$. Two popular approaches to decide on the lag order are sequential testing and information criteria. Both methods assume that the researcher knows a $p_{\max }$ such that $p_{0} \leq p_{\max }$.

The sequential testing procedure is based on a series of tests with the null hypothesis being that all the elements of the coefficient matrix in front of the $p$ 'th lag are equal to zero. The procedure stops as soon as a null hypothesis is rejected. This sequential testing procedure is usually based on a least squares approach, but one can use the test statistics derived in Section 3.2.2 as an $L_{1}$ counterpart.

It remains to be seen whether we can also obtain information criteria that are based on least absolute deviations. The general form of information criteria is

$$
\operatorname{Cr}(p)=-2 \log \left(L\left(\widehat{\boldsymbol{b}}_{T}^{(p)}\right)\right)+k c_{T}
$$

with $k=\operatorname{dim}(\boldsymbol{b})$. The first term is a measure a fit, i.e. the (quasi-)likelihood evaluated at parameter estimates from a $\operatorname{VAR}(p)$. The function $c_{T}$ penalizes for the use of parameters. Popular choices for $c_{T}$ are: (1) $c_{T}=2$ for the AIC criterion, (2) $c_{T}=\log (T)$ for the BIC criterion, and (3) $c_{T}=2 \log (\log (T))$ for the HQ criterion. The log-likelihood under a (quasi) Gaussian framework is given by $-\frac{T}{2} \log \left(\widehat{\Sigma}_{u}^{(p)}\right)$ with $\widehat{\Sigma}_{u}^{(p)}$ the estimate of the error covariance matrix. ${ }^{10}$ In our $L_{1}$ likelihood framework of Laplace random variables (i.i.d. in both time and cross-section), the log-likelihood contribution is $-K T \log \left(F\left(\widehat{\boldsymbol{b}}_{T}^{(p)}\right)\right)$, with $F\left(\widehat{\boldsymbol{b}}_{T}^{(p)}\right)=\frac{1}{K T} \sum_{t=1}^{T} \sum_{k=1}^{K}\left|y_{k t}-\boldsymbol{z}_{t-1}^{\prime} \boldsymbol{E}_{k} \widehat{\boldsymbol{b}}_{T}^{(p)}\right|$. The following theorem comments on the consistency of the model selection procedure.

Theorem 3.7 (Consistent Selection of the Lag Order) Assume that $p_{0}<p_{\max }$, and let $\hat{p}$ denote the lag order of the model with the smallest information criteria. If $c_{T} \rightarrow \infty$ and $c_{T} / T \rightarrow 0$, then $\operatorname{Pr}\left(\hat{p}=p_{0}\right) \rightarrow 1$ as $T \rightarrow \infty$.

The previous theorem indicates that the BIC penalty provides consistent model selection. For illustration and later reference, we explicitly list the LAD and OLS the informa-

\footnotetext{
${ }^{10}$ We ignore additive constants because they have no effect on the selection procedure.
} 
tion criteria based on the BIC penalty

$$
\begin{aligned}
& \mathrm{BIC}_{L A D}=2 K T \log \left(F\left(\widehat{\boldsymbol{b}}_{T}^{(p)}\right)\right)+K(1+K p) \log (T), \\
& \mathrm{BIC}_{O L S}=\log \left(\widehat{\Sigma}_{u}^{(p)}\right)+K(1+K p) \log (T) .
\end{aligned}
$$

\subsection{Simulation Results}

A Monte Carlo study was conducted to gain insight into the performance of the estimator, the test statistics, and the information criteria. The simulations are based on the following data generating process (DGP),

$$
\boldsymbol{y}_{t}=\left[\begin{array}{l}
0.02 \\
0.03
\end{array}\right]+\left[\begin{array}{ll}
0.5 & 0.1 \\
0.4 & 0.5
\end{array}\right] \boldsymbol{y}_{t-1}+\left[\begin{array}{cc}
\alpha & \alpha \\
0.25 & 0
\end{array}\right] \boldsymbol{y}_{t-2}+L \boldsymbol{u}_{t}, \quad L=\left[\begin{array}{cc}
0.3 & 0 \\
0 & 0.2
\end{array}\right]
$$

We start with $\alpha=0.25$, a parameter specification due to Lütkepohl (2005). ${ }^{11}$ The elements in the innovation vector $\boldsymbol{u}_{t}$ are generated i.i.d. from various distributions. The considered distributions for $u_{k t}$ are: (1) $\mathrm{N}(0,1)$ variates; (2) Laplace distributed innovations with unit variance; (3) Student-t random variables with degrees of freedom being either 3, 4, 5, 10 or 20; (4) contaminated Gaussian innovations with various degrees of pollution. The latter setting is inspired by the robustness literature, e.g. Huber and Ronchetti (2009). The cumulative distribution function of $u_{k t}$ is specified as $F(x)=(1-\epsilon) \Phi(x)+\epsilon \Phi(x / 4)$, where $\Phi$ denotes the CDP of a standard normal random variable. This innovation distribution is a mixture of two normal distributions where with probability $\epsilon$ we encounter an innovation that has a four times larger standard deviation. The contamination is component wise. This implies that $u_{1 t}$ can be a draw from the narrow distribution whereas $u_{2 t}$ might originate from the wide distribution. A discussion on various outlier generating processes for multivariate settings can be found in Alqallaf et al. (2009).

Some general remarks concerning the simulation setup are as follows. The largest absolute eigenvalue of the companion matrix associated with the VAR in Equation (3.15) is 0.769 . The autoregressive process is thus stable. A presample of 1,000 observations was used to remove possible influences of initial values. All results are based on 100,000 Monte Carlo replications.

\footnotetext{
${ }^{11}$ This DGP is used in Lütkepohl (2005) in both theoretical examples and Monte Carlo Simulations. The related sections are 2.1.1, 3.2.4, 3.5.4, 4.2.1. and 4.3.4. Corresponding results obtained from least squares estimation are therefore easily available.
} 
Assumption 3.8 only puts rate restrictions on the bandwidth, and hence does not specify the exact choice. We compute the bandwidth related to the $k^{\prime}$ th cross-section as

$$
\begin{aligned}
\hat{c}_{k, T} & =\hat{\kappa}_{k, T}\left[\Phi^{-1}\left(1 / 2+h_{T}\right)-\Phi^{-1}\left(1 / 2-h_{T}\right)\right] \\
h_{T} & =T^{-1 / 3}\left(\Phi^{-1}(1-0.05 / 2)\right)^{2 / 3}\left(\frac{1.5\left(\phi\left(\Phi^{-1}(1 / 2)\right)\right)^{2}}{2\left(\Phi^{-1}(1 / 2)\right)^{2}+1}\right)^{1 / 3}
\end{aligned}
$$

where $\Phi(z)$ and $\phi(z)$ are, respectively, the cumulative distristribution function and probability denstity function of a standard normal random variable. $\hat{\kappa}_{k, T}$ is a measure of scale and is taken equal to the median absolute deviation of the residuals in the $k$ 'th crosssection. This procedure of bandwidth selection dates back to Koenker (2005), and was also used by White et al. (2015).

The first evidence of the appropriateness of the asymptotic approximation as given by Theorem 3.2 is displayed in Figure 3.1. This figure displays the histograms of the Monte Carlo replicates for the constrained and unconstrained estimator. The unconstrained estimator estimates all ten parameters, whereas the constrained estimator correctly puts zero constraints on the coefficients that are zero in the DGP. The histograms for the coefficient $\hat{a}_{12,1}$ are displayed. The true value of this coefficient is 0.1 and the histograms are indeed centered around this value. The Gaussian approximation seems appropriate at a sample size of $T=100$. We can also see how the constrained estimator reduces parameter uncertainty because fewer parameters are estimated.

Tables 3.1-3.6 list the empirical MSE of the least squares and the least absolute deviation estimators. Results are reported for the two intercept coefficients and the autoregressive parameters encountered in the first cross-section. The corresponding true parameter values are $[0.02,0.03,0.5,0.1,0,0]$. The behavior as observed in Tables 3.1-3.3 is expected. For standard normal (Laplace) errors, the OLS (LAD) estimator is the maximum likelihood estimator and performs best. The Student-t distribution is strongly leptokurtic for small degrees of freedom but starts to behave more and more like a Gaussian distribution as the degrees of freedom increase. This is also reflected in the tables. The LAD estimator performs well for small degrees of freedom, but compares less favorable to OLS as the innovation's distribution starts to resemble the Gaussian distribution. Results on the mixture innovation distribution are reported in Table 3.4-3.6. The percentage of outliers, $\epsilon$, varies from $1 \%$ to $10 \%$. The simulation outcomes suggest that the LAD estimator 
should be preferred if more than $3 \%$ of the innovations can be considered outliers. ${ }^{12}$ Note that these previous observations hold irrespective of the sample size $(T=100, T=250$ or $T=500)$ and irrespective of the specific parameter.

Our Monte Carlo experiment on hypothesis testing relates to the parameter $\alpha$, i.e. we would like to test whether a second lag is needed in the first equation. The choice $\alpha=0$ allows us to assess the size of the test, and we take $\alpha=-0.1$ and $\alpha=-0.2$ to investigate the power. ${ }^{13}$ The Tables 3.7-3.12 gather the results on empirical size and power. The general messages conveyed by these tables are now listed. Firstly, the size of the test is close to the nominal level and improves with sample size. Secondly, the pattern in the power of the tests is comparable to the findings for the empirical MSE. The OLS-based tests have higher power if the distribution is close to Gaussian (Student- $t$ innovations with high degrees of freedom, or low fractions of contamination), whereas LAD-based tests obtain higher power for leptokurtic distributions or higher fractions of contamination. Thirdly, the size of the quasi-Laplacian LM test is better controlled than its Wald counterpart. If an LAD-type of test is used, then we would recommend the LM test because its empirical size is closest to the nominal level.

Simulation results on the information criterion are reported in Tables 3.13 and 3.14 for a sample size of $T=50$. We set $\alpha=0$ and replace the value 0.25 in the DGP of Equation (3.15) by zero. The true lag order of the VAR is thus $p_{0}=1$. The aforementioned tables show the selection frequencies for VAR models with zero up to three lags. For brevity, we only consider the BIC-type information criteria of Equation (3.14). These criteria should consistently select the lag order. This also happens because both criteria choose the true lag order over $90 \%$ of the times. The least squares based criterion marginally selects the true model more often than the LAD counterparts. Additional simulation results for sample sizes of $T=100$ and $T=150$ are reported in Appendix 3.C.2. These simulation results provide evidence that these BIC-type criteria are indeed consistent because the selection frequency of the model with a single lag is almost exactly equal to one.

\subsection{Conclusion}

In this paper we consider inference on VAR models by least absolute deviation. The estimator solves a linear program, and is therefore easy to compute. The interpretation of

\footnotetext{
${ }^{12}$ The exact turning point, here $3 \%$, depends on the two standard deviations of the normal distributions and should therefore not be regarded as a general rule.

${ }^{13}$ The variation to negative $\alpha$ was chosen to ensure stability of the VAR. The largest root in absolute value is decreases only slightly when $\alpha$ is made more negative.
} 
the LAD estimator as a quasi-maximum likelihood estimator under i.i.d. Laplacian errors leads naturally to LAD-based Wald, Lagrange Multiplier, and Likelihood Ratio test statistics. The asymptotic distribution of the first two statistics is chi-squared, whereas the Likelihood Ratio test is bounded in probability but non-pivotal. The Wald and Lagrange Multiplier tests can thus be used in the conventional way. Simulations show that these tests have good size properties in finite samples. Efficiency gains in terms of meansquared error and increased power are found if the innovations deviate from Gaussianity. The information criteria based on the Laplacian likelihood and BIC penalty perform similarly (or marginally worse) in terms of selection of the correct lag order. In general, the simulations indicate that the LAD estimator is a competitive alternative to least squares estimation unless one has a strong belief in normality of the innovations driving the vector autoregression. 


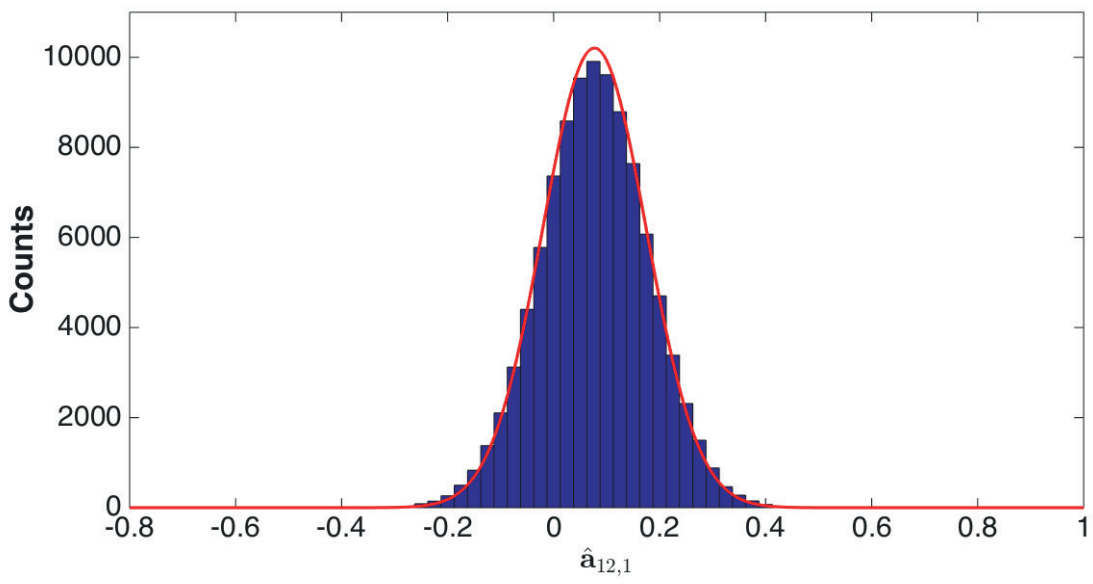

(a) Constrained

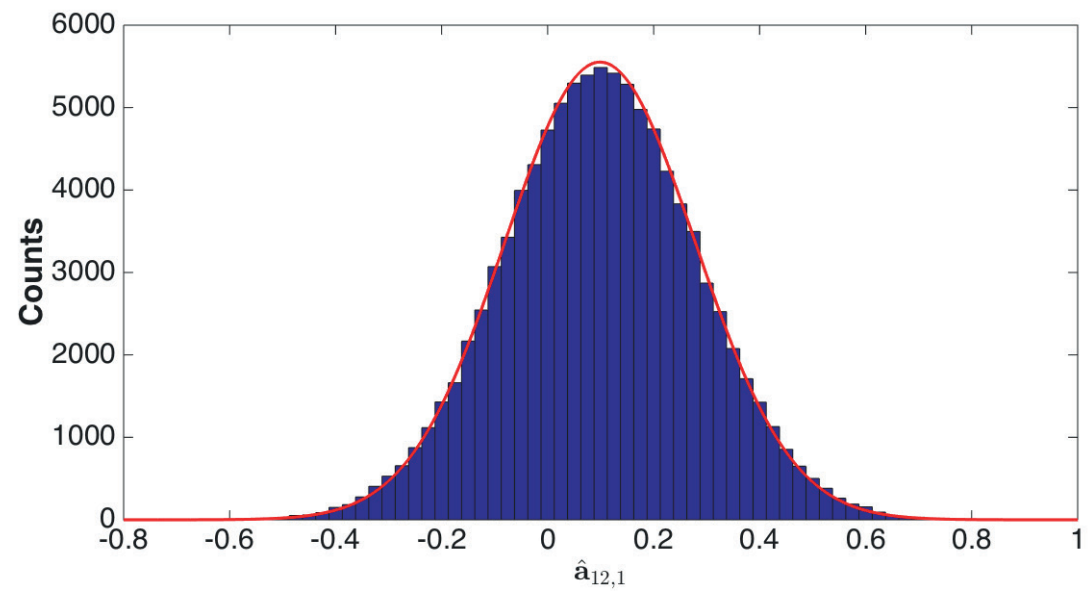

(b) Unconstrained

Figure 3.1: The finite sample distribution of the estimated coefficient $\hat{a}_{12,1}$ based on 100,000 MC replicates. The unconstrained estimator estimates all the parameters in Equation (3.15) whereas the constrained estimator uses the correct zero pattern. The sample size is $T=100$. The red line depicts the expected counts based on the asymptotic approximation. The distribution is centered around the true parameter value 0.1 and has a Gaussian form. Similar histograms for the larger sample sizes of $T=250$ and $T=500$ can be found in Appendix 3.C.1. 


\begin{tabular}{cc||c|c|c|c|c|c} 
& & $\boldsymbol{\nu}_{1}$ & $\boldsymbol{\nu}_{2}$ & $\boldsymbol{A}_{11,1}$ & $\boldsymbol{A}_{12,1}$ & $\boldsymbol{A}_{21,1}$ & $\boldsymbol{A}_{21,1}$ \\
\hline \hline \multirow{2}{*}{$\mathcal{N}(0,1)$} & LAD & 6.163 & 3.418 & 13.068 & 18.338 & 8.617 & 12.262 \\
& OLS & 5.408 & 2.787 & 10.693 & 14.659 & 6.916 & 9.847 \\
\hline \multirow{2}{*}{ Laplace } & LAD & 4.111 & 2.304 & 9.129 & 12.866 & 6.025 & 8.488 \\
& OLS & 5.419 & 2.813 & 10.579 & 14.856 & 7.008 & 9.720 \\
\hline \multirow{2}{*}{$\mathrm{t}(3)$} & LAD & 6.132 & 3.532 & 9.309 & 13.067 & 6.205 & 8.632 \\
& OLS & 8.474 & 4.496 & 10.317 & 15.344 & 7.538 & 9.468 \\
\hline \multirow{2}{*}{$\mathrm{t}(4)$} & LAD & 6.236 & 3.500 & 10.392 & 14.673 & 6.853 & 9.707 \\
& OLS & 7.239 & 3.744 & 10.486 & 15.029 & 7.109 & 9.676 \\
\hline \multirow{2}{*}{$\mathrm{t}(5)$} & LAD & 6.225 & 3.482 & 11.028 & 15.485 & 7.272 & 10.271 \\
& OLS & 6.701 & 3.453 & 10.554 & 14.811 & 7.006 & 9.746 \\
\hline \multirow{2}{*}{$\mathrm{t}(10)$} & LAD & 6.209 & 3.475 & 12.159 & 17.060 & 8.006 & 11.344 \\
& OLS & 5.924 & 3.070 & 10.630 & 14.762 & 6.931 & 9.848 \\
\hline \multirow{2}{*}{$\mathrm{t}(20)$} & LAD & 6.191 & 3.451 & 12.620 & 17.725 & 8.313 & 11.759 \\
& OLS & 5.648 & 2.921 & 10.679 & 14.715 & 6.910 & 9.826
\end{tabular}

Table 3.1: The root-mean-square error (RMSE) times 100 for several parameter estimates and various innovation distributions. The DGP is given in Equation (3.15). The sample size is $T=100$.

\begin{tabular}{cc||c|c|c|c|c|c} 
& & $\boldsymbol{\nu}_{1}$ & $\boldsymbol{\nu}_{2}$ & $\boldsymbol{A}_{11,1}$ & $\boldsymbol{A}_{12,1}$ & $\boldsymbol{A}_{21,1}$ & $\boldsymbol{A}_{21,1}$ \\
\hline \hline \multirow{2}{*}{$\mathcal{N}(0,1)$} & LAD & 3.166 & 1.908 & 8.060 & 11.449 & 5.319 & 7.659 \\
& OLS & 2.658 & 1.535 & 6.461 & 9.166 & 4.251 & 6.132 \\
\hline \multirow{2}{*}{ Laplace } & LAD & 1.956 & 1.204 & 5.213 & 7.496 & 3.479 & 4.979 \\
& OLS & 2.649 & 1.538 & 6.448 & 9.253 & 4.279 & 6.090 \\
\hline \multirow{2}{*}{$\mathrm{t}(3)$} & LAD & 3.125 & 1.935 & 5.468 & 7.816 & 3.632 & 5.173 \\
& OLS & 4.211 & 2.493 & 6.318 & 9.504 & 4.535 & 5.931 \\
\hline \multirow{2}{*}{$\mathrm{t}(4)$} & LAD & 3.131 & 1.939 & 6.224 & 8.893 & 4.150 & 5.924 \\
& OLS & 3.522 & 2.051 & 6.378 & 9.245 & 4.358 & 6.067 \\
\hline \multirow{2}{*}{$\mathrm{t}(5)$} & LAD & 3.153 & 1.930 & 6.679 & 9.508 & 4.414 & 6.339 \\
& OLS & 3.268 & 1.894 & 6.446 & 9.214 & 4.295 & 6.110 \\
\hline \multirow{2}{*}{$\mathrm{t}(10)$} & LAD & 3.143 & 1.931 & 7.438 & 10.624 & 4.923 & 7.035 \\
& OLS & 2.901 & 1.690 & 6.486 & 9.197 & 4.274 & 6.134 \\
\hline \multirow{2}{*}{$\mathrm{t}(20)$} & LAD & 3.142 & 1.916 & 7.746 & 11.085 & 5.149 & 7.346 \\
& OLS & 2.760 & 1.603 & 6.470 & 9.195 & 4.263 & 6.108
\end{tabular}

Table 3.2: Identical to Table 3.1, but for $T=250$. 


\begin{tabular}{cc||c|c|c|c|c|c} 
& & $\boldsymbol{\nu}_{1}$ & $\boldsymbol{\nu}_{2}$ & $\boldsymbol{A}_{11,1}$ & $\boldsymbol{A}_{12,1}$ & $\boldsymbol{A}_{21,1}$ & $\boldsymbol{A}_{21,1}$ \\
\hline \hline \multirow{2}{*}{$\mathcal{N}(0,1)$} & LAD & 2.042 & 1.297 & 5.634 & 8.111 & 3.783 & 5.410 \\
& OLS & 1.679 & 1.041 & 4.524 & 6.469 & 3.004 & 4.325 \\
\hline \multirow{2}{*}{ Laplace } & LAD & 1.239 & 0.791 & 3.530 & 5.061 & 2.347 & 3.363 \\
& OLS & 1.684 & 1.043 & 4.523 & 6.481 & 3.002 & 4.317 \\
\hline \multirow{2}{*}{$\mathrm{t}(3)$} & LAD & 2.041 & 1.313 & 3.741 & 5.376 & 2.491 & 3.571 \\
& OLS & 2.702 & 1.669 & 4.434 & 6.632 & 3.145 & 4.217 \\
\hline \multirow{2}{*}{$\mathrm{t}(4)$} & LAD & 2.047 & 1.313 & 4.322 & 6.193 & 2.880 & 4.137 \\
& OLS & 2.271 & 1.396 & 4.494 & 6.520 & 3.019 & 4.284 \\
\hline \multirow{2}{*}{$\mathrm{t}(5)$} & LAD & 2.054 & 1.313 & 4.604 & 6.670 & 3.084 & 4.456 \\
& OLS & 2.082 & 1.290 & 4.481 & 6.509 & 3.020 & 4.315 \\
\hline \multirow{2}{*}{$\mathrm{t}(10)$} & LAD & 2.057 & 1.308 & 5.176 & 7.472 & 3.453 & 4.950 \\
& OLS & 1.847 & 1.144 & 4.514 & 6.499 & 3.014 & 4.309 \\
\hline \multirow{2}{*}{$\mathrm{t}(20)$} & LAD & 2.056 & 1.303 & 5.444 & 7.812 & 3.612 & 5.196 \\
& OLS & 1.759 & 1.087 & 4.530 & 6.478 & 2.995 & 4.320
\end{tabular}

Table 3.3: Identical to Table 3.1, but for $T=500$.

\begin{tabular}{lc||c|c|c|c|c|c} 
& & $\boldsymbol{\nu}_{1}$ & $\boldsymbol{\nu}_{2}$ & $\boldsymbol{A}_{11,1}$ & $\boldsymbol{A}_{12,1}$ & $\boldsymbol{A}_{21,1}$ & $\boldsymbol{A}_{21,1}$ \\
\hline \hline \multirow{2}{*}{$\varepsilon=0.01$} & LAD & 6.048 & 3.402 & 12.434 & 17.551 & 8.222 & 11.663 \\
& OLS & 5.668 & 2.947 & 10.545 & 14.821 & 7.027 & 9.769 \\
\hline \multirow{2}{*}{$\varepsilon=0.02$} & LAD & 6.041 & 3.403 & 11.974 & 16.742 & 7.889 & 11.130 \\
& OLS & 5.995 & 3.126 & 10.479 & 14.900 & 7.100 & 9.647 \\
\hline \multirow{2}{*}{$\varepsilon=0.03$} & LAD & 5.980 & 3.399 & 11.515 & 16.168 & 7.582 & 10.726 \\
& OLS & 6.246 & 3.258 & 10.489 & 15.117 & 7.133 & 9.603 \\
\hline \multirow{2}{*}{$\varepsilon=0.04$} & LAD & 5.958 & 3.374 & 11.119 & 15.600 & 7.326 & 10.309 \\
& OLS & 6.505 & 3.409 & 10.389 & 15.159 & 7.212 & 9.574 \\
\hline \multirow{2}{*}{$\varepsilon=0.05$} & LAD & 5.909 & 3.398 & 10.731 & 15.070 & 7.115 & 10.025 \\
& OLS & 6.719 & 3.530 & 10.381 & 15.118 & 7.203 & 9.567 \\
\hline \multirow{2}{*}{$\varepsilon=0.06$} & LAD & 5.952 & 3.396 & 10.341 & 14.653 & 6.894 & 9.668 \\
& OLS & 7.014 & 3.646 & 10.341 & 15.219 & 7.200 & 9.500 \\
\hline \multirow{2}{*}{$\varepsilon=0.07$} & LAD & 5.935 & 3.396 & 10.153 & 14.293 & 6.708 & 9.440 \\
& OLS & 7.243 & 3.765 & 10.356 & 15.244 & 7.210 & 9.534 \\
\hline \multirow{2}{*}{$\varepsilon=0.08$} & LAD & 5.961 & 3.410 & 9.829 & 13.868 & 6.520 & 9.181 \\
& OLS & 7.450 & 3.890 & 10.289 & 15.186 & 7.224 & 9.490 \\
\hline \multirow{2}{*}{$\varepsilon=0.09$} & LAD & 5.965 & 3.453 & 9.636 & 13.610 & 6.385 & 8.980 \\
& OLS & 7.690 & 4.008 & 10.411 & 15.219 & 7.238 & 9.515 \\
\hline \multirow{2}{*}{$\varepsilon=0.10$} & LAD & 6.003 & 3.462 & 9.424 & 13.313 & 6.273 & 8.795 \\
& OLS & 7.943 & 4.138 & 10.336 & 15.268 & 7.233 & 9.531 \\
\hline \multirow{2}{*}{$3.4=$}
\end{tabular}

Table 3.4: The root-mean-square error (RMSE) times 100 for several parameter estimates and a varying percentage of outliers in the mixture distribution. The DGP is given in Equation (3.15). The sample size is $T=100$. 


\begin{tabular}{lc||c|c|c|c|c|c} 
& & $\boldsymbol{\nu}_{1}$ & $\boldsymbol{\nu}_{2}$ & $\boldsymbol{A}_{11,1}$ & $\boldsymbol{A}_{12,1}$ & $\boldsymbol{A}_{21,1}$ & $\boldsymbol{A}_{21,1}$ \\
\hline \hline \multirow{2}{*}{$\varepsilon=0.01$} & LAD & 3.122 & 1.900 & 7.624 & 10.891 & 5.078 & 7.247 \\
& OLS & 2.806 & 1.628 & 6.425 & 9.239 & 4.315 & 6.091 \\
\hline \multirow{2}{*}{$\varepsilon=0.02$} & LAD & 3.089 & 1.898 & 7.219 & 10.357 & 4.826 & 6.904 \\
& OLS & 2.943 & 1.719 & 6.412 & 9.266 & 4.344 & 6.070 \\
\hline \multirow{2}{*}{$\varepsilon=0.03$} & LAD & 3.090 & 1.892 & 6.954 & 9.896 & 4.627 & 6.594 \\
& OLS & 3.082 & 1.792 & 6.386 & 9.284 & 4.350 & 6.046 \\
\hline \multirow{2}{*}{$\varepsilon=0.04$} & LAD & 3.052 & 1.881 & 6.687 & 9.543 & 4.446 & 6.336 \\
& OLS & 3.186 & 1.861 & 6.385 & 9.326 & 4.345 & 6.035 \\
\hline \multirow{2}{*}{$\varepsilon=0.05$} & LAD & 3.074 & 1.894 & 6.441 & 9.245 & 4.280 & 6.136 \\
& OLS & 3.332 & 1.945 & 6.366 & 9.345 & 4.353 & 6.054 \\
\hline \multirow{2}{*}{$\varepsilon=0.06$} & LAD & 3.061 & 1.901 & 6.222 & 8.901 & 4.157 & 5.923 \\
& OLS & 3.462 & 2.018 & 6.389 & 9.294 & 4.362 & 6.027 \\
\hline \multirow{2}{*}{$\varepsilon=0.07$} & LAD & 3.063 & 1.906 & 6.087 & 8.687 & 4.040 & 5.754 \\
& OLS & 3.564 & 2.086 & 6.399 & 9.346 & 4.371 & 6.056 \\
\hline \multirow{2}{*}{$\varepsilon=0.08$} & LAD & 3.068 & 1.912 & 5.900 & 8.411 & 3.922 & 5.607 \\
& OLS & 3.669 & 2.142 & 6.356 & 9.283 & 4.353 & 6.043 \\
\hline \multirow{2}{*}{$\varepsilon=0.09$} & LAD & 3.080 & 1.919 & 5.766 & 8.245 & 3.844 & 5.461 \\
& OLS & 3.785 & 2.211 & 6.363 & 9.322 & 4.345 & 6.029 \\
\hline \multirow{2}{*}{$\varepsilon=0.10$} & LAD & 3.088 & 1.928 & 5.619 & 8.041 & 3.741 & 5.333 \\
& OLS & 3.888 & 2.266 & 6.361 & 9.288 & 4.358 & 6.017
\end{tabular}

Table 3.5: Identical to Table 3.4, but for $T=250$.

\begin{tabular}{lc||c|c|c|c|c|c} 
& & $\boldsymbol{\nu}_{1}$ & $\boldsymbol{\nu}_{2}$ & $\boldsymbol{A}_{11,1}$ & $\boldsymbol{A}_{12,1}$ & $\boldsymbol{A}_{21,1}$ & $\boldsymbol{A}_{21,1}$ \\
\hline \hline \multirow{2}{*}{$\varepsilon=0.01$} & LAD & 2.029 & 1.294 & 5.338 & 7.642 & 3.549 & 5.097 \\
& OLS & 1.779 & 1.106 & 4.514 & 6.500 & 3.017 & 4.295 \\
\hline \multirow{2}{*}{$\varepsilon=0.02$} & LAD & 2.006 & 1.286 & 5.053 & 7.280 & 3.360 & 4.846 \\
& OLS & 1.871 & 1.158 & 4.491 & 6.533 & 3.006 & 4.301 \\
\hline \multirow{2}{*}{$\varepsilon=0.03$} & LAD & 2.016 & 1.288 & 4.823 & 6.961 & 3.239 & 4.630 \\
& OLS & 1.966 & 1.217 & 4.482 & 6.519 & 3.049 & 4.292 \\
\hline \multirow{2}{*}{$\varepsilon=0.04$} & LAD & 2.009 & 1.287 & 4.625 & 6.669 & 3.108 & 4.434 \\
& OLS & 2.041 & 1.271 & 4.469 & 6.513 & 3.046 & 4.252 \\
\hline \multirow{2}{*}{$\varepsilon=0.05$} & LAD & 2.012 & 1.292 & 4.480 & 6.407 & 2.986 & 4.271 \\
& OLS & 2.129 & 1.315 & 4.488 & 6.526 & 3.040 & 4.273 \\
\hline \multirow{2}{*}{$\varepsilon=0.06$} & LAD & 2.009 & 1.290 & 4.343 & 6.238 & 2.896 & 4.137 \\
& OLS & 2.198 & 1.363 & 4.478 & 6.533 & 3.049 & 4.265 \\
\hline \multirow{2}{*}{$\varepsilon=0.07$} & LAD & 2.023 & 1.295 & 4.201 & 6.048 & 2.802 & 4.026 \\
& OLS & 2.282 & 1.418 & 4.481 & 6.541 & 3.028 & 4.274 \\
\hline \multirow{2}{*}{$\varepsilon=0.08$} & LAD & 2.022 & 1.304 & 4.091 & 5.917 & 2.731 & 3.903 \\
& OLS & 2.348 & 1.462 & 4.480 & 6.545 & 3.043 & 4.273 \\
\hline \multirow{2}{*}{$\varepsilon=0.09$} & LAD & 2.036 & 1.308 & 3.991 & 5.734 & 2.660 & 3.830 \\
& OLS & 2.411 & 1.500 & 4.473 & 6.529 & 3.033 & 4.286 \\
\hline \multirow{2}{*}{$\varepsilon=0.10$} & LAD & 2.042 & 1.314 & 3.889 & 5.608 & 2.608 & 3.722 \\
& OLS & 2.494 & 1.542 & 4.480 & 6.531 & 3.025 & 4.272
\end{tabular}

Table 3.6: Identical to Table 3.4, but for $T=500$. 


\begin{tabular}{cc||cc|ccc} 
& \multicolumn{1}{c||}{} & \multicolumn{2}{c|}{ LAD } & \multicolumn{3}{c}{ OLS } \\
& $\boldsymbol{\alpha}$ & Wald & LM & Wald & LM & LR \\
\hline \hline \multirow{3}{*}{$\mathcal{N}(0,1)$} & 0 & 6.30 & 4.91 & 6.07 & 5.09 & 5.56 \\
& -0.1 & 12.82 & 11.11 & 18.19 & 16.16 & 17.18 \\
& -0.2 & 29.79 & 28.01 & 47.83 & 44.71 & 46.28 \\
\hline \multirow{3}{*}{ Laplace } & 0 & 3.69 & 4.79 & 5.88 & 4.89 & 5.36 \\
& -0.1 & 13.81 & 18.38 & 17.92 & 15.97 & 16.97 \\
& -0.2 & 45.53 & 49.82 & 47.83 & 44.80 & 46.36 \\
\hline \multirow{3}{*}{$\mathrm{t}(3)$} & 0 & 6.56 & 4.80 & 5.81 & 4.91 & 5.36 \\
& -0.1 & 20.27 & 17.05 & 17.78 & 15.78 & 16.79 \\
& -0.2 & 51.69 & 47.99 & 48.41 & 45.51 & 47.00 \\
\hline \multirow{2}{*}{$\mathrm{t}(4)$} & 0 & 5.95 & 4.87 & 5.88 & 4.95 & 5.39 \\
& -0.1 & 16.13 & 14.50 & 17.65 & 15.70 & 16.70 \\
& -0.2 & 42.80 & 40.87 & 48.17 & 45.17 & 46.69 \\
\hline \multirow{2}{*}{$\mathrm{t}(5)$} & 0 & 5.89 & 4.76 & 5.97 & 5.03 & 5.50 \\
& -0.1 & 14.76 & 13.41 & 17.79 & 15.86 & 16.84 \\
& -0.2 & 38.71 & 37.51 & 48.03 & 44.89 & 46.53 \\
\hline \multirow{2}{*}{$\mathrm{t}(10)$} & 0 & 5.97 & 4.89 & 6.13 & 5.14 & 5.63 \\
& -0.1 & 13.45 & 12.09 & 17.64 & 15.70 & 16.71 \\
& -0.2 & 33.14 & 32.21 & 47.90 & 44.88 & 46.48 \\
\hline \multirow{2}{*}{$\mathrm{t}(20)$} & 0 & 6.07 & 4.87 & 6.15 & 5.14 & 5.65 \\
& -0.1 & 12.95 & 11.55 & 18.01 & 15.98 & 16.97 \\
& -0.2 & 31.45 & 30.01 & 48.19 & 45.22 & 46.75
\end{tabular}

Table 3.7: The empirical size and power (in percentages) of the test statistics for various innovation distributions. The desired nominal size is 5\%. The DGP is given in Equation (3.15). The sample size is $T=100$. 


\begin{tabular}{cc||cc|ccc} 
& \multicolumn{1}{c||}{} & \multicolumn{2}{c}{ LAD } & \multicolumn{3}{c}{ OLS } \\
& $\boldsymbol{\alpha}$ & Wald & LM & Wald & LM & LR \\
\hline \hline \multirow{2}{*}{$\mathcal{N}(0,1)$} & 0 & 5.76 & 4.68 & 5.34 & 4.95 & 5.14 \\
& -0.1 & 21.05 & 19.91 & 31.66 & 30.61 & 31.15 \\
& -0.2 & 60.66 & 61.91 & 84.06 & 83.29 & 83.68 \\
\hline \multirow{3}{*}{ Laplace } & 0 & 3.35 & 4.89 & 5.40 & 5.05 & 5.22 \\
& -0.1 & 34.55 & 41.69 & 32.15 & 31.04 & 31.62 \\
& -0.2 & 89.56 & 90.84 & 83.82 & 83.06 & 83.45 \\
\hline \multirow{3}{*}{$\mathrm{t}(3)$} & 0 & 6.87 & 4.73 & 5.23 & 4.92 & 5.08 \\
& -0.1 & 40.80 & 37.45 & 32.22 & 31.19 & 31.71 \\
& -0.2 & 88.35 & 89.16 & 82.86 & 82.15 & 82.50 \\
\hline \multirow{2}{*}{$\mathrm{t}(4)$} & 0 & 6.00 & 4.82 & 5.37 & 5.00 & 5.17 \\
& -0.1 & 31.38 & 30.51 & 31.86 & 30.79 & 31.34 \\
& -0.2 & 80.67 & 81.85 & 83.57 & 82.87 & 83.23 \\
\hline \multirow{2}{*}{$\mathrm{t}(5)$} & 0 & 5.74 & 4.80 & 5.41 & 5.05 & 5.23 \\
& -0.1 & 27.95 & 27.20 & 32.02 & 30.93 & 31.47 \\
& -0.2 & 75.96 & 77.36 & 83.87 & 83.15 & 83.50 \\
\hline \multirow{2}{*}{$\mathrm{t}(10)$} & 0 & 5.72 & 4.83 & 5.49 & 5.13 & 5.31 \\
& -0.1 & 23.72 & 22.82 & 32.13 & 30.98 & 31.56 \\
& -0.2 & 67.57 & 68.96 & 84.08 & 83.36 & 83.74 \\
\hline \multirow{2}{*}{$\mathrm{t}(20)$} & 0 & 5.76 & 4.79 & 5.46 & 5.10 & 5.29 \\
& -0.1 & 22.37 & 21.54 & 32.09 & 30.96 & 31.52 \\
& -0.2 & 63.73 & 65.02 & 84.17 & 83.46 & 83.81 \\
& & & & &
\end{tabular}

Table 3.8: Identical to Table 3.7, but for $T=250$. 


\begin{tabular}{cc||cc|ccc} 
& \multicolumn{1}{c||}{} & \multicolumn{2}{c}{ LAD } & \multicolumn{3}{c}{ OLS } \\
& $\boldsymbol{\alpha}$ & Wald & LM & Wald & LM & LR \\
\hline \multirow{3}{*}{$\mathcal{N}(0,1)$} & 0 & 5.72 & 4.86 & 5.28 & 5.07 & 5.18 \\
& -0.1 & 36.34 & 35.95 & 55.06 & 54.47 & 54.77 \\
& -0.2 & 89.18 & 90.53 & 98.86 & 98.81 & 98.83 \\
\hline \multirow{3}{*}{ Laplace } & 0 & 3.34 & 4.82 & 5.27 & 5.11 & 5.19 \\
& -0.1 & 67.62 & 72.45 & 54.84 & 54.24 & 54.53 \\
& -0.2 & 99.73 & 99.80 & 98.76 & 98.70 & 98.73 \\
\hline \multirow{3}{*}{$\mathrm{t}(3)$} & 0 & 7.12 & 4.71 & 5.09 & 4.93 & 5.01 \\
& -0.1 & 67.50 & 66.93 & 55.22 & 54.64 & 54.92 \\
& -0.2 & 99.38 & 99.66 & 97.88 & 97.81 & 97.85 \\
\hline \multirow{2}{*}{$\mathrm{t}(4)$} & 0 & 5.97 & 4.74 & 5.09 & 4.90 & 5.00 \\
& -0.1 & 55.49 & 55.85 & 55.09 & 54.54 & 54.84 \\
& -0.2 & 98.28 & 98.71 & 98.58 & 98.50 & 98.55 \\
\hline \multirow{2}{*}{$\mathrm{t}(5)$} & 0 & 5.66 & 4.81 & 5.20 & 5.03 & 5.11 \\
& -0.1 & 49.67 & 50.24 & 54.89 & 54.30 & 54.61 \\
& -0.2 & 97.03 & 97.67 & 98.69 & 98.64 & 98.67 \\
\hline \multirow{2}{*}{$\mathrm{t}(10)$} & 0 & 5.74 & 4.98 & 5.22 & 5.03 & 5.13 \\
& -0.1 & 41.49 & 41.73 & 54.93 & 54.33 & 54.64 \\
& -0.2 & 93.42 & 94.33 & 98.73 & 98.69 & 98.71 \\
\hline \multirow{2}{*}{$\mathrm{t}(20)$} & 0 & 5.63 & 4.78 & 5.13 & 4.96 & 5.04 \\
& -0.1 & 38.45 & 38.50 & 55.14 & 54.50 & 54.79 \\
& -0.2 & 91.51 & 92.63 & 98.77 & 98.73 & 98.75
\end{tabular}

Table 3.9: Identical to Table 3.7, but for $T=500$. 


\begin{tabular}{|c|c|c|c|c|c|c|}
\hline & \multirow[b]{2}{*}{$\alpha$} & \multicolumn{2}{|c|}{ LAD } & \multicolumn{3}{|c|}{ OLS } \\
\hline & & Wald & $L M$ & Wald & $L M$ & $L R$ \\
\hline \multirow{3}{*}{$\varepsilon=0.01$} & 0 & 6.94 & 4.88 & 5.92 & 5.02 & 5.46 \\
\hline & -0.1 & 14.03 & 11.59 & 17.75 & 15.70 & 16.72 \\
\hline & -0.2 & 33.38 & 30.48 & 48.11 & 45.10 & 46.66 \\
\hline \multirow{3}{*}{$\varepsilon=0.02$} & 0 & 6.90 & 4.87 & 5.70 & 4.85 & 5.27 \\
\hline & -0.1 & 15.09 & 11.98 & 17.79 & 15.80 & 16.84 \\
\hline & -0.2 & 35.96 & 32.39 & 47.98 & 44.93 & 46.51 \\
\hline \multirow{3}{*}{$\varepsilon=0.03$} & 0 & 7.39 & 4.86 & 5.84 & 4.88 & 5.37 \\
\hline & -0.1 & 16.25 & 12.59 & 17.70 & 15.72 & 16.70 \\
\hline & -0.2 & 38.93 & 34.31 & 47.62 & 44.74 & 46.24 \\
\hline \multirow{3}{*}{$\varepsilon=0.04$} & 0 & 7.44 & 4.87 & 5.86 & 4.94 & 5.42 \\
\hline & -0.1 & 17.13 & 13.23 & 17.52 & 15.55 & 16.56 \\
\hline & -0.2 & 41.28 & 36.37 & 48.11 & 45.06 & 46.61 \\
\hline \multirow{3}{*}{$\varepsilon=0.05$} & 0 & 7.39 & 4.77 & 5.66 & 4.75 & 5.22 \\
\hline & -0.1 & 17.71 & 13.96 & 17.48 & 15.53 & 16.50 \\
\hline & -0.2 & 43.61 & 38.47 & 48.31 & 45.33 & 46.88 \\
\hline \multirow{3}{*}{$\varepsilon=0.10$} & 0 & 7.12 & 4.94 & 5.91 & 5.01 & 5.47 \\
\hline & -0.1 & 20.55 & 16.52 & 17.28 & 15.38 & 16.35 \\
\hline & -0.2 & 52.19 & 47.26 & 48.01 & 44.98 & 46.58 \\
\hline \multirow{3}{*}{$\varepsilon=0.15$} & 0 & 6.47 & 4.91 & 5.79 & 4.91 & 5.38 \\
\hline & -0.1 & 21.98 & 18.75 & 17.28 & 15.41 & 16.33 \\
\hline & -0.2 & 57.45 & 53.48 & 48.08 & 45.06 & 46.60 \\
\hline \multirow{3}{*}{$\varepsilon=0.20$} & 0 & 5.79 & 4.62 & 5.85 & 5.00 & 5.44 \\
\hline & -0.1 & 22.58 & 20.48 & 17.61 & 15.64 & 16.64 \\
\hline & -0.2 & 61.26 & 58.21 & 48.12 & 45.07 & 46.61 \\
\hline \multirow{3}{*}{$\varepsilon=0.25$} & 0 & 5.24 & 4.85 & 5.91 & 5.05 & 5.48 \\
\hline & -0.1 & 23.01 & 22.06 & 17.64 & 15.62 & 16.59 \\
\hline & -0.2 & 63.49 & 61.39 & 48.17 & 45.07 & 46.69 \\
\hline \multirow{3}{*}{$\varepsilon=0.50$} & 0 & 2.96 & 4.94 & 5.98 & 4.99 & 5.47 \\
\hline & -0.1 & 17.39 & 22.36 & 17.68 & 15.72 & 16.72 \\
\hline & -0.2 & 59.35 & 61.45 & 47.99 & 45.04 & 46.59 \\
\hline
\end{tabular}

Table 3.10: The empirical size and power (in percentages) of the test statistics for a varying percentage of outliers in the mixture distribution. The desired nominal size is $5 \%$. The DGP is given in Equation (3.15). The sample size is $T=100$. 


\begin{tabular}{|c|c|c|c|c|c|c|}
\hline & \multirow[b]{2}{*}{$\alpha$} & \multicolumn{2}{|c|}{ LAD } & \multicolumn{3}{|c|}{ OLS } \\
\hline & & Wald & $L M$ & Wald & $L M$ & $L R$ \\
\hline \multirow{3}{*}{$\varepsilon=0.01$} & 0 & 6.51 & 4.87 & 5.39 & 5.02 & 5.21 \\
\hline & -0.1 & 23.74 & 21.63 & 31.73 & 30.66 & 31.19 \\
\hline & -0.2 & 65.34 & 65.98 & 83.81 & 83.07 & 83.45 \\
\hline \multirow{3}{*}{$\varepsilon=0.02$} & 0 & 6.98 & 4.82 & 5.28 & 4.93 & 5.10 \\
\hline & -0.1 & 26.03 & 23.33 & 31.91 & 30.78 & 31.37 \\
\hline & -0.2 & 69.43 & 70.02 & 83.75 & 82.99 & 83.37 \\
\hline \multirow{3}{*}{$\varepsilon=0.03$} & 0 & 7.01 & 4.69 & 5.28 & 4.92 & 5.08 \\
\hline & -0.1 & 28.14 & 25.33 & 31.96 & 30.83 & 31.38 \\
\hline & -0.2 & 72.86 & 73.52 & 83.94 & 83.18 & 83.56 \\
\hline \multirow{3}{*}{$\varepsilon=0.04$} & 0 & 7.15 & 4.71 & 5.35 & 4.98 & 5.15 \\
\hline & -0.1 & 29.94 & 26.80 & 31.90 & 30.85 & 31.39 \\
\hline & -0.2 & 75.87 & 76.53 & 83.47 & 82.70 & 83.09 \\
\hline \multirow{3}{*}{$\varepsilon=0.05$} & 0 & 7.20 & 4.71 & 5.14 & 4.80 & 4.97 \\
\hline & -0.1 & 31.60 & 28.30 & 31.89 & 30.87 & 31.41 \\
\hline & -0.2 & 78.67 & 79.28 & 83.57 & 82.87 & 83.22 \\
\hline \multirow{3}{*}{$\varepsilon=0.10$} & 0 & 7.00 & 4.71 & 5.24 & 4.87 & 5.06 \\
\hline & -0.1 & 38.60 & 35.74 & 31.67 & 30.58 & 31.12 \\
\hline & -0.2 & 87.34 & 88.52 & 83.66 & 82.91 & 83.32 \\
\hline \multirow{3}{*}{$\varepsilon=0.15$} & 0 & 6.48 & 4.72 & 5.38 & 5.05 & 5.20 \\
\hline & -0.1 & 43.53 & 41.64 & 31.79 & 30.74 & 31.28 \\
\hline & -0.2 & 91.97 & 93.04 & 83.62 & 82.86 & 83.25 \\
\hline \multirow{3}{*}{$\varepsilon=0.20$} & 0 & 5.89 & 4.73 & 5.43 & 5.07 & 5.25 \\
\hline & -0.1 & 47.02 & 46.43 & 31.88 & 30.77 & 31.33 \\
\hline & -0.2 & 94.42 & 95.30 & 83.80 & 83.08 & 83.43 \\
\hline \multirow{3}{*}{$\varepsilon=0.25$} & 0 & 5.51 & 4.80 & 5.39 & 5.04 & 5.21 \\
\hline & -0.1 & 49.42 & 49.38 & 31.84 & 30.76 & 31.32 \\
\hline & -0.2 & 95.80 & 96.42 & 83.91 & 83.16 & 83.54 \\
\hline \multirow{3}{*}{$\varepsilon=0.50$} & 0 & 3.63 & 4.85 & 5.32 & 4.93 & 5.12 \\
\hline & -0.1 & 47.42 & 51.36 & 31.97 & 30.91 & 31.42 \\
\hline & -0.2 & 96.06 & 96.50 & 83.93 & 83.20 & 83.57 \\
\hline
\end{tabular}

Table 3.11: Identical to Table 3.10, but for $T=250$. 


\begin{tabular}{|c|c|c|c|c|c|c|}
\hline & \multirow[b]{2}{*}{$\alpha$} & \multicolumn{2}{|c|}{ LAD } & \multicolumn{3}{|c|}{ OLS } \\
\hline & & Wald & $L M$ & Wald & $L M$ & $L R$ \\
\hline \multirow{3}{*}{$\varepsilon=0.01$} & 0 & 6.08 & 4.80 & 5.16 & 5.00 & 5.08 \\
\hline & -0.1 & 40.79 & 39.82 & 55.36 & 54.74 & 55.06 \\
\hline & -0.2 & 92.15 & 93.28 & 98.69 & 98.65 & 98.67 \\
\hline \multirow{3}{*}{$\varepsilon=0.02$} & 0 & 6.56 & 4.83 & 5.16 & 5.00 & 5.07 \\
\hline & -0.1 & 44.41 & 43.12 & 55.12 & 54.49 & 54.81 \\
\hline & -0.2 & 94.03 & 95.11 & 98.60 & 98.55 & 98.58 \\
\hline \multirow{3}{*}{$\varepsilon=0.03$} & 0 & 6.73 & 4.87 & 5.22 & 5.05 & 5.14 \\
\hline & -0.1 & 47.51 & 46.26 & 55.10 & 54.46 & 54.77 \\
\hline & -0.2 & 95.56 & 96.46 & 98.56 & 98.50 & 98.53 \\
\hline \multirow{3}{*}{$\varepsilon=0.04$} & 0 & 6.81 & 4.69 & 5.17 & 5.01 & 5.09 \\
\hline & -0.1 & 50.56 & 49.54 & 55.15 & 54.54 & 54.86 \\
\hline & -0.2 & 96.58 & 97.48 & 98.51 & 98.46 & 98.49 \\
\hline \multirow{3}{*}{$\varepsilon=0.05$} & 0 & 6.85 & 4.79 & 5.22 & 5.06 & 5.13 \\
\hline & -0.1 & 53.66 & 52.69 & 55.39 & 54.74 & 55.07 \\
\hline & -0.2 & 97.39 & 98.19 & 98.54 & 98.48 & 98.51 \\
\hline \multirow{3}{*}{$\varepsilon=0.10$} & 0 & 6.64 & 4.81 & 5.32 & 5.14 & 5.22 \\
\hline & -0.1 & 64.59 & 64.44 & 55.00 & 54.40 & 54.70 \\
\hline & -0.2 & 99.27 & 99.61 & 98.50 & 98.46 & 98.48 \\
\hline \multirow{3}{*}{$\varepsilon=0.15$} & 0 & 6.29 & 4.76 & 5.13 & 4.97 & 5.05 \\
\hline & -0.1 & 71.96 & 72.84 & 55.10 & 54.43 & 54.79 \\
\hline & -0.2 & 99.76 & 99.90 & 98.56 & 98.52 & 98.53 \\
\hline \multirow{3}{*}{$\varepsilon=0.20$} & 0 & 6.03 & 4.80 & 5.27 & 5.10 & 5.20 \\
\hline & -0.1 & 76.98 & 77.77 & 54.96 & 54.36 & 54.65 \\
\hline & -0.2 & 99.92 & 99.97 & 98.65 & 98.60 & 98.63 \\
\hline \multirow{3}{*}{$\varepsilon=0.25$} & 0 & 5.72 & 4.81 & 5.20 & 5.00 & 5.10 \\
\hline & -0.1 & 80.06 & 81.36 & 55.09 & 54.55 & 54.80 \\
\hline & -0.2 & 99.95 & 99.98 & 98.64 & 98.59 & 98.62 \\
\hline \multirow{3}{*}{$\varepsilon=0.50$} & 0 & 4.17 & 4.84 & 5.11 & 4.95 & 5.02 \\
\hline & -0.1 & 81.11 & 82.84 & 54.95 & 54.33 & 54.64 \\
\hline & -0.2 & 99.97 & 99.98 & 98.77 & 98.73 & 98.75 \\
\hline
\end{tabular}

Table 3.12: Identical to Table 3.10, but for $T=500$. 


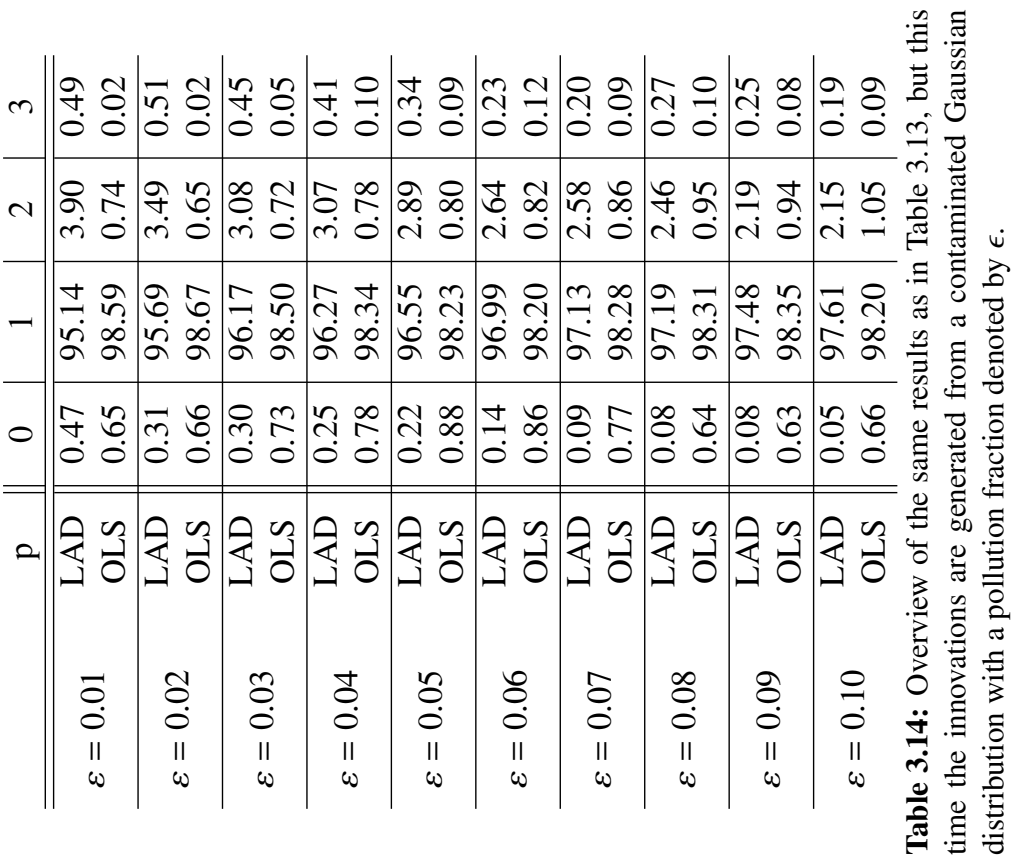

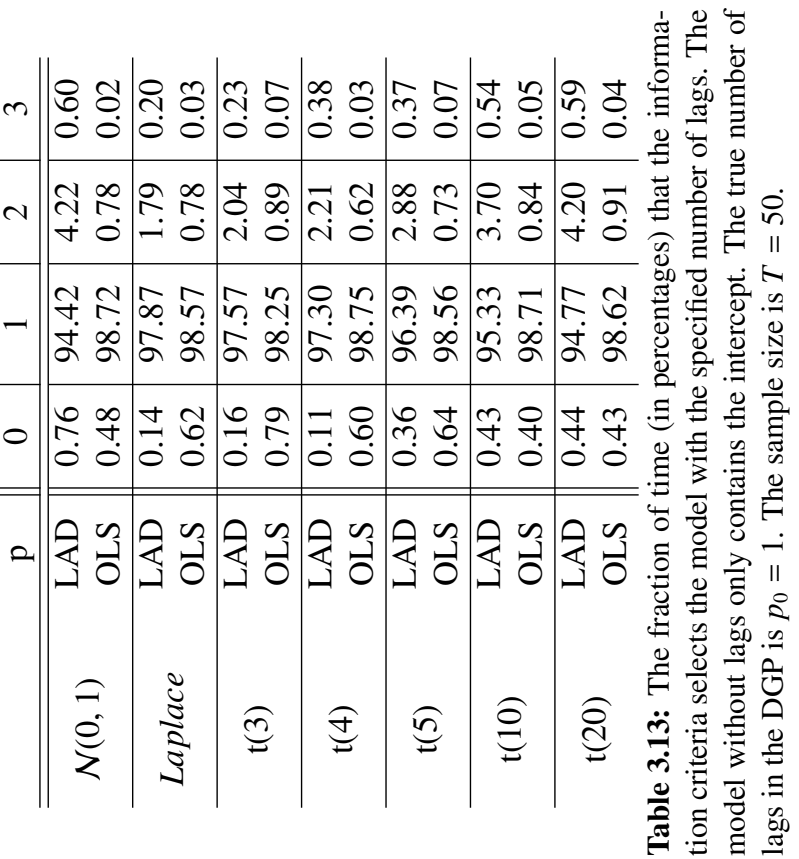




\section{A Appendix}

\section{A.1 Implementation of the Linear Program}

We follow the approach of chapter 6 in Bloomfield and Steiger (1983). To write the objective function in Equation (3.3) as a linear program (LP), we introduce a set of new variables $\eta_{k t}$. By observing that the inequality constraints below imply that $\eta_{k t} \geq \mid y_{k t}-$ $z_{t-1}^{\prime}\left(R_{k} \gamma+\boldsymbol{r}_{k}\right) \mid$, it is easy to see that our proposed estimator solves

$$
\begin{array}{ll}
\min & \sum_{k=1}^{K} \sum_{t=1}^{T} \eta_{k t} \\
\text { s.t. } & \eta_{k t} \geq y_{k t}-\boldsymbol{z}_{t-1}^{\prime}\left(\boldsymbol{R}_{k} \gamma+\boldsymbol{r}_{k}\right) \\
& \eta_{k t} \geq-y_{k t}+\boldsymbol{z}_{t-1}^{\prime}\left(\boldsymbol{R}_{k} \gamma+\boldsymbol{r}_{k}\right) .
\end{array}
$$

Now write $\boldsymbol{y}=\operatorname{vec}(Y)$ and let $\boldsymbol{\eta}$ denote the $(K T \times 1)$ vector obtained by stacking $\eta_{k t}$ across time and subsequently along the cross-sectional dimension. The LP of Equation (3.17) above can now be written in matrix form as

$$
\begin{array}{ll}
\min & {\left[\begin{array}{cc}
\boldsymbol{0}_{M}^{\prime} & \boldsymbol{\imath}_{K T}^{\prime}
\end{array}\right]\left[\begin{array}{l}
\gamma \\
\boldsymbol{\eta}
\end{array}\right]} \\
\text { s.t. } & {\left[\begin{array}{cc}
-\left(\boldsymbol{I}_{K} \otimes \boldsymbol{Z}^{\prime}\right) \boldsymbol{R} & -I_{K T} \\
\left(\boldsymbol{I}_{K} \otimes \boldsymbol{Z}^{\prime}\right) \boldsymbol{R} & -I_{K T}
\end{array}\right]\left[\begin{array}{l}
\gamma \\
\boldsymbol{\eta}
\end{array}\right] \leq\left[\begin{array}{cc}
-\boldsymbol{I}_{K T} & \left(\boldsymbol{I}_{K} \otimes \boldsymbol{Z}^{\prime}\right) \\
\boldsymbol{I}_{K T} & -\left(\boldsymbol{I}_{K} \otimes \boldsymbol{Z}^{\prime}\right)
\end{array}\right]\left[\begin{array}{l}
\boldsymbol{y} \\
\boldsymbol{r}
\end{array}\right]}
\end{array}
$$

This LP is in standard form and solvers are readily available. See Portnoy and Koenker (1997) for further computational aspects of quantile estimators (which contain LAD estimators as a special case).

\section{A.2 Proof of Theorem 1: Consistency}

Our consistency proof is based on Corollary 5.11 of White (1996). Similar methods of proof have been employed by Weiss (1991), and Kim and White (2003). We one-byone consider the requirements of Corollary 5.11. That is, we show uniform convergence, continuity of the limiting function and identifiable uniqueness. Note that Assumption 3.1 and Assumption 3.2(b) imply that

$$
\boldsymbol{y}_{t}=\boldsymbol{\mu}+\sum_{j=0}^{\infty} \boldsymbol{A}_{j} \boldsymbol{u}_{t-j}
$$


where $\boldsymbol{A}_{1}=\boldsymbol{I}_{K}$. For $r \geq 1$, we have $\mathrm{E}\left\|\boldsymbol{y}_{t}\right\|^{r} \leq\|\boldsymbol{\mu}\|^{r}+\sum_{j=0}^{\infty}\left\|\boldsymbol{A}_{j}\right\|^{r} \mathrm{E}\left\|\boldsymbol{u}_{t-j}\right\|^{r} \leq\|\boldsymbol{\mu}\|^{r}+$ $\sup _{t} \mathrm{E}\left\|\boldsymbol{u}_{t}\right\|^{r} \sum_{j=0}^{\infty}\left\|\boldsymbol{A}_{j}\right\|^{r}$. Every components of $\boldsymbol{A}_{j}$ is bounded in absolute value by a geometrically declining sequence (see Chapter 6 of Hayashi (2000)). A uniform bound on $\mathrm{E}\left\|\boldsymbol{u}_{t}\right\|^{r}$ will thus provide a uniform bound on $\mathrm{E}\left\|\boldsymbol{y}_{t}\right\|^{r}<\infty$.

\section{Uniform convergence and continuity of the limiting function}

These two properties are proven along the lines of Chapter 3 and 4 of Gallant and White (1988). The sequence $\left\{\boldsymbol{u}_{t}\right\}$ is mixing in view of Assumption 3.2(a). Define the function $q_{t}: \Omega \times \Gamma \rightarrow \mathbb{R}$ as $q_{t}(\gamma)=\sum_{k=1}^{K}\left|y_{k t}-\boldsymbol{z}_{t-1}^{\prime}\left(\boldsymbol{R}_{k} \gamma+\boldsymbol{r}_{k}\right)\right|$. This function is convex in $\gamma$ and hence Lipschitz (see e.g. Roberts and Varberg (1974) for a proof). Almost surely Lipschitz- $L_{1}$ requires a moment condition on the $L_{1}$-norm of the averaged Lipschitz functions. For $\gamma^{(1)}, \gamma^{(2)} \in \Gamma$, we have

$$
\begin{aligned}
\left|q_{t}\left(\gamma^{(1)}\right)-q_{t}\left(\boldsymbol{\gamma}^{(2)}\right)\right| & =\left|\sum_{k=1}^{K}\left(\left|y_{k t}-\boldsymbol{z}_{t-1}^{\prime}\left(\boldsymbol{R}_{k} \gamma^{(1)}+\boldsymbol{r}_{k}\right)\right|-\left|y_{k t}-\boldsymbol{z}_{t-1}^{\prime}\left(\boldsymbol{R}_{k} \gamma^{(2)}+\boldsymbol{r}_{k}\right)\right|\right)\right| \\
& \leq \sum_{k=1}^{K}|| y_{k t}-\boldsymbol{z}_{t-1}^{\prime}\left(\boldsymbol{R}_{k} \gamma^{(1)}+\boldsymbol{r}_{k}\right)|-| y_{k t}-\boldsymbol{z}_{t-1}^{\prime}\left(\boldsymbol{R}_{k} \gamma^{(2)}+\boldsymbol{r}_{k}\right)|| \\
& \leq \sum_{k=1}^{K}\left|\boldsymbol{z}_{t-1}^{\prime} \boldsymbol{R}_{k}\left(\boldsymbol{\gamma}^{(2)}-\gamma^{(1)}\right)\right| \leq\left(\sum_{k=1}^{K}\left|\boldsymbol{R}_{k}^{\prime} \boldsymbol{z}_{t-1}\right|\right)\left\|\gamma^{(2)}-\gamma^{(1)}\right\| \\
& \leq\left\|\boldsymbol{z}_{t-1}\right\|\left(\sum_{k=1}^{K}\left\|\boldsymbol{R}_{k}\right\|\right)\left\|\gamma^{(2)}-\gamma^{(1)}\right\|
\end{aligned}
$$

by the triangle inequality, the reverse triangle inequality, the Cauchy-Schwartz inequality and the submultiplicative property of the 2-norm: $\|A B\| \leq\|A\|\|B\|$. In the notation of Definition 3.5 of Gallant and White (1988) we take $a_{t}^{0}(\delta)=\delta, L_{t}^{0}=\left\|\boldsymbol{z}_{t-1}\right\|\left(\sum_{k=1}^{K}\left\|\boldsymbol{R}_{k}\right\|\right)$ and $\rho\left(\gamma^{(2)}, \gamma^{(1)}\right)=\left\|\gamma^{(2)}-\gamma^{(1)}\right\|$. The expectations of the Lipschitz functions $L_{t}^{0}$ are bounded in view of Assumption 3.2(b) and hence the sequence $\left\{q_{t}: \Omega \times \Gamma \rightarrow \mathbb{R}\right\}$ is almost surely Lipschitz- $L_{1}$. 
To derive the domination condition we use the inequality: $\left|\sum_{i=1}^{m} a_{i}\right|^{s} \leq m^{s-1} \sum_{i=1}^{m}\left|a_{i}\right|^{s}$ for $s \geq 1$. This inequality is implied by convexity. We first bound $q(\gamma)$,

$$
\begin{aligned}
\left|q_{t}(\gamma)\right| & \leq \sum_{k=1}^{K}\left|y_{k t}-\boldsymbol{z}_{t-1}^{\prime}\left(\boldsymbol{R}_{k} \gamma+\boldsymbol{r}_{k}\right)\right| \leq \sum_{k=1}^{K}\left(\left|y_{k t}\right|+\left|\boldsymbol{z}_{t-1}^{\prime}\left(\boldsymbol{R}_{k} \gamma+\boldsymbol{r}_{k}\right)\right|\right) \\
& \leq \sum_{k=1}^{K}\left|y_{k t}\right|+\left\|\boldsymbol{z}_{t-1}\right\|\|\gamma\| \sum_{k=1}^{K}\left\|\boldsymbol{R}_{k}\right\|+\left\|\boldsymbol{z}_{t-1}\right\| \sum_{k=1}^{K}\left\|\boldsymbol{r}_{k}\right\| \\
& \leq K^{1 / 2}\left\|\boldsymbol{y}_{t}\right\|+\left\|\boldsymbol{z}_{t-1}\right\|\|\gamma\| \sum_{k=1}^{K}\left\|\boldsymbol{R}_{k}\right\|+\left\|\boldsymbol{z}_{t-1}\right\| \sum_{k=1}^{K}\left\|\boldsymbol{r}_{k}\right\|,
\end{aligned}
$$

where the aforementioned inequality with $s=2$ has been used in the final step to relate the $\|\cdot\|_{1}$-norm to the $\|\cdot\|$-norm. Applying the inequality $\left|\sum_{i=1}^{m} a_{i}\right|^{s} \leq m^{s-1} \sum_{i=1}^{m}\left|a_{i}\right|^{s}$ a second time gives

$$
\mathrm{E}\left|q_{t}(\gamma)\right|^{r} \leq 3^{r-1} K^{r / 2} \mathrm{E}\left\|\boldsymbol{y}_{t}\right\|^{r}+3^{r-1} \mathrm{E}\left\|\boldsymbol{z}_{t-1}\right\|^{r}\|\gamma\|^{r}\left(\sum_{k=1}^{K}\left\|\boldsymbol{R}_{k}\right\|\right)^{r}+3^{r-1} \mathrm{E}\left\|\boldsymbol{z}_{t-1}\right\|^{r}\left(\sum_{k=1}^{K}\left\|\boldsymbol{r}_{k}\right\|\right),
$$

for any $r \geq 1$. $q_{t}(\gamma)$ is thus $r$-dominated on $\Gamma$ uniformly in $t$, if $\mathrm{E}\left\|\boldsymbol{u}_{t}\right\|^{r}$ is uniformly bounded. From Assumption 3.2 we conclude that $q_{t}(\gamma)$ is $(2+\delta)$-dominated.

To show near epoch dependence (NED) of $q_{t}$ on $\left\{\boldsymbol{u}_{t}\right\}$ of size $-1 / 2$ on $(\Gamma, \rho)$, we will apply Theorem 4.5 of Gallant and White (1988). $q_{t}$ is 2-dominated in view of Equation (3.22) and Assumption 3.2(b). We will now explicitly denote the dependence of $q_{t}$ on both the stochastic processes and the parameters and write $q_{t}\left(\boldsymbol{y}_{t}, \boldsymbol{z}_{t-1} ; \gamma\right)$. For $\hat{\boldsymbol{y}}_{m, t}$ and $\hat{z}_{m, t-1}$ to be specified later we have

$$
\begin{aligned}
& \left|q_{t}\left(\boldsymbol{y}_{t}, \boldsymbol{z}_{t-1} ; \gamma\right)-q_{t}\left(\hat{\boldsymbol{y}}_{m, t}, \hat{\boldsymbol{z}}_{m, t-1} ; \gamma\right)\right| \leq \sum_{k=1}^{K}\left|\left(y_{k t}-\hat{y}_{m, k t}\right)-\left(\boldsymbol{z}_{t-1}-\hat{\boldsymbol{z}}_{m, t-1}\right)^{\prime}\left(\boldsymbol{R}_{k} \gamma+\boldsymbol{r}_{k}\right)\right| \\
& \leq K\left\|\boldsymbol{y}_{t}-\hat{\boldsymbol{y}}_{m, t}\right\|+\left\|\boldsymbol{z}_{t-1}-\hat{\boldsymbol{z}}_{m, t-1}\right\|\left\|\sum_{k=1}^{K}\left(\boldsymbol{R}_{k} \gamma+\boldsymbol{r}_{k}\right)\right\| \\
& \quad \leq \max \left\{K,\left\|\sum_{k=1}^{K}\left(\boldsymbol{R}_{k} \gamma+\boldsymbol{r}_{k}\right)\right\|\right\}\left(\left\|\boldsymbol{y}_{t}-\hat{\boldsymbol{y}}_{m, t}\right\|+\left\|\boldsymbol{z}_{t-1}-\hat{\boldsymbol{z}}_{m, t-1}\right\|\right) \leq B(\gamma) d\left(\boldsymbol{x}_{t}, \hat{\boldsymbol{x}}_{m, t}\right)
\end{aligned}
$$


with $B(\gamma)=\max \left\{K,\left\|\sum_{k=1}^{K}\left(\boldsymbol{R}_{k} \gamma+\boldsymbol{c}_{k}\right)\right\|\right\}, d_{t}\left(\boldsymbol{x}_{t}, \hat{\boldsymbol{x}}_{m, t}\right)=\left(\left\|\boldsymbol{y}_{t}-\hat{\boldsymbol{y}}_{m, t}\right\|+\left\|\boldsymbol{z}_{t-1}-\hat{\boldsymbol{z}}_{m, t-1}\right\|\right)$ and by letting $\boldsymbol{x}_{t}=\left(\boldsymbol{y}_{t}^{\prime}, \boldsymbol{z}_{t-1}^{\prime}\right)^{\prime}$. The use of inequalities is similar to Equation (3.20). $B(\gamma)$ is bounded almost surely and hence we only have to consider the conditions Theorem 4.5 of Gallant and White (1988) places on $d\left(\boldsymbol{x}_{t}, \hat{\boldsymbol{x}}_{m, t}\right)$. We now specify $\hat{\boldsymbol{x}}_{m, t}$. Set $\mathcal{F}_{t-m}^{t+m}=$ $\sigma\left(\boldsymbol{u}_{t-m}, \ldots, \boldsymbol{u}_{t+m}\right)$ and consider the choice $\hat{\boldsymbol{x}}_{m, t}=\mathrm{E}\left(\boldsymbol{x}_{t} \mid \mathcal{F}_{t-m}^{t+m}\right)$. From $\left\|\left[\begin{array}{c}\boldsymbol{a} \\ \boldsymbol{b}\end{array}\right]\right\|^{2}=\|\boldsymbol{a}\|^{2}+\|\boldsymbol{b}\|^{2}$ we can conclude by the $c_{r}$-inequality that $\left\|\left[\begin{array}{l}\boldsymbol{a} \\ \boldsymbol{b}\end{array}\right]\right\| \leq\|\boldsymbol{a}\|+\|\boldsymbol{b}\|$. Since the lags of $\boldsymbol{y}_{t}$ are NED on $\left\{\boldsymbol{u}_{t}\right\}$ if $\boldsymbol{y}_{t}$ is NED on $\left\{\boldsymbol{u}_{t}\right\}$ we do not have to consider $\left\|\boldsymbol{z}_{t-1}-\hat{\boldsymbol{z}}_{m, t-1}\right\|$ separately. But for all $t$,

$$
\mathrm{E}\left\|\boldsymbol{y}_{t}-\hat{\boldsymbol{y}}_{m, t}\right\|=\mathrm{E}\left\|\sum_{j=m+1}^{\infty} \boldsymbol{A}_{j}\left(\boldsymbol{u}_{t-j}-\mathrm{E}\left(\boldsymbol{u}_{t-j} \mid \mathcal{F}_{t-m}^{t+m}\right)\right)\right\| \leq(K+1) \sup _{t}\left\|\boldsymbol{u}_{t}\right\| \sum_{j=m+1}^{\infty}\left\|\boldsymbol{A}_{j}\right\|,
$$

by the conditional Jensen inequality (see Result 10.18 of Davidson (1994)). ${ }^{14}$ The RHS of Equation (3.24) decays to zero exponentially fast in $m$ since the elements in the coefficient matrices are bounded by an exponentially declining series. Finally in view of footnote 14 and the $c_{r}$-inequality,

$$
\begin{aligned}
\left|d_{t}\left(\boldsymbol{x}_{t}, \hat{\boldsymbol{x}}_{m, t}\right)\right|^{2+\delta} & =\left(\left\|\boldsymbol{y}_{t}-\hat{\boldsymbol{y}}_{m, t}\right\|+\left\|\boldsymbol{z}_{t-1}-\hat{\boldsymbol{z}}_{m, t-1}\right\|\right)^{2+\delta} \leq\left(\sum_{j=1}^{p}\left\|\boldsymbol{y}_{t+1-j}-\hat{\boldsymbol{y}}_{m, t+1-j}\right\|\right)^{2+\delta} \\
& =(2 p)^{r-1}(K+1) \sum_{j=1}^{p}\left\|\boldsymbol{y}_{t+1-j}\right\|^{2+\delta}
\end{aligned}
$$

for some $\delta>0$. The expectation hereof is finite for some $\delta>2$ according to Assumption 3.2(b). Theorem 4.5 is applicable thereby showing that $\left\{q_{t}\right\}$ is near epoch dependent on $\left\{\boldsymbol{u}_{t}\right\}$ of size $-a$ for $a$ arbitrarily large on $(\Gamma, \rho)$. Together with the mixing conditions of Assumption 3.2(a), this implies that $q_{t}$ is an $L_{2}$ mixingale of size $-1 / 2$. The strong LLN by McLeish (1975) is applicable, or

$$
\frac{1}{T} \sum_{t=1}^{T} \sum_{k=1}^{K}\left[\left|y_{k t}-\boldsymbol{z}_{t-1}^{\prime}\left(\boldsymbol{R}_{k} \gamma+\boldsymbol{r}_{k}\right)\right|-\mathrm{E}\left(\left|y_{k t}-\boldsymbol{z}_{t-1}^{\prime}\left(\boldsymbol{R}_{k} \gamma+\boldsymbol{r}_{k}\right)\right|\right)\right] \rightarrow 0
$$

a.s. uniformly on $\Gamma$. Consistency follows if we can proof identifiable uniqueness.

\footnotetext{
${ }^{14}$ Note that for any $K$-vector $\boldsymbol{x}: \mathrm{E}\left\|\mathrm{E}\left(\boldsymbol{x} \mid \mathcal{F}_{t-m}^{t+m}\right)\right\| \leq \mathrm{E}\left\|\mathrm{E}\left(\boldsymbol{x} \mid \mathcal{F}_{t-m}^{t+m}\right)\right\|_{1}=\sum_{k=1}^{K} \mathrm{E}\left(\left|\mathrm{E}\left(x_{k} \mid \mathcal{F}_{t-m}^{t+m}\right)\right|\right) \leq \sum_{k=1}^{K} \mathrm{E}\left|x_{k}\right| \leq$ $\mathrm{E}\|\boldsymbol{x}\|_{1} \leq K \mathrm{E}\|\boldsymbol{x}\|$ by the ordering of vector norms. The second inequality follows from the conditional Jensen inequality.
} 


\section{Identifiable uniqueness}

To prove identifiable uniqueness we have to show

$$
\frac{1}{T} \sum_{t=1}^{T} \sum_{k=1}^{K} \mathrm{E}\left(\left|y_{k t}-z_{t-1}^{\prime}\left(\boldsymbol{R}_{k} \gamma+\boldsymbol{r}_{k}\right)\right|-\left|y_{k t}-\boldsymbol{z}_{t-1}^{\prime}\left(\boldsymbol{R}_{k} \gamma_{0}+\boldsymbol{r}_{k}\right)\right|\right) \geq 0
$$

for all $\gamma \in \Gamma$ and equality iff $\gamma=\gamma_{0}$. By Knight's identity (see page 758 of Knight (1988)) we have

$$
\begin{aligned}
& \left|y_{k t}-\boldsymbol{z}_{t-1}^{\prime}\left(\boldsymbol{R}_{k} \boldsymbol{\gamma}+\boldsymbol{r}_{k}\right)\right|-\left|y_{k t}-\boldsymbol{z}_{t-1}^{\prime}\left(\boldsymbol{R}_{k} \gamma_{0}+\boldsymbol{r}_{k}\right)\right|=\left|u_{k t}-\boldsymbol{z}_{t-1}^{\prime} \boldsymbol{R}_{k}\left(\gamma-\gamma_{0}\right)\right|-\left|u_{k t}\right| \\
& =-\boldsymbol{z}_{t-1}^{\prime} \boldsymbol{R}_{k}\left(\boldsymbol{\gamma}-\gamma_{0}\right)\left[\mathbb{1}_{\left\{u_{k t}>0\right\}}-\mathbb{1}_{\left\{u_{k t}<0\right\}}\right]+2 \int_{0}^{\boldsymbol{z}_{t-1}^{\prime} \boldsymbol{R}_{k}\left(\boldsymbol{\gamma}-\boldsymbol{\gamma}_{0}\right)}\left(\mathbb{1}_{\left\{u_{k t} \leq s\right\}}-\mathbb{1}_{\left\{u_{k t} \leq 0\right\}}\right) d s .
\end{aligned}
$$

Now define $\delta_{k t}=\boldsymbol{z}_{t-1}^{\prime} \boldsymbol{R}_{k}\left(\gamma-\gamma_{0}\right)$, and use indicators to distinguish between $\left\{\delta_{k t} \geq 0\right\}$ and $\left\{\delta_{k t}<0\right\}$,

$$
\begin{aligned}
\mathrm{E}_{t-1} & \int_{0}^{\delta_{k t}}\left(\mathbb{1}_{\left\{u_{k t} \leq s\right\}}-\mathbb{1}_{\left\{u_{k t} \leq 0\right\}}\right) d s \\
& =\mathbb{1}_{\left\{\delta_{k t} \geq 0\right\}} \int_{0}^{\delta_{k t}} \int_{0}^{s} f_{k, t}(x) d x d s+\mathbb{1}_{\left\{\delta_{k t}<0\right\}} \int_{-\left|\delta_{k t}\right|}^{0} \int_{s}^{0} f_{k, t}(x) d x d s \\
& =\mathbb{1}_{\left\{\delta_{k t} \geq 0\right\}} \int_{0}^{\delta_{k t}}\left(\delta_{k t}-x\right) f_{k, t}(x) d x+\mathbb{1}_{\left\{\delta_{k t}<0\right\}} \int_{-\left|\delta_{k t}\right|}^{0}\left(x+\left|\delta_{k t}\right|\right) f_{k, t}(x) d x \\
& \geq \mathbb{1}_{\left\{\delta_{k t} \geq 0\right\}} \int_{0}^{\delta_{k t}}\left(\delta_{k t}-x\right) f_{k}^{*} d x+\mathbb{1}_{\left\{\delta_{k t}<0\right\}} \int_{-\left|\delta_{k t}\right|}^{0}\left(x+\left|\delta_{k t}\right|\right) f_{k}^{*} d x=\frac{1}{2} \delta_{k t}^{2} f_{k}^{*},
\end{aligned}
$$

where the second to third line is a change in the order of integration and the lower bound $f_{k}^{*}=\inf _{t} f_{k, t}(x)$ has been used. The conditional median zero assumption on the error terms makes sure that the first term in Equation (3.28) vanishes under $\mathrm{E}_{t-1}$. Overall we have

$$
\begin{aligned}
& \frac{1}{T} \sum_{t=1}^{T} \sum_{k=1}^{K} \mathrm{E}\left(\left|y_{k t}-\boldsymbol{z}_{t-1}^{\prime}\left(\boldsymbol{R}_{k} \gamma+\boldsymbol{r}_{k}\right)\right|-\left|y_{k t}-\boldsymbol{z}_{t-1}^{\prime}\left(\boldsymbol{R}_{k} \gamma_{0}+\boldsymbol{r}_{k}\right)\right|\right) \\
& =\frac{2}{T} \sum_{t=1}^{T} \mathrm{E}\left(\sum_{k=1}^{K} \frac{1}{2} \delta_{k}^{2} f_{k}^{*}\right) \\
& \geq\left(\min _{k=1,2, \ldots, K} f_{k}^{*}\right)\left(\gamma-\gamma_{0}\right)^{\prime} \sum_{k=1}^{K} \boldsymbol{R}_{k}^{\prime}\left(\frac{1}{T} \sum_{t=1}^{T} \mathrm{E}\left(\boldsymbol{z}_{t-1} \boldsymbol{z}_{t-1}^{\prime}\right)\right) \boldsymbol{R}_{k}\left(\gamma-\gamma_{0}\right) .
\end{aligned}
$$


Assumption 3.3 ensures that the quadratic form in the RHS is nonnegative, and zero iff $\gamma=\gamma_{0}$ as $T \rightarrow \infty$.

\section{A.3 Proof of Theorem 2: Asymptotic Normality}

Outline of the proof: The proof of asymptotic normality dates back to Huber (1967). This method of proof is common if the objective function is not differentiable. Similar arguments have also been employed by Powell (1984), Weiss (1991), Kim and White (2003) and White et al. (2015) among others. We will first provide a sketch of the proof. The detailed assumptions and conditions are provided later. Define $\boldsymbol{\eta}_{t}(\gamma)=$ $-\sum_{k=1}^{K} \boldsymbol{R}_{k}^{\prime} \boldsymbol{z}_{t-1} \operatorname{sgn}\left(y_{k t}-\boldsymbol{z}_{t-1}^{\prime}\left(\boldsymbol{R}_{k} \gamma+\boldsymbol{r}_{k}\right)\right)$, which is also the gradient of the expression $\sum_{k=1}^{K}\left|y_{k t}-\boldsymbol{z}_{t-1}^{\prime}\left(\boldsymbol{R}_{k} \gamma+\boldsymbol{r}_{k}\right)\right|$ for $y_{k t}-\boldsymbol{z}_{t-1}^{\prime}\left(\boldsymbol{R}_{k} \gamma+\boldsymbol{r}_{k}\right) \neq 0$. The first requirement for the result of Huber (1967) is

$$
\frac{1}{\sqrt{T}} \sum_{t=1}^{T} \boldsymbol{\eta}_{t}\left(\widehat{\gamma}_{T}\right) \stackrel{p}{\longrightarrow} 0
$$

This expression can be interpreted as an asymptotic equivalent of the vanishing score which is encountered for differentiable objective functions. For differentiable objective functions we would subsequently use a Taylor approximation of the score around the true parameter vector. The score evaluated at the true parameter vector is then shown to satisfy a CLT and a multiplication with the inverse Hessian would provide the asymptotic normality result. This approach cannot be used in our setup due to non-differentiability of the absolute value function.

Huber (1967) circumvented this non-differentiability problem by first taking expectations. Consider the expectation $\boldsymbol{\lambda}_{T}(\gamma)=\frac{1}{T} \sum_{t=1}^{T} \mathrm{E} \boldsymbol{\eta}_{t}(\gamma)$. Sufficient regularity conditions imply that

$$
\frac{1}{\sqrt{T}} \sum_{t=1}^{T} \boldsymbol{\eta}_{t}\left(\gamma_{0}\right)+\sqrt{T} \boldsymbol{\lambda}_{T}\left(\widehat{\gamma}_{T}\right)=o_{p}(1) .
$$

The first term in Equation (3.32) will satisfies a Central Limit Theorem. The second term is the expectation of the objective function at $\widehat{\gamma}_{T}$. This expectation is thus a smoothed version of the objective function and can be differentiated. A mean value expansion, $\sqrt{T} \boldsymbol{\lambda}_{T}\left(\widehat{\gamma}_{T}\right)=\overline{\boldsymbol{H}}_{T} \sqrt{T}\left(\widehat{\gamma}_{T}-\gamma_{0}\right)+o_{p}(1)$, delivers the asymptotic distribution. The details are given now. 


\section{Proof of Equation (3.31)}

The proof originates from Ruppert and Carroll (1980). Let $\left\{\boldsymbol{e}_{s}\right\}_{s=1}^{K}$ denote the standard basis of $\mathbb{R}^{K}$, that is $\boldsymbol{e}_{s}$ is the $s^{\prime}$ th column of $\boldsymbol{I}_{K}$. We define the function $G_{j}(a)=$ $\frac{1}{\sqrt{T}} \sum_{t=1}^{T} \sum_{k=1}^{K}\left|y_{k t}-\boldsymbol{z}_{t-1}^{\prime}\left(\boldsymbol{R}_{k}\left(\widehat{\gamma}_{T}+a \boldsymbol{e}_{s}\right)+\boldsymbol{r}_{k}\right)\right|$ for any real number $a$. This is a rescaled version of the objective function and $G_{j}(a)$ will therefore attain its minimum at $a=0$ by definition of $\widehat{\gamma}_{T}$. The right derivative of $G_{j}(a)$ with respect to $a$ is

$$
H_{s}(a)=-\frac{1}{\sqrt{T}} \sum_{t=1}^{T} \sum_{k=1}^{K} \boldsymbol{e}_{s}^{\prime} \boldsymbol{R}_{k}^{\prime} \boldsymbol{z}_{t-1} \operatorname{sgn}\left(y_{k t}-\boldsymbol{z}_{t-1}^{\prime}\left(\boldsymbol{R}_{k}\left(\widehat{\gamma}_{T}+a \boldsymbol{e}_{s}\right)+\boldsymbol{r}_{k}\right)\right) .
$$

$H_{s}(a)$ is: (1) non-decreasing in $a$, and (2) satisfies $H_{s}(-\epsilon) \leq 0$ and $H_{s}(\epsilon) \geq 0$ for any $\epsilon>0$. These two statements imply that

$$
\begin{aligned}
H_{s}(0) & \leq H_{s}(\epsilon)-H_{s}(-\epsilon) \\
& =-\frac{2}{\sqrt{T}} \sum_{t=1}^{T} \sum_{k=1}^{K} \boldsymbol{e}_{s}^{\prime} \boldsymbol{R}_{k}^{\prime} \boldsymbol{z}_{t-1}\left[\mathbb{1}_{\left.\left\{y_{k t}-\boldsymbol{z}_{t-1}^{\prime}\left[\boldsymbol{R}_{k} \widehat{\gamma}_{T}-\epsilon \boldsymbol{e}_{s}\right)+\boldsymbol{r}_{k}\right]\right\}}-\mathbb{1}_{\left\{y_{k t}-\boldsymbol{z}_{t-1}^{\prime}\left[\boldsymbol{R}_{k}\left(\widehat{\gamma}_{T}+\epsilon \boldsymbol{e}_{s}\right)+\boldsymbol{r}_{k}\right]\right\}}\right]
\end{aligned}
$$

and by taking the limit $\epsilon \downarrow 0$ we obtain

$$
\begin{aligned}
\left|H_{S}(0)\right| & \leq \frac{2}{\sqrt{T}} \sum_{t=1}^{T} \sum_{k=1}^{K}\left|\boldsymbol{e}_{s}^{\prime} \boldsymbol{R}_{k}^{\prime} \boldsymbol{z}_{t-1}\right| \mathbb{1}_{\left\{y_{k t}-\boldsymbol{z}_{t-1}^{\prime}\left(\boldsymbol{R}_{k} \widehat{\gamma}_{T}+\boldsymbol{r}_{k}\right)=0\right\}} \\
& \leq \frac{2\|\boldsymbol{R}\|}{\sqrt{T}} \sup _{t}\left\|\boldsymbol{z}_{t-1}\right\| \sum_{t=1}^{T} \sum_{k=1}^{K} \mathbb{1}_{\left\{y_{k t}-\boldsymbol{z}_{t-1}^{\prime}\left(\boldsymbol{R}_{k} \widehat{\gamma}_{T}+\boldsymbol{r}_{k}\right)=0\right\}} .
\end{aligned}
$$

The supremum over $\boldsymbol{z}_{t-1}$ is $O_{p}(1)$, because $\mathrm{E}\left\|\boldsymbol{y}_{t}\right\|$ is uniformly bounded in view of Assumption 3.2(b). The number of residuals that will equal precisely zero is also $O_{p}(1)$ in view of Theorem 3.3 of Koenker and Bassett (1978) and Assumption 3.3. We conclude that $\left|H_{s}(0)\right|=o_{p}(1)$. But $H_{s}(0)$ is the $s$ 'th element of $\frac{1}{\sqrt{T}} \sum_{t=1}^{T} \boldsymbol{\eta}_{t}\left(\widehat{\gamma}_{T}\right)$ and our choice of $s$ was arbitrary. The proof of Equation (3.31) is thus complete.

\section{Proof of Equation (3.32)}

We need to verify the conditions of Section 3.B.1. $\boldsymbol{\eta}_{t}(\gamma)$ consists of sums of products of measurable functions. Measurability of $\boldsymbol{\eta}_{t}(\gamma)$ is thus garanteed. Separability follows from the compactness assumption, Assumption 3.4. Requirement $(\mathrm{N}-1)$ is fulfilled. The 
law of iterated expectations and Assumption 3.3(a) combine to

$$
\begin{aligned}
\boldsymbol{\lambda}_{T}\left(\gamma_{0}\right) & =-\mathrm{E} \frac{1}{T} \sum_{t=1}^{T} \sum_{k=1}^{K} \boldsymbol{R}_{k}^{\prime} \boldsymbol{z}_{t-1} \mathrm{E}\left(\operatorname{sgn}\left(y_{k t}-\boldsymbol{z}_{t-1}^{\prime}\left(\boldsymbol{R}_{k} \gamma_{0}+\boldsymbol{r}_{k}\right)\right) \mid \mathcal{F}_{t-1}\right) \\
& =-\mathrm{E} \frac{1}{T} \sum_{t=1}^{T} \sum_{k=1}^{K} \boldsymbol{R}_{k}^{\prime} \boldsymbol{z}_{t-1} \mathrm{E}\left(\operatorname{sgn}\left(u_{k t}\right) \mid \mathcal{F}_{t-1}\right)=\mathbf{0},
\end{aligned}
$$

and thereby establish (N-2). For (N-3)(i) we will make use of the result $\boldsymbol{\lambda}_{T}(\gamma)=\boldsymbol{\lambda}_{T}\left(\gamma_{0}\right)+$ $\overline{\mathcal{H}}_{T}\left(\gamma-\gamma_{0}\right)+O\left(\left\|\gamma-\gamma_{0}\right\|^{2}\right)$. The proof of this statement is given in the discussion culminating in Equation (3.49). By this result, there exist $d_{1}>0$ and $C>0$, such that $\left\|\boldsymbol{\lambda}_{T}(\gamma)-\overline{\mathcal{H}}_{T}\left(\gamma-\gamma_{0}\right)\right\| \leq C\left\|\gamma-\gamma_{0}\right\|^{2}$, holds over the neighborhood $\left\|\gamma-\gamma_{0}\right\| \leq d_{1}$. Assumption 3.7(b) implies that $\inf _{T \geq T_{0}} \lambda_{\text {min }}\left(\overline{\mathcal{H}}_{T}\right)>0$. For $T \geq T_{0}$ and $\left\|\gamma-\gamma_{0}\right\|<d$ with $d=\min \left\{d_{1}, \frac{1}{2} \lambda_{\min }\left(\overline{\mathcal{H}}_{T}\right) / C\right\}$, we have

$$
\begin{aligned}
\left\|\boldsymbol{\lambda}_{T}(\gamma)\right\| & =\left\|\overline{\mathcal{H}}_{T}\left(\gamma-\gamma_{0}\right)+\lambda_{T}(\gamma)-\overline{\mathcal{H}}_{T}\left(\gamma-\gamma_{0}\right)\right\| \\
& \geq\left\|\overline{\mathcal{H}}_{T}\left(\gamma-\gamma_{0}\right)\right\|-\left\|\lambda_{T}(\gamma)-\overline{\mathcal{H}}_{T}\left(\gamma-\gamma_{0}\right)\right\| \geq\left\|\overline{\mathcal{H}}_{T}\left(\gamma-\gamma_{0}\right)\right\|-C\left\|\gamma-\gamma_{0}\right\|^{2} \\
& \geq\left(\lambda_{\min }\left(\overline{\mathcal{H}}_{T}\right)-C\left\|\gamma-\gamma_{0}\right\|\right)\left\|\gamma-\gamma_{0}\right\| \geq \frac{1}{2} \lambda_{\min }\left(\overline{\mathcal{H}}_{T}\right)\left\|\gamma-\gamma_{0}\right\|,
\end{aligned}
$$

by the properties of the smallest eigenvalue and the choice of $d$. Condition (N-3)(i) is thus fulfilled. The proofs of (N-3)(ii) and (N-3)(iii) use the inequality

$$
|\operatorname{sgn}(a)-\operatorname{sgn}(a-b)|=2\left|\mathbb{1}_{\{a<b\}}-\mathbb{1}_{\{a<0\}}\right| \leq 2 \mathbb{1}_{\{|a|<|b|\}} .
$$

For any $t$ and fixed $\gamma$, a bound on $\zeta_{t}(\gamma, d)$ is

$$
\begin{aligned}
\zeta_{t}(\boldsymbol{\gamma}, d) & =\sup _{\|\boldsymbol{\tau}-\boldsymbol{\gamma}\|<d}\left\|\sum_{k=1}^{K} \boldsymbol{R}_{k}^{\prime} \boldsymbol{z}_{t-1}\left[\operatorname{sgn}\left(y_{k t}-\boldsymbol{z}_{t-1}\left(\boldsymbol{R}_{k} \boldsymbol{\gamma}+\boldsymbol{r}_{k}\right)\right)-\operatorname{sgn}\left(y_{k t}-\boldsymbol{z}_{t-1}\left(\boldsymbol{R}_{k} \boldsymbol{\tau}+\boldsymbol{r}_{k}\right)\right)\right]\right\| \\
& \leq \sum_{k=1}^{K}\left\|\boldsymbol{R}_{k} \boldsymbol{z}_{t-1}\right\| \sup _{\|\boldsymbol{\tau}-\boldsymbol{\gamma}\|<d}\left|\operatorname{sgn}\left(u_{k t}-\boldsymbol{z}_{t-1}^{\prime} \boldsymbol{R}_{k}\left(\boldsymbol{\gamma}-\gamma_{0}\right)\right)-\operatorname{sgn}\left(u_{k t}-\boldsymbol{z}_{t-1}^{\prime} \boldsymbol{R}_{k}\left(\boldsymbol{\tau}-\gamma_{0}\right)\right)\right| \\
& \leq 2\|\boldsymbol{R}\|\left\|\boldsymbol{z}_{t-1}\right\| \sum_{k=1}^{K} \sup _{\|\boldsymbol{\tau}-\boldsymbol{\gamma}\|<d} \mathbb{1}_{\left\{\left|u_{k t}-\boldsymbol{z}_{t-1}^{\prime} \boldsymbol{R}_{k}\left(\boldsymbol{\gamma}-\boldsymbol{\gamma}_{0}\right)\right|<\left|\boldsymbol{z}_{t-1}^{\prime} \boldsymbol{R}_{k}(\boldsymbol{\tau}-\boldsymbol{\gamma})\right|\right\}} \\
& \leq 2\|\boldsymbol{R}\|\left\|\boldsymbol{z}_{t-1}\right\| \sum_{k=1}^{K} \mathbb{1}_{\left\{\left|u_{k t}-\boldsymbol{z}_{t-1}^{\prime} \boldsymbol{R}_{k}\left(\boldsymbol{\gamma}-\boldsymbol{\gamma}_{0}\right)\right|<\left\|\boldsymbol{z}_{t-1}^{\prime} \boldsymbol{R}\right\| d\right\}}
\end{aligned}
$$


where the consecutive inequalities follow from the triangle inequality, the fact that $\left\|\boldsymbol{R}_{k}\right\|<$ $\|\boldsymbol{R}\|$, and the inequality given in Equation (3.38). Hölder's equality and Assumption 3.6(a) result in

$\mathrm{E} \zeta_{t}(\boldsymbol{\gamma}, d) \leq 2\|\boldsymbol{R}\| \mathrm{E}\left(\left\|\boldsymbol{z}_{t-1}\right\| \sum_{k=1}^{K} \int_{\boldsymbol{z}_{t-1}^{\prime} \boldsymbol{R}_{k}\left(\boldsymbol{\gamma}-\boldsymbol{\gamma}_{0}\right)-\left\|\boldsymbol{z}_{t-1}^{\prime} \boldsymbol{R}\right\| d}^{\boldsymbol{z}_{t-1}^{\prime} \boldsymbol{R}_{k}\left(\boldsymbol{\gamma}-\boldsymbol{\gamma}_{0}\right)+\left\|\boldsymbol{z}_{t-1}^{\prime} \boldsymbol{R}\right\| d} f_{k, t}(x) d x\right) \leq 4 f_{0} K\|\boldsymbol{R}\|^{2}\left(\mathrm{E}\left\|\boldsymbol{z}_{t-1}\right\|^{2}\right) d$,

which shows that (N-3)(i) is satisfied with $b=4 f_{0} K\|R\|^{2}\left(\mathrm{E}\left\|z_{t-1}\right\|^{2}\right)$ and $d_{0}=2 d$. The proof of (N-3)(iii) follows from Hölder's inequality, the $c_{r}$-inequality, and the same steps as in Equation (3.40),

$$
\mathrm{E} \zeta_{t}^{2}(\boldsymbol{\gamma}, d) \leq 4\|\boldsymbol{R}\|^{2} \mathrm{E}\left(\sum_{k=1}^{K}\left\|\boldsymbol{z}_{t-1}\right\| \mathbb{1}_{\left\{\left|u_{k t}-\boldsymbol{z}_{t-1}^{\prime} \boldsymbol{R}_{k}\left(\boldsymbol{\gamma}-\boldsymbol{\gamma}_{0}\right)\right|<\left\|\boldsymbol{z}_{t-1}^{\prime} \boldsymbol{R}\right\| d\right\}}\right)^{2} \leq 8 f_{0} K^{2}\|\boldsymbol{R}\|^{3}\left(\mathrm{E}\left\|\boldsymbol{z}_{t-1}\right\|^{3}\right) d,
$$

implying that we can take $c=8 f_{0} K^{2}\|\boldsymbol{R}\|^{3}\left(\mathrm{E}\left\|z_{t-1}\right\|^{3}\right)$ and $d_{0}=2 d$ as before .

\section{Derivation of the Central Limit Theorem}

We now proof that $\frac{1}{\sqrt{T}} \sum_{t=1}^{T} \boldsymbol{\eta}_{t}\left(\gamma_{0}\right)$ satisfies a central limit theorem. For any conformable vector $\boldsymbol{\theta}, X_{t}=\boldsymbol{\theta}^{\prime} \boldsymbol{\eta}_{t}\left(\gamma_{0}\right)=\boldsymbol{\theta}^{\prime} \sum_{k=1}^{K} \boldsymbol{R}_{k}^{\prime} z_{t-1} \operatorname{sgn}\left(u_{k t}\right)$ is a martingale difference sequence with respect to $\mathcal{F}_{t-1}$ because

1. $\mathrm{E}\left|\boldsymbol{\theta}^{\prime} \sum_{k=1}^{K} \boldsymbol{R}_{k}^{\prime} \boldsymbol{z}_{t-1} \operatorname{sgn}\left(u_{k t}\right)\right| \leq\|\boldsymbol{\theta}\| \sum_{k=1}^{K} \mathrm{E}\left\|\boldsymbol{R}_{k}^{\prime} \boldsymbol{z}_{t-1}\right\| \leq\|\boldsymbol{\theta}\| \mathrm{E}\left(\left\|\boldsymbol{z}_{t-1}\right\|\right) \sum_{k=1}^{K}\left\|\boldsymbol{R}_{k}\right\|<\infty$,

2. $\mathrm{E}\left(\boldsymbol{\theta}^{\prime} \sum_{k=1}^{K} \boldsymbol{R}_{k}^{\prime} z_{t-1} \operatorname{sgn}\left(u_{k t}\right) \mid \mathcal{F}_{t-1}\right)=\boldsymbol{\theta}^{\prime} \sum_{k=1}^{K} \boldsymbol{R}_{k}^{\prime} \boldsymbol{z}_{t-1} \mathrm{E}\left(\operatorname{sgn}\left(u_{k t}\right) \mid \mathcal{F}_{t-1}\right)=0$.

$\frac{1}{\sqrt{T}} \sum_{t=1}^{T} \boldsymbol{\eta}_{t}\left(\gamma_{0}\right)$ is thus a rescaled sum of a vector martingale difference sequence. By the definition of $\boldsymbol{\Psi}_{t}$ in Assumption 3.7, the conditional second moment of $X_{t}$ is $\sigma_{t}^{2}=$ $\mathrm{E}\left(X_{t}^{2} \mid \mathcal{F}_{t-1}\right)=\sum_{k_{1}=1}^{K} \sum_{k_{2}=1}^{K} \Psi_{t, k_{1} k_{2}} \boldsymbol{\theta}^{\prime} \boldsymbol{R}_{k_{1}}^{\prime} \boldsymbol{z}_{t-1} \boldsymbol{z}_{t-1}^{\prime} \boldsymbol{R}_{k_{2}} \boldsymbol{\theta}=\boldsymbol{\theta}^{\prime} \boldsymbol{R}^{\prime}\left(\boldsymbol{\Psi}_{t} \otimes \boldsymbol{z}_{t-1} \boldsymbol{z}_{t-1}^{\prime}\right) \boldsymbol{R} \boldsymbol{\theta}$. Taking expectations, the sum of the variances equals $s_{T}^{2}=\boldsymbol{\theta}^{\prime} \boldsymbol{R}^{\prime} \sum_{t=1}^{T} \mathrm{E}\left(\boldsymbol{\Psi}_{t} \otimes z_{t-1} \boldsymbol{z}_{t-1}^{\prime}\right) \boldsymbol{R} \boldsymbol{\theta}$. We will invoke Theorem 24.4 of Davidson (1994) with $c_{t}=1$. The uniform integrability 
conditions follows from the $c_{r}$-inequality and Assumption 3.2(b), that is

$$
\begin{aligned}
\mathrm{E}\left|X_{t}\right|^{2+\delta} & \leq\|\boldsymbol{\theta}\|^{2+\delta} \mathrm{E}\left\|\sum_{k=1}^{K} \boldsymbol{R}_{k}^{\prime} \boldsymbol{z}_{t-1} \operatorname{sgn}\left(u_{k t}\right)\right\|^{2+\delta} \leq\|\boldsymbol{\theta}\|^{2+\delta} K^{1+\delta} \sum_{k=1}^{K} \mathrm{E}\left\|\boldsymbol{R}_{k}^{\prime} z_{t-1} \operatorname{sgn}\left(u_{k t}\right)\right\|^{2+\delta} \\
& \leq\|\boldsymbol{\theta}\|^{2+\delta} K^{1+\delta} \mathrm{E}\left\|z_{t-1}\right\|^{2+\delta} \sum_{k=1}^{K}\left\|\boldsymbol{R}_{k}\right\|^{2+\delta}<\infty .
\end{aligned}
$$

The condition $\sup _{t} T / s_{T}^{2}<\infty$ is ensured by Assumption 3.7(a). Theorem 24.4 is thus applicable and the Cramer-Wold theorem establishes

$\left(\boldsymbol{R}^{\prime} \overline{\boldsymbol{V}}_{T} \boldsymbol{R}\right)^{-1 / 2} \frac{1}{\sqrt{T}} \sum_{t=1}^{T} \boldsymbol{\eta}_{t}\left(\gamma_{0}\right)=\left(\boldsymbol{R}^{\prime} \overline{\boldsymbol{V}}_{T} \boldsymbol{R}\right)^{-1 / 2} \frac{1}{\sqrt{T}} \sum_{t=1}^{T} \sum_{k=1}^{K} \boldsymbol{R}_{k}^{\prime} \boldsymbol{z}_{t-1} \operatorname{sgn}\left(u_{k t}\right) \stackrel{d}{\longrightarrow} \mathrm{N}\left(\mathbf{0}, \boldsymbol{I}_{M}\right)$,

with $\overline{\boldsymbol{V}}_{T}$ as defined in Assumption 3.7 .

\section{Derivation of the Mean Value Expansion}

The $(M \times 1)$ vector $\boldsymbol{\lambda}_{T}(\gamma)$ satisfies

$$
\begin{aligned}
\boldsymbol{\lambda}_{T}(\gamma) & =-\frac{1}{T} \sum_{t=1}^{T} \sum_{k=1}^{K} \mathrm{E}\left(\boldsymbol{R}_{k}^{\prime} \boldsymbol{z}_{t-1}\left(1-2 \mathbb{1}_{\left\{u_{k t}-\boldsymbol{z}_{t-1}^{\prime} \boldsymbol{R}_{k}\left(\boldsymbol{\gamma}-\boldsymbol{\gamma}_{0}\right)<0\right\}}\right)\right) \\
& =-\frac{1}{T} \sum_{t=1}^{T} \sum_{k=1}^{K} \mathrm{E}\left[\boldsymbol{R}_{k}^{\prime} \boldsymbol{z}_{t-1}\left(1-2 \int_{-\infty}^{\boldsymbol{z}_{t-1}^{\prime} \boldsymbol{R}_{k}\left(\boldsymbol{\gamma}-\boldsymbol{\gamma}_{0}\right)} f_{k, t}(x) d x\right)\right],
\end{aligned}
$$

where the second equality follows from the law of iterated expectations. Now by the chain rule,

$$
\frac{\partial}{\partial \boldsymbol{\gamma}^{\prime}}\left[\boldsymbol{R}_{k}^{\prime} \boldsymbol{z}_{t-1}\left(1-2 \int_{-\infty}^{\boldsymbol{z}_{t-1}^{\prime} \boldsymbol{R}_{k}\left(\boldsymbol{\gamma}-\boldsymbol{\gamma}_{0}\right)} f_{k, t}(x) d x\right)\right]=-2 f_{k, t}\left(\boldsymbol{z}_{t-1}^{\prime} \boldsymbol{R}_{k}\left(\boldsymbol{\gamma}-\gamma_{0}\right)\right) \boldsymbol{R}_{k}^{\prime} \boldsymbol{z}_{t-1} \boldsymbol{z}_{t-1}^{\prime} \boldsymbol{R}_{k}
$$

and $\left\|\frac{2}{T} \sum_{t=1}^{T} \sum_{k=1}^{K} \mathrm{E}\left(f_{k, t}\left(\boldsymbol{z}_{t-1}^{\prime} \boldsymbol{R}_{k}\left(\gamma-\gamma_{0}\right)\right) \boldsymbol{R}_{k}^{\prime} \boldsymbol{z}_{t-1} \boldsymbol{z}_{t-1}^{\prime} \boldsymbol{R}_{k}\right)\right\| \leq 2 f_{0}\|\boldsymbol{R}\|^{2} K \sup _{t} \mathrm{E}\left\|\boldsymbol{z}_{t-1}\right\|^{2}<$ $\infty$. Corollary 5.8 and 5.9 of Bartle (1966) imply that $\lambda_{T}(\gamma)$ is continuously differentiable on $\Gamma$. By Assumption $3.5\left(\gamma_{0} \in \operatorname{int}(\Gamma)\right)$, the mean value theorem guarantees the existence 
of a neighborhood of $\gamma_{0}$ such that

$$
\lambda_{T}(\gamma)=\lambda_{T}\left(\gamma_{0}\right)+\mathcal{H}_{T}^{*}\left(\gamma-\gamma_{0}\right)
$$

The rows of the $(M \times M)$ matrix $\mathcal{H}_{T}^{*}$ in Equation (3.46) are given by $\frac{\partial \boldsymbol{\lambda}_{T, j}\left(\boldsymbol{\gamma}_{(m)}\right)}{\partial \boldsymbol{\gamma}^{\prime}}$, where the $\gamma_{(m)}(m=1, \ldots, M)$ are located on the line segment connecting $\gamma$ and $\gamma_{0}$. The last remark also means that $\left\|\gamma_{(m)}-\gamma_{0}\right\| \leq\left\|\gamma-\gamma_{0}\right\|$ for all $m$, and this fact will be used to show that $\left\|\mathcal{H}_{T}^{*}-\overline{\mathcal{H}}_{T}\right\|=O\left(\left\|\gamma-\gamma_{0}\right\|\right)$, where

$$
\overline{\mathcal{H}}_{T}=\frac{2}{T} \sum_{t=1}^{T} \sum_{k=1}^{K} \mathrm{E}\left(f_{k, t}(0) \boldsymbol{R}_{k}^{\prime} z_{t-1} \boldsymbol{z}_{t-1}^{\prime} \boldsymbol{R}_{k}\right)=\boldsymbol{R}^{\prime}\left(\frac{2}{T} \sum_{t=1}^{T} \mathrm{E}\left(\Xi_{t} \otimes \boldsymbol{z}_{t-1} \boldsymbol{z}_{t-1}^{\prime}\right)\right) \boldsymbol{R}=\boldsymbol{R}^{\prime} \overline{\boldsymbol{H}}_{T} \boldsymbol{R} .
$$

The varying $\gamma_{(m)}$ 's require some more notation. For an $(M \times M)$ matrix $\boldsymbol{A}$, let us denote the $m$ 'th row as $\boldsymbol{A}_{m}$. The $c_{r}$-inequality gives $\|\boldsymbol{A}\| \leq \sum_{m=1}^{M}\left\|\boldsymbol{A}_{m}\right\|$. Applying the same notation to denote the rows of $\boldsymbol{R}_{k}^{\prime}$ gives

$$
\begin{aligned}
\left\|\mathcal{H}_{T}^{*}-\overline{\mathcal{H}}_{T}\right\| & \leq \sum_{m=1}^{M}\left\|\frac{2}{T} \sum_{t=1}^{T} \sum_{k=1}^{K} \mathrm{E}\left(\left[f_{k, t-1}\left(\boldsymbol{z}_{t-1}^{\prime} \boldsymbol{R}_{k}\left(\gamma_{(m)}-\gamma_{0}\right)\right)-f_{k, t-1}(0)\right] \boldsymbol{R}_{k, m \bullet}^{\prime} \boldsymbol{z}_{t-1} \boldsymbol{z}_{t-1}^{\prime} \boldsymbol{R}_{k}\right)\right\| \\
& \leq \frac{2}{T} \sum_{m=1}^{M} \sum_{t=1}^{T} \sum_{k=1}^{K} \mathrm{E}\left(\left|f_{k, t-1}\left(\boldsymbol{z}_{t-1}^{\prime} \boldsymbol{R}_{k}\left(\gamma_{(m)}-\gamma_{0}\right)\right)-f_{k, t-1}(0)\right|\left\|\boldsymbol{R}_{k, m \bullet}^{\prime} \boldsymbol{z}_{t-1} \boldsymbol{z}_{t-1}^{\prime} \boldsymbol{R}_{k}\right\|\right) \\
& \leq \frac{2 L_{0}}{T} \sum_{m=1}^{M} \sum_{t=1}^{T} \sum_{k=1}^{K} \mathrm{E}\left(\left|\boldsymbol{z}_{t-1}^{\prime} \boldsymbol{R}_{k}\left(\gamma_{(m)}-\gamma_{0}\right)\right|\left\|\boldsymbol{R}_{k, m \bullet}^{\prime} \boldsymbol{z}_{t-1} \boldsymbol{z}_{t-1}^{\prime} \boldsymbol{R}_{k}\right\|\right) \\
& \leq 2 L_{0} M K\|\boldsymbol{R}\|^{3}\left\|\gamma-\gamma_{0}\right\| \sup _{t} \mathrm{E}\left\|\boldsymbol{z}_{t-1}\right\|^{3},
\end{aligned}
$$

where the second inequality follows from the Jensen inequality (by convexity of the norm) and the third inequality from the Lipschitz condition, i.e. Assumption 3.6(b). Hence $\left\|\mathcal{H}_{T}^{*}-\overline{\mathcal{H}}_{T}\right\|=O\left(\left\|\gamma-\gamma_{0}\right\|\right)$, and together with Equation (3.46) this implies

$$
\lambda_{T}(\gamma)=\lambda_{T}\left(\gamma_{0}\right)+\overline{\mathcal{H}}_{T}\left(\gamma-\gamma_{0}\right)+O\left(\left\|\gamma-\gamma_{0}\right\|^{2}\right)
$$




\section{Combining the Results}

It remains to combine the individual results into a full proof. We take Equation (3.32) and substitute the result in Equation (3.49) evaluated at $\widehat{\gamma}_{T}$ to obtain

$$
\frac{1}{\sqrt{T}} \sum_{t=1}^{T} \boldsymbol{\eta}_{t}\left(\gamma_{0}\right)+\overline{\mathcal{H}}_{T} \sqrt{T}\left(\gamma-\gamma_{0}\right)=O_{p}\left(\sqrt{T}\left\|\widehat{\gamma}_{T}-\gamma_{0}\right\|^{2}\right)
$$

where we have use that $\lambda_{T}\left(\gamma_{0}\right)=0$. The domination condition (N-3)(i) implies that $\sqrt{T}\left\|\widehat{\gamma}_{T}-\gamma_{0}\right\|=O_{p}(1)$. Our consistency result, Theorem 3.1, provides $\left\|\widehat{\gamma}_{T}-\gamma_{0}\right\|=o_{p}(1)$, thus the RHS of Equation (3.50) converges in probability to zero. Rearranging the terms gives

$$
\left(\boldsymbol{R}^{\prime} \overline{\boldsymbol{V}}_{T} \boldsymbol{R}\right)^{-1 / 2}\left(\boldsymbol{R}^{\prime} \overline{\boldsymbol{H}}_{T} \boldsymbol{R}\right) \sqrt{T}\left(\widehat{\gamma}_{T}-\gamma_{0}\right)=-\left(\boldsymbol{R}^{\prime} \overline{\boldsymbol{V}}_{T} \boldsymbol{R}\right)^{-1 / 2} \frac{1}{\sqrt{T}} \sum_{t=1}^{T} \boldsymbol{\eta}_{t}\left(\gamma_{0}\right)+o_{p}(1)
$$

where the RHS converges in distribution to a $\mathrm{N}\left(\mathbf{0}, \boldsymbol{I}_{M}\right)$ distributed random vector. The proof is complete.

\section{A.4 Proof of Theorem 3: Consistent Estimation of the Asymptotic Variance}

\section{Consistent Estimation of $\bar{V}_{T}$}

We need to proof $\left\|\widehat{\boldsymbol{V}}_{T}-\overline{\boldsymbol{V}}_{T}\right\|=o_{p}(1)$. We define the matrix $\boldsymbol{V}_{T}=\frac{1}{T} \sum_{t=1}^{T} \boldsymbol{\eta}_{t}\left(\gamma_{0}\right) \boldsymbol{\eta}_{t}\left(\gamma_{0}\right)^{\prime}$ and note that $\overline{\boldsymbol{V}}_{T}=\mathrm{E}\left(\boldsymbol{V}_{T}\right)$. In view of the triangle inequality the claim in the first line of this paragraph holds if both $\left\|\widehat{V}_{T}-V_{T}\right\|$ and $\left\|V_{T}-\bar{V}_{T}\right\|$ converge in probability to zero.

We start with $\left\|\widehat{\boldsymbol{V}}_{T}-\boldsymbol{V}_{T}\right\|$. The inequality $|\operatorname{sgn}(\hat{a}) \operatorname{sgn}(\hat{b})-\operatorname{sgn}(a) \operatorname{sgn}(b)| \leq \mid \operatorname{sgn}(\hat{a})-$ $\operatorname{sgn}(a)|+| \operatorname{sgn}(\hat{b})-\operatorname{sgn}(b) \mid$, the definition of $\hat{u}_{k t}$, and Equation (3.38) imply that

$$
\begin{aligned}
\left\|\widehat{\boldsymbol{V}}_{T}-\boldsymbol{V}_{T}\right\| & =\left\|\frac{1}{T} \sum_{t=1}^{T} \sum_{k_{1}=1}^{K} \sum_{k_{2}}^{K}\left[\operatorname{sgn}\left(\hat{u}_{k_{1} T}\right) \operatorname{sgn}\left(\hat{u}_{k_{2} t}\right)-\operatorname{sgn}\left(u_{k_{1} t}\right) \operatorname{sgn}\left(u_{k_{2} t}\right)\right] \boldsymbol{R}_{k_{1}}^{\prime} \boldsymbol{z}_{t-1} \boldsymbol{z}_{t-1}^{\prime} \boldsymbol{R}_{k_{2}}\right\| \\
& \leq \frac{4 K}{T}\|\boldsymbol{R}\|^{2} \sum_{t=1}^{T}\left\|\boldsymbol{z}_{t-1}\right\|^{2} \sum_{k=1}^{K} \mathbb{1}_{\left\{\left|u_{k t}\right|<\mid \boldsymbol{z}_{t-1}^{\prime} \boldsymbol{R}_{k}\left(\widehat{\gamma}_{T}-\gamma_{0}\right)\right\}}:=A_{1 T} .
\end{aligned}
$$

We have $\operatorname{Pr}\left(\left\|\widehat{\boldsymbol{V}}_{T}-\boldsymbol{V}_{T}\right\|>\varepsilon\right) \leq \operatorname{Pr}\left(A_{1 T}>\varepsilon\right)$. To bound the latter probability we make use of the elementary inequality $\operatorname{Pr}\left(A_{T}\right)=\operatorname{Pr}\left(A_{T} \cap B_{T}\right)+\operatorname{Pr}\left(A_{T} \cap B_{T}^{c}\right) \leq \operatorname{Pr}\left(A_{T} \cap B_{T}\right)+\operatorname{Pr}\left(B_{T}^{c}\right)$, 
with $A_{T}=\left\{A_{1 T}>\varepsilon\right\}$ and $B_{T}=\left\{\left\|\widehat{\gamma}_{T}-\gamma_{0}\right\|<\delta\right\}$ for some arbitrary $\delta$. Theorem 3.2 shows that $\sqrt{T}\left(\widehat{\gamma}_{T}-\gamma_{0}\right)=O_{p}(1)$ and hence $\operatorname{Pr}\left(B_{T}^{c}\right) \rightarrow 0$. For $\left\|\widehat{\gamma}_{T}-\gamma_{0}\right\|<\delta$, and by application of the Markov inequality

$$
\begin{aligned}
\operatorname{Pr}\left(A_{1 T}>\varepsilon\right) & \leq \frac{1}{\epsilon} \mathrm{E}\left|A_{1 T}\right| \leq \frac{4 K}{\epsilon T}\|\boldsymbol{R}\|^{2} \sum_{t=1}^{T} \sum_{k=1}^{K} \mathrm{E}\left(\int_{-\left\|\boldsymbol{z}_{t-1}^{\prime} \boldsymbol{R}_{k}\right\| \delta}^{\left\|\boldsymbol{z}_{t-1}^{\prime} \boldsymbol{R}_{k}\right\| \delta} f_{k, t}(x) d x\left\|\boldsymbol{z}_{t-1}\right\|^{2}\right) \\
& \leq \frac{8 f_{0} K^{2}}{\epsilon}\|\boldsymbol{R}\|^{3} \delta \sup _{t} \mathrm{E}\left\|\boldsymbol{z}_{t-1}\right\|^{3},
\end{aligned}
$$

which can be made arbitrary small by choosing $\delta$ small enough.

We now shift our attention to $\left\|\boldsymbol{V}_{T}-\overline{\boldsymbol{V}}_{T}\right\|=\left\|\frac{1}{T} \sum_{t=1}^{T}\left[\boldsymbol{\eta}_{t}\left(\gamma_{0}\right) \boldsymbol{\eta}_{t}\left(\gamma_{0}\right)^{\prime}-\mathrm{E} \boldsymbol{\eta}_{t}\left(\gamma_{0}\right) \boldsymbol{\eta}_{t}\left(\gamma_{0}\right)^{\prime}\right]\right\|$. Let us first consider the $m^{\prime}$ th component of $\boldsymbol{\eta}_{t}\left(\gamma_{0}\right)$, i.e. $-\sum_{k=1}^{K} \boldsymbol{R}_{k, m \bullet}^{\prime} \boldsymbol{z}_{t-1} \operatorname{sgn}\left(u_{k t}\right)$. Now

$$
\begin{aligned}
\| \sum_{k=1}^{K} \boldsymbol{R}_{k, m}^{\prime} \boldsymbol{z}_{t-1} \operatorname{sgn}\left(u_{k t}\right) & -\mathrm{E}\left(\sum_{k=1}^{K} \boldsymbol{R}_{k, m}^{\prime} \boldsymbol{z}_{t-1} \operatorname{sgn}\left(u_{k t}\right) \mid \mathcal{F}_{t-m}^{t+m}\right) \|_{p} \\
& \leq K\|\boldsymbol{R}\|\left\|\boldsymbol{z}_{t-1}-\mathrm{E}\left(\boldsymbol{z}_{t-1} \mid \mathcal{F}_{t-m}^{t+m}\right)\right\|_{p}
\end{aligned}
$$

showing that the $m$ 'th component of $\boldsymbol{\eta}_{t}\left(\gamma_{0}\right)$ is near-epoch dependent in $L_{p}$ norm on $\left\{\boldsymbol{u}_{t}\right\}$ if $\boldsymbol{z}_{t-1}$ is near-epoch dependent on $\left\{\boldsymbol{u}_{t}\right\}$. The latter was shown in section 3.A.2. Corollary 4.3b of Gallant and White (1988) is applicable because $\left\|\boldsymbol{\eta}_{t}\left(\gamma_{0}\right)\right\|_{2 r} \leq D\|\boldsymbol{R}\| \sup _{t}\left\|\boldsymbol{z}_{t-1}\right\|<$ $\infty$ implies that any component of $\left\{\boldsymbol{\eta}_{t}\left(\gamma_{0}\right) \boldsymbol{\eta}_{t}\left(\gamma_{0}\right)^{\prime}-\mathrm{E} \boldsymbol{\eta}_{t}\left(\gamma_{0}\right) \boldsymbol{\eta}_{t}\left(\gamma_{0}\right)^{\prime}\right\}$ is near epoch dependent on $\left\{\boldsymbol{u}_{t}\right\}$ of arbitrary size. Together with the mixing conditions in Assumption 3.2(a) the result follows from the LLN for mixingales as provided by McLeish (1975).

\section{Consistent Estimation of $\overline{\boldsymbol{H}}_{T}$}

A first application of the triangle inequality gives

$$
\begin{aligned}
\left\|\widehat{H}_{T}-\bar{H}_{T}\right\| & \leq \sum_{k=1}^{K}\left\|\frac{2}{T} \sum_{t=1}^{T}\left(\frac{1}{2 \hat{c}_{k, T}} \mathbb{1}_{\left\{\left|\hat{u}_{k t}\right|<\hat{c}_{k, T}\right\}} \boldsymbol{R}_{k}^{\prime} \boldsymbol{z}_{t-1} \boldsymbol{z}_{t-1}^{\prime} \boldsymbol{R}_{k}-\mathrm{E} f_{k, t}(0) \boldsymbol{R}_{k}^{\prime} z_{t-1} \boldsymbol{z}_{t-1}^{\prime} \boldsymbol{R}_{k}\right)\right\| \\
& :=\sum_{k=1}^{K} \mathcal{E}_{k} .
\end{aligned}
$$

Equation (3.55) shows that the error in approximating $\bar{H}_{T}$ by $\widehat{H}_{T}$ can be attributed to the individual cross-sections. The error due to the $k^{\prime}$ th cross-section is denoted by $\mathcal{E}_{k}$ $(k \in\{1,2, \ldots, K\})$. To show $\left\|\widehat{H}_{T}-\bar{H}_{T}\right\|=o_{p}(1)$, it suffices to show that $\mathcal{E}_{k} \stackrel{p}{\longrightarrow} 0$ for every 
$k$. We now focus on an arbitrary $k$. Subsequent applications of the triangle inequality further decompose the approximation error into

$$
\begin{aligned}
& \mathcal{E}_{k} \leq \| \frac{1}{\hat{c}_{k, T} T} \sum_{t=1}^{T}\left(\mathbb{1}_{\left\{\left|\hat{u}_{k t}\right|<\hat{c}_{k, T}\right\}}-\mathbb{1}_{\left\{\left|u_{k t}\right|<\hat{c}_{k, T}\right\}}\right) \boldsymbol{R}_{k}^{\prime} \boldsymbol{z}_{t-1} \boldsymbol{z}_{t-1}^{\prime} \boldsymbol{R}_{k} \| \\
&+\left\|\frac{1}{T} \sum_{t=1}^{T}\left(\frac{1}{\hat{c}_{k, T}} \mathbb{1}_{\left\{\left|u_{k t}\right|<\hat{c}_{k, T}\right\}}-\frac{1}{c_{k, T}} \mathbb{1}_{\left\{\left|u_{k t}\right|<c_{k, T}\right\}}\right) \boldsymbol{R}_{k}^{\prime} \boldsymbol{z}_{t-1} \boldsymbol{z}_{t-1}^{\prime} \boldsymbol{R}_{k}\right\| \\
&+\| \frac{1}{c_{k, T} T} \sum_{t=1}^{T}\left(\mathbb{1}_{\left\{\left|u_{k t}\right|<c_{k, T}\right\}} \boldsymbol{R}_{k}^{\prime} \boldsymbol{z}_{t-1} \boldsymbol{z}_{t-1}^{\prime} \boldsymbol{R}_{k}-\mathrm{E}\left(\mathbb{1}_{\left\{\left|u_{k t}\right|<c_{k, T}\right\}} \boldsymbol{R}_{k}^{\prime} \boldsymbol{z}_{t-1} \boldsymbol{z}_{t-1}^{\prime} \boldsymbol{R}_{k}\right) \|\right. \\
&+\left\|\frac{2}{T} \sum_{t=1}^{T} \mathrm{E}\left(\left(\frac{1}{2 c_{k, T}} \mathrm{E}\left(\mathbb{1}_{\left\{\left|u_{k t}\right|<c_{k, T}\right\}} \mid \mathcal{F}_{t-1}\right)-f_{k, t}(0)\right) \boldsymbol{R}_{k}^{\prime} z_{t-1} \boldsymbol{z}_{t-1}^{\prime} \boldsymbol{R}_{k}\right)\right\| \\
&:=A_{k, T}^{(1)}+A_{k, T}^{(2)}+A_{k, T}^{(3)}+A_{k, T}^{(4)} .
\end{aligned}
$$

We will show that $A_{k, T}^{(1)}, A_{k, T}^{(2)}, A_{k, T}^{(3)}$, and $A_{k, T}^{(4)}$ all converge to zero in probability. Define the events $S_{k, T}^{(1)}=\left\{1-\delta_{1}<\frac{\hat{c}_{k, T}}{c_{k, T}}<1+\delta_{1}\right\}$ and $S_{k, T}^{(2)}=\left\{\left\|\widehat{\gamma}_{T}-\gamma_{0}\right\| \leq \delta_{2} c_{k, T}\right\}$. As in the proof above, we will consider the intersection ${ }^{15}$ with these event in combination with Markov's inequality. If both $S_{k, T}^{(1)}$ and $S_{k, T}^{(2)}$ occur, then

$$
\begin{aligned}
\left|\mathbb{1}_{\left\{\left|\hat{u}_{k t}\right|<c_{T}\right\}}-\mathbb{1}_{\left\{\left|u_{k t}\right|<\hat{c}_{T}\right\}}\right| & \leq \mathbb{1}_{\left\{\hat{c}_{k, T}-\boldsymbol{z}_{t-1}^{\prime} \boldsymbol{R}_{k}\left(\widehat{\boldsymbol{\gamma}}_{T}-\boldsymbol{\gamma}_{0}\right) \leq\left|u_{k t}\right| \leq \hat{c}_{k, T}+\boldsymbol{z}_{t-1}^{\prime} \boldsymbol{R}_{k}\left(\widehat{\gamma}_{T}-\boldsymbol{\gamma}_{0}\right)\right\}} \\
& \leq \mathbb{1}_{\left\{c_{k, T}\left(1-\delta_{1}\right)-\boldsymbol{z}_{t-1}^{\prime} \boldsymbol{R}_{k}\left(\widehat{\gamma}_{T}-\boldsymbol{\gamma}_{0}\right) \leq\left|u_{k t}\right| \leq c_{k, T}\left(1+\delta_{1}\right)+\boldsymbol{z}_{t-1}^{\prime} \boldsymbol{R}_{k}\left(\widehat{\gamma}_{T}-\boldsymbol{\gamma}_{0}\right)\right\}} \\
& \leq \mathbb{1}_{\left\{c_{k, T}\left(1-\delta_{1}\right)-\left\|\boldsymbol { z } _ { t - 1 } | \| | | \boldsymbol { R } \| \delta _ { 2 } c _ { k , T } \leq | u _ { k t } \left|\leq c_{k, T}\left(1+\delta_{1}\right)+\left\|\boldsymbol{z}_{t-1}\left|\|\mid \boldsymbol{R}\| \delta_{2} c_{k, T}\right\}\right.\right.\right.\right.} .
\end{aligned}
$$

The first inequality follows from the definition of $\hat{u}_{k t}$. The second and third inequality rely on the occurrence of the events $S_{k, T}^{(1)}$ and $S_{k, T}^{(2)}$, respectively. Equation (3.57) and Assumption 3.6(a) make sure that $\mathrm{E}\left(\left|\mathbb{1}_{\left\{\left|\hat{u}_{k t}\right|<c_{k, T}\right\}}-\mathbb{1}_{\left\{\left|u_{k t}\right|<\hat{c}_{k, T}\right\}}\right| \mid \mathcal{F}_{t-1}\right) \leq 2 f_{0} c_{k, T}\left(\delta_{1}+\delta_{2}\left\|\boldsymbol{z}_{t-1}|\|\mid \boldsymbol{R}\|)\right.\right.$. We conclude

$$
\begin{aligned}
& \operatorname{Pr}\left(A_{k, T}^{(1)}>\varepsilon\right) \\
& \leq \operatorname{Pr}\left(\left\{\frac{1}{c_{k, T} T}\|\boldsymbol{R}\|^{2} \sum_{t=1}^{T}\left\|\boldsymbol{z}_{t-1}\right\|^{2} \sum_{k=1}^{K}\left|\mathbb{1}_{\left\{\left|\hat{u}_{k t}\right|<\hat{c}_{k, T}\right\}}-\mathbb{1}_{\left\{\left|u_{k t}\right|<\hat{c}_{k, T}\right\}}\right|>\varepsilon\right\} \cap S_{k, T}^{(1)} \cap S_{k, T}^{(2)}\right) \\
& \quad+\operatorname{Pr}\left(\left\{S_{k, T}^{(1)}\right\}^{c}\right)+\operatorname{Pr}\left(\left\{S_{k, T}^{(2)}\right\}^{c}\right) \\
& \leq \frac{2 f_{0} K}{\varepsilon}\|\boldsymbol{R}\|^{2}\left(\delta_{1} \sup _{t} \mathrm{E}\left\|\boldsymbol{z}_{t-1}\right\|^{2}+\delta_{2}\|\boldsymbol{R}\| \sup _{t} \mathrm{E}\left\|\boldsymbol{z}_{t-1}\right\|^{3}\right)+\operatorname{Pr}\left(\left\{S_{k, T}^{(1)}\right\}^{c}\right)+\operatorname{Pr}\left(\left\{S_{k, T}^{(2)}\right\}^{c}\right)
\end{aligned}
$$

${ }^{15}$ Specifically, we will use $\operatorname{Pr}(A)=\operatorname{Pr}(A \cap(B \cap C))+\operatorname{Pr}\left(A \cap(B \cap C)^{c}\right)=\operatorname{Pr}(A \cap B \cap C)+\operatorname{Pr}\left(\left(A \cap B^{c}\right) \cup\left(A \cap C^{c}\right)\right) \leq$ $\operatorname{Pr}(A \cap B \cap C)+\operatorname{Pr}\left(A \cap B^{c}\right)+\operatorname{Pr}\left(A \cap C^{c}\right) \leq \operatorname{Pr}(A \cap B \cap C)+\operatorname{Pr}\left(B^{c}\right)+\operatorname{Pr}\left(C^{c}\right)$. 
by the Markov inequality. Now let us consider the three terms in more detail. The first term can be made as small as necessary by taking $\delta_{1}$ and $\delta_{2}$ small enough. $S_{k, T}^{(1)}$ will occur with arbitrarily high probability because we assumed $\hat{c}_{k, T} / c_{k, T} \stackrel{p}{\longrightarrow} 1$, see Assumption 3.8(a). Its complement will thus occur with arbitrary low probability. Similarly, Assumption 3.8(c) and Theorem 3.2 imply that $\frac{1}{c_{k, T} \sqrt{T}} \sqrt{T}\left\|\widehat{\gamma}_{T}-\gamma_{0}\right\|=o_{p}(1)$ and therefore the probability of $\left\{S_{k, T}^{(2)}\right\}^{c}$ is sufficiently small for large $T$.

The term $A_{k, T}^{(2)}$ should become arbitrarily small because $\hat{c}_{k, T} / c_{k, T} \stackrel{p}{\longrightarrow} 1$. As such we only consider the occurrence of $S_{k, T}^{(1)}$. If the event $S_{k, T}^{(1)}=\left\{1-\delta<\frac{\hat{c}_{k, T}}{c_{k, T}}<1+\delta_{1}\right\}$ occurs, then

$$
\begin{aligned}
\left|\frac{1}{\hat{c}_{T}} \mathbb{1}_{\left\{\left|u_{k t}\right|<\hat{c}_{k, T}\right\}}-\frac{1}{c_{T}} \mathbb{1}_{\left\{\left|u_{k t}\right|<c_{k, T}\right\}}\right| \leq \frac{1}{c_{k, T}\left(1-\delta_{1}\right)} \mathbb{1}_{\left\{\left|u_{k t}\right|<\left(1+\delta_{1}\right) c_{k, T}\right\}}-\frac{1}{c_{k, T}} \mathbb{1}_{\left\{\left|u_{k t}\right|<c_{k, T}\right\}} \\
\leq \frac{\delta_{1}}{c_{T}\left(1-\delta_{1}\right)} \mathbb{1}_{\left.\left\{\left|u_{k t}\right|<\left(1+\delta_{1}\right) c_{k, T}\right)\right\}}+\frac{1}{c_{k, T}}\left(\mathbb{1}_{\left.\left\{\left|u_{k t}\right|<\left(1+\delta_{1}\right) c_{k, T}\right)\right\}}-\mathbb{1}_{\left\{\left|u_{k t}\right|<c_{k, T}\right\}}\right) .
\end{aligned}
$$

The conditional expectation of this final expression is bounded above by $\frac{2 f_{0} \delta_{1}\left(1+\delta_{1}\right)}{1-\delta_{1}}+2 f_{0} \delta_{1}$ in view of Assumption 3.6(a). The Markov inequality yields

$$
\begin{aligned}
\operatorname{Pr}\left(A_{k, T}^{(2)}>\varepsilon\right) \leq & \operatorname{Pr}\left(\left\{\frac{1}{T}\|\boldsymbol{R}\|^{2} \sum_{t=1}^{T}\left\|z_{t-1}\right\|^{2} \sum_{k=1}^{K}\left|\frac{1}{\hat{c}_{k, T}} \mathbb{1}_{\left\{\left|u_{k t}\right|<\hat{c}_{k, T}\right\}}-\frac{1}{c_{k, T}} \mathbb{1}_{\left\{\left|u_{k t}\right|<c_{k, T}\right\}}\right|>\varepsilon\right\} \cap S_{k, T}^{(1)}\right) \\
& \quad+\operatorname{Pr}\left(\left\{S_{k, T}^{(1)}\right\}^{c}\right) \\
\leq & \frac{2 f_{0} K}{\varepsilon}\|\boldsymbol{R}\|^{2}\left(\frac{\delta_{1}\left(1+\delta_{1}\right)}{1-\delta_{1}}\right) \sup _{t} \mathrm{E}\left\|z_{t-1}\right\|^{2}+\operatorname{Pr}\left(\left\{S_{k, T}^{(1)}\right\}^{c}\right) .
\end{aligned}
$$

And by similar arguments as before, $A_{k, T}^{(2)} \stackrel{p}{\longrightarrow} 0$ follows.

We proceed with $A_{k, T}^{(3)}$. We will use a LLN for mixingale triangular arrays to show that the difference between the two matrices converges to zero. For $m_{1}, m_{2} \in\{1,2, \ldots, M\}$ we focus on the $\left(m_{1}, m_{2}\right)$ element of these matrices and define the scalar $X_{k, T, t, m_{1} m_{2}}=$ $\frac{1}{c_{k, T}}\left(\mathbb{1}_{\left\{\left|u_{k t}\right|<c_{k, T}\right\}} \boldsymbol{R}_{k, m_{1}}^{\prime} \boldsymbol{z}_{t-1} \boldsymbol{z}_{t-1}^{\prime} \boldsymbol{R}_{k, m_{2}} \bullet-\mathrm{E}\left(\mathbb{1}_{\left\{\left|u_{k t}\right|<c_{k, T}\right\}} \boldsymbol{R}_{k, m_{1}}^{\prime} \bullet \boldsymbol{z}_{t-1} \boldsymbol{z}_{t-1}^{\prime} \boldsymbol{R}_{k, m_{2}} \bullet\right)\right.$. $X_{k, T, t, m_{1} m_{2}}$ is a mean zero triangular array due to the presence of the sample size $T$ in the bandwidth 
$c_{k, T}$. But $u_{k t}$ is $\mathcal{F}_{t-m}^{t+m}$-measurable for any $m \geq 0$, and hence

$$
\begin{aligned}
\| X_{k, T, t, m_{1} m_{2}}-\mathrm{E} & \left(X_{k, T t, m_{1} m_{2}} \mid \mathcal{F}_{t-m}^{t+m}\right) \|_{1} \\
& =\left\|\frac{1}{c_{k, T}} \mathbb{1}_{\left\{\left|u_{k t}\right|<c_{k, T}\right\}} \boldsymbol{R}_{k, m_{1}}^{\prime} \cdot\left[\boldsymbol{z}_{t-1} \boldsymbol{z}_{t-1}^{\prime}-\mathrm{E}\left(\boldsymbol{z}_{t-1} \boldsymbol{z}_{t-1}^{\prime} \mid \mathcal{F}_{t-m}^{t+m}\right)\right] \boldsymbol{R}_{k, m_{2}} \cdot\right\|_{1} \\
& \leq 2 K^{3}\|\boldsymbol{R}\|^{2}\left(\max _{k=1, \ldots, K} f_{k}^{*}\right)\left\|\boldsymbol{z}_{t-1} \boldsymbol{z}_{t-1}^{\prime}-\mathrm{E}\left(\boldsymbol{z}_{t-1} \boldsymbol{z}_{t-1}^{\prime} \mid \mathcal{F}_{t-m}^{t+m}\right)\right\|_{1} .
\end{aligned}
$$

Equation (3.61) shows that $X_{k, T, t, m_{1} m_{2}}$ is $L_{1}$-NED on $\left\{\boldsymbol{u}_{t}\right\}$ and therefore an $L_{1}$ mixingale. The moment conditions in Assumption 3.2(b) ensure uniform integrability of $X_{k, T, t, m_{1} m_{2}}$. The claim that $A_{k, T}^{(3)} \stackrel{p}{\longrightarrow} 0$ follows by a weak LLN for triangular $L_{1}$-mixingale arrays, i.e. Theorem 2 of Andrews (1988).

$A_{k, T}^{(4)}$ is a deterministic, $\frac{1}{2 c_{k, T}} \mathrm{E}\left(\mathbb{1}_{\left\{\left|u_{k t}\right|<c_{k, T}\right\}} \mid \mathcal{F}_{t-1}\right)=\frac{\operatorname{Pr}\left(u_{k t} \leq c_{k, T} \mid \mathcal{F}_{t-1}\right)-\operatorname{Pr}\left(u_{k t}<-c_{k, T} \mid \mathcal{F}_{t-1}\right)}{2 c_{k, T}}$ is a symmetric difference quotient, and $c_{k, T} \rightarrow 0$ by Assumption 3.8(a). But these imply that $\frac{1}{2 c_{k, T}} \mathrm{E}\left(\mathbb{1}_{\left\{\left|u_{k t}\right|<c_{k, T}\right\}} \mid \mathcal{F}_{t-1}\right) \rightarrow f_{k, t}(0)$ for large $T$. The unconditional expectation under the summation signs is dominated by $f_{0}\|R\|^{2} \sup _{t} \mathrm{E}\left\|\boldsymbol{z}_{t-1}\right\|^{2}<\infty$. $A_{k, T}^{(3)}$ therefore converges in probability to zero by Lebesgue's dominated convergence theorem.

\section{A.5 Proof of Theorem 4-6: Test Statistics}

\section{Wald Test}

The limiting distribution of the unrestricted estimator $\boldsymbol{b}_{U}$ is obtained from Theorem 3.2 by choosing $\boldsymbol{R}=\boldsymbol{I}_{K(K p+1)}$, hence $\overline{\boldsymbol{V}}_{T}^{-1 / 2} \overline{\boldsymbol{H}}_{T} \sqrt{T}\left(\widehat{\boldsymbol{b}}_{T}^{U}-\boldsymbol{b}\right) \stackrel{d}{\longrightarrow} \mathrm{N}\left(\mathbf{0}, \boldsymbol{I}_{K(K p+1)}\right)$. Under the null hypothesis $H_{0}: \boldsymbol{C b}=\boldsymbol{c}$, we have thus $\left(\boldsymbol{C} \overline{\boldsymbol{H}}_{T}^{-1} \overline{\boldsymbol{V}}_{T} \overline{\boldsymbol{H}}_{T}^{-1} \boldsymbol{C}^{\prime}\right)^{-1 / 2} \sqrt{T}\left(\widehat{C}_{T}^{U}-\boldsymbol{c}\right) \stackrel{d}{\longrightarrow}$

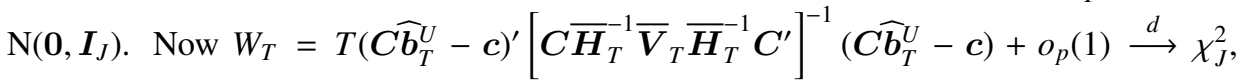
because the matrices $\overline{\boldsymbol{V}}_{T}$ and $\overline{\boldsymbol{H}}_{T}$ are consistently estimated in view of Theorem 3.3. The limiting distribution of the Wald test statistic is established.

\section{LM Test}

We consider the quantity $Z_{T}\left(\tau, \gamma_{0}\right)$ of Appendix 3.B, and combine with Equation (3.49) to give

$$
Z_{T}\left(\boldsymbol{\tau}, \gamma_{0}\right)=\frac{\left\|\frac{1}{\sqrt{T}} \sum_{t=1}^{T}\left[\boldsymbol{\eta}_{t}(\boldsymbol{\tau})-\boldsymbol{\eta}_{t}\left(\gamma_{0}\right)-\overline{\mathcal{H}}_{T} \sqrt{T}\left(\boldsymbol{\tau}-\gamma_{0}\right)+O\left(\sqrt{T}\left\|\boldsymbol{\tau}-\gamma_{0}\right\|^{2}\right)\right]\right\|}{1+\left\|\overline{\mathcal{H}}_{T} \sqrt{T}\left(\boldsymbol{\tau}-\gamma_{0}\right)+O\left(\sqrt{T}\left\|\boldsymbol{\tau}-\gamma_{0}\right\|^{2}\right)\right\|}
$$


For an arbitrary $M$, we can make sure that $M / \sqrt{T}<d_{0}$ by taking a large enough $T$. Lemma 3.1 from Appendix 3.B now shows that $\sup _{\sqrt{T}\left\|\tau-\gamma_{0}\right\|<M} Z_{T}\left(\tau, \gamma_{0}\right)=o_{p}(1)$. Up to this moment we have use the parametrization in terms in $\gamma$ and as such allowed the parameters estimates to be restricted. For the Score test statistic we need the unrestricted score. We change the notation according and replace $\boldsymbol{\tau}$ and $\gamma_{0}$ by $\boldsymbol{b}$ and $\boldsymbol{b}_{0}$, respectively. Also note that we have $\frac{1}{\sqrt{T}} \sum_{t=1}^{T} \boldsymbol{\eta}_{t}(\boldsymbol{b})=\boldsymbol{g}_{T}(\boldsymbol{b})$ once $\boldsymbol{\eta}_{t}$ is based on the unrestricted estimates. Therefore, from Equation (3.62) and Lemma 3.1 of Appendix B,

$$
\sup _{\left\|\sqrt{T}\left(\boldsymbol{b}-\boldsymbol{b}_{0}\right)\right\| \leq M} \frac{\left\|-\boldsymbol{g}_{T}(\boldsymbol{b})+\boldsymbol{g}_{T}\left(\boldsymbol{b}_{0}\right)-\overline{\mathcal{H}}_{T} \sqrt{T}\left(\boldsymbol{b}-\boldsymbol{b}_{0}\right)+O\left(\sqrt{T}\left\|\boldsymbol{b}-\boldsymbol{b}_{0}\right\|^{2}\right)\right\|}{1+\left\|\overline{\mathcal{H}}_{T} \sqrt{T}\left(\boldsymbol{b}-\boldsymbol{b}_{0}\right)+O\left(\sqrt{T}\left\|\boldsymbol{b}-\boldsymbol{b}_{0}\right\|^{2}\right)\right\|}=o_{p}(1) .
$$

The event $\left\{\sqrt{T}\left(\widehat{b}_{T}^{R}-\boldsymbol{b}_{0}\right) \leq M\right\}$ will occur with arbitrary probability for large enough $M$, so upon setting $\boldsymbol{b}=\widehat{\boldsymbol{b}}_{T}^{R}$ we arrive at $\left.\boldsymbol{g}_{T} \widehat{\boldsymbol{b}}_{T}^{R}\right)-\boldsymbol{g}_{T}\left(\boldsymbol{b}_{0}\right)+\overline{\boldsymbol{H}}_{T} \sqrt{T}\left(\widehat{\boldsymbol{b}}_{T}^{R}-\boldsymbol{b}_{0}\right)=o_{p}(1)$. The outer vectors in the quadratic form of the Score test statistic $S_{T}$ will now equal

$$
\begin{aligned}
\left.\boldsymbol{C} \widehat{\boldsymbol{H}}_{T}^{-1} \boldsymbol{g}_{T} \widehat{\boldsymbol{b}}_{T}^{R}\right) \\
=\boldsymbol{C} \widehat{\boldsymbol{H}}_{T}^{-1}\left(\boldsymbol{g}_{T}\left(\boldsymbol{b}_{0}\right)-\overline{\boldsymbol{H}}_{T} \sqrt{T}\left(\widehat{\boldsymbol{b}}_{T}^{R}-\boldsymbol{b}_{0}\right)\right)+o_{p}(1) \\
=\boldsymbol{C}\left[\overline{\boldsymbol{H}}_{T}^{-1}+\left(\widehat{\boldsymbol{H}}_{T}^{-1}-\overline{\boldsymbol{H}}_{T}^{-1}\right)\right]\left(\boldsymbol{g}_{T}\left(\boldsymbol{b}_{0}\right)-\overline{\boldsymbol{H}}_{T} \sqrt{T}\left(\widehat{\boldsymbol{b}}_{T}^{R}-\boldsymbol{b}_{0}\right)\right)+o_{p}(1) \\
=\boldsymbol{C} \overline{\boldsymbol{H}}_{T}^{-1} \boldsymbol{g}_{T}\left(\boldsymbol{b}_{0}\right)-\sqrt{T} \boldsymbol{C}\left(\widehat{\boldsymbol{b}}_{T}^{R}-\boldsymbol{b}_{0}\right)+o_{p}(1)=\boldsymbol{C} \overline{\boldsymbol{H}}_{T}^{-1} \boldsymbol{g}_{T}\left(\boldsymbol{b}_{0}\right)+o_{p}(1),
\end{aligned}
$$

where the third equality uses the consistent estimation of the matrix $\overline{\boldsymbol{H}}_{T}$, and the final equality uses the fact that the constraint estimator satisfies $\boldsymbol{C}\left(\widehat{\boldsymbol{b}}_{T}^{R}-\boldsymbol{b}_{0}\right)=\mathbf{0}$. The standardization in the quadratic form is thus the inverse of the asymptotic covariance matrix. The chi-squared limiting distribution follows.

\section{LR Test}

Proofs concerning the LR test statistic typically involve a reparameterization (e.g. Jaeckel (1972), Koenker and Bassett (1982), and Koenker and Machado (1999)). We introduced the rescaled and centered parameter vector $\boldsymbol{\delta}=\sqrt{T}\left(\boldsymbol{b}-\boldsymbol{b}_{0}\right)$, and transform functions $\boldsymbol{g}_{T}$ and $V$ accordingly to ${ }^{16}$

$$
\tilde{\boldsymbol{g}}_{T}(\boldsymbol{\delta})=\boldsymbol{g}_{T}\left(\boldsymbol{b}_{0}+\frac{\boldsymbol{\delta}}{\sqrt{T}}\right)=-\frac{1}{\sqrt{T}} \sum_{t=1}^{T}\left(\operatorname{sgn}\left(\boldsymbol{u}_{t}-\left(\boldsymbol{I}_{k} \otimes \boldsymbol{z}_{t-1}^{\prime}\right) \frac{\boldsymbol{\delta}}{\sqrt{T}}\right) \otimes \boldsymbol{I}_{K p+1}\right) \boldsymbol{z}_{t-1},
$$

\footnotetext{
${ }^{16}$ Note that the transformed functions are identical to the original ones. They are only expressed in different variables, as an example we have $\tilde{\boldsymbol{g}}_{T}(\mathbf{0})=\boldsymbol{g}_{T}\left(\boldsymbol{b}_{0}\right)$.
} 
Equation 3.63 reads $\sup _{\|\boldsymbol{\delta}\| \leq M}\left\|\tilde{\boldsymbol{g}}_{T}(\boldsymbol{\delta})-\tilde{\boldsymbol{g}}_{T}(\mathbf{0})+\overline{\boldsymbol{H}}_{T} \boldsymbol{\delta}\right\|=o_{p}(1)$ in this new parametrization. This result states that the distance between the score $\tilde{\boldsymbol{g}}_{T}$ and the gradient of the quadratic form $\tilde{Q}(\delta)=\frac{1}{2} \boldsymbol{\delta}^{\prime} \overline{\boldsymbol{H}}_{T} \boldsymbol{\delta}-\boldsymbol{\delta}^{\prime} \tilde{\boldsymbol{g}}_{T}(\mathbf{0})+V(\mathbf{0})$ is asymptotically negligible. From Lemma 1 of Jaeckel (1972) we can conclude that $\sup _{\|\delta\| \leq M}|V(\boldsymbol{\delta})-\tilde{Q}(\boldsymbol{\delta})|=o_{p}(1) .{ }^{17}$ We first develop the unconstrained estimator. Let $\widehat{\delta}_{T}^{U}$ denote the minimizer of $V(\delta)$, and $\widehat{\delta}_{T, 0}^{U}$ the minimizer of the quadratic form $\tilde{Q}(\boldsymbol{\delta})$, hence $\widehat{\delta}_{T, 0}^{U}=\overline{\boldsymbol{H}}_{T}^{-1} \tilde{\boldsymbol{g}}_{T}(\mathbf{0})$. The set $\{\boldsymbol{\delta} \mid\|\boldsymbol{\delta}\| \leq M\}$ is closed and bounded (hence compact). Together with the convexity result on page 193 of Pollard (1991), this implies that $\widehat{\delta}_{T, 0}^{U}=\widehat{\delta}_{T, 0}^{U}+o_{p}(1)$. Overall, we have

$$
V\left(\widehat{\boldsymbol{b}}_{T}^{R}\right)=\tilde{V}\left(\widehat{\delta}_{T}^{U}\right)=\left[\tilde{V}\left(\widehat{\delta}_{T}^{U}\right)-\tilde{Q}\left(\widehat{\delta}_{T}^{U}\right)\right]+\left[\tilde{Q}\left(\widehat{\delta}_{T}^{U}\right)-\tilde{Q}\left(\widehat{\delta}_{T, 0}^{U}\right)\right]+\tilde{Q}\left(\widehat{\delta}_{T, 0}^{U}\right)=\tilde{Q}\left(\widehat{\delta}_{T, 0}^{U}\right)+o_{p}(1)
$$

where the first term vanishes because the quadratic approximation becomes more and more accurate as $T$ increases, and the second term vanishes in view of the continuous mapping theorem. Similarly, we obtain $V\left(\widehat{\boldsymbol{b}}_{T}^{R}\right)=\tilde{Q}\left(\widehat{\delta}_{T, 0}^{R}\right)+o_{p}(1)$ for the restricted estimator. The minimizer of the restricted quadratic form should satisfy $\widehat{C} \widehat{\delta}_{T, 0}^{R}=\mathbf{0}$, because $\widehat{C b}_{T}^{R}=\boldsymbol{b}_{0}$ under the null.

The minimizer of the restricted quadratic form can be found by minimization of the Lagrangian with Lagrange multipliers $\boldsymbol{\zeta}$ equals $\mathscr{L}(\boldsymbol{\delta} ; \boldsymbol{\eta})=\frac{1}{2} \boldsymbol{\delta}^{\prime} \overline{\boldsymbol{H}}_{T} \boldsymbol{\delta}-\boldsymbol{\delta}^{\prime} \tilde{\boldsymbol{g}}_{T}(\mathbf{0})+V(0)+$ $\boldsymbol{\zeta}^{\prime} \boldsymbol{C} \boldsymbol{\delta}$. The first order condition provides

$$
\left[\begin{array}{cc}
\overline{\boldsymbol{H}}_{T} & \boldsymbol{C}^{\prime} \\
\boldsymbol{C} & \mathbf{O}
\end{array}\right]\left[\begin{array}{c}
\widehat{\boldsymbol{\delta}}_{T, 0}^{R} \\
\boldsymbol{\zeta}
\end{array}\right]=\left[\begin{array}{c}
\tilde{\boldsymbol{g}}_{T}(\mathbf{0}) \\
\mathbf{0}
\end{array}\right],
$$

and by the results for partitioned matrix inverses

$$
\begin{aligned}
\boldsymbol{\zeta} & =\left[\boldsymbol{C} \overline{\boldsymbol{H}}_{T}^{-1} \boldsymbol{C}^{\prime}\right]^{-1} \boldsymbol{C} \overline{\boldsymbol{H}}_{T}^{-1} \boldsymbol{g}_{T}\left(\boldsymbol{b}_{0}\right)=\left[\boldsymbol{C} \overline{\boldsymbol{H}}_{T}^{-1} \boldsymbol{C}^{\prime}\right]^{-1} \boldsymbol{C} \widehat{\boldsymbol{\delta}}_{T, 0}^{U}, \\
\widehat{\boldsymbol{\delta}}_{T, 0}^{R} & =\overline{\boldsymbol{H}}_{T}^{-1} \boldsymbol{g}_{T}\left(\boldsymbol{b}_{0}\right)-\overline{\boldsymbol{H}}_{T}^{-1} \boldsymbol{C}^{\prime} \boldsymbol{\zeta}=\widehat{\boldsymbol{\delta}}_{T, 0}^{U}-\overline{\boldsymbol{H}}_{T}^{-1} \boldsymbol{C}^{\prime}\left[\boldsymbol{C} \overline{\boldsymbol{H}}_{T}^{-1} \boldsymbol{C}^{\prime}\right]^{-1} \boldsymbol{C} \widehat{\boldsymbol{\delta}}_{T, 0}^{U} .
\end{aligned}
$$

\footnotetext{
${ }^{17}$ The intuition behind the proof of Jaeckel (1972) is as follows. The sum of least absolute deviations, $\tilde{V}(\boldsymbol{\delta})$, and its quadratic approximation, $\tilde{Q}(\boldsymbol{\delta})$, clearly take the same value at $\boldsymbol{\delta}=\mathbf{0}$. If we move away from the origin, then the derivatives of $\tilde{V}(\boldsymbol{\delta})$ and the quadratic form are arbitrary close. This in turn forces $\tilde{V}(\boldsymbol{\delta})$ and $\tilde{Q}(\boldsymbol{\delta})$ to be close together.
} 
The difference in the quadratic forms can now be computed to obtain the desired result.

$$
\begin{aligned}
\tilde{V}\left(\widehat{\boldsymbol{\delta}}_{T}^{R}\right) & -\tilde{V}\left(\widehat{\boldsymbol{\delta}}_{T}^{U}\right) \\
& =\frac{1}{2} \widehat{\boldsymbol{\delta}}_{T, 0}^{R \prime} \overline{\mathcal{H}}_{T} \widehat{\boldsymbol{\delta}}_{T, 0}^{R}-\frac{1}{2} \widehat{\boldsymbol{\delta}}_{T, 0}^{U} \overline{\mathcal{H}}_{T} \widehat{\boldsymbol{\delta}}_{T, 0}^{U}-\left(\widehat{\boldsymbol{\delta}}_{T, 0}^{R}-\widehat{\boldsymbol{\delta}}_{T, 0}^{U}\right)^{\prime} \boldsymbol{g}_{T}\left(\boldsymbol{b}_{0}\right)+o_{p}(1) \\
& =-\boldsymbol{\zeta}^{\prime} \boldsymbol{C} \widehat{\boldsymbol{\delta}}_{T, 0}^{R}+\frac{1}{2} \boldsymbol{\zeta}^{\prime} \boldsymbol{C} \overline{\mathcal{H}}_{T}^{-1} \boldsymbol{C}^{\prime} \boldsymbol{\zeta}-\left(\widehat{\boldsymbol{\delta}}_{T, 0}^{R}-\widehat{\boldsymbol{\delta}}_{T, 0}^{U}\right)^{\prime} \boldsymbol{g}_{T}\left(\boldsymbol{b}_{0}\right)+o_{p}(1) \\
& =\frac{1}{2} \boldsymbol{\zeta}^{\prime} \boldsymbol{C} \overline{\mathcal{H}}_{T}^{-1} \boldsymbol{C}^{\prime} \boldsymbol{\zeta}+o_{p}(1)=\frac{1}{2} \boldsymbol{g}_{T}^{\prime}\left(\boldsymbol{b}_{0}\right) \overline{\mathcal{H}}_{T}^{-1} \boldsymbol{C}^{\prime}\left[\boldsymbol{C} \overline{\mathcal{H}}_{T}^{-1} \boldsymbol{C}^{\prime}\right]^{-1} \boldsymbol{C} \overline{\mathcal{H}}_{T}^{-1} \boldsymbol{g}_{T}\left(\boldsymbol{b}_{0}\right)+o_{p}(1) .
\end{aligned}
$$

From our previous CLT result, we know that $\left(\bar{V}_{T}\right)^{-1 / 2} \boldsymbol{g}_{T}\left(\boldsymbol{b}_{0}\right) \stackrel{d}{\longrightarrow} \mathrm{N}\left(\mathbf{0}, \boldsymbol{I}_{K(1+K p)}\right)$. The quadratic form in Equation (3.69) is thus $O_{p}(1)$, but not pivotal because the matrix in the quadratic form is not the inverse of the asymptotic covariance matrix.

\section{A.6 Proof of Theorem 7: Consistent Selection of the Lag Order}

We have to rule out the possibility to (1) select less lags than $p_{0}$, and (2) include additional lags which have parameters are zero. We consider both cases separately as in Machado (1993). We start by showing that the probability of under-selection is zero asymptotically. Consider a $p$ such that $0 \leq p<p_{0}$, the probability of under-selection is given by

$$
\operatorname{Pr}\left(\operatorname{Cr}(p)<\operatorname{Cr}\left(p_{0}\right)\right)=\operatorname{Pr}\left(2 K \log \left[F\left(\widehat{\boldsymbol{b}}_{T}^{(p)}\right) / F\left(\widehat{\boldsymbol{b}}_{T}^{\left(p_{0}\right)}\right)\right]<\left(p_{0}-p\right) K^{2} \frac{c_{T}}{T}\right) .
$$

Consider $F(\boldsymbol{b})=\frac{1}{K T} \sum_{t=1}^{T} \sum_{k=1}^{K}\left|y_{k t}-\boldsymbol{z}_{t-1}^{\prime} \boldsymbol{E}_{k} \boldsymbol{b}\right|$. Equation (3.26) (with $\boldsymbol{R}_{k}=\boldsymbol{E}_{k}$ and $\boldsymbol{r}_{k}=\mathbf{0}$ to have the unrestricted estimates) states that this expression converges uniformly to its expectation. The identifiability condition of Equation (3.27) subsequently shows that this expectation is uniquely minimized at the true parameter values. In other words, enforcing nonzero coefficient matrices to be zero (which is implicitly the case if we select $p<p_{0}$ ) creates a loss of fit, and $F\left(\widehat{\boldsymbol{b}}_{T}^{(p)}\right)>F\left(\widehat{\boldsymbol{b}}_{T}^{\left(p_{0}\right)}\right)$ holds almost surely. Based on Equation (3.70), we conclude that no under-selection will occur because $c_{T} / T \rightarrow 0$.

Now the case $p>p_{0}$. The probability to over-select the lag order is

$$
\operatorname{Pr}\left(\operatorname{Cr}(p)<\operatorname{Cr}\left(p_{0}\right)\right)=\operatorname{Pr}\left(2 K T \log \left[1+\frac{F\left(\widehat{\boldsymbol{b}}_{T}^{\left(p_{0}\right)}\right)-F\left(\widehat{\boldsymbol{b}}_{T}^{(p)}\right)}{F\left(\widehat{\boldsymbol{b}}_{T}^{(p)}\right)}\right]>\left(p-p_{0}\right) K^{2} c_{T}\right)
$$


The expression on the left of the inequality sign behaves as

$$
2 K T \frac{F\left(\widehat{\boldsymbol{b}}_{T}^{\left(p_{0}\right)}\right)-F\left(\widehat{\boldsymbol{b}}_{T}^{(p)}\right)}{F\left(\widehat{\boldsymbol{b}}_{T}^{(p)}\right)}=2 \frac{L R_{T}}{F\left(\widehat{\boldsymbol{b}}_{T}^{(p)}\right)}
$$

by the Taylor approximation $\log (1+x) \approx x . L R_{T}$ is the likelihood ratio test statistic to test the nullity of all coefficients in the matrices in front of lag $p_{0}+1, p_{0}+2, \ldots, p_{\max }$. It is bounded in view of Theorem 3.6. $F\left(\widehat{\boldsymbol{b}}_{T}^{(p)}\right)$ converges in probability to a positive number. The quantity in Equation (3.72) is thus $O_{p}(1)$. The condition $c_{T} \rightarrow \infty$ now avoids overselection. 


\section{B Supplementary Material}

\section{B.1 Huber's (1967) Lemma for Dependent Processes}

This addendum is concerned with the proof of Lemma 3 of Huber (1967) for dependent sequences. The original result from Huber (1967) assumed i.i.d. observations. Powell (1984) considered a censored regression setup in which the regressors $\left\{x_{t}\right\}$ are independent, but not necessarily identically distributed. The extension to $\alpha$-mixing processes is given in Weiss (1991). The derivation below follows Huber (1967) step-by-step and as such hopes to highlight the changes that are needed to deal with dependent sequences. The notation follows the main text. The mathematical arguments are completely due to the aforementioned authors.

As in Huber (1967), we define $\boldsymbol{\lambda}_{T}(\gamma)=\frac{1}{T} \sum_{t=1}^{T} \mathrm{E} \boldsymbol{\eta}_{t}(\gamma)$ and

$$
\zeta_{t}(\gamma, d)=\sup _{\|\tau-\gamma\| \leq d}\left\|\eta_{t}(\tau)-\eta_{t}(\gamma)\right\|
$$

The required assumptions are:

(N-1). For each fixed $\gamma \in \Gamma, \boldsymbol{\eta}_{t}(\gamma)$ is measurable and seperable.

(N-2). There is a $\gamma_{0} \in \Gamma$ such that $\boldsymbol{\lambda}_{T}\left(\gamma_{0}\right)=\mathbf{0}$.

(N-3). There are strictly positive numbers $a, b, c$ and $d_{0}$ such that for all $t$ and $n \geq n_{0}$

(i) $\left\|\boldsymbol{\lambda}_{T}(\gamma)\right\| \geq a\left\|\gamma-\gamma_{0}\right\|$ for $\left\|\gamma-\gamma_{0}\right\| \leq d_{0}$.

(ii) $\mathrm{E} \zeta_{t}(\gamma, d) \leq b d$ for $\left\|\gamma-\gamma_{0}\right\|+d \leq d_{0}$ and $d \geq 0$.

(iii) $\mathrm{E} \zeta_{t}^{2}(\gamma, d) \leq c d$ for $\left\|\gamma-\gamma_{0}\right\|+d \leq d_{0}$ and $d \geq 0$.

(N-4). $\mathrm{E}\left\|\boldsymbol{\eta}_{t}\left(\gamma_{0}\right)\right\|^{2}$ is finite.

Define $Z_{T}(\boldsymbol{\tau}, \boldsymbol{\gamma})=\frac{\left\|\sum_{t=1}^{T}\left[\boldsymbol{\eta}_{t}(\boldsymbol{\tau})-\boldsymbol{\eta}_{t}(\boldsymbol{\gamma})-\boldsymbol{\lambda}_{T}(\boldsymbol{\tau})+\boldsymbol{\lambda}_{T}(\boldsymbol{\gamma})\right]\right\|}{\sqrt{T}+T\left\|\boldsymbol{\lambda}_{T}(\boldsymbol{\tau})\right\|}$. Lemma 3.1 below is the essential ingredient in the proof of Equation (3.32). Pollard (1985) related this result to his concept of stochastic differentiability. The only difference between the results by Huber (1967) and Pollard (1985) is the region over which the supremum is computed. Pollard (1985) considers a sequence of balls that shrinks to $\gamma_{0}$ as $T$ grows, whereas the neighborhood of $\gamma_{0}$ is fixed in Huber's setting.

Lemma 3.1 Assumption $(N-1),(N-2)$ and $(N-3)$ imply $\sup _{\left\|\tau-\gamma_{0}\right\| \leq d_{0}} Z_{T}\left(\tau, \gamma_{0}\right) \stackrel{p}{\longrightarrow} 0$. 


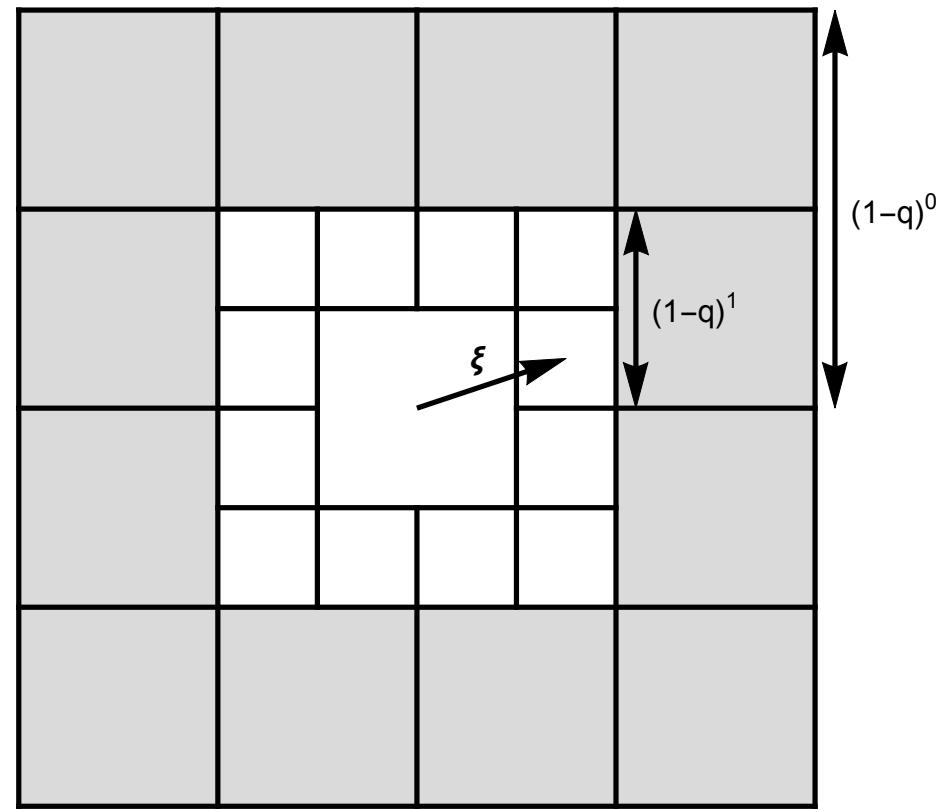

Figure 3.2: An overview of the geometry used in the proof by Huber (1967) for a $2 \mathrm{D}$ case. The set $\{\gamma \mid\|\gamma\| \leq 1\}$ is a square with left-bottom and right-top coordinates being $(-1,-1)$ and $(1,1)$, respectively. The graph shows the case $q=\frac{1}{2}$, and the gray area is $C_{0} \backslash C_{1}$ for this choice of $q$.

Proof All the norms in $\mathbb{R}^{M}$ are equivalent, so we may use the supremum norm without loss of generality. In this appendix all norms will refer to the supremum norm, i.e. for any vector $\gamma,\|\gamma\|=\max \left\{\left|\gamma_{1}\right|, \ldots,\left|\gamma_{M}\right|\right\}$. The coordinate system is chosen such that $\gamma_{0}=\mathbf{0}$ and $d_{0}=1$. With this choice of $\gamma$ and $d_{0}$ the supremum in the lemma is taken over $\|\tau\| \leq 1$

The approach by Huber (1967) subdivides the cube $\|\tau\| \leq 1$ into a large number of smaller cubes. One subsequently bounds $\operatorname{Pr}\left(Z_{n}(\tau, 0) \geq 2 \epsilon\right)$ on every cube. For an increasing number of cubes this probability declines more rapidly then the number of cubes increases, and uniform convergence follows.

Take $q=1 / M_{\varepsilon}$, with $M_{\varepsilon} \geq 2$ being an integer to be specified later. A sequence of concentric cubes $C_{k}$ is defined by

$$
C_{k}=\left\{\gamma \mid\|\gamma\| \leq(1-q)^{k}\right\}, \quad \text { for } k=0,1, \cdots, k_{0} .
$$


The difference $C_{k-1} \backslash C_{k}$ is divided into smaller cubes with edges of length $2 d=$ $(1-q)^{k-1} q$. The centers of these small cubes, $\boldsymbol{\xi}$, have a norm which satisfies $\|\boldsymbol{\xi}\|=$ $(1-q)^{k-1}\left(1-\frac{q}{2}\right)$. The situation is illustrated in Figure 3.2. The outer sides of the set $C_{k-1}$ have length $2(1-q)^{k-1}$, and hence there will fit $2(1-q)^{k-1} / 2 d=2 M_{\varepsilon}$ of these smaller cubes on an outer side. The set $C_{k-1}$ can be covered by $\left(2 M_{\varepsilon}\right)^{M}$ such small cubes, so for sure $C_{k-1} \backslash C_{k}$ can be covered by the same amount of small cubes. These small cubes will be use to cover the sets $C_{k-1} \backslash C_{k}$ for $k=1, \ldots, k_{0}$ and from the previous remark their total number $N$ is less than $k_{0}\left(2 M_{\varepsilon}\right)^{M}$. We number these small cubes $C_{(1)}, C_{(2)}, \ldots, C_{(N)}$. We have $\{\boldsymbol{\tau} \mid\|\boldsymbol{\tau}\| \leq 1\}=C_{k_{0}} \cup \bigcup_{j=1}^{N} C_{(j)}$ and hence, for any $\varepsilon>0$,

$$
\operatorname{Pr}\left(\sup _{\boldsymbol{\tau} \in C_{0}} Z_{T}(\boldsymbol{\tau}, \mathbf{0}) \geq 2 \varepsilon\right) \leq \operatorname{Pr}\left(\sup _{\boldsymbol{\tau} \in C_{k_{0}}} Z_{T}(\boldsymbol{\tau}, \mathbf{0}) \geq 2 \varepsilon\right)+\sum_{j=1}^{N} \operatorname{Pr}\left(\sup _{\boldsymbol{\tau} \in C_{(j)}} Z_{T}(\boldsymbol{\tau}, \mathbf{0}) \geq 2 \varepsilon\right) .
$$

It remains to specify the choice of $M_{\varepsilon}$ and $k_{0}=k_{0}(T)$. Huber (1967) uses

$$
M_{\varepsilon} \geq(3 b) /(\varepsilon a)
$$

and defines $k_{0}$ by

$$
(1-q)^{k_{0}} \leq T^{-\alpha} \leq(1-q)^{k_{0}-1}
$$

for an arbitrary fixed $\alpha$ in the range $\left(\frac{1}{2}, 1\right)$. From Equation (3.77) we have

$$
-k_{0}|\log (1-q)| \leq-\alpha \log (T) \leq-\left(k_{0}-1\right)|\log (1-q)| \Longrightarrow k_{0}(T)-1 \leq \frac{\alpha \log (T)}{|\log (1-q)|} \leq k_{0}(T),
$$

which shows $k_{0}=O(\log T)$. Since $\left(2 M_{\varepsilon}\right)^{M}$ does not depend on $T$, we also have $N=$ $O(\log T)$.

We first bound $\operatorname{Pr}\left(\sup _{\boldsymbol{\tau} \in C_{j}} Z_{n}(\boldsymbol{\tau}, \mathbf{0}) \geq 2 \varepsilon\right.$ ) for an arbitrary cube $C_{(j)}$ in $C_{k-1} \backslash C_{k}$. For $\tau \in C_{(j)}$ we have $\|\tau\| \geq(1-q)^{k}$. The edge length $d$ of such a cube satisfies $d=$ $\frac{1}{2}(1-q)^{k-1} q \leq(1-q)^{k} q$ because $\frac{1}{2} \leq(1-q)$. Assumption (N-3) gives

$$
\left\|\boldsymbol{\lambda}_{T}(\boldsymbol{\tau})\right\| \geq a\|\boldsymbol{\tau}\| \geq a(1-q)^{k}
$$


and

$$
\left\|\boldsymbol{\lambda}_{T}(\boldsymbol{\tau})-\boldsymbol{\lambda}_{T}(\boldsymbol{\xi})\right\| \leq \frac{1}{T} \sum_{t=1}^{T} \mathrm{E}\left\|\boldsymbol{\eta}_{t}(\boldsymbol{\gamma})-\boldsymbol{\eta}_{t}(\boldsymbol{\xi})\right\| \leq \frac{1}{T} \sum_{t=1}^{T} \mathrm{E} \zeta_{t}(\boldsymbol{\xi}, d) \leq b d \leq b q(1-q)^{k}
$$

Remark 3.7 Equation (3.80) illustrates why heterogeneity does not invalidate the approach by Huber (1967). The argument uses $\frac{1}{T} \sum_{t=1}^{T} \mathrm{E} \zeta_{t}(\boldsymbol{\xi}, d) \leq \frac{1}{T} \sum_{t=1}^{T} b d \leq b d$. The key observation is that Assumption $(N-3)$ is required to hold uniformly in $t$. The single terms as initially used in the i.i.d. setting of Huber (1967) can thus be replaced by their averaged counterparts by having a uniform bound on every element in the sum. This was first noted by Powell (1984).

In view of Assumption (N-2) we have for any $\tau$ in $C_{k-1} \backslash C_{k}$

$$
\begin{aligned}
Z_{T}(\boldsymbol{\tau}, \mathbf{0}) & =\frac{\left\|\sum_{t=1}^{T}\left[\boldsymbol{\eta}_{t}(\boldsymbol{\tau})-\boldsymbol{\eta}_{t}(\mathbf{0})-\boldsymbol{\lambda}_{T}(\tau)\right]\right\|}{\sqrt{T}+T\left\|\boldsymbol{\lambda}_{T}(\boldsymbol{\tau})\right\|} \\
& =\frac{\left\|\sum_{t=1}^{T}\left[\boldsymbol{\eta}_{t}(\boldsymbol{\tau})-\boldsymbol{\eta}_{t}(\boldsymbol{\xi})-\boldsymbol{\lambda}_{T}(\boldsymbol{\tau})+\boldsymbol{\lambda}_{T}(\boldsymbol{\xi})+\boldsymbol{\eta}_{t}(\boldsymbol{\xi})-\boldsymbol{\eta}_{t}(\mathbf{0})-\boldsymbol{\lambda}_{t}(\boldsymbol{\xi})\right]\right\|}{\sqrt{T}+T\left\|\boldsymbol{\lambda}_{T}(\boldsymbol{\tau})\right\|} \\
& \leq \frac{\left\|\sum_{t=1}^{T}\left[\boldsymbol{\eta}_{t}(\boldsymbol{\tau})-\boldsymbol{\eta}_{t}(\boldsymbol{\xi})-\boldsymbol{\lambda}_{T}(\boldsymbol{\tau})+\boldsymbol{\lambda}_{T}(\boldsymbol{\xi})\right]\right\|}{T a(1-q)^{k}}+\frac{\left\|\sum_{t=1}^{T}\left[\boldsymbol{\eta}_{t}(\boldsymbol{\xi})-\boldsymbol{\eta}_{t}(\mathbf{0})-\boldsymbol{\lambda}_{t}(\boldsymbol{\xi})\right]\right\|}{T a(1-q)^{k}} \\
& \leq \frac{\sum_{t=1}^{T}\left[\left\|\boldsymbol{\eta}_{t}(\boldsymbol{\tau})-\boldsymbol{\eta}_{t}(\boldsymbol{\xi})\right\|+\left\|\boldsymbol{\lambda}_{T}(\boldsymbol{\tau})-\boldsymbol{\lambda}_{T}(\boldsymbol{\xi})\right\|\right]}{T a(1-q)^{k}}+\frac{\left\|\sum_{t=1}^{T}\left[\boldsymbol{\eta}_{t}(\boldsymbol{\xi})-\boldsymbol{\eta}_{t}(\mathbf{0})-\boldsymbol{\lambda}_{t}(\boldsymbol{\xi})\right]\right\|}{T a(1-q)^{k}} \\
& \leq \frac{\sum_{t=1}^{T}\left[\zeta_{t}(\boldsymbol{\xi}, d)+\mathrm{E} \zeta_{t}(\boldsymbol{\xi}, d)\right]}{T a(1-q)^{k}}+\frac{\left\|\sum_{t=1}^{T}\left[\boldsymbol{\eta}_{t}(\boldsymbol{\xi})-\boldsymbol{\eta}_{t}(\mathbf{0})-\boldsymbol{\lambda}_{t}(\boldsymbol{\xi})\right]\right\|}{T a(1-q)^{k}}:=U_{T}+V_{T}
\end{aligned}
$$

Moreover, Equation (3.80) and Equation (3.76) imply $\varepsilon a \geq 3 b q$, hence

$$
\begin{aligned}
\operatorname{Pr}\left(U_{T} \geq \varepsilon\right) & =\operatorname{Pr}\left(\sum_{t=1}^{T}\left[\zeta_{t}(\boldsymbol{\xi}, d)-\mathrm{E} \zeta_{t}(\boldsymbol{\xi}, d)\right] \geq T \varepsilon a(1-q)^{k}-2 \sum_{t=1}^{T} \mathrm{E} \zeta_{t}(\boldsymbol{\xi}, d)\right) \\
& \leq \operatorname{Pr}\left(\sum_{t=1}^{T}\left[\zeta_{t}(\boldsymbol{\xi}, d)-\mathrm{E} \zeta_{t}(\boldsymbol{\xi}, d)\right] \geq T \varepsilon a(1-q)^{k}-2 T b q(1-q)^{k}\right) \\
& \leq \operatorname{Pr}\left(\sum_{t=1}^{T}\left[\zeta_{t}(\boldsymbol{\xi}, d)-\mathrm{E} \zeta_{t}(\boldsymbol{\xi}, d)\right] \geq T b q(1-q)^{k}\right) .
\end{aligned}
$$

Assumption $(N-3)$ makes sure that the variance contribution is linear in the sample size. Remark 3.8 provides the details on this point. The probability in Equation (3.82) can be 
bounded above using Chebyshev's inequality, that is

$$
\operatorname{Pr}\left(U_{T} \geq \varepsilon\right) \leq \frac{c d}{T b^{2} q^{2}(1-q)^{2 k}} \leq \frac{c}{b^{2} q(1-q)} \frac{1}{T(1-q)^{k-1}} \leq \frac{c}{b^{2} q(1-q)} T^{\alpha-1}
$$

An upper bound on $\operatorname{Pr}\left(V_{T} \geq \varepsilon\right)$ is obtained along the same lines. The only difference is that $V_{T}$ is already a sum with a mean of zero, which makes the bound sharper.

$$
\begin{aligned}
\operatorname{Pr}\left(V_{T} \geq \varepsilon\right) & \leq \operatorname{Pr}\left(\sum_{t=1}^{T}\left[\boldsymbol{\eta}_{t}(\boldsymbol{\xi})-\boldsymbol{\eta}_{t}(\mathbf{0})-\boldsymbol{\lambda}_{T}(\boldsymbol{\xi})\right] \geq 3 b q T(1-q)^{k}\right) \\
& \leq \frac{c\|\boldsymbol{\xi}\|}{9 b^{2} q^{2} T(1-q)^{2 k}} \leq \frac{c}{9 b^{2} q^{2}(1-q)^{2}} T^{\alpha-1}
\end{aligned}
$$

The results in Equations 3.83 and 3.84 combine to $\operatorname{Pr}\left(\sup _{\tau \in C_{(j)}} Z_{T}(\tau, 0) \geq 2 \varepsilon\right) \leq \operatorname{Pr}\left(U_{T} \geq \varepsilon\right)+$ $\operatorname{Pr}\left(V_{T} \geq \varepsilon\right) \leq K \times T^{\alpha-1}$, with the multiplicative constant given by $K=\frac{c}{b^{2} q(1-q)}+\frac{c}{9 b^{2} q^{2}(1-q)^{2}}$. This bound is valid for any cube $C_{(j)}$. The total number of cubes was of the order $O(\log (T))$. The second term of Equation 3.75 therefore satisfies

$$
\sum_{j=1}^{N} \operatorname{Pr}\left(\sup _{\boldsymbol{\tau} \in C_{(j)}} Z_{T}(\boldsymbol{\tau}, \mathbf{0}) \geq 2 \varepsilon\right) \leq N \times K \times T^{\alpha-1}=O\left(T^{\alpha-1} \log (T)\right) .
$$

The choice of $k_{0}$ guarantees that $\alpha-1 \in(-1 / 2,0)$ and the supremum of over $C_{0} \backslash C_{k_{0}}$ goes to zero as $T$ becomes large.

It remains to derive a uniform bound over the cube which is centered at the origin. The distance from any point in $C_{k_{0}}$ to the origin is at most $d=(1-q)^{k_{0}} \leq T^{-\alpha}$. Contrary to Equation (3.81), we now use the upper bound

$$
Z_{T}(\boldsymbol{\tau}, \mathbf{0}) \leq \frac{1}{\sqrt{T}} \sum_{t=1}^{T}\left[\left\|\boldsymbol{\eta}_{t}(\boldsymbol{\tau})-\boldsymbol{\eta}_{t}(\mathbf{0})\right\|+\left\|\boldsymbol{\lambda}_{T}(\boldsymbol{\tau})\right\|\right] \leq \frac{1}{\sqrt{T}} \sum_{t=1}^{T}\left[\zeta_{t}(\mathbf{0}, d)+\mathrm{E} \zeta_{t}(\mathbf{0}, d)\right],
$$

for all $\tau$ in $C_{k_{0}} \cdot \sum_{t=1}^{T} \mathrm{E} \zeta_{t}(\mathbf{0}, d) \leq b d T \leq b T^{1-\alpha}$ is growing at a slower rate than $T^{1 / 2}$. We can thus find a $T_{0}$ such that $2 \sqrt{T} \varepsilon-2 \sum_{t=1}^{T} \mathrm{E} \zeta_{t}(\mathbf{0}, d) \geq \sqrt{T} \varepsilon$ holds for all $T \geq T_{0}$. For $T \geq T_{0}$, we apply Chebyshev's inequality to find

$$
\begin{aligned}
\operatorname{Pr}\left(\sup _{\boldsymbol{\tau} \in C_{k_{0}}} Z_{T}(\boldsymbol{\tau}, \mathbf{0}) \geq 2 \varepsilon\right) & \leq \operatorname{Pr}\left(\sum_{t=1}^{T}\left[\zeta_{t}(\mathbf{0}, d)-\mathrm{E} \zeta_{t}(\mathbf{0}, d)\right] \geq 2 \sqrt{T} \varepsilon-2 \sum_{t=1}^{T} \mathrm{E} \zeta_{t}(\mathbf{0}, d)\right) \\
& \leq \operatorname{Pr}\left(\sum_{t=1}^{T}\left[\zeta_{t}(\mathbf{0}, d)-\mathrm{E} \zeta_{t}(\mathbf{0}, d)\right] \geq \sqrt{T} \varepsilon\right) \leq c \varepsilon^{-2} T^{-\alpha}
\end{aligned}
$$


The proof of Lemma 3.1 follows from Equation (3.75), (3.85) and (3.87).

Remark 3.8 To apply the Chebyshev inequalities as used in Equation (3.83), (3.84) and (3.87), we need to bound $\left.\operatorname{var}\left(\sum_{t=1}^{T}\left(\zeta_{t}(\boldsymbol{\xi}, d)\right)-\mathrm{E} \zeta_{t}(\boldsymbol{\xi}, d)\right)\right)$. For independent observations such as considered by Huber (1967) and Powell (1984) this is straightforward because Assumption (N-3)(iii) provides

$$
\left.\operatorname{var}\left(\sum_{t=1}^{T}\left(\zeta_{t}(\boldsymbol{\xi}, d)\right)-\mathrm{E} \zeta_{t}(\boldsymbol{\xi}, d)\right)\right)=\sum_{t=1}^{T} \operatorname{var}\left(\zeta_{t}(\boldsymbol{\xi}, d)\right) \leq \sum \mathrm{E} \zeta_{t}^{2}(\boldsymbol{\xi}, d) \leq c d T
$$

We can derive a similar bound if $\zeta_{t}(\gamma, d)$ is an $L_{2}$-mixingale with $c_{t}=\sup \left\|\zeta_{t}(\gamma, d)\right\|_{2}$ and summable $L_{2}$-mixingale numbers $\left\{\psi_{m}\right\}$. In view of the discussion on page 467 of Andrews (1988) and (N-3) (iii) we have

$$
\left.\operatorname{var}\left(\sum_{t=1}^{T}\left(\zeta_{t}(\boldsymbol{\xi}, d)\right)-\mathrm{E} \zeta_{t}(\boldsymbol{\xi}, d)\right)\right) \leq 8 T \sup _{t} \mathrm{E} \zeta_{t}^{2}(\boldsymbol{\xi}, d) \sum_{m=1}^{\lfloor T / 2\rfloor} \psi_{m} \leq 8 c d T \sum_{m=1}^{\lfloor T / 2\rfloor} \psi_{m}
$$

where $\lfloor x\rfloor$ denotes the integer part of $x$. 


\section{C Supplementary Simulation Results}

\section{C.1 Histograms for Larger Sample Sizes}

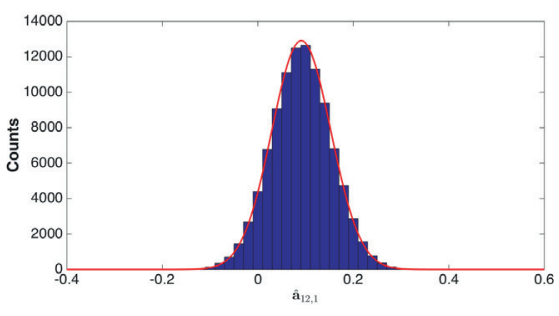

(a) Constrained

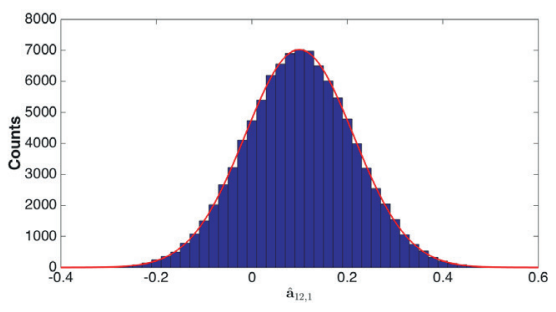

(b) Unconstrained

Figure 3.3: Identical to Figure 3.1, but for $T=250$.

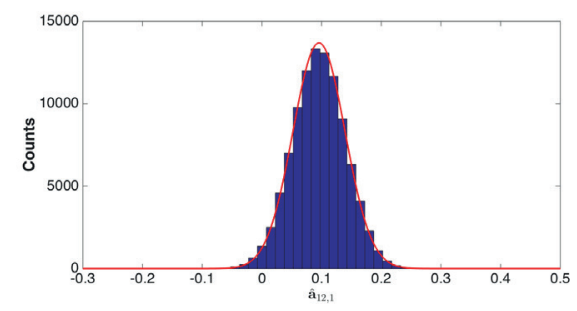

(a) Constrained

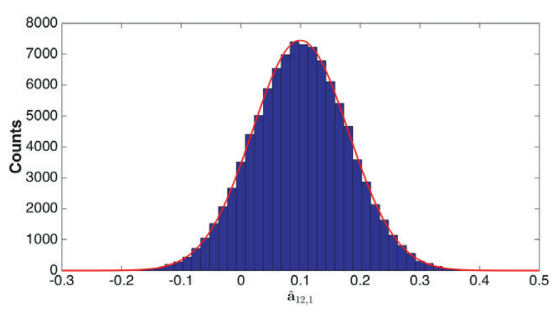

(b) Unconstrained

Figure 3.4: Identical to Figure 3.1, but for $T=500$. 


\section{C.2 Information Criteria for Larger Sample Sizes}

\begin{tabular}{cc||c|c|c|c} 
& $\mathrm{p}$ & 0 & 1 & 2 & 3 \\
\hline \hline \multirow{2}{*}{$\mathcal{N}(0,1)$} & LAD & 0.00 & 98.01 & 1.84 & 0.15 \\
& OLS & 0.00 & 99.86 & 0.14 & 0.00 \\
\hline \multirow{2}{*}{ Laplace } & LAD & 0.00 & 99.71 & 0.29 & 0.00 \\
& OLS & 0.00 & 99.88 & 0.11 & 0.01 \\
\hline \multirow{2}{*}{$\mathrm{t}(3)$} & LAD & 0.00 & 99.36 & 0.60 & 0.04 \\
& OLS & 0.01 & 99.73 & 0.26 & 0.00 \\
\hline \multirow{2}{*}{$\mathrm{t}(4)$} & LAD & 0.00 & 99.09 & 0.91 & 0.00 \\
& OLS & 0.00 & 99.80 & 0.20 & 0.00 \\
\hline \multirow{2}{*}{$\mathrm{t}(5)$} & LAD & 0.00 & 99.11 & 0.85 & 0.04 \\
& OLS & 0.00 & 99.84 & 0.15 & 0.01 \\
\hline \multirow{2}{*}{$\mathrm{t}(10)$} & LAD & 0.00 & 98.67 & 1.26 & 0.07 \\
& OLS & 0.00 & 99.92 & 0.08 & 0.00 \\
\hline \multirow{2}{*}{$\mathrm{t}(20)$} & LAD & 0.00 & 98.56 & 1.37 & 0.07 \\
& OLS & 0.00 & 99.89 & 0.10 & 0.01
\end{tabular}

Table 3.15: Identical to Table 3.13, but for $T=100$.

\begin{tabular}{lc||c|c|c|c} 
& $\mathrm{p}$ & 0 & 1 & 2 & 3 \\
\hline \hline \multirow{2}{*}{$\varepsilon=0.01$} & LAD & 0.01 & 98.07 & 1.80 & 0.12 \\
& OLS & 0.01 & 99.82 & 0.17 & 0.00 \\
\hline \multirow{2}{*}{$\varepsilon=0.02$} & LAD & 0.00 & 98.44 & 1.48 & 0.08 \\
& OLS & 0.00 & 99.79 & 0.20 & 0.01 \\
\hline \multirow{2}{*}{$\varepsilon=0.03$} & LAD & 0.00 & 98.65 & 1.29 & 0.06 \\
& OLS & 0.00 & 99.78 & 0.20 & 0.02 \\
\hline \multirow{2}{*}{$\varepsilon=0.04$} & LAD & 0.00 & 98.68 & 1.26 & 0.06 \\
& OLS & 0.00 & 99.81 & 0.17 & 0.02 \\
\hline \multirow{2}{*}{$\varepsilon=0.05$} & LAD & 0.00 & 98.77 & 1.16 & 0.07 \\
& OLS & 0.00 & 99.79 & 0.20 & 0.01 \\
\hline \multirow{2}{*}{$\varepsilon=0.06$} & LAD & 0.00 & 98.88 & 1.07 & 0.05 \\
& OLS & 0.00 & 99.75 & 0.24 & 0.01 \\
\hline \multirow{2}{*}{$\varepsilon=0.07$} & LAD & 0.00 & 98.90 & 1.06 & 0.04 \\
& OLS & 0.00 & 99.73 & 0.25 & 0.02 \\
\hline \multirow{2}{*}{$\varepsilon=0.08$} & LAD & 0.00 & 99.13 & 0.85 & 0.02 \\
& OLS & 0.00 & 99.73 & 0.25 & 0.02 \\
\hline \multirow{2}{*}{$\varepsilon=0.09$} & LAD & 0.00 & 99.19 & 0.80 & 0.01 \\
& OLS & 0.00 & 99.73 & 0.26 & 0.01 \\
\hline \multirow{2}{*}{$\varepsilon=0.10$} & LAD & 0.00 & 99.40 & 0.59 & 0.01 \\
& OLS & 0.00 & 99.72 & 0.26 & 0.02
\end{tabular}

Table 3.16: Identical to Table 3.14, but for $T=100$. 


\begin{tabular}{cc||c|c|c|c} 
& $\mathrm{p}$ & 0 & 1 & 2 & 3 \\
\hline \hline \multirow{2}{*}{$\mathcal{N}(0,1)$} & LAD & 0.00 & 99.01 & 0.96 & 0.03 \\
& OLS & 0.00 & 99.91 & 0.09 & 0.00 \\
\hline \multirow{2}{*}{ Laplace } & LAD & 0.00 & 99.82 & 0.18 & 0.00 \\
& OLS & 0.00 & 99.90 & 0.10 & 0.00 \\
\hline \multirow{2}{*}{$\mathrm{t}(3)$} & LAD & 0.00 & 99.67 & 0.32 & 0.01 \\
& OLS & 0.00 & 99.81 & 0.18 & 0.01 \\
\hline \multirow{2}{*}{$\mathrm{t}(4)$} & LAD & 0.00 & 99.62 & 0.36 & 0.02 \\
& OLS & 0.00 & 99.92 & 0.08 & 0.00 \\
\hline \multirow{2}{*}{$\mathrm{t}(5)$} & LAD & 0.00 & 99.46 & 0.51 & 0.03 \\
& OLS & 0.00 & 99.89 & 0.11 & 0.00 \\
\hline \multirow{2}{*}{$\mathrm{t}(10)$} & LAD & 0.00 & 99.23 & 0.74 & 0.03 \\
& OLS & 0.00 & 99.91 & 0.09 & 0.00 \\
\hline \multirow{2}{*}{$\mathrm{t}(20)$} & LAD & 0.00 & 99.05 & 0.89 & 0.06 \\
& OLS & 0.00 & 99.95 & 0.05 & 0.00
\end{tabular}

Table 3.17: Identical to Table 3.13, but for $T=150$.

\begin{tabular}{lc||c|c|c|c} 
& $\mathrm{p}$ & 0 & 1 & 2 & 3 \\
\hline \hline \multirow{2}{*}{$\varepsilon=0.01$} & LAD & 0.00 & 99.02 & 0.94 & 0.04 \\
& OLS & 0.00 & 99.96 & 0.04 & 0.00 \\
\hline \multirow{2}{*}{$\varepsilon=0.02$} & LAD & 0.00 & 99.11 & 0.87 & 0.02 \\
& OLS & 0.00 & 99.85 & 0.15 & 0.00 \\
\hline \multirow{2}{*}{$\varepsilon=0.03$} & LAD & 0.00 & 99.14 & 0.85 & 0.01 \\
& OLS & 0.00 & 99.86 & 0.14 & 0.00 \\
\hline \multirow{2}{*}{$\varepsilon=0.04$} & LAD & 0.00 & 99.22 & 0.76 & 0.02 \\
& OLS & 0.00 & 99.92 & 0.08 & 0.00 \\
\hline \multirow{2}{*}{$\varepsilon=0.05$} & LAD & 0.00 & 99.37 & 0.60 & 0.03 \\
& OLS & 0.00 & 99.89 & 0.11 & 0.00 \\
\hline \multirow{2}{*}{$\varepsilon=0.06$} & LAD & 0.00 & 99.33 & 0.66 & 0.01 \\
& OLS & 0.00 & 99.91 & 0.09 & 0.00 \\
\hline \multirow{2}{*}{$\varepsilon=0.07$} & LAD & 0.00 & 99.39 & 0.59 & 0.02 \\
& OLS & 0.00 & 99.91 & 0.09 & 0.00 \\
\hline \multirow{2}{*}{$\varepsilon=0.08$} & LAD & 0.00 & 99.37 & 0.60 & 0.03 \\
& OLS & 0.00 & 99.92 & 0.07 & 0.01 \\
\hline \multirow{2}{*}{$\varepsilon=0.09$} & LAD & 0.00 & 99.53 & 0.47 & 0.00 \\
& OLS & 0.00 & 99.89 & 0.11 & 0.00 \\
\hline \multirow{2}{*}{$\varepsilon=0.10$} & LAD & 0.00 & 99.63 & 0.37 & 0.00 \\
& OLS & 0.00 & 99.90 & 0.10 & 0.00
\end{tabular}

Table 3.18: Identical to Table 3.14, but for $T=150$. 


\section{Chapter 4}

\section{Residual Bootstrap for VAR}

\section{Models Estimated by Least}

\section{Absolute Deviations}

"The new methods are fantastic computational spendthrifts by the standards of the past. As we shall see they can easily expend a million arithmetic operations on the analysis of

fifteen data points. The payoff for all this computation is freedom from two limiting factors which have dominated statistical theory since Gauss' time (1800), the bell-shaped curve and linear mathematics. We will focus on one of these methods, the bootstrap."

- Diaconis and Efron (1983) 


\section{Abstract}

We consider the fixed design residual bootstrap method to conduct inference in stationary vector autoregressive models. This bootstrap method keeps the lagged dependent variables equal to their sample realizations while resampling. The asymptotic validity of this method is established. We also show that bootstrapped Wald and LM type of test statistics can be used to test linear hypotheses. Our method does not rely on density estimation and is thus easy to apply. A Monte Carlo study reports on the finite sample performance. This simulation study suggests that the fixed design bootstrap has good size properties and better size properties than a recursive bootstrap scheme. The bootstrapped version of the Wald type of test has a higher power than both the bootstrapped version of the LM test and the asymptotically pivotal tests that require density estimation. The bootstrap Wald type of test is therefore recommended to practitioners. 


\subsection{Introduction}

Inference by least absolute deviation (LAD) is less popular than its least squares (LS) counterpart. This is rather surprising given the superior performance of LAD estimation in the presence of fat tailed innovations and/or outliers. Three possible explanations for this lack of popularity are: (1) the absence of a closed-form solution for the LAD estimator making it difficult to derive its properties; (2) it took some time before a solution algorithm for the LAD estimator became available during which the LS estimator gained great popularity (see chapter 1 of Bloomfield and Steiger (1983) for the historical developments of LAD and quantile estimation algorithms); and (3) statistical inference is hampered by the dependency of the asymptotic covariance matrix of the LAD estimator on the density of the innovation distribution. The first and second reasons are closely related but they are of theoretical and practical relevance, respectively. We now elaborate on these three points.

The asymptotic properties of the LAD/quantile estimators in univariate models have been studied in detail by various authors such as Koenker and Bassett (1978), Koenker and Bassett (1982), Knight (1988), Phillips (1991) and Weiss (1991). A reference to models with multiple equations is White et al. (2015).

Harris (1950) showed how the least absolute deviations estimator can be computed from a linear program. Concrete solution algorithms were proposed by Barrodale and Roberts (1978), Bartels et al. (1978) and Bloomfield and Steiger (1980). All three papers exploit the fact that at least $r$ residuals vanish for an $r$-dimensional parameter vector. For $n$ data points this leaves $\left(\begin{array}{l}n \\ r\end{array}\right)$ possible combinations of having $r$ zero residuals. The algorithms in the aforementioned references differ only in the rules that are used to decide on the leaving and entering zero residual.

The asymptotic covariance matrix of the LAD estimator requires the estimation of the density of the innovation at zero. One of the earliest references is Buchinsky (1995), who explored various methods by means of a Monte Carlo study. The data generating process of his Monte Carlo study originated from an estimated model on survey data. In this study, confidence intervals constructed by either a bootstrap method or an order statistics method provided empirical coverage closest to the nominal level. The recent literature on $\mathrm{LAD}$ /quantile estimation in time series modeling makes use of: order statistics (Koenker and Xiao (2006)), bootstrap resampling (Xiao (2009)), and kernel density estimation (Engle and Manganelli (2004), Cho et al. (2015) and White et al. (2015)). 
Bootstrap methods for autoregressive (AR) processes have been extensively studied in the context of least squares estimation. Kreiss (1997) discusses the bootstrap consistency of the residual bootstrap, the Wild bootstrap and spectral density estimators. Around the same time, Paparoditis (1996) used a recursive residual bootstrap to bootstrap the MA parameters of an infinite order autoregressive process. Two additional reference on AR processes of infinite order are Inoue and Kilian (2002) and Gonçalves and Kilian (2007). The first reference generalizes the bootstrap validity to functional transformations of the LS coefficients whereas the second reference relaxes the i.i.d. assumption on the innovations by allowing for conditional heteroskedasticity. Brüggemann et al. (2016) have pointed out that the fixed-design Wild bootstrap discussed in Gonçalves and Kilian (2007) cannot always replicate the distribution of the variance parameters (as relevant for impulse response analysis). They propose a residual-based moving block bootstrap to secure asymptotically valid inference when both the autoregressive and variance parameters are of interest.

The previous references consider least squares estimation. References on other estimation methods are Lahiri (1992) on M-estimators within an i.i.d. regression framework and Gonçalves and White (2004) on maximum likelihood estimation of nonlinear dynamic models. Dedicated references on LAD/quantile estimation are: Hahn (1995), Fitzenberger (1997), Horowitz (1998) and Feng et al. (2011). Hahn (1995) considers a linear regression model and proves the validity of the residual bootstrap for fixed regressors (assuming i.i.d. unobserved errors) and the pairs bootstrap for random regressors (assuming the explanatory variables and errors to form an i.i.d. sequence). Dependency is allowed in the linear regression setting studied by Fitzenberger (1997) who establishes consistency of the moving block bootstrap while assuming strong mixing conditions on the explanatory variables and innovations. The approach by Horowitz (1998) is different in the sense that he approximates the absolute value in the LAD objective function by a sequence of continuous functions. These continuous functions are indexed by a parameter that controls the curvature at zero. This parameter shrinks to zero rapidly enough for the smoothed and normal LAD estimator to have the same limiting distribution. A pairs bootstrap method is shown to be asymptotically valid. The Wild bootstrap of Feng et al. (2011) allows the errors of the linear regression to be heteroskedastic.

In this chapter we consider a residual bootstrap procedure to conduct inference on the coefficients of vector autoregressive models as estimated by least absolute deviations. We show that the asymptotic distribution of the bootstrap estimator coincides with the large sample Gaussian approximation. In other words, our bootstrap proposal is asymptotically valid. We also consider bootstrap versions of two test statistics and show that 
their asymptotic distributions are correctly replicated in the bootstrap. These bootstrap procedures circumvent the need for density estimation and should therefore make the estimation of VAR models by LAD more accessible to applied researchers. The simulation study shows that the Wald type bootstrap test has higher empirical power.

\subsection{Theory}

\subsubsection{The Model}

The model setting is similar to the setting from Chapter 3. That is, we study a $K$ dimensional vector autoregression of order $p$ given by

$$
\boldsymbol{y}_{t}=\boldsymbol{\nu}+\boldsymbol{A}_{1} \boldsymbol{y}_{t-1}+\cdots+\boldsymbol{A}_{p} \boldsymbol{y}_{t-p}+\boldsymbol{u}_{t}
$$

with intercept vector $\boldsymbol{\nu}$ and $(K \times K)$ coefficient matrices $\boldsymbol{A}_{i}(i=1,2, \ldots, p)$. As in Lütkepohl (2005), we define

$$
\begin{aligned}
& \boldsymbol{Y}:=\left(\boldsymbol{y}_{1}, \ldots, \boldsymbol{y}_{T}\right) \quad(K \times T), \\
& \boldsymbol{B}:=\left(\boldsymbol{\nu}, \boldsymbol{A}_{1}, \ldots, \boldsymbol{A}_{p}\right) \quad(K \times(K p+1)), \\
& z_{t}:=\left(1, \boldsymbol{y}_{t}^{\prime}, \ldots, \boldsymbol{y}_{t-p+1}^{\prime}\right)^{\prime} \quad((K p+1) \times 1), \\
& Z:=\left(z_{0}, \ldots, z_{T-1}\right) \quad((K p+1) \times T), \\
& \boldsymbol{U}:=\left(\boldsymbol{u}_{1}, \ldots, \boldsymbol{u}_{T}\right) \quad(K \times T) .
\end{aligned}
$$

These definitions allow us to express Equation (4.1) as $\boldsymbol{y}_{t}=\boldsymbol{B} \boldsymbol{z}_{t}+\boldsymbol{u}_{t}$ and the column stack over all $T$ observations as $\boldsymbol{Y}=\boldsymbol{B} \boldsymbol{Z}+\boldsymbol{U}$. All $K(K p+1)$ parameters are collected in the vector $\boldsymbol{b}=\operatorname{vec}\left(\boldsymbol{B}^{\prime}\right)$. This vector can be subjected to linear constraints such that $\boldsymbol{b}=\boldsymbol{R} \gamma+\boldsymbol{r}$ holds for some known $(K(K p+1) \times M)$ matrix $\boldsymbol{R}$ and some known $K(K p+1)$ vector $\boldsymbol{r}$. The parameter vector $\gamma$ of length $M$ is supposed to be an element of the finite dimensional parameter space $\Gamma$. The true parameter vector is $\gamma_{0}$ (which implies $\boldsymbol{b}_{0}=$ $\left.\boldsymbol{R} \gamma_{0}+\boldsymbol{r}\right)$. We denote by $\boldsymbol{R}_{k}$ and $\boldsymbol{r}_{k}$ the parts of respectively $\boldsymbol{R}$ and $\boldsymbol{r}$ that are related to the parameters in the $k$ 'th cross-section. The LAD estimator for the parameter vector $\gamma_{0}$ is defined as

$$
\widehat{\gamma}_{T}=\arg \min _{\boldsymbol{\gamma} \in \Gamma} \frac{1}{T} \sum_{t=1}^{T} \sum_{k=1}^{K}\left|y_{k t}-\boldsymbol{z}_{t-1}^{\prime}\left(\boldsymbol{R}_{k} \gamma+\boldsymbol{r}_{k}\right)\right|:=\arg \min _{\boldsymbol{\gamma} \in \Gamma} S_{T}(\boldsymbol{\gamma}) .
$$


This objective function sums the absolute value of the residuals instead of the well-known sum of squared residuals from the LS approach. The merits of LAD estimation for VAR models have been discussed in Chapter 3. Our theoretical results are derived under the following set of assumptions.

Assumption 4.1 (Stability) Consider the set of matrices $\left\{\boldsymbol{A}_{1}, \ldots, \boldsymbol{A}_{p}\right\}$ implied by the definition in Equation (4.1) and $\boldsymbol{b}=\boldsymbol{R} \boldsymbol{\gamma}+\boldsymbol{r}$. For all $\boldsymbol{\gamma} \in \Gamma, \operatorname{det}(\boldsymbol{A}(z))=\operatorname{det}\left(\boldsymbol{I}_{K} z^{p}-\right.$ $\left.\boldsymbol{A}_{1} z^{p-1}-\ldots-\boldsymbol{A}_{p}\right) \neq 0$ for all $|z| \geq 1$.

Assumption 4.2 (InNovations) The innovation process $\left\{\boldsymbol{u}_{t}\right\}$ satisfies the conditions:

(a) $\left\{\boldsymbol{u}_{t}\right\}$ is independent and identically distributed (i.i.d.).

(b) $\mathrm{E}\left\|\boldsymbol{u}_{t}\right\|^{2+\delta}<\infty$ for some $\delta>0$.

We denote the k'th component of $\boldsymbol{u}_{t}$ by $u_{k t}$, then for every $k=1,2, \ldots, K$ :

(c) $u_{k t}$ has median zero and admits a density $f_{k}$ that is bounded away from zero, i.e. $\min _{k=1,2, \ldots, K} f_{k}(x)>0$ for a neighborhood around $x=0$.

(d) The density $f_{k}$ is bounded, i.e. $\sup _{x} \max _{k=1,2, \ldots, K} f_{k}(x)<\infty$.

(e) $f_{k}$ is Lipschitz continuous such that there exists a positive constant $L_{0}$ such that $\left|f_{k}\left(x_{1}\right)-f_{k}\left(x_{2}\right)\right| \leq L_{0}\left|x_{1}-x_{2}\right|$ for all $x_{1}$ and $x_{2}$.

Assumption 4.3 (CompaCTNess) $\Gamma$ is a compact subset of $\mathbb{R}^{M}$.

Assumption 4.4 (Positive Definiteness) The following matrices are positive definite: (a) $\boldsymbol{\Lambda}=\mathrm{E}\left(\boldsymbol{z}_{t-1} \boldsymbol{z}_{t-1}^{\prime}\right)$ and $(b) \mathrm{E}(\boldsymbol{\Psi})$ with $(i, j)^{\prime}$ th element $\mathrm{E}\left(\boldsymbol{\Psi}_{i j}\right)=\mathrm{E}\left(\operatorname{sgn}\left(u_{i t}\right) \operatorname{sgn}\left(u_{j t}\right)\right)$.

Assumption 4.5 (No Parameter at the Boundary) $\gamma_{0}$ is interior to $\Gamma$.

Assumption 4.1 rules out both unit roots and explosive behavior. In combination with Assumption 4.2(a)-(b), this implies that $\left\{\boldsymbol{y}_{t}\right\}$ is both weakly and strictly stationary. More technical assumptions on the innovations are stated in Assumption 4.2(c)-(e). Compactness of the parameter space makes the estimator measurable and facilitates the extension of point wise convergence of the objective function to uniform convergence. The positive definiteness of the matrices in Assumption 4.4 is needed for parameter identification and a non-degenerate limiting distribution. The current set of assumptions is stronger than the 
assumptions we encountered in Chapter 3. We can thus invoke the results from Chapter 3 to immediately obtain the following theorem.

\section{Theorem 4.1 (Asymptotic Properties of the LAD estimator)}

(a) Under Assumptions 4.1, 4.2(a)-(c), 4.3 and 4.4(a), we have $\widehat{\gamma}_{T} \rightarrow \gamma_{0}$ almost surely.

(b) Suppose that Assumptions 4.1-4.5 hold. Then,

$$
\begin{aligned}
& \qquad \sqrt{T}\left(\widehat{\gamma}_{T}-\gamma_{0}\right) \stackrel{d}{\longrightarrow} \mathrm{N}\left(\mathbf{0}, \frac{1}{4}\left[\boldsymbol{R}^{\prime}(\boldsymbol{\Xi} \otimes \boldsymbol{\Lambda}) \boldsymbol{R}\right]^{-1} \boldsymbol{R}^{\prime}(\mathrm{E}(\boldsymbol{\Psi}) \otimes \boldsymbol{\Lambda}) \boldsymbol{R}\left[\boldsymbol{R}^{\prime}(\boldsymbol{\Xi} \otimes \boldsymbol{\Lambda}) \boldsymbol{R}\right]^{-1}\right), \\
& \text { with } \boldsymbol{\Xi}=\operatorname{diag}\left(f_{1}(0), f_{2}(0), \ldots, f_{K}(0)\right) \text {. }
\end{aligned}
$$

Theorem 4.1 shows that the limiting distribution of $\sqrt{T}\left(\widehat{\gamma}_{T}-\gamma_{0}\right)$ depends on the density of the (marginal) innovation distributions evaluated at zero. In Chapter 3, we used a kernel density estimator to obtain consistent estimates of these densities. The performance of these density estimates depends on the selected bandwidth and a wellperforming bandwidth selection method can be hard to find. ${ }^{1}$ In the next section, we will introduce a bootstrap method to directly replicate the asymptotic distribution of various test statistics. The density estimation step is thereby avoided.

Remark 4.1 Equation (4.1) defines a VAR of finite lag order. Paparoditis (1996), Inoue and Kilian (2002) and Gonçalves and Kilian (2007) discuss bootstrap routines for autoregressions of infinite order. All three references rely on least squares estimation and therefore solely exploit the information in the second moments of the time series. Their truncation errors are bounded using results on the spectral properties of infinite order autoregressive models (see Berk (1974) and Lewis and Reinsel (1985)). These results do not easily carry over to our setting because the LAD estimator does not only depend on second order properties. Generalizations of our results to infinite order autoregressions are left for future research.

\subsubsection{The Bootstrap}

We will study the following bootstrap method: the residual bootstrap with fixed design. In this section we shall establish the validity of this bootstrap proposal when applied to LAD estimation in VAR models.

\footnotetext{
${ }^{1}$ This point is perhaps best illustrated by the fact that there exists a voluminous literature on bandwidth selection. Some references are: Hall et al. (1991), Jones et al. (1996) and Botev et al. (2010).
} 
The intuition behind residual bootstrap methods is as follows. Based on the observed sample one generates new datasets with distributional properties that approximate the true DGP. For residual bootstrap methods, such an approximation can be motivated from the following equality concerning the residuals: $\widehat{u}_{k t}=u_{k t}-z_{t-1}^{\prime} \boldsymbol{R}_{k}\left(\widehat{\gamma}_{T}-\gamma_{0}\right)$. For root $T$ consistent estimators this equality indicates that the difference between the residuals and the true innovations vanishes at a rate slightly slower than root $T$. Together with the i.i.d. assumption (see Assumption 4.2(a)) this hints towards resampling from the residuals to create the bootstrap samples. Details on the exact implementation are provided later.

There is another similarity between the residuals and innovations if an intercept is included in the vector autoregression. In this specific case, the residuals will have median zero for every cross-section. ${ }^{2}$ This observation stems from the fact that the absolute difference between the number of positive and negative residuals is bounded above by the number of residuals that are exactly zero (see theorem 3 on pages 16 and 17 of Bloomfield and Steiger (1983)). The number of exactly zero residuals is at least $M$.

We now provide the details on the implementation. The fixed design bootstrap treats the dependent variable, $\boldsymbol{z}_{t-1}$, as given. The bootstrap sample $\left\{\boldsymbol{y}_{t}^{*}\right\}$ is generated as $\boldsymbol{y}_{t}=$ $\left(\boldsymbol{I}_{K} \otimes \boldsymbol{z}_{t-1}^{\prime}\right) \widehat{\boldsymbol{b}}_{T}+\boldsymbol{u}_{t}^{*}$ where $\widehat{\boldsymbol{b}}_{T}$ denotes the parameter estimates ${ }^{3}$ and $\boldsymbol{u}_{t}^{*}$ is drawn with replacement from the residuals. The estimated parameters in the bootstrap sample are computed by solving the following analogue of Equation (4.3):

$$
\widehat{\gamma}_{T}^{*}=\arg \min _{\boldsymbol{\gamma} \in \Gamma} \frac{1}{T} \sum_{t=1}^{T} \sum_{k=1}^{K}\left|y_{k t}^{*}-\boldsymbol{z}_{t-1}^{\prime}\left(\boldsymbol{R}_{k} \gamma+\boldsymbol{r}_{k}\right)\right|:=\arg \min _{\boldsymbol{\gamma} \in \Gamma} S_{T}^{*}(\boldsymbol{\gamma}) .
$$

Note that superscripts stars are used to refer to quantities calculated within the fixed design residual bootstrap. The bootstrap algorithm is stated in Figure 4.1. In anticipation of future results on linear hypothesis testing, we have phrased these algorithms in terms of right-tailed tests. Different tests would require the usual modifications.

\footnotetext{
${ }^{2}$ This result has a well-known counterpart in least squares estimation. Here, the inclusion of a constant ensures that the residuals have a sample mean of zero. Freedman (1981) showed that mean zero (or centered) residuals are important for the validity of the bootstrap in the least squares setting.

${ }^{3}$ To keep the notation concise, we will not specify exactly which parameter estimates have been used. We refer to Remark 4.5 for details.
} 


\section{Fixed Design Residual Bootstrap}

STEP 1: Given a presample $\boldsymbol{y}_{1}, \boldsymbol{y}_{2}, \ldots, \boldsymbol{y}_{p}$, compute the parameter estimates $\widehat{\boldsymbol{b}}_{T}$ and the test statistic of interest, say $T(Y)$.

STEP 2: Obtain the residual vectors

$$
\widehat{\boldsymbol{u}}_{t}=\boldsymbol{y}_{t}-\left(\boldsymbol{I}_{K} \otimes \boldsymbol{z}_{t-1}^{\prime}\right) \widehat{\boldsymbol{b}}_{T}, \quad \text { for } t=p+1, p+2, \ldots, T \text {. }
$$

STEP 3: Construct bootstrap samples as $\boldsymbol{y}_{t}^{*}=\left(\boldsymbol{I}_{K} \otimes \boldsymbol{z}_{t-1}^{\prime}\right) \widehat{\boldsymbol{b}}_{T}+\boldsymbol{u}_{t}^{*}$, using the observed $\boldsymbol{z}_{t-1}$ and resampling $\boldsymbol{u}_{t}^{*}$ with replacement from $\left\{\widehat{\boldsymbol{u}}_{p+1}, \widehat{\boldsymbol{u}}_{p+2}, \ldots, \widehat{\boldsymbol{u}}_{T}\right\}$.

STEP 4: Repeat the previous step $B$ times and select the bootstrap critical value, say $c_{\alpha, B}^{*}$, as the $(1-\alpha)$-quantile of the ordered bootstrap test statistics $T\left(\boldsymbol{Y}^{*}\right)$. Reject the null hypothesis if $T(\boldsymbol{Y})>c_{\alpha, B}^{*}$.

Figure 4.1: The bootstrap algorithm for the fixed design residual bootstrap.

Remark 4.2 Up to this point we have only discussed the boostrap with fixed design. This method was also employed by Gonçalves and Kilian (2007). There are several references that use a recursive scheme to bootstrap autoregressions (e.g. Kreiss (1997) and Paparoditis (1996)). The implementation of a recursive design bootstrap would be as follows: given a presample, generate the bootstrap pseudodata from the recursion $\boldsymbol{y}_{t}^{\dagger}=\widehat{\boldsymbol{\nu}}_{T}+\widehat{\boldsymbol{A}}_{1, T} \boldsymbol{y}_{t-1}^{\dagger}+\cdots+\widehat{\boldsymbol{A}}_{p, T} \boldsymbol{y}_{t-p}^{\dagger}+\boldsymbol{u}_{t}^{\dagger}$ where the intercept vector $\widehat{\boldsymbol{\nu}}_{T}$ and autoregressive matrices $\left\{\widehat{\boldsymbol{A}}_{1, T}, \widehat{\boldsymbol{A}}_{2, T}, \ldots, \widehat{\boldsymbol{A}}_{p, T}\right\}$ are implied by the estimated parameters, Equation (4.2) and the relation $\widehat{\boldsymbol{b}}_{T}=\operatorname{vec}\left(\widehat{\boldsymbol{B}}_{T}\right)$. The bootstrap innovations $\boldsymbol{u}_{t}^{\dagger}$ are (as usual) an i.i.d. sample from the discrete uniform distribution on the residuals. We refer to Remark 4.7 and Appendix 4.C. The Monte Carlo results in the latter appendix indicate that the recursive design bootstrap does not perform as well as the fixed design bootstrap.

The following theorem shows that the asymptotic distribution of the parameters is well approximated by the fixed design residual bootstrap. All proofs are presented in the Appendix.

\section{Theorem 4.2 (Bootstrap VAlidity OF THE Estimators)}

Define $\boldsymbol{\Sigma}_{\text {asymp }}=\frac{1}{4}\left[\boldsymbol{R}^{\prime}(\boldsymbol{\Xi} \otimes \boldsymbol{\Lambda}) \boldsymbol{R}\right]^{-1} \boldsymbol{R}^{\prime}(\mathrm{E}(\boldsymbol{\Psi}) \otimes \boldsymbol{\Lambda}) \boldsymbol{R}\left[\boldsymbol{R}^{\prime}(\boldsymbol{\Xi} \otimes \boldsymbol{\Lambda}) \boldsymbol{R}\right]^{-1}$. If Assumptions 4.1-4.5 hold, then the conditional distribution of $\sqrt{T}\left(\widehat{\gamma}_{T}^{*}-\widehat{\gamma}_{T}\right)$ converges weakly to a normally distributed random vector with mean $\mathbf{0}$ and covariance matrix $\boldsymbol{\Sigma}_{\text {asymp. }}$. 
A comparison of the limiting distribution in Theorem 4.1(b) with that of Theorem 4.2 shows that they are the same. The bootstrap resampling method therefore correctly replicates the limiting distribution of the parameters. For any component of $\gamma_{0}$ this immediately justifies the use of the quantiles of the bootstrap distribution to construct percentile confidence intervals with the asymptotically correct coverage probability.

Remark 4.3 The bootstrap validity result, Theorem 4.2, is also interesting in view of impulse response analysis. A comparison to the least squares estimation (see Kilian (1998)) reveals that bootstrap consistency for the autoregressive parameters is the main requirement for valid inference on impulse responses. ${ }^{4}$

These percentile confidence intervals alone will not allow us to jointly test a hypothesis that specifies a set of linear constraints (for instance for sequential testing to determine the lag order). Suppose we would like to test whether $\boldsymbol{b}_{0}=\boldsymbol{R} \gamma_{0}+\boldsymbol{r}$, or equivalently whether $\boldsymbol{C} \boldsymbol{b}_{0}=\boldsymbol{c}$ for a given $(J \times K(K p+1))$ matrix $\boldsymbol{C}$ and a given $J$ vector $\boldsymbol{c}$ with $J=K(K p+1)-M .^{5}$ To test such an hypothesis, we propose the following two test statistics:

$$
\widetilde{W}_{T}=\left(\widehat{C b}_{T}^{U}-\boldsymbol{c}\right)^{\prime}\left(\widehat{C \boldsymbol{b}}_{T}^{U}-\boldsymbol{c}\right), \quad \widetilde{S}_{T}=\boldsymbol{g}_{T}\left(\widehat{\boldsymbol{b}}_{T}^{R}\right)^{\prime} \boldsymbol{g}_{T}\left(\widehat{\boldsymbol{b}}_{T}^{R}\right)
$$

with $\widehat{\boldsymbol{b}}_{T}^{R}$ and $\widehat{\boldsymbol{b}}_{T}^{U}$ being respectively the restricted and unrestricted parameter estimates and $\boldsymbol{g}_{T}(\boldsymbol{b})=-\frac{1}{\sqrt{T}} \sum_{t=1}^{T}\left(\operatorname{sgn}\left(\boldsymbol{y}_{t}-\left(\boldsymbol{I}_{k} \otimes \boldsymbol{z}_{t-1}^{\prime}\right) \boldsymbol{b}\right) \otimes \boldsymbol{I}_{K p+1}\right) \boldsymbol{z}_{t-1}$. Both test statistics should be readily recognized as modified versions of the Wald and LM test of Chapter 3. The modification consists of leaving out the weighting by the inverse asymptotic covariance matrix. ${ }^{6}$ This change is necessary if we are unwilling to use density estimators. The price to pay for this modification is a non-pivotal limiting distribution. We refer to Remarks 4.4 and 4.6 for further comments on this point. Although asymptotically non-pivotal, these test statistics can nevertheless be used because the following theorem asserts the bootstrap validity of these tests.

\footnotetext{
${ }^{4}$ The result would be immediate for the impulse responses without orthogonalization because these impulse responses depend on the autoregressive parameters only. LAD inference on orthogonalized impulse responses would be more involved because one would have to determine/estimate the (rotation) matrix that makes the innovations uncorrelated.

${ }^{5}$ It has been demonstrated in footnote 9 on page 65 that both formulations are equivalent.

${ }^{6}$ Let us emphasize that these Wald and LM tests are not the test statistics as implied by a likelihood framework. We therefore speak of modified versions of these tests or type of tests.
} 


\section{Theorem 4.3 (Bootstrap VALIDITy OF ThE TESTs)}

Let Assumptions 4.1-4.5 hold and refer to the bootstrap routine outlined in Figures 4.1 and 4.2 .

(a) Consider the Wald type test statistic: $\widetilde{W}_{T}=\left(\boldsymbol{C} \widehat{\boldsymbol{b}}_{T}^{U}-\boldsymbol{c}\right)^{\prime}\left(\boldsymbol{C} \widehat{\boldsymbol{b}}_{T}^{U}-\boldsymbol{c}\right)$. Let $c_{\alpha, B, W}^{*}$ be defined as the $(1-\alpha)$-quantile of the B bootstrap samples of the test statistic $\widetilde{W}_{T}^{*}=$ $\left(\boldsymbol{C} \widehat{\boldsymbol{b}}_{T}^{U, *}-\boldsymbol{c}\right)^{\prime}\left(\boldsymbol{C} \widehat{\boldsymbol{b}}_{T}^{U, *}-\boldsymbol{c}\right)$, with $\widehat{\boldsymbol{b}}_{T}^{U, *}$ the unrestricted parameter estimates in the fixed design bootstrap sample. For $c_{\alpha, W}^{*}=\operatorname{plim}_{B \rightarrow \infty}^{*} c_{\alpha, B, W}^{*}$, we have $\operatorname{Pr}\left(\widetilde{W}_{T}>c_{\alpha, W}^{*}\right) \rightarrow \alpha$.

(b) Consider the Score type test statistic: $\widetilde{\boldsymbol{S}}_{T}=\boldsymbol{g}_{T}\left(\widehat{\boldsymbol{b}}_{T}^{R}\right)^{\prime} \boldsymbol{g}_{T}\left(\widehat{\boldsymbol{b}}_{T}^{R}\right)$. Now let $c_{\alpha, B, S}^{*}$ be defined as the $(1-\alpha)$-quantile of the B bootstrap samples of the test statistic $\widetilde{S}_{T}^{*}=$ $\boldsymbol{g}_{T}^{*}\left(\widehat{\boldsymbol{b}}_{T}^{R, *}\right)^{\prime} \boldsymbol{g}_{T}^{*}\left(\widehat{\boldsymbol{b}}_{T}^{R, *}\right)$, with $\boldsymbol{g}_{T}^{*}(\boldsymbol{b})=-\frac{1}{\sqrt{T}} \sum_{t=1}^{T}\left(\operatorname{sgn}\left(\boldsymbol{y}_{t}^{*}-\left(\boldsymbol{I}_{k} \otimes \boldsymbol{z}_{t-1}^{\prime}\right) \boldsymbol{b}\right) \otimes \boldsymbol{I}_{K p+1}\right) \boldsymbol{z}_{t-1}$ and $\widehat{\boldsymbol{b}}_{T}^{R, *}$ the restricted parameter estimates in the fixed design bootstrap sample. For $c_{\alpha, S}^{*}=\operatorname{plim}_{B \rightarrow \infty}^{*} c_{\alpha, B, S}^{*}$, we have $\operatorname{Pr}\left(\widetilde{S}_{T}>c_{\alpha, S}^{*}\right) \rightarrow \alpha$.

Remark 4.4 The test statistics of Equation (4.5) are not asymptotically pivotal. This seems unavoidable if one is not willing to use a density estimator. The bootstrap tests will have the correct asymptotic level but based on Beran (1987, 1988) we should not expect the bootstrap to provide any asymptotic refinement.

We can obtain asymptotically pivotal test statistics using a procedure mentioned by Hahn (1995). He suggests to directly resample the residuals to get consistent estimates of the density. That is, the median of the resampled residuals of cross-section $k$ will have an asymptotic variance equal to $\frac{1}{4\left[f_{k}(0)\right]^{2}}$. The variance of the medians in the bootstrap sample will thus provide an estimate for $f_{k}(0)$ and the Wald and Score test of Section 3.2 .2 can be computed. A double bootstrap procedure as described on page 190 of MacKinnon (2009) can be applied to obtain asymptotic refinements. See Appendix 4.D for the bootstrap algorithm and simulation results.

Remark 4.5 The bootstrap algorithm of Figure 4.1 can be based on the residuals from parameter estimates that either do or do not impose the linear restrictions. The preferred choice remains controversial in the literature. Davidson and Flachaire (2008) advocate the use of the constrained estimators because the reduced parameter uncertainty leads to less volatile residuals and thereby better size control. Paparoditis and Politis (2005) on the other hand show that the use of unconstrained residuals has advantages in terms of the power of the test. More details are given in the next section, especially in footnote 7. 


\subsection{Simulation Results}

We now report several simulation results on the finite-sample behavior of the bootstrap procedure. The data generating process (DGP) is identical to the one of Section 3.3 to allow for comparison. The VAR process was thus generated by

$$
\boldsymbol{y}_{t}=\left[\begin{array}{l}
0.02 \\
0.03
\end{array}\right]+\left[\begin{array}{ll}
0.5 & 0.1 \\
0.4 & 0.5
\end{array}\right] \boldsymbol{y}_{t-1}+\left[\begin{array}{cc}
\alpha & \alpha \\
0.25 & 0
\end{array}\right] \boldsymbol{y}_{t-2}+L \boldsymbol{u}_{t}, \quad L=\left[\begin{array}{cc}
0.3 & 0 \\
0 & 0.2
\end{array}\right]
$$

The largest absolute eigenvalue of the associated companion matrix is 0.769 . The stability condition of Assumption 4.1 is therefore satisfied. The innovation distributions $u_{i t}$ are i.i.d. distributed as: (1) $\mathrm{N}(0,1)$ random variates; (2) Laplace distributed innovations with unit variance; (3) Student-t random variables with degrees of freedom being either 3, 4, 5,10 or 20; and (4) a variety of mixtures of two normal distributions. These mixtures consist of draws from a $\mathrm{N}(0,1)$ distribution with probability $(1-\epsilon)$ and from a $\mathrm{N}(0,16)$ distribution with probability $\epsilon$. We generate these mixtures component-wise implying that for some given time $t, u_{1 t}$ can originate from the outlier distribution whereas $u_{2 t}$ is simulated from a standard normal.

The recursion of Equation (4.6) was started by setting unknown initial values equal to zero. The first thousand observations were discarded to diminish the influence of these initial values. The number of bootstrap replicates was $B=499$ and we use the constrained parameter estimates to compute the residuals for the bootstrap routines. ${ }^{7}$ All results are based on 1,000 Monte Carlo replications. For this number of replicates, the simulation standard errors for the rejection frequencies are at $\operatorname{most}^{\max _{0 \leq p \leq 1}} \sqrt{p(1-p) / 1000} \approx$ $1.58 \%$.

We will test whether the lag $\boldsymbol{y}_{t-2}$ is relevant in the equation for $y_{1 t}$, hence $\alpha=0$ under the null hypothesis. The empirical power was simulated using $\alpha=-0.1$ and $\alpha=-0.2{ }^{8}$ Tables 4.1 and 4.2 report simulation outcomes for the modified Wald, modified Lagrange Multiplier (LM) and Likelihood Ratio (LR) test. The bootstrap validity of the latter test

\footnotetext{
${ }^{7}$ Appendix 4.B displays simulation results for both restricted and unrestricted innovations. The observations by Davidson and Flachaire (2008) and Paparoditis and Politis (2005) (see Remark 4.5) are confirmed in our simulations. In our setting, the power gains from using unrestricted residuals are small so we decided to use constrained residuals in all further simulations.

${ }^{8}$ These negative values for $\alpha$ have little influence on the largest absolute root implying that stability is still ensured.
} 
statistic has not been formally established but the LR test has nevertheless been included for comparison. ${ }^{9}$ We observe the following:

1. The empirical size is either close to the nominal level of 5\% or approaches this level as the sample size increases.

2. The LAD based tests gain empirical power as the innovation distribution becomes more leptokurtic or as the probability $\epsilon$ increases. The same behavior was also reported for the test statistics of Chapter 3 that used kernel density estimation (see Section 3.3).

3. For every simulation design, the modified Lagrange Multiplier test has the lowest empirical power.

A comparison of the size and power properties of the bootstrap test statistics and the test statistics computed with kernel density estimation is shown in Table 4.3. The rejection frequencies using density estimation are reported between parentheses immediately below the corresponding bootstrap results. The Likelihood Ratio test was not asymptotically pivotal so no results from Chapter 3 can be reported for this test. It remains to compare the Wald and LM types of tests. There is no substantial difference between the empirical size of the two procedures. However, in terms of power we can see that the bootstrapped version of the Wald statistic always outperforms the Wald test with density estimation. Conversely, the Lagrange Multiplier test statistic always has higher empirical power with kernel density estimation. The overall comparison of the empirical power is for all simulation settings in favor of the $\widetilde{W}_{T}$ test statistic. We therefore recommend the use of the $\widetilde{W}_{T}$ test statistic.

Remark 4.6 At first sight, it might be counterintuitive that the standard Wald test statistic $W_{T}=T\left(\boldsymbol{C} \widehat{\boldsymbol{b}}_{T}^{U}-\boldsymbol{c}\right)^{\prime}\left[\boldsymbol{C} \widehat{\boldsymbol{H}}_{T}^{-1} \widehat{\boldsymbol{V}}_{T} \widehat{\boldsymbol{H}}_{T}^{-1} \boldsymbol{C}^{\prime}\right]^{-1}\left(\widehat{C \boldsymbol{b}}_{T}^{U}-\boldsymbol{c}\right)$ from Chapter 3 does not have the highest empirical power. That is, we would expect that the weighting with the inverse asymptotic covariance matrix is beneficial in terms of power. However, we should also realize that this asymptotic covariance matrix requires density estimation. These density estimates converge slowly and can thus be imprecise.

\footnotetext{
${ }^{9}$ We implemented the Likelihood Ratio test in the following way. We first compute the statistic $L R_{T}$ as in Theorem 3.6, that is $L R_{T}=\sum_{t=1}^{T} \sum_{k=1}^{K}\left|y_{k t}-\boldsymbol{z}_{t-1}^{\prime} \boldsymbol{E}_{k} \widehat{\boldsymbol{b}}_{T}^{R}\right|-\sum_{t=1}^{T} \sum_{k=1}^{K}\left|y_{k t}-\boldsymbol{z}_{t-1}^{\prime} \boldsymbol{E}_{k} \widehat{\boldsymbol{b}}_{T}^{U}\right|$. In the latter expression, $\boldsymbol{E}_{k}$ is the matrix consisting of the rows of $\boldsymbol{I}_{K(K p+1)}$ that belong to the $k^{\prime}$ th cross-section, or equivalently $\boldsymbol{E}_{k} \widehat{\boldsymbol{b}}_{T}^{U}$ is the parameter vector belonging to equation $k$. For the fixed design bootstrap we subsequently generate $B$ bootstrap samples and reject the null hypothesis if the realized value of $L R_{T}$ exceeds the $95 \%$ empirical quantile of the bootstrap realizations of $L R_{T}^{*}=\sum_{t=1}^{T} \sum_{k=1}^{K}\left|y_{k t}^{*}-\boldsymbol{z}_{t-1}^{\prime} \boldsymbol{E}_{k} \widehat{\boldsymbol{b}}_{T}^{*, R}\right|-\sum_{t=1}^{T} \sum_{k=1}^{K}\left|y_{k t}^{*}-\boldsymbol{z}_{t-1}^{\prime} \boldsymbol{E}_{k} \widehat{\boldsymbol{b}}_{T}^{*, U}\right|$.
} 
Remark 4.7 The same simulations were carried out using a recursive design, see Tables 4.5-4.7 in Appendix 4.C. Especially Table 4.7 allows for an easy comparison between the two residual bootstrap designs. We conclude from this table that the residual bootstrap with fixed design has better size control while showing hardly any differences in terms of empirical power (taking into account the simulation uncertainty). The bootstrap method with fixed design is thus preferred based on the current simulation results.

\subsection{Conclusion}

The contributions of this chapter are the introduction of: (1) the asymptotically valid fixed design residual bootstrap and (2) two test statistics for bootstrap testing linear hypotheses for the parameters of a VAR model estimated by least absolute deviations. These methods allow the researcher to conduct inference on the LAD coefficients without any sort of density estimation. Our procedures are therefore straightforward to implement and require no decision on either the bandwidth (as for kernel density estimation, see Silverman (1986)) or the subsample size (as for subsampling, see Chernozhukov and Fernández-Val (2005)).

The Monte Carlo study indicated that the residual bootstrap with fixed design has power similar to that of the recursive design residual bootstrap while offering better size control. The comparison of the bootstrap methods was thus decided in favor of the fixed design residual bootstrap. The comparison of the test statistics established a clear preference for the Wald type statistic because of its superior empirical power. Based on the current simulations, we advice practitioners to use the Wald type test statistic in combination with the fixed design residuals bootstrap.

The proof of the bootstrap validity of the Likelihood Ratio test would be a possible extension of this paper. The reported simulation outcomes are at least not obviously at odds with such a validity result. As a further extension one might try to relax the i.i.d. assumption on the error term and allow for conditional heteroskedasticity as in Gonçalves and Kilian (2007). This naturally hints towards a Wild bootstrap approach of which the properties would still have to be established. Such results would provide further LAD alternatives to existing least squares approaches. 


\begin{tabular}{cc||ccc|ccc|ccc} 
& \multicolumn{1}{c}{} & \multicolumn{3}{c}{$\boldsymbol{T}=\mathbf{1 0 0}$} & \multicolumn{3}{c|}{$\boldsymbol{T}=\mathbf{2 5 0}$} & \multicolumn{3}{c}{$\boldsymbol{T}=\mathbf{5 0 0}$} \\
& $\boldsymbol{\alpha}$ & Wald & LM & LR & Wald & LM & LR & Wald & LM & LR \\
\hline \hline \multirow{2}{*}{$\mathcal{N}(0,1)$} & 0 & 5.6 & 5.2 & 6.9 & 4.9 & 5.5 & 6.2 & 5.0 & 5.5 & 5.5 \\
& -0.1 & 14.7 & 8.9 & 14.7 & 28.9 & 16.5 & 25.7 & 45.1 & 22.8 & 38.8 \\
& -0.2 & 37.8 & 13.7 & 33.6 & 73.9 & 46.0 & 72.2 & 94.9 & 79.0 & 92.6 \\
\hline \multirow{3}{*}{ Laplace } & 0 & 4.6 & 4.7 & 5.4 & 4.9 & 5.0 & 5.1 & 5.1 & 4.3 & 4.7 \\
& -0.1 & 18.8 & 11.4 & 23.3 & 48.7 & 29.2 & 46.9 & 80.2 & 58.5 & 77.4 \\
& -0.2 & 58.2 & 29.6 & 60.6 & 95.6 & 76.9 & 95.0 & 100.0 & 98.7 & 99.7 \\
\hline \multirow{3}{*}{$(3)$} & 0 & 4.6 & 5.4 & 4.7 & 5.7 & 4.9 & 5.7 & 5.3 & 5.3 & 5.7 \\
& -0.1 & 20.6 & 11.5 & 23.3 & 49.5 & 25.7 & 46.1 & 78.8 & 49.8 & 75.0 \\
& -0.2 & 61.4 & 27.3 & 65.3 & 95.5 & 70.3 & 94.0 & 100.0 & 96.7 & 100.0 \\
\hline \multirow{2}{*}{$\mathrm{t}(4)$} & 0 & 6.5 & 3.6 & 6.2 & 4.9 & 4.6 & 5.9 & 4.6 & 4.6 & 4.7 \\
& -0.1 & 19.0 & 11.3 & 22.7 & 39.2 & 21.8 & 38.4 & 67.1 & 40.6 & 62.8 \\
& -0.2 & 55.4 & 22.6 & 55.9 & 91.3 & 64.6 & 90.6 & 99.5 & 95.1 & 99.3 \\
\hline \multirow{2}{*}{$\mathrm{t}(5)$} & 0 & 4.9 & 5.1 & 5.7 & 5.0 & 5.7 & 4.7 & 4.3 & 5.7 & 5.4 \\
& -0.1 & 17.6 & 9.0 & 17.6 & 33.1 & 20.6 & 32.8 & 60.0 & 36.1 & 56.2 \\
& -0.2 & 47.8 & 23.2 & 47.8 & 88.6 & 61.2 & 85.0 & 98.9 & 91.3 & 98.5 \\
\hline \multirow{2}{*}{$\mathrm{t}(10)$} & 0 & 5.7 & 5.7 & 5.9 & 5.0 & 4.5 & 5.0 & 5.6 & 4.2 & 5.6 \\
& -0.1 & 16.5 & 7.1 & 14.8 & 30.9 & 16.7 & 27.8 & 50.2 & 30.4 & 46.2 \\
& -0.2 & 40.4 & 17.8 & 39.6 & 76.6 & 47.6 & 74.5 & 97.1 & 86.5 & 96.3 \\
\hline \multirow{2}{*}{$\mathrm{t}(20)$} & 0 & 3.9 & 4.3 & 4.9 & 6.0 & 5.5 & 6.3 & 5.2 & 5.6 & 6.3 \\
& -0.1 & 14.0 & 8.1 & 14.2 & 27.2 & 14.6 & 24.1 & 50.3 & 28.4 & 43.2 \\
& -0.2 & 40.8 & 16.3 & 39.5 & 76.1 & 47.7 & 73.6 & 96.6 & 81.4 & 95.0
\end{tabular}

Table 4.1: The empirical size and power (in percentages) of the three bootstrapped test statistics of Equation (4.5) for various innovation distributions and sample sizes. The desired nominal size is $5 \%$. The data was generated according to the model specified by Equation (4.6). Results are based on the fixed design residual bootstrap. 


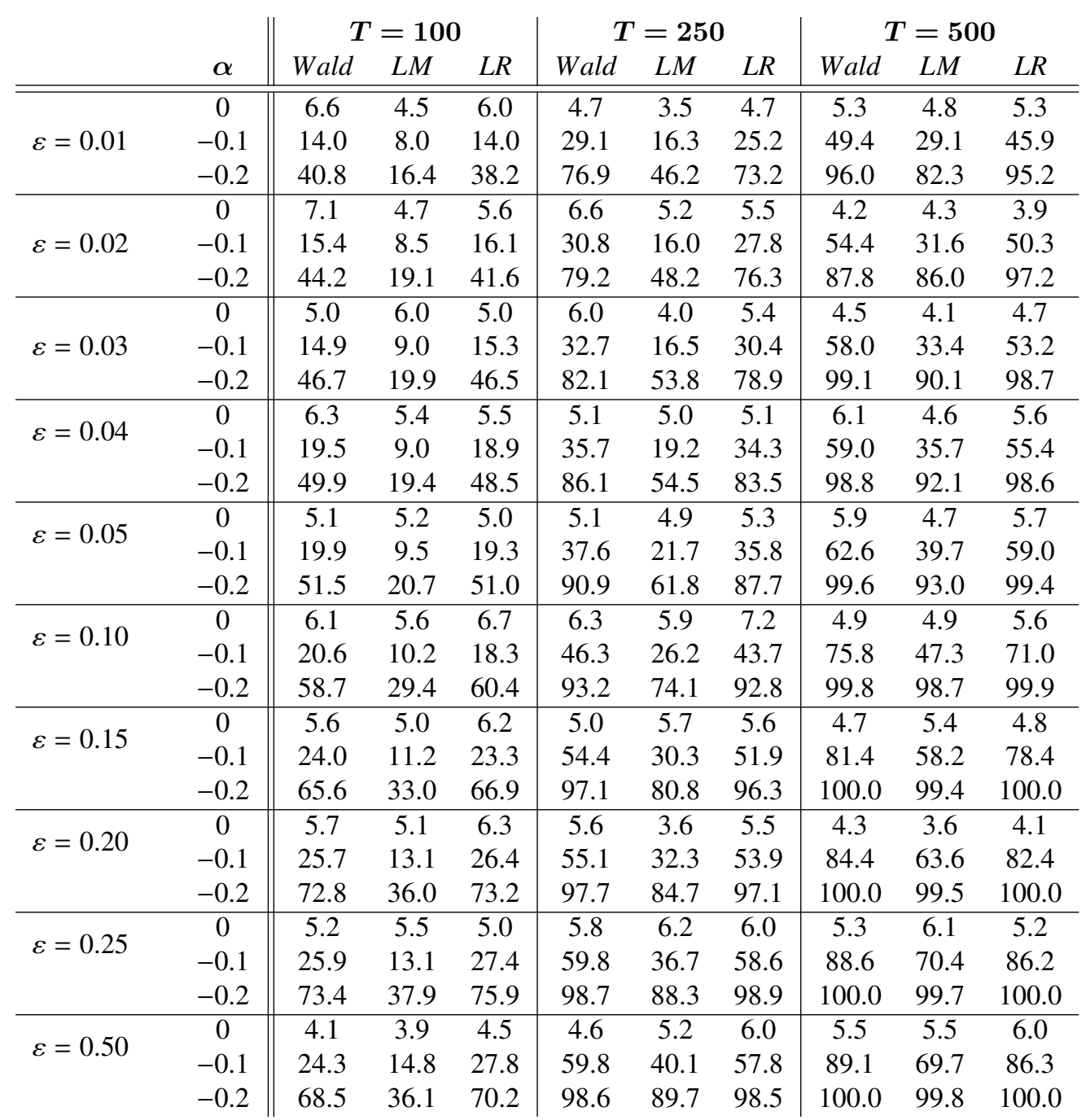

Table 4.2: The empirical size and power (in percentages) of the three bootstrapped test statistics of Equation (4.5) for a varying percentage of outliers in the mixture distribution and three sample sizes. The desired nominal size is 5\%. See Equation (4.6) for the data generating process. Results are based on the fixed design residual bootstrap. 


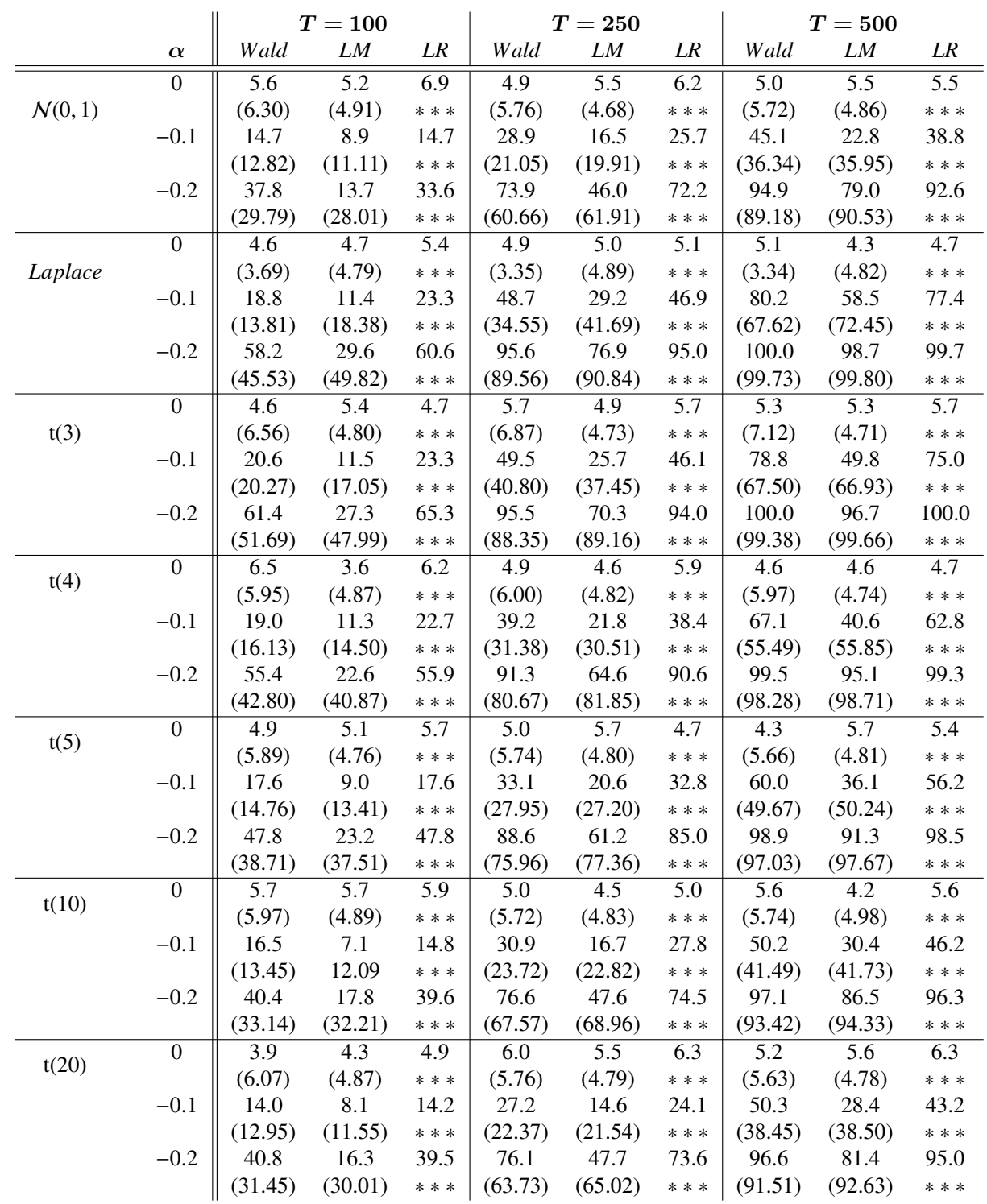

Table 4.3: The empirical rejection frequencies of the bootstrap test statistics (standard typesetting) and the test statistics from Chapter 3 based on kernel density estimation (between parentheses). The Likelihood Ratio statistic is not asymptotically pivotal and cannot be computed with kernel density estimators. 


\section{A Mathematical Appendix}

\section{A.1 Parameter Estimation}

The proofs use the metric $d_{r}^{p} . d_{r}^{p}$ is defined as a metric for probabilities in $\mathbb{R}^{p}$ w.r.t. the Euclidean norm $\|\cdot\|$. Explicitly, if $\mu$ and $v$ are probabilities in $\mathbb{R}^{p}$, then $d_{r}^{p}(\mu, v)$ is the infimum of $\left[\mathrm{E}\left(\|\boldsymbol{U}-\boldsymbol{V}\|^{r}\right)\right]^{1 / r}$ over all pairs of random vectors $\boldsymbol{U}$ and $\boldsymbol{V}$ where $\boldsymbol{U}$ has marginal law $\mu$ and $\boldsymbol{V}$ has marginal law $v \cdot{ }^{10}$ An exposition of several properties of the Mallows metric can be found in section 8 of Bickel and Freedman (1981). Since Mallows (1972), $d_{2}^{p}$ is known as the Mallows metric. Our proof uses elements from Bickel and Freedman (1981), Kreiss and Franke (1992), Herce (1996) and Moreno and Romo (2000). We will use $a \stackrel{o_{p}(1)}{=} b$ as a shorthand for $a=b+o_{p}(1)$. Additionally, we define the following cumulative distribution functions (CDFs). The CDF of the innovations $\boldsymbol{u}_{t}$ is denoted $F$, the distribution of the (unobserved) innovation in the sample is $F_{T}$, and the empirical distribution of the residuals $\widehat{\boldsymbol{u}}_{t}$ will be denoted $\widehat{F}_{T}$.

We start the proof with the following lemma.

\section{Lemma 4.1}

For $\boldsymbol{\eta}_{t}=\sum_{k=1}^{K} \boldsymbol{R}_{k}^{\prime} \boldsymbol{z}_{t-1} \operatorname{sgn}\left(u_{k t}\right)$ and $\boldsymbol{\eta}_{t}^{*}=\sum_{k=1}^{K} \boldsymbol{R}_{k}^{\prime} \boldsymbol{z}_{t-1} \operatorname{sgn}\left(u_{k t}^{*}\right)$, we have

$$
d_{2}^{M}\left(\frac{1}{\sqrt{T}} \sum_{t=1}^{T} \boldsymbol{\eta}_{t}, \frac{1}{\sqrt{T}} \sum_{t=1}^{T} \boldsymbol{\eta}_{t}^{*}\right)=o_{p}(1)
$$

\section{Proof}

We follow the approach by Kreiss and Franke (1992). Write $m_{k}^{*}$ for the expectation of $\operatorname{sgn}\left(\tilde{u}_{k t}^{*}\right)$ under $\widehat{F}_{n}$. Using $\operatorname{Esgn}\left(u_{k t}\right)=0$ (Assumption 4.2(c)), we have

$$
\begin{aligned}
\left|m_{k}^{*}\right|= & \left|\frac{1}{n} \sum_{t=1}^{T} \operatorname{sgn}\left(\widehat{u}_{k t}\right)-\operatorname{Esgn}\left(u_{k t}\right)\right| \\
& \leq \frac{1}{n} \sum_{t=1}^{T}\left|\operatorname{sgn}\left(\widehat{u}_{k t}\right)-\operatorname{sgn}\left(u_{k t}\right)\right|+\left|\frac{1}{n} \sum_{t=1}^{T} \operatorname{sgn}\left(u_{k t}\right)-\operatorname{Esgn}\left(u_{k t}\right)\right| .
\end{aligned}
$$

\footnotetext{
${ }^{10}$ As in Freedman (1981) and Kreiss and Franke (1992), we will use $d_{r}^{p}(\boldsymbol{U}, \boldsymbol{V})$ as a shorthand notation for $d_{r}^{p}\left(F_{\boldsymbol{U}}, F_{\boldsymbol{V}}\right)$ where $F_{\boldsymbol{U}}$ and $F_{\boldsymbol{V}}$ denote the laws of $\boldsymbol{U}$ and $\boldsymbol{V}$. Moreover, the superscript $p$ will be omitted if $p=1$.
} 
The second term is $o_{p}(1)$ by the law of large numbers for i.i.d. sequences. Additionally, by Lemma 4(iii) of Arcones (1996), i.e. $|\operatorname{sgn}(a+b)-\operatorname{sgn}(a)| \leq 2 \mathbb{1}_{\{|a| \leq|b|\}}$,

$$
\begin{gathered}
\frac{1}{T} \sum_{t=1}^{T} \mathrm{E}\left|\operatorname{sgn}\left(\widehat{u}_{k t}\right)-\operatorname{sgn}\left(u_{k t}\right)\right| \leq \frac{2}{T} \sum_{t=1}^{T} \operatorname{Pr}\left(\left|u_{k t}\right| \leq\left|z_{t-1}^{\prime} \boldsymbol{R}_{k}\left(\gamma_{0}-\widehat{\gamma}_{T}\right)\right|\right) \\
\leq 2 C \mathrm{E}\left\|z_{t-1}\right\|\left\|\boldsymbol{R}_{k}\right\| \delta+\operatorname{Pr}\left(\left\|\widehat{\gamma}_{T}-\gamma_{0}\right\|>\delta\right),
\end{gathered}
$$

where $C$ denotes a constant which bounds the density from above (see Assumption 4.2(d)). The first term in Equation (4.7) is thus also $o_{p}(1)$ since $\left\|\widehat{\gamma}_{T}-\gamma_{0}\right\|=O_{p}\left(T^{-1 / 2}\right)$ and $\delta$ can be taken arbitrarily small. We conclude that $m_{k}^{*}=o_{p}(1)$.

Now back to the result we would like to establish. Consider i.i.d. pairs $\left(\tilde{\boldsymbol{u}}_{t}, \tilde{\boldsymbol{u}}_{t}^{*}\right)$ with marginal distributions $F_{T}$ and $\widehat{F}_{T}$ and let the stated infima be taken over all joint distributions with these marginals, then (with $\|\cdot\|$ denoting the $L_{2}$ norm conditional on the sample),

$$
\begin{gathered}
d_{2}^{M}\left(\frac{1}{\sqrt{T}} \sum_{t=1}^{T} \boldsymbol{\eta}_{t}^{*}, \frac{1}{\sqrt{T}} \sum_{t=1}^{T} \boldsymbol{\eta}_{t}\right)=\inf \left\|\frac{1}{\sqrt{T}} \sum_{t=1}^{T} \sum_{k=1}^{K} \boldsymbol{R}_{k}^{\prime} \boldsymbol{z}_{t-1}\left[\operatorname{sgn}\left(\tilde{u}_{k t}^{*}\right)-\operatorname{sgn}\left(\tilde{u}_{k t}\right)\right]\right\| \\
=\inf \| \frac{1}{\sqrt{T}} \sum_{t=1}^{T} \sum_{k=1}^{K} \boldsymbol{R}_{k}^{\prime} \boldsymbol{z}_{t-1}\left[\operatorname{sgn}\left(\tilde{u}_{k t}^{*}\right)-\operatorname{sgn}\left(\tilde{u}_{k t}\right)-m_{k}^{*}\right] \\
+\sum_{k=1}^{K} m_{k}^{*} \boldsymbol{R}_{k}^{\prime}\left(\frac{1}{\sqrt{T}} \sum_{t=1}^{T} \boldsymbol{z}_{t-1}\right) \|:=I+I I .
\end{gathered}
$$

For the first term, we make use of the fact that all cross terms vanish under the expectation because $\left(\tilde{\boldsymbol{u}}_{t}, \tilde{\boldsymbol{u}}_{t}^{*}\right)$ is independent of $\boldsymbol{z}_{t-1}$ and $\operatorname{sgn}\left(\tilde{u}_{k t}^{*}\right)-\operatorname{sgn}\left(\tilde{u}_{k t}\right)-m_{k}^{*}$ has mean zero. So,

$$
\begin{aligned}
& E\left\|\frac{1}{\sqrt{T}} \sum_{t=1}^{T} \sum_{k=1}^{K} \boldsymbol{R}_{k}^{\prime} \boldsymbol{z}_{t-1}\left[\operatorname{sgn}\left(\tilde{u}_{k t}^{*}\right)-\operatorname{sgn}\left(\tilde{u}_{k t}\right)-m_{k}^{*}\right]\right\|^{2} \\
& \leq \frac{1}{T} \mathrm{E} \sum_{t=1}^{T} \sum_{k=1}^{K} \sum_{k=1}^{K}\left[\operatorname{sgn}\left(\tilde{u}_{k t}^{*}\right)-\operatorname{sgn}\left(\tilde{u}_{k t}\right)-m_{k}^{*}\right]\left[\operatorname{sgn}\left(\tilde{u}_{k t}^{*}\right)-\operatorname{sgn}\left(\tilde{u}_{k t}\right)-m_{\kappa}^{*}\right] \boldsymbol{z}_{t-1}^{\prime} \boldsymbol{R}_{k} \boldsymbol{R}_{k}^{\prime} \boldsymbol{z}_{t-1} \\
& \leq\|\boldsymbol{R}\|^{2} \mathrm{E}\left\|\boldsymbol{z}_{t-1}\right\|^{2} \sum_{k=1}^{K} \sum_{k=1}^{K} \mathrm{E}\left[\operatorname{sgn}\left(\tilde{u}_{k t}^{*}\right)-\operatorname{sgn}\left(\tilde{u}_{k t}\right)-m_{k}^{*}\right]\left[\operatorname{sgn}\left(\tilde{u}_{k t}^{*}\right)-\operatorname{sgn}\left(\tilde{u}_{k t}\right)-m_{\kappa}^{*}\right],
\end{aligned}
$$

using weak stationarity. We conclude that $I=o_{p}(1)$ using: $\left|\operatorname{sgn}\left(\tilde{u}_{\kappa t}^{*}\right)-\operatorname{sgn}\left(\tilde{u}_{\kappa t}\right)-m_{\kappa}^{*}\right| \leq$ $2+\left|m_{\kappa}^{*}\right|, m_{k}^{*}=o_{p}(1)$ and an argument similar to Equation (4.8). For the second term, 
we note that $I I \leq \sum_{k=1}^{K} \mid m_{k}^{*}\left\|\boldsymbol{R}_{k}\right\| \mathrm{E}\left\|\frac{1}{\sqrt{T}} \sum_{t=1}^{T} \boldsymbol{z}_{t-1}\right\|=o_{p}(1)$ since $m_{k}^{*}=o_{p}(1)$ and the expectation is bounded due to summability of the autocovariances.

We now proof Theorem 4.2(a). By definition of the fixed design bootstrap procedure we can also write $S_{T}^{*}(\gamma)=\frac{1}{T} \sum_{t=1}^{T} \sum_{k=1}^{K}\left|u_{k t}^{*}-\boldsymbol{z}_{t-1}^{\prime} \boldsymbol{R}_{k}\left(\gamma-\widehat{\gamma}_{T}\right)\right|$ for the objective function in Equation (4.4). For fixed $\boldsymbol{\theta}$, the objective function

$$
Z_{T}^{*}(\boldsymbol{\theta})=\frac{1}{T} \sum_{t=1}^{T} \sum_{k=1}^{K}\left(\left|u_{k t}^{*}-\frac{\boldsymbol{z}_{t-1}^{\prime} \boldsymbol{R}_{k} \boldsymbol{\theta}}{\sqrt{T}}\right|-\left|u_{k t}^{*}\right|\right),
$$

is minimized at $\sqrt{T}\left(\widehat{\gamma}_{T}^{*}-\widehat{\gamma}_{T}\right)$. A short outline of the proof is as follows. We will show that $Z_{T}^{*}(\boldsymbol{\theta})$ converges in distribution to a quadratic form in $\boldsymbol{\theta}$. The minimizer of this quadratic form is easily computed and its limiting distribution coincides with the limiting distribution of $\sqrt{T}\left(\widehat{\gamma}_{T}^{*}-\widehat{\gamma}_{T}\right)$ by the convexity lemma on page 193 of Pollard (1991). We will subsequently see that this limiting distribution coincides with the limiting distribution of $\sqrt{T}\left(\widehat{\gamma}_{T}-\gamma_{0}\right)$.

See Equation (4.11), the equality $|a-b|-|a|=-\operatorname{sgn}(a) b+2(b-a)\left[\mathbb{1}_{\{0<a<b\}}-\mathbb{1}_{\{b<a<0\}}\right]$ with $a=u_{k t}^{*}$ and $b=\frac{\boldsymbol{z}_{t-1}^{\prime} \boldsymbol{R}_{k} \boldsymbol{\theta}}{\sqrt{T}}$ splits $Z_{T}^{*}(\boldsymbol{\theta})$ into three contributions

$$
\begin{aligned}
& Z_{T}^{*}(\boldsymbol{\theta})=-\frac{\boldsymbol{\theta}^{\prime}}{\sqrt{T}} \sum_{t=1}^{T} \sum_{k=1}^{K} \boldsymbol{R}_{k}^{\prime} \boldsymbol{z}_{t-1} \operatorname{sgn}\left(u_{k t}^{*}\right) \\
& +2 \sum_{t=1}^{T} \sum_{k=1}^{K}\left(\frac{\boldsymbol{z}_{t-1}^{\prime} \boldsymbol{R}_{k} \boldsymbol{\theta}}{\sqrt{T}}-u_{k t}^{*}\right) \mathbb{1}_{\left\{0<u_{k t}^{*}<\frac{\boldsymbol{z}_{t-1}^{\prime} \boldsymbol{R}_{k} \boldsymbol{\theta}}{\sqrt{T}}\right\}} \\
& -2 \sum_{t=1}^{T} \sum_{k=1}^{K}\left(\frac{\boldsymbol{z}_{t-1}^{\prime} \boldsymbol{R}_{k} \boldsymbol{\theta}}{\sqrt{T}}-u_{k t}^{*}\right) \mathbb{1}_{\left\{\frac{z_{t-1}^{\prime} \boldsymbol{R}_{k} \boldsymbol{\theta}}{\sqrt{T}}<u_{k t}^{*}<0\right\}}:=I+I I+I I I .
\end{aligned}
$$

The first term can be written as $I=-\boldsymbol{\theta}^{\prime}\left(\frac{1}{\sqrt{T}} \sum_{t=1}^{T} \boldsymbol{\eta}_{t}^{*}\right)$. By Lemma 8.3 of Bickel and Freedman (1981), convergence to zero in $d_{r}^{p}$ implies identical limiting distributions. Now recall our result from Lemma 4.1, i.e. $d_{2}^{M}\left(\frac{1}{\sqrt{T}} \sum_{t=1}^{T} \boldsymbol{\eta}_{t}, \frac{1}{\sqrt{T}} \sum_{t=1}^{T} \boldsymbol{\eta}_{t}^{*}\right)=o_{p}(1)$. Since $\frac{1}{\sqrt{T}} \sum_{t=1}^{T} \boldsymbol{\eta}_{t} \rightarrow_{d} \mathrm{~N}\left(\mathbf{0}, \boldsymbol{R}^{\prime}(\mathrm{E}(\boldsymbol{\Psi}) \otimes \boldsymbol{\Lambda}) \boldsymbol{R}\right)$ as in 'Derivation of the Central Limit Theorem' on page 92, we must also have $I \rightarrow_{d}-\boldsymbol{\theta}^{\prime} \boldsymbol{X}$ with $\boldsymbol{X} \sim \mathrm{N}\left(\mathbf{0}, \boldsymbol{R}^{\prime}(\mathrm{E}(\boldsymbol{\Psi}) \otimes \boldsymbol{\Lambda}) \boldsymbol{R}\right)$. 
The second term has the form $I I=2 \sum_{t=1}^{T} \sum_{k=1}^{K} V_{t T, k}(\boldsymbol{\theta})$. We consider the following truncated version of $V_{t T, k}$ indicated with a superscript $m$,

$$
\begin{aligned}
V_{t T, k}^{m} & =V_{t T, k} \mathbb{1}_{\left\{\frac{z_{t-1}^{\prime} \boldsymbol{R}_{k} \boldsymbol{\theta}}{\sqrt{T}} \leq m\right\}}=\left(\frac{\boldsymbol{z}_{t-1}^{\prime} \boldsymbol{R}_{k} \boldsymbol{\theta}}{\sqrt{T}}-u_{k t}^{*}\right) \mathbb{1}_{\left\{0<u_{k t}^{*}<\frac{\boldsymbol{z}_{t-1}^{\prime} \boldsymbol{R}_{k} \boldsymbol{\theta}}{\sqrt{T}}\right\}} \mathbb{1}_{\left\{\frac{\boldsymbol{z}_{t-1}^{\prime} \boldsymbol{R}_{k} \boldsymbol{\theta}}{\sqrt{T}} \leq m\right\}} \\
& \left.=\left(A_{t T, k}^{m}-u_{k t}^{*}\right) \mathbb{1}_{\left\{0<u_{k t}^{*}\right.} \frac{\boldsymbol{z}_{t-1}^{\prime} \boldsymbol{R}_{k} \boldsymbol{\theta}}{\sqrt{T}}\right\} \mathbb{1}_{\left\{\frac{\boldsymbol{z}_{t-1}^{\prime} \boldsymbol{R}_{k} \theta}{\sqrt{T}} \leq m\right\}},
\end{aligned}
$$

with $A_{t T, k}^{m}=\left(\frac{\boldsymbol{z}_{t-1}^{\prime} \boldsymbol{R}_{k} \boldsymbol{\theta}}{\sqrt{T}}\right) \mathbb{1}_{\left\{0<\frac{\boldsymbol{z}_{t-1}^{\prime} \boldsymbol{R}_{k} \boldsymbol{\theta}}{\sqrt{T}} \leq m\right\}}$. Let $\mu_{t T, k}^{m}$ denote the conditional expectation of $V_{t T, k}^{m}$ given the sample and $\left\{\boldsymbol{u}_{t-1}^{*}, \boldsymbol{u}_{t-2}^{*}, \ldots\right\}$, then

$$
\begin{aligned}
\mu_{t T, k}^{m} & =\int_{0}^{A_{t T, k}^{m}}\left(A_{t T, k}^{m}-x\right) d \widehat{F}_{T, k}(x)=\int_{0}^{A_{t T, k}^{m}} \int_{x}^{A_{t T, k}^{m}} d s d \widehat{F}_{T, k}(x) \\
& =\int_{0}^{A_{t T, k}^{m}} \int_{0}^{s} d \widehat{F}_{T, k}(x) d s=\int_{0}^{A_{t T, k}^{m}}\left(\widehat{F}_{T, k}(s)-\widehat{F}_{T, k}(0)\right) d s,
\end{aligned}
$$

where $\widehat{F}_{T, k}$ is the marginal CDF for the $k^{\prime}$ th argument of $\widehat{F}_{T}$. Similar to Boldin (1983), it can be shown that $\sup _{x} \sqrt{T}\left(\widehat{F}_{T, k}(x)-F_{T, k}(x)\right)=o_{p}(1)$ where $F_{T, k}$ is the empirical CDF based on the (unobserved) true innovations. Combined with the Glivenko-Cantelli theorem, we have $\sup _{x}\left(\widehat{F}_{T, k}(x)-F_{k}(x)\right)=o_{p}(1)$ with $F_{k}$ denoting the (marginal) CDF of $u_{k t}$. Since $0<A_{t T, k}^{m} \leq m$, also

$$
\begin{aligned}
\mu_{t T, k}^{m} & \stackrel{o_{p}(1)}{=} \int_{0}^{A_{t T, k}^{m}}\left(F_{k}(s)-F_{k}(0)\right) d s=\frac{1}{T} \int_{0}^{\sqrt{T} A_{t T, k}^{m}} v\left[\frac{F_{k}(v / \sqrt{T})-F_{k}(0)}{v / \sqrt{T}}\right] d v \\
& \stackrel{o_{p}(1)}{=} \frac{1}{T} \int_{0}^{\sqrt{T} A_{t T, k}^{m}} v f_{k}(0) d v=\frac{1}{2 T} f_{k}(0)\left[\sqrt{T} A_{t T, k}^{m}\right]^{2} \\
& =\frac{1}{2 T} f_{k}(0) \boldsymbol{\theta}^{\prime} \boldsymbol{R}_{k}^{\prime} \boldsymbol{z}_{t-1} \boldsymbol{z}_{t-1}^{\prime} \boldsymbol{R}_{k} \boldsymbol{\theta} \mathbb{1}_{\left\{0<\frac{\boldsymbol{z}_{t-1}^{\prime} \boldsymbol{R}_{k} \boldsymbol{\theta}}{\sqrt{T}} \leq m\right\}},
\end{aligned}
$$

using that fact that the term between square brackets is a difference quotient and a change of variables from $s$ to $v=s \sqrt{T}$. Now define

$$
\mu_{T, k}^{m}=\sum_{t=1}^{T} \mu_{t T, k}^{m}=\frac{1}{2} f_{k}(0) \boldsymbol{\theta}^{\prime} \boldsymbol{R}_{k}^{\prime}\left(\frac{1}{T} \sum_{t=1}^{T} \boldsymbol{z}_{t-1} \boldsymbol{z}_{t-1}^{\prime} \mathbb{1}_{\left\{0<\frac{\boldsymbol{z}_{t-1}^{\prime} \boldsymbol{R}_{k} \boldsymbol{\theta}}{\sqrt{T}} \leq m\right\}}\right) \boldsymbol{R}_{k} \boldsymbol{\theta}
$$

$\mu_{T, k}^{m}$ converges in probability to $\mu_{k}=\frac{1}{2} f_{k}(0) \boldsymbol{\theta}^{\prime} \boldsymbol{R}_{k}^{\prime} \mathrm{E}\left(\boldsymbol{z}_{t-1} \boldsymbol{z}_{t-1}^{\prime} \mathbb{1}_{\left\{0<\boldsymbol{z}_{t-1}^{\prime} \boldsymbol{R}_{k} \boldsymbol{\theta}\right\}}\right) \boldsymbol{R}_{k} \boldsymbol{\theta}$ by a LLN for triangular arrays. 
A martingale difference sequence argument is now used to relate the $V_{t T, k}^{m}$ to their conditional expectations. Define $V_{T, k}^{m}=\sum_{t=1}^{T} V_{t T, k}^{m}$. According to lemma 4.7 of White (1984)), the limiting distributions of $V_{T, k}^{m}$ and $\mu_{T, k}^{m}$ coincide, if we can show that $V_{T, k}^{m}-\mu_{T, k}^{m}=$ $\sum_{t=1}^{T}\left[V_{t T, k}^{m}-\mu_{t T, k}^{m}\right]=o_{p}(1)$. The term in square brackets is a martingale difference and by theorem 1 on page 171 of Pollard (1984) it suffices to show that $\sum_{t=1}^{T} \mathrm{E}\left(\left(V_{t T, k}^{m}\right)^{2} \mid \mathcal{F}_{t-1}\right) \stackrel{p}{\longrightarrow}$ 0 , where $\mathcal{F}_{t-1}$ denotes the $\sigma$-algebra generated by the sample and $\left\{\boldsymbol{u}_{t-1}^{*}, \boldsymbol{u}_{t-2}^{*}, \ldots\right\}$. Now note that $V_{t T, k}^{m} \leq A_{t T, k}^{m}$ and that $A_{t T, k}^{m}$ is $\mathcal{F}_{t-1}$ measurable, such that

$$
\sum_{t=1}^{T} \mathrm{E}\left(\left(V_{t T, k}^{m}\right)^{2} \mid \mathcal{F}_{t-1}\right) \leq \sum_{t=1}^{T} A_{t T, k}^{m} \mathrm{E}\left(V_{t T, k}^{m} \mid \mathcal{F}_{t-1}\right) \leq \mu_{T, k}^{m}\left(\max _{1 \leq t \leq T} A_{t T, k}^{m}\right)
$$

So the proof is complete if we can establish that $\max _{1 \leq t \leq T} A_{t T, k}^{m}$ can be made arbitrary small in probability. To show this, we write

$$
\begin{array}{r}
\operatorname{Pr}\left(\max _{1 \leq t \leq T} A_{t T, k}^{m}>\epsilon\right) \leq \operatorname{Pr}\left(\max _{1 \leq t \leq T}\left|\boldsymbol{z}_{t-1}^{\prime} \boldsymbol{R}_{k} \boldsymbol{\theta} / \sqrt{T}\right|>\epsilon\right)=\operatorname{Pr}\left(\bigcup _ { t = 1 } ^ { T } \left\{\frac{\left|\boldsymbol{z}_{t-1}^{\prime} \boldsymbol{R}_{k} \boldsymbol{\theta}\right|}{\sqrt{T}>m\})}\right.\right. \\
\quad \leq \sum_{t=1}^{T} \mathrm{E}\left(\mathbb{1}_{\left\{\frac{\left|z_{t-1}^{\prime} \boldsymbol{R}_{k} \boldsymbol{\theta}\right|}{\sqrt{T}}>m\right\}}\right) \leq \sum_{t=1}^{T} \mathrm{E}\left(\frac{\left(\boldsymbol{z}_{t-1}^{\prime} \boldsymbol{R}_{k} \boldsymbol{\theta}\right)^{2}}{T m^{2}} \mathbb{1}_{\left\{\frac{\boldsymbol{z}_{t-1}^{\prime} \boldsymbol{R}_{k} \boldsymbol{\theta}}{\sqrt{T}}>m\right\}}\right)=\frac{1}{m^{2}} \boldsymbol{\theta}^{\prime} \boldsymbol{R}_{k}^{\prime} \boldsymbol{\Lambda} \boldsymbol{R}_{k} \boldsymbol{\theta},
\end{array}
$$

which can indeed be made arbitrarily small by starting from a large enough $m$.

From the previous results we have $V_{T, k}(\boldsymbol{\theta})=\left(V_{T, k}(\boldsymbol{\theta})-V_{T, k}^{m}(\boldsymbol{\theta})\right)+\left(V_{T, k}^{m}(\boldsymbol{\theta})-\mu_{T, k}^{m}\right)+$ $\mu_{T, k}^{m} \stackrel{o_{p}(1)}{=} m_{k}$. It remains to show that the effect of truncation does not have an influence on the limiting distribution. That is, we should show that for arbitrary $\epsilon>0$,

$$
\lim _{m \rightarrow \infty} \limsup _{n \rightarrow \infty} \operatorname{Pr}\left(\left|V_{T, k}-V_{T, k}^{m}\right|>\epsilon\right)=0,
$$


with $V_{T, k}=\sum_{t=1}^{T} V_{t T, k}$. The probability in Equation (4.19) can be bounded above by

$$
\begin{aligned}
& \operatorname{Pr}\left(\left|V_{T, k}-V_{T, k}^{m}\right|>\epsilon\right)=\operatorname{Pr}\left(\sum_{t=1}^{T}\left(V_{t T, k}-V_{t T, k}^{m}\right)>\epsilon\right) \\
& \quad=\operatorname{Pr}\left(\sum_{t=1}^{T}\left(\frac{\boldsymbol{z}_{t-1}^{\prime} \boldsymbol{R}_{k} \boldsymbol{\theta}}{\sqrt{T}}-u_{k t}^{*}\right) \mathbb{1}_{\left\{0<u_{k t}^{*}<\frac{\boldsymbol{z}_{t-1}^{\prime} \boldsymbol{R}_{k} \boldsymbol{\theta}}{\sqrt{T}}\right\}} \mathbb{1}_{\left\{\frac{\boldsymbol{z}_{t-1}^{\prime} \boldsymbol{R}_{k} \boldsymbol{\theta}}{\sqrt{T}}>m\right\}}>\epsilon\right) \\
& \quad \leq \operatorname{Pr}\left(\sum_{t=1}^{T}\left(\frac{\boldsymbol{z}_{t-1}^{\prime} \boldsymbol{R}_{k} \boldsymbol{\theta}}{\sqrt{T}}-u_{k t}^{*}\right) \mathbb{1}_{\left\{0<u_{k t}^{*}<\frac{\boldsymbol{z}_{t-1}^{\prime} \boldsymbol{R}_{k} \boldsymbol{\theta}}{\sqrt{T}}\right\}} \mathbb{1}_{\left\{\frac{\boldsymbol{z}_{t-1}^{\prime} \boldsymbol{R}_{k} \boldsymbol{\theta}}{\sqrt{T}}>m\right\}}>0\right) \\
& \quad \leq \operatorname{Pr}\left(\bigcup_{t=1}^{T}\left\{\frac{\boldsymbol{z}_{t-1}^{\prime} \boldsymbol{R}_{k} \boldsymbol{\theta}}{\sqrt{T}}>m\right\}\right) \leq \sum_{t=1}^{T} \mathrm{E}\left(\mathbb{1}_{\left\{\frac{\boldsymbol{z}_{t-1}^{\prime} \boldsymbol{R}_{k} \boldsymbol{\theta}}{\sqrt{T}}>m\right\}}\right) \leq \frac{1}{m^{2}} \boldsymbol{\theta}^{\prime} \boldsymbol{R}_{k}^{\prime} \boldsymbol{\Lambda} \boldsymbol{R}_{k} \boldsymbol{\theta},
\end{aligned}
$$

using the same steps as in Equation (4.18). The requirement of Equation (4.19) is now seen to hold because

$$
\lim _{m \rightarrow \infty} \limsup _{n \rightarrow \infty} \operatorname{Pr}\left(\left|V_{T, k}-V_{T, k}^{m}\right|>\epsilon\right) \leq \lim _{m \rightarrow \infty} \frac{1}{m^{2}} \boldsymbol{\theta}^{\prime} \boldsymbol{R}_{k}^{\prime} \boldsymbol{\Lambda} \boldsymbol{R}_{k} \boldsymbol{\theta}=0
$$

The end result for the second term is $I I=2 \sum_{k=1}^{K} \sum_{t=1}^{T} V_{t T, k}(\boldsymbol{\theta}) \stackrel{o_{p}(1)}{=} 2 \sum_{k=1}^{K} \mu_{k}=$ $\sum_{k=1}^{K} f_{k}(0) \boldsymbol{\theta}^{\prime} \boldsymbol{R}_{k}^{\prime} \mathrm{E}\left(\boldsymbol{z}_{t-1} \boldsymbol{z}_{t-1}^{\prime} \mathbb{1}_{\left\{0<\boldsymbol{z}_{t-1}^{\prime} \boldsymbol{R}_{k} \boldsymbol{\theta}\right\}}\right) \boldsymbol{R}_{k} \boldsymbol{\theta}$. Using exactly the same steps, one can show that the term $I I I$ has the same limit apart from the indicator functions having the inequality signs reversed. Adding all contributions together, we conclude ${ }^{11}$

$$
Z_{T}^{*}(\boldsymbol{\theta}) \stackrel{d}{\longrightarrow} Z^{*}(\boldsymbol{\theta})=-\boldsymbol{\theta}^{\prime} \boldsymbol{X}+\boldsymbol{\theta}^{\prime} \boldsymbol{R}^{\prime}(\boldsymbol{\Xi} \otimes \boldsymbol{\Lambda}) \boldsymbol{R} \boldsymbol{\theta} .
$$

The quadratic form for $Z^{*}(\boldsymbol{\theta})$ is minimized at $\boldsymbol{\theta}_{\min }=\frac{1}{2}\left[\boldsymbol{R}^{\prime}(\boldsymbol{\Xi} \otimes \boldsymbol{\Lambda}) \boldsymbol{R}\right]^{-1} \boldsymbol{X}$ having the distribution $\mathrm{N}\left(\mathbf{0}, \frac{1}{4}\left[\boldsymbol{R}^{\prime}(\boldsymbol{\Xi} \otimes \boldsymbol{\Lambda}) \boldsymbol{R}\right]^{-1} \boldsymbol{R}^{\prime}(\mathrm{E}(\boldsymbol{\Psi}) \otimes \boldsymbol{\Lambda}) \boldsymbol{R}\left[\boldsymbol{R}^{\prime}(\boldsymbol{\Xi} \otimes \boldsymbol{\Lambda}) \boldsymbol{R}\right]^{-1}\right)$. The convexity lemma by Pollard (1991) states that the minimizer of $Z_{T}^{*}(\boldsymbol{\theta})$ (that is $\sqrt{T}\left(\widehat{\gamma}_{T}^{*}-\widehat{\gamma}_{T}\right)$ ) and the minimizer of $Z^{*}(\boldsymbol{\theta})$ (that is $\boldsymbol{\theta}_{\text {min }}$ ) share the same limiting distribution. This distribution coincides with the one of Theorem 4.1 and bootstrap consistency is established.

\footnotetext{
${ }^{11}$ We used the following identity to arrive at this expression:

$$
\sum_{k=1}^{K} f_{k}(0) \boldsymbol{\theta}^{\prime} \boldsymbol{R}_{k}^{\prime} \boldsymbol{\Lambda} \boldsymbol{R}_{k} \boldsymbol{\theta}=\boldsymbol{\theta}^{\prime}\left\{\left[\begin{array}{llll}
\boldsymbol{R}_{1}^{\prime} & \boldsymbol{R}_{2}^{\prime} & \cdots & \boldsymbol{R}_{K}^{\prime}
\end{array}\right]\left[\begin{array}{cccc}
f_{1}(0) \boldsymbol{\Lambda} & \mathbf{0} & \cdots & \mathbf{0} \\
\mathbf{O} & f_{2}(0) \boldsymbol{\Lambda} & \cdots & \mathbf{0} \\
\vdots & \vdots & \ddots & \vdots \\
\mathbf{0} & \mathbf{0} & \cdots & f_{K}(0) \boldsymbol{\Lambda}
\end{array}\right]\left[\begin{array}{c}
\boldsymbol{R}_{1} \\
\boldsymbol{R}_{2} \\
\vdots \\
\boldsymbol{R}_{K}
\end{array}\right]\right\} \boldsymbol{\theta}
$$
}




\section{A.2 Hypothesis Tests}

\section{Wald test}

The unrestricted estimator $\widehat{\boldsymbol{b}}_{T}^{U}$ is equal to $\widehat{\gamma}_{T}$ for $\boldsymbol{R}=$ and $\boldsymbol{r}=\mathbf{0}$. As such, Theorem 4.2 states that the bootstrap is correctly replicating the limiting distribution of $\sqrt{T}\left(\widehat{\boldsymbol{b}}_{T}^{U}-\boldsymbol{b}_{0}\right)$. The bootstrap consistency of $\widetilde{W}_{T}^{*}$ now follows from the delta method for the bootstrap. For instance, apply theorem 23.5 of van der Vaart (2000) with the map $\phi: \mathbb{R}^{K(K p+1)} \rightarrow \mathbb{R}$ defined by $\phi(\boldsymbol{x})=(\boldsymbol{C} \boldsymbol{x}-\boldsymbol{c})^{\prime}(\boldsymbol{C} \boldsymbol{x}-\boldsymbol{c})$.

\section{LM test}

Define:

$$
\tilde{\boldsymbol{\eta}}_{T}^{*}(\boldsymbol{\Delta})=-\frac{1}{\sqrt{T}} \sum_{t=1}^{T} \sum_{k=1}^{K} \boldsymbol{E}_{k}^{\prime} \boldsymbol{z}_{t-1} \operatorname{sgn}\left(u_{k t}^{*}-\boldsymbol{z}_{t-1}^{\prime} \boldsymbol{E}_{k} \boldsymbol{\Delta}\right)
$$

Applying the Huber result (Appendix 3.B and Fitzenberger (1997)) to the bootstrap gives

$$
\sup _{\|\boldsymbol{\Delta}\| \leq 1}\left|\tilde{\boldsymbol{\eta}}_{T}^{*}(\boldsymbol{\Delta})-\tilde{\boldsymbol{\eta}}_{T}^{*}(\mathbf{0})-\mathrm{E}^{*}\left[\tilde{\boldsymbol{\eta}}_{T}^{*}(\boldsymbol{\Delta})-\tilde{\boldsymbol{\eta}}_{T}^{*}(\mathbf{0})\right]\right|=o_{p}^{*}(1)
$$

where $E^{*}$ denotes the expectation under the fixed design residual bootstrap. The given expectation equals

$$
\mathrm{E}^{*}\left[\tilde{\boldsymbol{\eta}}_{T}^{*}(\boldsymbol{\Delta})-\tilde{\boldsymbol{\eta}}_{T}^{*}(\mathbf{0})\right]=\frac{1}{\sqrt{T}} \sum_{t=1}^{T} \sum_{k=1}^{K} \boldsymbol{E}_{k}^{\prime} \boldsymbol{z}_{t-1} \mathrm{E}^{*}\left[\operatorname{sgn}\left(u_{k t}^{*}-\boldsymbol{z}_{t-1}^{\prime} \boldsymbol{E}_{k} \Delta\right)-\operatorname{sgn}\left(u_{k t}^{*}\right)\right]
$$

We now develop the expectation in Equation (4.26) further using the equality $\operatorname{sgn}(a+b)-$ $\operatorname{sgn}(a)=2\left(\mathbb{1}_{\{-b \leq a<0\}} \mathbb{1}_{\{b>0\}}-\mathbb{1}_{\{0 \leq a<-b\}} \mathbb{1}_{\{b<0\}}\right)$, or

$$
\begin{aligned}
& \mathrm{E}^{*}\left[\operatorname{sgn}\left(u_{k t}^{*}-\boldsymbol{z}_{t-1}^{\prime} \boldsymbol{E}_{k} \boldsymbol{\Delta}\right)-\operatorname{sgn}\left(u_{k t}^{*}\right)\right] \\
& =2 \int_{\boldsymbol{z}_{t-1}^{\prime} \boldsymbol{E}_{k} \boldsymbol{\Delta}}^{0} d \widehat{F}_{T, k} \mathbb{1}_{\left\{\boldsymbol{z}_{t-1}^{\prime} \boldsymbol{E}_{k} \boldsymbol{\Delta}<0\right\}}-2 \int_{0}^{\boldsymbol{z}_{t-1}^{\prime} \boldsymbol{E}_{k} \boldsymbol{\Delta}} d \widehat{F}_{T, k} \mathbb{1}_{\left\{\boldsymbol{z}_{t-1}^{\prime} \boldsymbol{E}_{k} \boldsymbol{\Delta}>0\right\}} \\
& \stackrel{o_{p}(1)}{=} 2\left[F_{k}(0)-F_{k}\left(\boldsymbol{z}_{t-1}^{\prime} \boldsymbol{E}_{k} \boldsymbol{\Delta}\right)\right] \mathbb{1}_{\left\{\boldsymbol{z}_{t-1}^{\prime} \boldsymbol{E}_{k} \boldsymbol{\Delta}<0\right\}}-2\left[F_{k}\left(\boldsymbol{z}_{t-1}^{\prime} \boldsymbol{E}_{k} \boldsymbol{\Delta}\right)-F_{k}(0)\right] \mathbb{1}_{\left\{\boldsymbol{z}_{t-1}^{\prime} \boldsymbol{E}_{k} \boldsymbol{\Delta}>0\right\}} \\
& =2 \int_{\boldsymbol{z}_{t-1}^{\prime} \boldsymbol{E}_{k} \boldsymbol{\Delta}}^{0}\left(f_{k}(x)-f_{k}(0)\right) d x \mathbb{1}_{\left\{\boldsymbol{z}_{t-1}^{\prime} \boldsymbol{E}_{k} \boldsymbol{\Delta}<0\right\}}-2 \int_{0}^{\boldsymbol{z}_{t-1}^{\prime} \boldsymbol{E}_{k} \boldsymbol{\Delta}}\left(f_{k}(x)-f_{k}(0)\right) d x \mathbb{1}_{\left\{\boldsymbol{z}_{t-1}^{\prime} \boldsymbol{E}_{k} \boldsymbol{\Delta}>0\right\}} \\
& \quad-2 f_{k}(0) \boldsymbol{z}_{t-1}^{\prime} \boldsymbol{E}_{k} \boldsymbol{\Delta}=-2 f_{k}(0) \boldsymbol{z}_{t-1}^{\prime} \boldsymbol{E}_{k} \boldsymbol{\Delta}+O_{p}\left(\|\boldsymbol{\Delta}\|^{2}\right),
\end{aligned}
$$


where we have used $\sup _{x}\left(\widehat{F}_{T, k}(x)-F_{k}(x)\right)=o_{p}(1)$ in proceeding from the second to third line. In view of Assumption 4.2(e), the final line followed from

$$
\left|\int_{\boldsymbol{z}_{t-1}^{\prime} \boldsymbol{E}_{k} \boldsymbol{\Delta}}^{0}\left(f_{k}(x)-f_{k}(0)\right) d x\right| \leq L_{0} \int_{\boldsymbol{z}_{t-1}^{\prime} \boldsymbol{E}_{k} \boldsymbol{\Delta}}^{0}|x| d x=\frac{1}{2}\left(\boldsymbol{z}_{t-1}^{\prime} \boldsymbol{E}_{k} \boldsymbol{\Delta}\right)
$$

and a similar calculation for the second integral. On combining Equations (4.25)-(4.27), we have

$$
\mathrm{E}^{*}\left[\tilde{\boldsymbol{\eta}}_{T}^{*}(\boldsymbol{\Delta})-\tilde{\boldsymbol{\eta}}_{T}^{*}(\mathbf{0})\right]=-2\left(\frac{1}{T} \sum_{t=1}^{T} \sum_{k=1}^{K} f_{k}(0) \boldsymbol{E}_{k}^{\prime} \boldsymbol{z}_{t-1} \boldsymbol{z}_{t-1}^{\prime} \boldsymbol{E}_{k}\right) \sqrt{T} \boldsymbol{\Delta}+O_{p}\left(\sqrt{T}\|\boldsymbol{\Delta}\|^{2}\right)
$$

We subsequently take $\boldsymbol{\Delta}=\widehat{\boldsymbol{b}}_{T}^{R, *}-\widehat{\boldsymbol{b}}_{T}$. Note (1) that $\sqrt{T}\left(\widehat{\boldsymbol{b}}_{T}^{R, *}-\widehat{\boldsymbol{b}}_{T}\right)=O_{p}(1)$ under the null such that $\left\|\widehat{\boldsymbol{b}}_{T}^{R, *}-\widehat{\boldsymbol{b}}_{T}\right\| \leq 1$ holds with arbitrary high probability; (2) that the remainder term $O_{p}\left(\sqrt{T}\left\|\widehat{\boldsymbol{b}}_{T}^{R, *}-\widehat{\boldsymbol{b}}_{T}\right\|^{2}\right)$ vanishes and; (3) that $\tilde{\boldsymbol{\eta}}_{T}^{*}\left(\widehat{\boldsymbol{b}}_{T}^{R, *}-\widehat{\boldsymbol{b}}_{T}\right)=\boldsymbol{g}_{T}^{*}\left(\widehat{\boldsymbol{b}}_{T}^{R, *}\right)$. We conclude that

$$
\begin{aligned}
& \boldsymbol{g}_{T}^{*}\left(\widehat{\boldsymbol{b}}_{T}^{R, *}\right) \stackrel{o_{p}(1)}{=}-\frac{1}{\sqrt{T}} \sum_{t=1}^{T} \sum_{k=1}^{K} \boldsymbol{E}_{k}^{\prime} \boldsymbol{z}_{t-1} \operatorname{sgn}\left(u_{k t}^{*}\right)-2(\boldsymbol{\Xi} \otimes \boldsymbol{\Lambda}) \sqrt{T}\left(\widehat{\boldsymbol{b}}_{T}^{R, *}-\widehat{\boldsymbol{b}}_{T}\right) \\
& =\left[\begin{array}{ll}
-\boldsymbol{I}_{K(K p+1)} & -2(\boldsymbol{\Xi} \otimes \boldsymbol{\Lambda})
\end{array}\right]\left[\begin{array}{c}
\frac{1}{\sqrt{T}} \sum_{t=1}^{T} \sum_{k=1}^{K} \boldsymbol{E}_{k}^{\prime} \boldsymbol{z}_{t-1} \operatorname{sgn}\left(u_{k t}^{*}\right) \\
\sqrt{T}\left(\widehat{\boldsymbol{b}}_{T}^{R, *}-\widehat{\boldsymbol{b}}_{T}\right)
\end{array}\right] .
\end{aligned}
$$

Now compare this to the following statement from Section 3.A.5 (see page 99)

$$
\begin{aligned}
& \boldsymbol{g}_{T}\left(\widehat{b}_{T}^{R}\right) \stackrel{o_{p}(1)}{=} \boldsymbol{g}_{T}\left(\boldsymbol{b}_{0}\right)-\overline{\boldsymbol{H}}_{T} \sqrt{T}\left(\widehat{\boldsymbol{b}}_{T}^{R}-\boldsymbol{b}_{0}\right) \\
& \stackrel{o_{p}(1)}{=}-\frac{1}{\sqrt{T}} \sum_{t=1}^{T} \sum_{k=1}^{K} \boldsymbol{E}_{k}^{\prime} \boldsymbol{z}_{t-1} \operatorname{sgn}\left(u_{k t}\right)-2(\boldsymbol{\Xi} \otimes \boldsymbol{\Lambda}) \sqrt{T}\left(\widehat{\boldsymbol{b}}_{T}^{R}-\boldsymbol{b}_{0}\right) \\
&=\left[\begin{array}{ll}
-\boldsymbol{I}_{K(K p+1)} & -2(\boldsymbol{\Xi} \otimes \boldsymbol{\Lambda})
\end{array}\right]\left[\begin{array}{c}
\frac{1}{\sqrt{T}} \sum_{t=1}^{T} \sum_{k=1}^{K} \boldsymbol{E}_{k}^{\prime} \boldsymbol{z}_{t-1} \operatorname{sgn}\left(u_{k t}\right) \\
\sqrt{T}\left(\widehat{\boldsymbol{b}}_{T}^{R}-\boldsymbol{b}_{0}\right)
\end{array}\right]
\end{aligned}
$$

where we used the i.i.d. assumption on the innovations to evaluate the limit of $\overline{\boldsymbol{H}}_{T}$ while continuing to the second line. Equations (4.30) and (4.31) provide the bootstrap validity 
because an argument almost identical ${ }^{12}$ to the proof of Lemma 4.1 will show that

$$
d_{2}^{M}\left(\frac{1}{\sqrt{T}} \sum_{t=1}^{T} \sum_{k=1}^{K} \boldsymbol{E}_{k}^{\prime} z_{t-1} \operatorname{sgn}\left(u_{k t}^{*}\right), \frac{1}{\sqrt{T}} \sum_{t=1}^{T} \sum_{k=1}^{K} \boldsymbol{E}_{k}^{\prime} z_{t-1} \operatorname{sgn}\left(u_{k t}\right)\right)=o_{p}(1)
$$

so that their asymptotic distributions coincide. We already know from Theorem 4.2 that the same holds for the parameter estimates. This convergence also occurs jointly so the proof is complete. ${ }^{13}$

\footnotetext{
${ }^{12}$ The only difference is the use of $\boldsymbol{E}_{k}$ instead of $\boldsymbol{R}_{k}$ but this has virtually no influence on the proof because we only require these matrices to have a bounded norm.

${ }^{13}$ The asymptotic distribution of the bootstrapped parameter estimates was linked to the quantity $\frac{1}{\sqrt{T}} \sum_{t=1}^{T} \boldsymbol{\eta}_{t}^{*}=\frac{1}{\sqrt{T}} \sum_{t=1}^{T} \sum_{k=1}^{K} \boldsymbol{R}_{k}^{\prime} \boldsymbol{z}_{t-1} \operatorname{sgn}\left(u_{k t}^{*}\right)$ which converges jointly with $\sqrt{T} \sum_{t=1}^{T} \sum_{k=1}^{K} \boldsymbol{E}_{k}^{\prime} \boldsymbol{z}_{t-1} \operatorname{sgn}\left(u_{k t}^{*}\right)$.
} 


\section{B An MC Comparison with Unrestricted Residuals}

\begin{tabular}{|c|c|c|c|c|c|c|c|c|c|c|c|}
\hline & \multirow[b]{2}{*}{$\alpha$} & \multicolumn{3}{|c|}{$T=100$} & \multicolumn{3}{|c|}{$T=250$} & \multicolumn{3}{|c|}{$T=500$} & \multirow{11}{*}{ 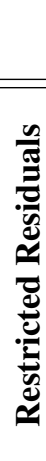 } \\
\hline & & Wald & $L M$ & $L R$ & Wald & $L M$ & $L R$ & Wald & $L M$ & $L R$ & \\
\hline \multirow{3}{*}{$\mathcal{N}(0,1)$} & 0 & 5.6 & 5.2 & 6.9 & 4.9 & 5.5 & 6.2 & 5.0 & 5.5 & 5.5 & \\
\hline & -0.1 & 14.7 & 8.9 & 14.7 & 28.9 & 16.5 & 25.7 & 45.1 & 22.8 & 38.8 & \\
\hline & -0.2 & 37.8 & 13.7 & 33.6 & 73.9 & 46.0 & 72.2 & 94.9 & 79.0 & 92.6 & \\
\hline \multirow{3}{*}{ Laplace } & 0 & 4.6 & 4.7 & 5.4 & 4.9 & 5.0 & 5.1 & 5.1 & 4.3 & 4.7 & \\
\hline & -0.1 & 18.8 & 11.4 & 23.3 & 48.7 & 29.2 & 46.9 & 80.2 & 58.5 & 77.4 & \\
\hline & -0.2 & 58.2 & 29.6 & 60.6 & 95.6 & 76.9 & 95.0 & 100.0 & 98.7 & 99.7 & \\
\hline \multirow{3}{*}{$\mathrm{t}(5)$} & 0 & 4.9 & 5.1 & 5.7 & 5.0 & 5.7 & 4.7 & 4.3 & 5.7 & 5.4 & \\
\hline & -0.1 & 17.6 & 9.0 & 17.6 & 33.1 & 20.6 & 32.8 & 60.0 & 36.1 & 56.2 & \\
\hline & -0.2 & 47.8 & 23.2 & 47.8 & 88.6 & 61.2 & 85.0 & 98.9 & 91.3 & 98.5 & \\
\hline \multirow{3}{*}{$\mathcal{N}(0,1)$} & 0 & 7.2 & 4.6 & 6.7 & 8.1 & 6.0 & 9.1 & 7.2 & 4.6 & 7.2 & \\
\hline & -0.1 & 19.0 & 7.7 & 17.8 & 30.0 & 15.0 & 24.9 & 45.8 & 24.7 & 41.6 & $\bar{z}$ \\
\hline & -0.2 & 45.5 & 15.6 & 42.8 & 75.5 & 41.9 & 69.9 & 95.2 & 81.3 & 92.9 & \\
\hline \multirow{3}{*}{ Laplace } & 0 & 6.8 & 4.2 & 6.6 & 5.8 & 4.3 & 5.4 & 7.2 & 5.5 & 6.4 & $\approx$ \\
\hline & -0.1 & 24.7 & 11.6 & 29.3 & 53.9 & 28.0 & 49.1 & 83.2 & 59.1 & 80.2 & ?्ల \\
\hline & -0.2 & 67.0 & 29.1 & 66.0 & 96.8 & 78.2 & 96.6 & 100.0 & 98.2 & 99.9 & 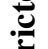 \\
\hline \multirow{3}{*}{$\mathrm{t}(5)$} & 0 & 9.2 & 3.6 & 8.5 & 7.0 & 4.1 & 6.7 & 7.6 & 5.2 & 7.1 & $\sigma_{0}^{2}$ \\
\hline & -0.1 & 20.0 & 9.4 & 20.9 & 38.3 & 19.4 & 35.2 & 62.2 & 33.9 & 55.1 & Ü \\
\hline & -0.2 & 53.6 & 22.0 & 50.9 & 88.0 & 58.5 & 85.0 & 99.0 & 92.5 & 99.0 & $\triangleright$ \\
\hline
\end{tabular}

Table 4.4: A comparison of the fixed design residual bootstrap with restricted and unrestricted residuals. The table shows the empirical size $(\alpha=0)$ and empirical power $(\alpha \neq 0)$ in percentages for three innovation distributions. The desired nominal size is $5 \%$. 


\section{C An MC Comparison with a Bootstrap Method with Recursive Design}

This appendix devotes attention to the recursive design bootstrap. The bootstrap algorithm is described in Figure 4.2. Tables 4.5 and 4.6 are the counterparts of Tables 4.1 and 4.2, meaning that they report the empirical size and power of the tests when based on a recursive design. The definitions of these test statistics are identical to those in the main text after replacing $\boldsymbol{y}_{t}$ and $\boldsymbol{z}_{t}$ by their corresponding quantities as generated by the recursion of Equation (4.33).

\section{Recursive Design Residual Bootstrap}

STEP 1: Given a presample $\boldsymbol{y}_{1}, \boldsymbol{y}_{2}, \ldots, \boldsymbol{y}_{p}$, compute the parameter estimates $\widehat{\boldsymbol{b}}_{T}$ and the test statistic of interest, say $T(\boldsymbol{Y})$.

STEP 2: Obtain the residual vectors

$$
\widehat{\boldsymbol{u}}_{t}=\boldsymbol{y}_{t}-\left(\boldsymbol{I}_{K} \otimes \boldsymbol{z}_{t-1}^{\prime}\right) \widehat{\boldsymbol{b}}_{T}, \quad \text { for } t=p+1, p+2, \ldots, T .
$$

STEP 3: As implied by both $\widehat{\boldsymbol{b}}_{T}=\boldsymbol{R} \widehat{\boldsymbol{\gamma}}_{T}+\boldsymbol{r}$ and $\widehat{\boldsymbol{b}}_{T}=\operatorname{vec}\left(\left(\widehat{\boldsymbol{\nu}}_{T}, \widehat{\boldsymbol{A}}_{1, T}, \ldots, \widehat{\boldsymbol{A}}_{p, T}\right)^{\prime}\right)$, construct bootstrap samples recursively as

$$
\boldsymbol{y}_{t}^{\dagger}=\widehat{\boldsymbol{\nu}}_{T}+\widehat{\boldsymbol{A}}_{1, T} \boldsymbol{y}_{t-1}^{\dagger}+\ldots+\widehat{\boldsymbol{A}}_{p, T} \boldsymbol{y}_{t-p}^{\dagger}+\boldsymbol{u}_{t}^{\dagger}
$$

while initializing from the presample $\boldsymbol{y}_{1}, \boldsymbol{y}_{2}, \ldots, \boldsymbol{y}_{p}$ and resampling $\boldsymbol{u}_{t}^{\dagger}$ with replacement from $\left\{\widehat{\boldsymbol{u}}_{p+1}, \widehat{\boldsymbol{u}}_{p+2}, \ldots, \widehat{\boldsymbol{u}}_{T}\right\}$.

STEP 4: Repeat the previous step $B$ times and select the bootstrap critical value, say $c_{\alpha, B}^{*}$, as the $(1-\alpha)$-quantile of the ordered bootstrap test statistics $T\left(\boldsymbol{Y}^{*}\right)$. Reject the null hypothesis if $T(\boldsymbol{Y})>c_{\alpha, B}^{*}$.

Figure 4.2: The bootstrap algorithm for the recursive design residual bootstrap. Note that the vector $\boldsymbol{y}_{t}^{\dagger}$ is computed using $\boldsymbol{z}_{t-1}^{\dagger}$. 


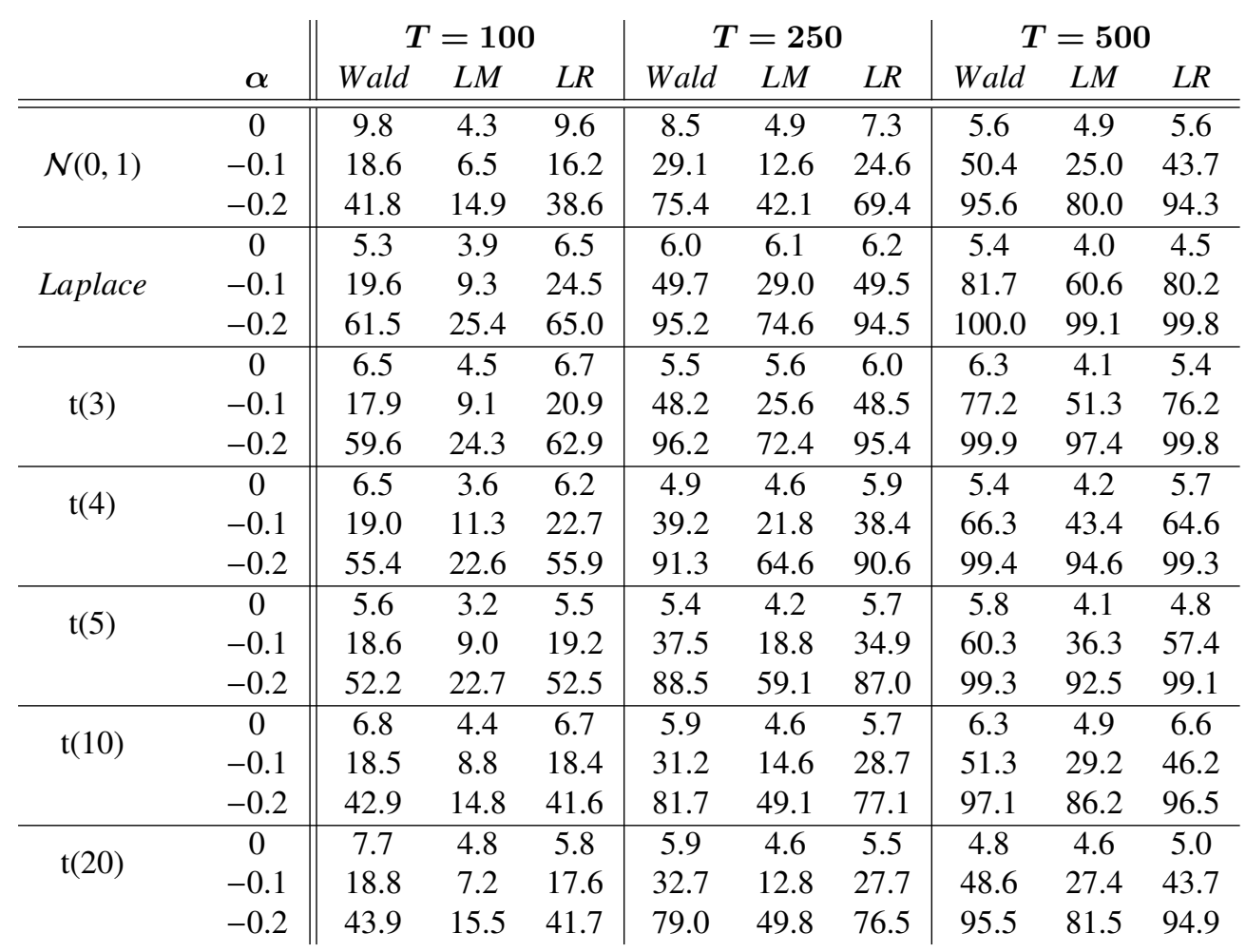

Table 4.5: The empirical size and power (in percentages) of the three bootstrapped test statistics of Equation (4.5) for various innovation distributions and sample sizes. The desired nominal size is $5 \%$. The data was generated according to the model specified by Equation (4.6). Results are based on the recursive design residual bootstrap. 


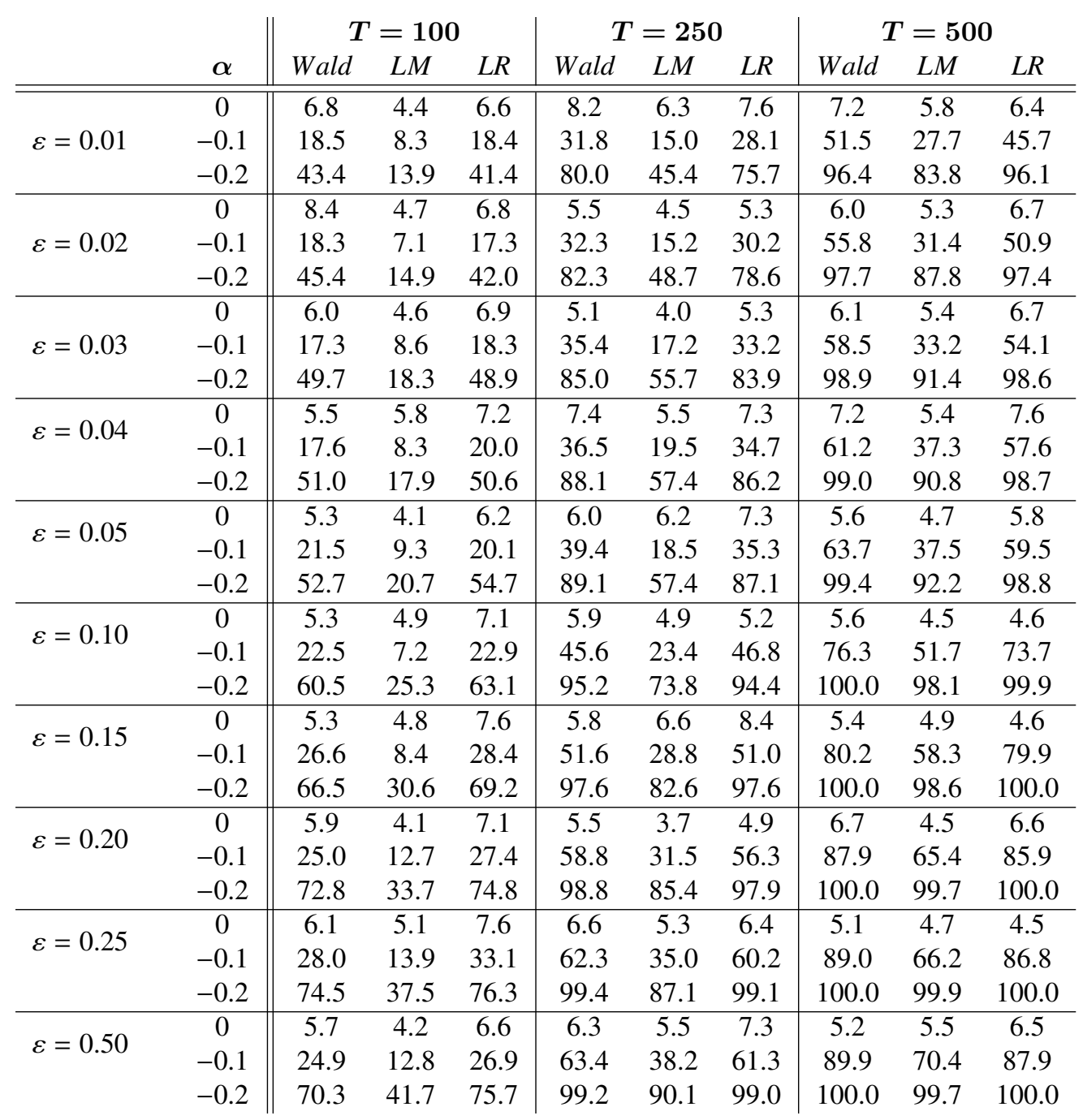

Table 4.6: The empirical size and power (in percentages) of the three bootstrapped test statistics of Equation (4.5) for a varying percentage of outliers in the mixture distribution and three sample sizes. The desired nominal size is 5\%. See Equation (4.6) for the data generating process. Results are based on the recursive design residual bootstrap. 


\begin{tabular}{|c|c|c|c|c|c|c|c|c|c|c|c|}
\hline & \multirow[b]{2}{*}{$\alpha$} & \multicolumn{3}{|c|}{$T=100$} & \multicolumn{3}{|c|}{$T=250$} & \multicolumn{3}{|c|}{$T=500$} & \\
\hline & & Wald & $L M$ & $L R$ & Wald & $L M$ & $L R$ & Wald & $L M$ & $L R$ & \\
\hline \multirow{3}{*}{$\mathcal{N}(0,1)$} & 0 & 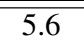 & 5.2 & 6.9 & 4.9 & 5.5 & 6.2 & 5.0 & 5.5 & 5.5 & \multirow{15}{*}{ 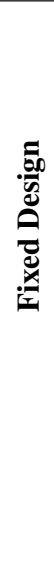 } \\
\hline & -0.1 & 14.7 & 8.9 & 14.7 & 28.9 & 16.5 & 25.7 & 45.1 & 22.8 & 38.8 & \\
\hline & -0.2 & 37.8 & 13.7 & 33.6 & 73.9 & 46.0 & 72.2 & 94.9 & 79.0 & 92.6 & \\
\hline \multirow{3}{*}{ Laplace } & 0 & 4.6 & 4.7 & 5.4 & 4.9 & 5.0 & 5.1 & 5.1 & 4.3 & 4.7 & \\
\hline & -0.1 & 18.8 & 11.4 & 23.3 & 48.7 & 29.2 & 46.9 & 80.2 & 58.5 & 77.4 & \\
\hline & -0.2 & 58.2 & 29.6 & 60.6 & 95.6 & 76.9 & 95.0 & 100.0 & 98.7 & 99.7 & \\
\hline \multirow{3}{*}{$\mathrm{t}(3)$} & 0 & 4.6 & 5.4 & 4.7 & 5.7 & 4.9 & 5.7 & 5.3 & 5.3 & 5.7 & \\
\hline & -0.1 & 20.6 & 11.5 & 23.3 & 49.5 & 25.7 & 46.1 & 78.8 & 49.8 & 75.0 & \\
\hline & -0.2 & 61.4 & 27.3 & 65.3 & 95.5 & 70.3 & 94.0 & 100.0 & 96.7 & 100.0 & \\
\hline \multirow{3}{*}{$\mathrm{t}(5)$} & 0 & 4.9 & 5.1 & 5.7 & 5.0 & 5.7 & 4.7 & 4.3 & 5.7 & 5.4 & \\
\hline & -0.1 & 17.6 & 9.0 & 17.6 & 33.1 & 20.6 & 32.8 & 60.0 & 36.1 & 56.2 & \\
\hline & -0.2 & 47.8 & 23.2 & 47.8 & 88.6 & 61.2 & 85.0 & 98.9 & 91.3 & 98.5 & \\
\hline \multirow{3}{*}{$\mathrm{t}(20)$} & 0 & 3.9 & 4.3 & 4.9 & 6.0 & 5.5 & 6.3 & 5.2 & 5.6 & 6.3 & \\
\hline & -0.1 & 14.0 & 8.1 & 14.2 & 27.2 & 14.6 & 24.1 & 50.3 & 28.4 & 43.2 & \\
\hline & -0.2 & 40.8 & 16.3 & 39.5 & 76.1 & 47.7 & 73.6 & 96.6 & 81.4 & 95.0 & \\
\hline \multirow{3}{*}{$\mathcal{N}(0,1)$} & 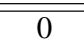 & 9.8 & 4.3 & 9.6 & 8.5 & 4.9 & 7.3 & 5.6 & 4.9 & 5.6 & \multirow{15}{*}{ 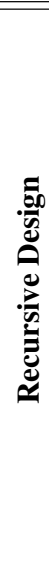 } \\
\hline & -0.1 & 18.6 & 6.5 & 16.2 & 29.1 & 12.6 & 24.6 & 50.4 & 25.0 & 43.7 & \\
\hline & -0.2 & 41.8 & 14.9 & 38.6 & 75.4 & 42.1 & 69.4 & 95.6 & 80.0 & 94.3 & \\
\hline \multirow{3}{*}{ Laplace } & 0 & 5.3 & 3.9 & 6.5 & 6.0 & 6.1 & 6.2 & 5.4 & 4.0 & 4.5 & \\
\hline & -0.1 & 19.6 & 9.3 & 24.5 & 49.7 & 29.0 & 49.5 & 81.7 & 60.6 & 80.2 & \\
\hline & -0.2 & 61.5 & 25.4 & 65.0 & 95.2 & 74.6 & 94.5 & 100.0 & 99.1 & 99.8 & \\
\hline \multirow{3}{*}{$\mathrm{t}(3)$} & 0 & 6.5 & 4.5 & 6.7 & 5.5 & 5.6 & 6.0 & 6.3 & 4.1 & 5.4 & \\
\hline & -0.1 & 17.9 & 9.1 & 20.9 & 48.2 & 25.6 & 48.5 & 77.2 & 51.3 & 76.2 & \\
\hline & -0.2 & 59.6 & 24.3 & 62.9 & 96.2 & 72.4 & 95.4 & 99.9 & 97.4 & 99.8 & \\
\hline \multirow{3}{*}{$\mathrm{t}(5)$} & 0 & 5.6 & 3.2 & 5.5 & 5.4 & 4.2 & 5.7 & 5.8 & 4.1 & 4.8 & \\
\hline & -0.1 & 18.6 & 9.0 & 19.2 & 37.5 & 18.8 & 34.9 & 60.3 & 36.3 & 57.4 & \\
\hline & -0.2 & 52.2 & 22.7 & 52.5 & 88.5 & 59.1 & 87.0 & 99.3 & 92.5 & 99.1 & \\
\hline \multirow{3}{*}{$\mathrm{t}(20)$} & 0 & 7.7 & 4.8 & 5.8 & 5.9 & 4.6 & 5.5 & 4.8 & 4.6 & 5.0 & \\
\hline & -0.1 & 18.8 & 7.2 & 17.6 & 32.7 & 12.8 & 27.7 & 48.6 & 27.4 & 43.7 & \\
\hline & -0.2 & 43.9 & 15.5 & 41.7 & 79.0 & 49.8 & 76.5 & 95.5 & 81.5 & 94.9 & \\
\hline
\end{tabular}

Table 4.7: Simulation results on the empirical size $(\alpha=0)$ and power $(\alpha \neq 0)$ of tests based on the two bootstrap algorithms: the fixed design residual bootstrap (Figure 4.1) and the recursive design residual bootstrap (Figure 4.2). The desired nominal size is $5 \%$. 


\section{D Simulation Results on Median Resampling}

Figure 4.3 shows the algorithm that uses residual resampling to estimate the densities at zero. The Wald and Score test were carried out in two ways. We either directly compared the values of these test statistics to the critical values of a chi-squared distribution (as suggested by Theorems 3.4 and 3.5) or used a double bootstrap procedure to obtain critical values from the bootstrap distribution.

Simulation results are reported in Table 4.8. The rejection frequencies at $\alpha=0$ and $\alpha \neq 0$ report empirical size and power, respectively. We took $B_{1}=B_{2}=499$ and mention that the computational costs of the single and double bootstrap are comparable because both the resampling and the median calculations of the residuals are fast. Table 4.8 indicates that single bootstrap Wald statistic can be severely oversized although the size moves towards the nominal size as the sample size increases. All other tests have a size comparable to the nominal level. 


\section{Median Resampling Bootstrap}

STEP 1: Given a presample $\boldsymbol{y}_{1}, \boldsymbol{y}_{2}, \ldots, \boldsymbol{y}_{p}$, compute the parameter estimates $\widehat{\boldsymbol{b}}_{T}$ and the test statistic of interest, say $T(Y)$.

STEP 2: Obtain the residual vectors

$$
\widehat{\boldsymbol{u}}_{t}=\boldsymbol{y}_{t}-\left(\boldsymbol{I}_{K} \otimes z_{t-1}^{\prime}\right) \widehat{\boldsymbol{b}}_{T}, \quad \text { for } t=p+1, p+2, \ldots, T .
$$

Compute the median $\widehat{q}_{k, T}$ of the residuals in the $k^{\prime}$ th cross-section.

STEP 3: For every $k=1,2 \ldots, K$, generate a bootstrap sample of the residuals by drawing with replacement from $\left\{\widehat{u}_{k, p+1}, \widehat{u}_{k, p+2}, \ldots, \widehat{u}_{k, T}\right\}$. Repeat this step $B_{2}$ times while storing the medians of the bootstrap sample, say $\widehat{q}_{k, T}^{*, b}$ for $b=1,2, \ldots, B_{2}$. Use

$$
\widetilde{f_{k}(0)}=\frac{1}{2 \sqrt{\frac{1}{B_{2}} \sum_{b=1}^{B_{2}}\left[\sqrt{T}\left(\widehat{q}_{k, T}^{*, b}-\widehat{q}_{k, T}\right)\right]^{2}}}
$$

as an estimate for $f_{k}(0)$.

STEP 4: Compute the Wald statistic

$$
\widetilde{W}_{T}=T\left(\boldsymbol{C} \widehat{\boldsymbol{b}}_{T}^{U}-\boldsymbol{c}\right)^{\prime}\left[\frac{1}{4} \boldsymbol{C}\left(\widetilde{\boldsymbol{\Xi}}^{-1} \widehat{\boldsymbol{\Psi}}^{-1} \otimes \widehat{\boldsymbol{\Lambda}}^{-1}\right) \boldsymbol{C}^{\prime}\right]^{-1}\left(\boldsymbol{C} \widehat{\boldsymbol{b}}_{T}^{U}-\boldsymbol{c}\right)
$$

and/or the LM statistic

$\widetilde{S}_{T}=\boldsymbol{g}_{T}\left(\widehat{\boldsymbol{b}}_{T}^{R}\right)^{\prime}\left[\widetilde{\Xi} \otimes \widehat{\boldsymbol{\Lambda}}^{-1} \boldsymbol{C}^{\prime}\left[\boldsymbol{C}\left(\widetilde{\Xi}^{-1} \widehat{\boldsymbol{\Psi}}^{-1} \otimes \widehat{\boldsymbol{\Lambda}}^{-1}\right) \boldsymbol{C}^{\prime}\right]^{-1} \boldsymbol{C}[\widetilde{\Xi} \otimes \widehat{\boldsymbol{\Lambda}}]^{-1} \boldsymbol{g}_{T}\left(\widehat{\boldsymbol{b}}_{T}^{R}\right)\right.$,

with $\widetilde{\Xi}=\operatorname{diag}\left(\widetilde{f_{1}(0)}, \widetilde{f_{2}(0)}, \ldots, \widetilde{f_{K}(0)}\right), \widehat{\boldsymbol{\Lambda}}=\frac{1}{T} \sum_{t=1}^{T} \boldsymbol{z}_{t-1} \boldsymbol{z}_{t-1}^{\prime}$ and $\widehat{\boldsymbol{\Psi}}$ having $(i, j)$ 'th element $\widehat{\boldsymbol{\Psi}}_{i j}=\frac{1}{T} \sum_{t=1}^{T} \operatorname{sgn}\left(\hat{u}_{i t}\right) \operatorname{sgn}\left(\hat{u}_{j t}\right)$.

STEP 5: For a test of size $\alpha$, proceed with either option (A) for the single bootstrap or option (B) for the double bootstrap.

(A) Reject the null hypothesis if the test statistic exceeds $\chi_{J, 1-\alpha}^{2}$, i.e. the $(1-\alpha)$ quantile of a chi-squared distributed random variable with $J$ degrees of freedom.

(B) Generate $B_{1}$ bootstrap samples as in step 3 of Figure 4.1 and repeat the steps 2-4 above on every bootstrap sample. Select the bootstrap critical value, say $\widetilde{c}_{\alpha, B_{1}}^{*}$, as the $(1-\alpha)$-quantile of the ordered bootstrap Wald and/or LM statistic. Reject the null hypothesis if the test statistic exceeds $\widetilde{c}_{\alpha, B_{1}}^{*}$.

Figure 4.3: The bootstrap algorithm for density estimation by residual resampling. 


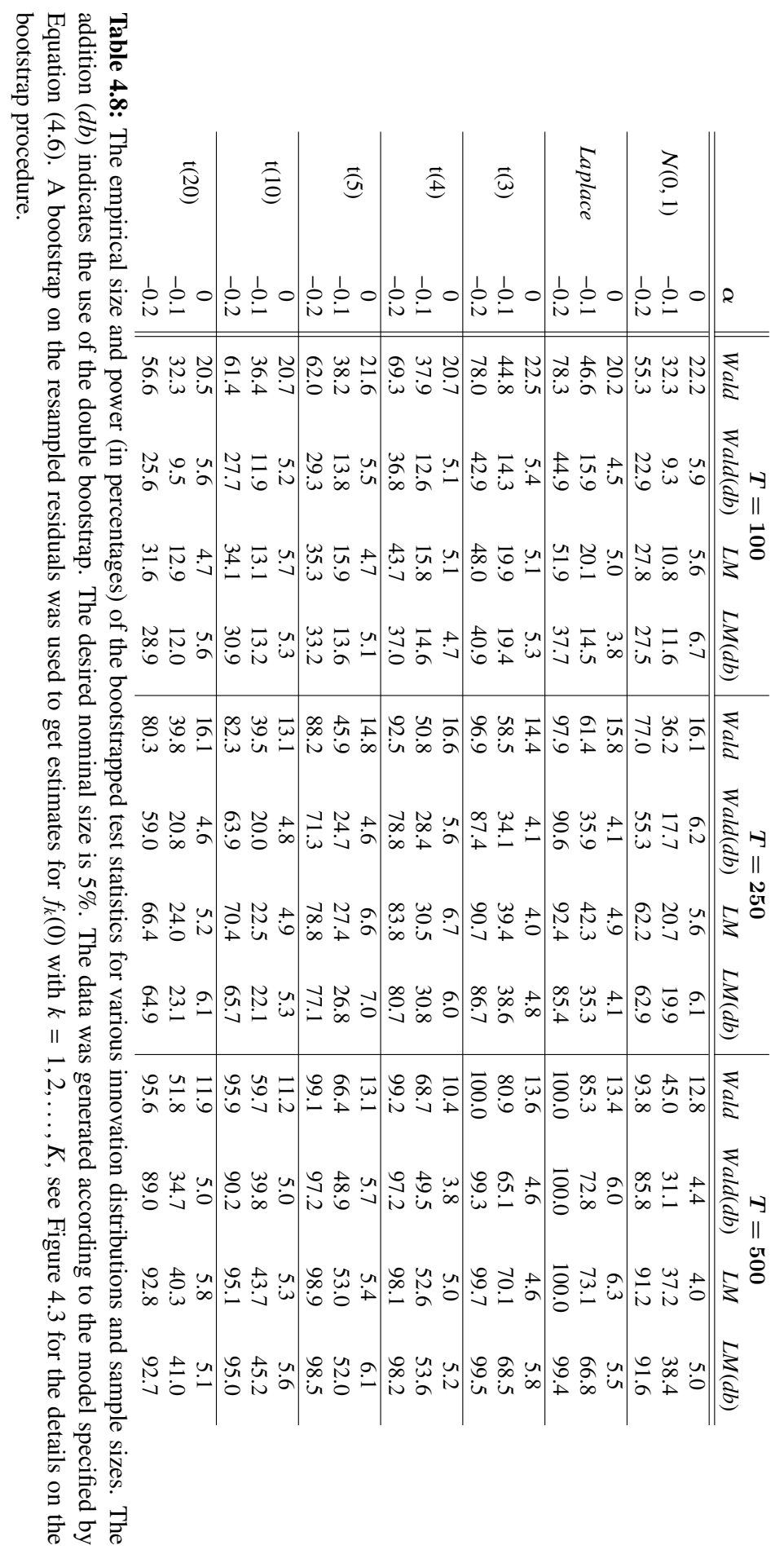




\section{Chapter 5}

\section{Conclusion}

"Reasoning draws a conclusion, but does not make the conclusion certain, unless the mind discovers it by the path of experience." - Roger Bacon (c. 1220-c. 1292)

This chapter concludes this thesis as a whole. As such, I have decided to divide this conclusion into two parts. The first part quickly reviews the chapter specific conclusions that you have already encountered at the end of each of the core chapters. I will be concise because extended versions can be found on the pages 39, 71 and 126. More general conclusions, i.e. conclusions with a wider scope, follow afterwards.

\subsection{Concluding Remarks on the Individual Chapters}

Chapter 2 studied the Focused Information Criterion (FIC) and plug-in average for vector autoregressions under local-to-zero misspecification. These procedures aim to minimize the asymptotic mean-squared error of the researcher's quantity of interest. We would have expected these tailored methods to outperform the alternatives that are based on global model fit (e.g. AIC, smoothed AIC and Jackknife model averaging). However, this anticipation was not confirmed by simulations, not even at a sample size as large as $T=1000$. We explained this finding by the inability to consistently estimate the localto-zero parameters. This claim was supported by the good performance of the infeasible versions of the FIC and plug-in average that make use of the true local-to-zero parameters. Personally, I conclude from Chapter 2 that there is a need to consider alternative ways to estimate the local-to-zero parameters. 
Statistical inference on VAR models with Least Absolute Deviations (LAD) was put forward in Chapter 3. We discussed: (1) the asymptotic distribution of the LAD estimator; (2) hypothesis tests on linear restrictions; and (3) information criteria based on the LAD objective function. For stationary VAR models, these three contributions give the LAD estimator a generality that is comparable to the least squares estimator (see e.g. chapters 1-5 of Lütkepohl (2005)). Simulations showed that the LAD estimator enjoys better performance in terms of MSE and testing power under departures from normality.

The validity of the fixed design residual bootstrap for the LAD estimator of the VAR coefficients was established in Chapter 4. The results in that chapter allowed us to: (1) construct percentile confidence intervals; and (2) test linear hypotheses with Wald and Lagrange Multiplier types of test statistics. These bootstrap resampling methods are easy to implement and could therefore be considered more user-friendly than density estimation methods. The simulation results recommended the use of the Wald type of test statistic in combination with a fixed design.

\subsection{General Conclusions}

The contents of Chapter 2 and 3 might raise the questions whether these topics can be combined. That is, can we define a Focused Information Criterion (FIC) that adopts LAD estimation? Without giving a formal proof, I will argue that this is indeed possible. An inspection of the mathematical structure of the derivations of Chapter 2 reveals that the most difficult step is the asymptotic normality of the estimator when some parameters are local-to-zero. This obstacle can probably be overcome by extending some of the univariate results by Weiss (1991). Subsequent steps are standard. The first order delta method would provide the asymptotic MSE for the estimators and weighted combinations thereof. These AMSE expressions would immediately define the FIC and plug-in averages based on least absolute deviations. One could even think along the lines of Chen et al. (2016) and let both the LAD and the OLS estimators enter the plug-in averages. However, we would still have inconsistent estimates for the local-to-zero parameters and the related suboptimal performance.

After having seen the asymptotics of the LAD estimator in Chapter 3, it is also sensible to return to the introduction. Specifically, let me comment in more detail on the (possible) benefit of having both LAD and OLS as estimation methods. In view of model uncertainty, it should be intuitively clear that similar estimates provide support for the chosen model whereas large difference cast doubt on the model specification. This reasoning 
in terms of differences between parameter estimates naturally hints towards a Hausman type of test (e.g. Hausman (1978) and Holly (1982)). Let us write $\widehat{\boldsymbol{\theta}}_{L A D}$ and $\widehat{\boldsymbol{\theta}}_{O L S}$ for the least absolute deviations and least squares estimator, respectively. The behavior of the difference $\widehat{\boldsymbol{\theta}}_{L A D}-\widehat{\boldsymbol{\theta}}_{O L S}$ is of current interest. Under a suitable set of assumptions ${ }^{1}$ both $\widehat{\boldsymbol{\theta}}_{L A D}$ and $\widehat{\boldsymbol{\theta}}_{O L S}$ would converge in probability to the true parameter vector, say $\boldsymbol{\theta}_{0}$. We will view this as 'correct specification'. The canonical quadratic form in $\widehat{\boldsymbol{\theta}}_{L A D}-\widehat{\boldsymbol{\theta}}_{O L S}$ would be asymptotically chi-squared distributed under the null of correct specification. It remains an open (and interesting) question to see which type(s) of misspecification such a test can detect. Any form of misspecification can only be detected if $\widehat{\boldsymbol{\theta}}_{L A D}$ and $\widehat{\boldsymbol{\theta}}_{O L S}$ converge to different limits (or equivalently converge to different pseudo-true parameters). At first sight, this does not seem an easy question to answer. ${ }^{2}$

My final comments will be on the combination of bootstrap and LAD and/or quantile estimation. As a first observation it appears to me as if comparison papers like Buchinsky (1995) are missing in the latest time series applications related to LAD. We have seen that the LAD framework requires some method to deal with the density at zero of the innovations. In Chapters 3 and 4, we have used kernel density estimation and bootstrap methods, respectively. In our simulation setting, the bootstrap method based on the Wald type of test was preferred over kernel density estimation (see Chapter 4, especially Table 4.3). Can we find further support for this claim in settings like the quantile unit root testing discussed in Koenker and Xiao (2004) and the quantile single-equation cointegration estimation by Xiao (2009) $?^{3}$ Secondly, it was already mentioned in the conclusion of Chapter 4 that it would be interesting to see whether a Wild bootstrap method can be used to conduct valid bootstrap inference under (conditional) heteroskedasticity of the innovations of the VAR model.

\footnotetext{
${ }^{1}$ This 'suitable' set of assumptions would be the union of the assumptions that are needed for consistency and asymptotic normality of the individual estimators. As an example, we would need innovations that have both a mean and a median of zero and the moment conditions of the OLS estimator because these are strongest.

${ }^{2}$ Pseudo-true parameters are easily derived for the OLS estimator because it is available in closed-form. The situation is different for the LAD estimator. We refer to the proof of 'Identifiable uniqueness' on page 88 . In this proof we started from the assumption that the objective function was minimized at the true parameters and proved this conjecture to be true. Under misspecification it does not appear to be straightforward to come up with such a initial starting assumption. A reference on the behavior of the LAD/quantile estimator under misspecification is Angrist et al. (2006).

${ }^{3} \mathrm{~A}$ third method that remained popular is in the literature is based on order statistics. What happens if we would also compare to this method?
} 



\section{Bibliography}

Abadir, K. and J. Magnus (2002). Notation in econometrics: a proposal for a standard. The Econometrics Journal 5, 76-90.

Akaike, H. (1998). Information theory and an extension of the maximum likelihood principle. In Selected Papers of Hirotugu Akaike, pp. 199-213. Springer.

Alqallaf, F., S. V. Aelst, V. J. Yohai, and R. H. Zamar (2009). Propagation of outliers in multivariate data. The Annals of Statistics 37, 311-331.

Andrews, D. W. K. (1987). Consistency in nonlinear econometric models: A generic uniform law of large numbers. Econometrica: Journal of the Econometric Society 55, 1465-1471.

Andrews, D. W. K. (1988). Laws of large numbers for dependent non-identically distributed random variables. Econometric Theory 4, 458-467.

Angrist, J., V. Chernozhukov, and I. Fernández-Val (2006). Quantile regression under misspecification, with an application to the US wage structure. Econometrica: Journal of the Econometric Society 74, 539-563.

Arcones, M. A. (1996). The Bahadur-Kiefer representation of $L_{p}$ regression estimators. Econometric Theory 12, 257-283.

Bańbura, M., D. Giannone, and L. Reichlin (2010). Large Bayesian vector auto regressions. Journal of Applied Econometrics 25, 71-92.

Barrodale, I. and F. D. K. Roberts (1978). An efficient algorithm for discrete L_1 linear approximation with linear constraints. SIAM Journal on Numerical Analysis 15, 603611. 
Bartels, R. H., A. R. Conn, and J. W. Sinclair (1978). Minimization techniques for piecewise differentiable functions: The $\mathrm{L}_{-} 1$ solution to an overdetermined linear system. SIAM Journal on Numerical Analysis 15, 224-241.

Bartle, R. G. (1966). The Elements of Integration and Lebesgue Measure. John Wiley \& Sons.

Bates, J. M. and C. W. J. Granger (1969). The combination of forecasts. Journal of the Operational Research Society 20, 451-468.

Benkwitz, A., M. H. Neumann, and H. Lütkepohl (2000). Problems related to confidence intervals for impulse responses of autoregressive processes. Econometric Reviews 19, 69-103.

Beran, R. (1987). Prepivoting to reduce level error of confidence sets. Biometrika 74, 457-468.

Beran, R. (1988). Prepivoting test statistics: a bootstrap view of asymptotic refinements. Journal of the American Statistical Association 83, 687-697.

Berk, K. N. (1974). Consistent autoregressive spectral estimates. The Annals of Statistics 2, 489-502.

Bernanke, B. S., J. Boivin, and P. Eliasz (2005). Measuring the effects of monetary policy: a factor-augmented vector autoregressive (FAVAR) approach. The Quarterly Journal of Economics 120, 387-422.

Bickel, P. J. and D. A. Freedman (1981). Some asymptotic theory for the bootstrap. The Annals of Statistics 9, 1196-1217.

Bierens, H. J. (1981). Robust Methods and Asymptotic Theory in Nonlinear Econometrics. New York: Springer-Verlag.

Bloomfield, P. and W. Steiger (1980). Least absolute deviations curve-fitting. SIAM Journal on Scientific and Statistical Computing 1, 290-301.

Bloomfield, P. and W. Steiger (1983). Least Absolute Deviations: Theory, Applications, and Algorithms. Birkhauser.

Boldin, M. V. (1983). Estimation of the distribution of noise in an autoregression scheme. Theory of Probability $\mathcal{E}$ Its Applications 27, 866-871. 
Botev, Z. I., J. F. Grotowski, and D. P. Kroese (2010). Kernel density estimation via diffusion. The Annals of Statistics 38, 2916-2957.

Breusch, T. S. (1978). Testing for autocorrelation in dynamic linear models. Australian Economic Papers 17, 334-355.

Brillinger, D. R. (2001). Time Series: Data Analysis and Theory. Society for Industrial and Applied Mathematics (SIAM).

Bruder, S. and M. Wolf (2017). Balanced bootstrap joint confidence bands for structural impulse response functions. Working Paper.

Brüggemann, R., C. Jentsch, and C. Trenkler (2016). Inference in VARs with conditional heteroskedasticity of unknown form. Journal of Econometrics 191, 69-85.

Buchinsky, M. (1995). Estimating the asymptotic covariance matrix for quantile regression models: a monte carlo study. Journal of Econometrics 68, 303-338.

Burnham, K. P. and D. R. Anderson (2002). Model Selection and Multimodel Inference: A Practical Information-Theoretic Approach. Springer.

Charkhi, A., G. Claeskens, and B. E. Hansen (2016). Minimum mean squared error model averaging in likelihood models. Statistica Sinica 26, 809-840.

Chatfield, C. (1995). Model uncertainty, data mining and statistical inference. Journal of the Royal Statistical Society. Series A. 158, 419-466.

Chen, X., D. T. Jacho-Chávez, and O. Linton (2016). Averaging of an increasing number of moment condition estimators. Econometric Theory 32, 30-70.

Chernozhukov, V. and I. Fernández-Val (2005). Subsampling inference on quantile regression processes. Sankhyā: The Indian Journal of Statistics 67, 253-276.

Cho, J. S., T.-H. Kim, and Y. Shin (2015). Quantile cointegration in the autoregressive distributed-lag modeling framework. Journal of Econometrics 188, 281-300.

Claeskens, G., C. Croux, and J. van Kerckhoven (2007). Prediction-focused model selection for autoregressive models. Australian $\mathcal{E}$ New Zealand Journal of Statistics 49, 359-379.

Claeskens, G. and N. L. Hjort (2003). The focused information criterion. Journal of the American Statistical Association 98, 900-916. 
Claeskens, G. and N. L. Hjort (2008). Model Selection and Model Averaging. Cambridge University Press: Cambridge.

Cochrane, J. H. (2007). The dog that did not bark: A defense of return predictability. The Review of Financial Studies 21, 1533-1575.

Cramér, H. (1946). Mathematical Methods of Statistics. Princeton University Press.

Daniels, H. E. (1961). The asymptotic efficiency of a maximum likelihood estimator. In Fourth Berkeley Symposium on Mathematical Statistics and Probability, Volume 1, pp. $151-163$.

Davidson, J. (1994). Stochastic Limit Theory: An Introduction for Econometricians. Oxford University Press, UK.

Davidson, R. and E. Flachaire (2008). The wild bootstrap, tamed at last. Journal of Econometrics 146, 162-169.

Diaconis, P. and B. Efron (1983). Computer intensive methods in statistics. Technical Report No. 83, Stanford University.

DiTraglia, F. J. (2016). Using invalid instruments on purpose: Focused moment selection and averaging for GMM. Journal of Econometrics 195, 187-208.

Edgeworth, F. Y. (1887). XXXIII. The choice of means. The London, Edinburgh, and Dublin Philosophical Magazine and Journal of Science 24, 268-271.

Engle, R. F. (1984). Wald, likelihood ratio, and lagrange multiplier tests in econometrics. Handbook of Econometrics 2, 775-826.

Engle, R. F. and C. W. J. Granger (1987). Co-integration and error correction: Representation, estimation, and testing. Econometrica: Journal of the Econometric Society 55, 251-276.

Engle, R. F. and S. Manganelli (2004). CAViaR: Conditional autoregressive value at risk by regression quantiles. Journal of Business $\mathcal{E}$ Economic Statistics 22, 367-381.

Feng, X., X. He, and J. Hu (2011). Wild bootstrap for quantile regression. Biometrika 98, 995-999.

Fitzenberger, B. (1997). The moving blocks bootstrap and robust inference for linear least squares and quantile regressions. Journal of Econometrics 82, 235-287. 
Freedman, D. A. (1981). Bootstrapping regression models. The Annals of Statistics 9, 1218-1228.

Fujiwara, M. (1916). Ueber die obere Schranke des absoluten Betrages der Wurzeln einer algebraischen Gleichung. Tohoku Mathematica Journal 10, 161-171.

Fuller, W. A. (1996). Introduction to Statistical Time Series. John Wiley \& Sons.

Gallant, A. R. and H. White (1988). A Unified Theory of Estimation and Inference for Nonlinear Dynamic Models. Blackwell.

Garratt, A., G. Koop, E. Mise, and S. P. Vahey (2009). Real-time prediction with UK monetary aggregates in the presence of model uncertainty. Journal of Business $\mathcal{E}$ Economic Statistics 27, 480-491.

Ghysels, E. (2016). Macroeconomics and the reality of mixed frequency data. Journal of Econometrics 193, 294-314.

Giacomini, R. (2013). The relationship between DSGE and VAR models. In VAR Models in Macroeconomics-New Developments and Applications: Essays in Honor of Christopher A. Sims, pp. 1-25. Emerald Group Publishing Limited.

Giannone, D., M. Lenza, and G. E. Primiceri (2015). Prior selection for vector autoregressions. Review of Economics and Statistics 97, 436-451.

Godfrey, L. G. (1978). Testing against general autoregressive and moving average error models when the regressors include lagged dependent variables. Econometrica: Journal of the Econometric Society 46, 1293-1301.

Goebel, R., A. Roebroeck, D.-S. Kim, and E. Formisano (2003). Investigating directed cortical interactions in time-resolved fMRI data using vector autoregressive modeling and Granger causality mapping. Magnetic Resonance Imaging 21, 1251-1261.

Gonçalves, S. and H. White (2004). Maximum likelihood and the bootstrap for nonlinear dynamic models. Journal of Econometrics 119, 199-219.

Gonçalves, S. and L. Kilian (2007). Asymptotic and bootstrap inference for AR( $\infty)$ processes with conditional heteroskedasticity. Econometric Reviews 26, 609-641.

Gourieroux, C. and A. Monfort (1997). Time Series and Dynamic Models. Cambridge University Press. 
Granger, C. W. J. (1981). Some properties of time series data and their use in econometric model specification. Journal of Econometrics 16, 121-130.

Hahn, J. (1995). Bootstrapping quantile regression estimators. Econometric Theory 11, $105-121$.

Hall, P. and S. J. Sheather (1988). On the distribution of a studentized quantile. Journal of the Royal Statistical Society. Series B (Methodological) 50, 381-391.

Hall, P., S. J. Sheather, M. C. Jones, and J. S. Marron (1991). On optimal data-based bandwidth selection in kernel density estimation. Biometrika 78, 263-269.

Hamilton, J. D. (1994). Time Series Analysis, Volume 2. Princeton University Press Princeton.

Hannan, E. J. (1970). Multiple Time Series. New York: Wiley.

Hannan, E. J. and B. G. Quinn (1979). The determination of the order of an autoregression. Journal of the Royal Statistical Society. Series B 41, 190-195.

Hansen, B. E. (2005). Challenges for econometric model selection. Econometric Theory $21,60-68$.

Hansen, B. E. (2007). Least squares model averaging. Econometrica: Journal of the Econometric Society 75, 1175-1189.

Hansen, B. E. (2008). Least-squares forecast averaging. Journal of Econometrics 146, $342-350$.

Hansen, B. E. (2016). Stein combination shrinkage for vector autoregressions. Working Paper.

Hansen, B. E. and J. Racine (2012). Jackknife model averaging. Journal of Econometrics $167,38-46$.

Harris, T. E. (1950). Regression using minimum absolute deviations. American Statistician 4, 14-15.

Hausman, J. (1978). Specification tests in econometrics. Econometrica: Journal of the Econometric Society 46, 1251-1271.

Hayashi, F. (2000). Econometrics. Princeton University Press. 
Herce, M. A. (1996). Asymptotic theory of LAD estimation in a unit root process with finite variance errors. Econometric Theory 12, 129-153.

Holly, A. (1982). A remark on hausman's specification test. Econometrica: Journal of the Econometric Society 50, 749-759.

Horowitz, J. L. (1998). Bootstrap methods for median regression models. Econometrica: Journal of the Econometric Society 66, 1327-1351.

Huber, P. J. (1967). The behavior of maximum likelihood estimates under nonstandard conditions. In Proceedings of the Fifth Berkeley Symposium on Mathematical Statistics and Probability, Volume 1, pp. 221-233.

Huber, P. J. and E. M. Ronchetti (2009). Robust Statistics. John Wiley \& Sons.

Imhof, J.-P. (1961). Computing the distribution of quadratic forms in normal variables. Biometrika 48, 419-426.

Inoue, A. and L. Kilian (2002). Bootstrapping smooth functions of slope parameters and innovation variances in VAR ( $p$ ) models. International Economic Review 43, 309-331.

Ivanov, V. and L. Kilian (2005). A practitioner's guide to lag order selection for VAR impulse response analysis. Studies in Nonlinear Dynamics $\mathcal{E}$ Econometrics 9, 1-36.

Jaeckel, L. A. (1972). Estimating regression coefficients by minimizing the dispersion of the residuals. The Annals of Mathematical Statistics 43, 1449-1458.

Johansen, S. (1995). Likelihood-based Inference in Cointegrated Vector Autoregressive Models. Oxford University Press.

Johnson, N., S. Kotz, and N. Balakrishnan (1994). Continuous Univariate Distributions, Vol. 1-2. New York: John Wiley \& Sons.

Jones, M. C., J. S. Marron, and S. J. Sheather (1996). A brief survey of bandwidth selection for density estimation. Journal of the American Statistical Association 91, 401-407.

Jun, S. J. and J. Pinkse (2009). Efficient semiparametric seemingly unrelated quantile regression estimation. Econometric Theory 25, 1392-1414.

Juselius, K. (2006). The Cointegrated VAR Model: Methodology and Applications. Oxford University Press. 
Kaufmann, R. K. and D. I. Stern (1997). Evidence for human influence on climate from hemispheric temperature relations. Nature 388, 39-44.

Kilian, L. (1998). Small-sample confidence intervals for impulse response functions. Review of Economics and Statistics 80, 218-230.

Kilian, L. (2001). Impulse response analysis in vector autoregressions with unknown lag order. Journal of Forecasting 20, 161-179.

Kim, T.-H. and H. White (2003). Estimation, inference, and specification testing for possibly misspecified quantile regression. Advances in Econometrics 17, 107-132.

Kitagawa, T. and C. Muris (2016). Model averaging in semiparametric estimation of treatment effects. Journal of Econometrics 193, 271-289.

Knight, K. (1988). Limiting distributions for $L_{1}$ regression estimators under general conditions. The Annals of Statistics 26, 755-770.

Kock, A. B. (2016). Consistent and conservative model selection with the adaptive lasso in stationary and nonstationary autoregressions. Econometric Theory 32, 243-259.

Kock, A. B. and L. Callot (2015). Oracle inequalities for high dimensional vector autoregressions. Journal of Econometrics 186, 325-344.

Koenker, R. (2005). Quantile Regression. Cambridge University Press.

Koenker, R. and G. Bassett (1978). Regression quantiles. Econometrica: Journal of the Econometric Society 46, 33-50.

Koenker, R. and G. Bassett (1982). Tests of linear hypotheses and $L_{1}$ estimation. Econometrica: Journal of the Econometric Society 50, 1577-1583.

Koenker, R. and J. A. F. Machado (1999). Goodness of fit and related inference processes for quantile regression. Journal of the American Statistical Association 94, 1296-1310.

Koenker, R. and Z. Xiao (2004). Unit root quantile autoregression inference. Journal of the American Statistical Association 99, 775-787.

Koenker, R. and Z. Xiao (2006). Quantile autoregression. Journal of the American Statistical Association 101, 980-990.

Kreiss, J.-P. (1997). Asymptotical properties of residual bootstrap for autoregressions. Manuscript, Institut für Mathematische Stochastik, Technische Universität Braunschweig. 
Kreiss, J.-P. and J. Franke (1992). Bootstrapping stationary autoregressive movingaverage models. Journal of Time Series Analysis 13, 297-317.

Lahiri, S. N. (1992). Bootstrapping M-estimators of a multiple linear regression parameter. The Annals of Statistics 20, 1548-1570.

Lewis, R. and G. C. Reinsel (1985). Prediction of multivariate time series by autoregressive model fitting. Journal of Multivariate Analysis 16, 393-411.

Litterman, R. B. (1986). Forecasting with Bayesian vector autoregressions: Five years of experience. Journal of Business $\mathcal{F}$ Economic Statistics 4, 25-38.

Liu, C.-A. (2015). Distribution theory of the least squares averaging estimator. Journal of Econometrics 186, 142-159.

Liu, C.-A. and B.-S. Kuo (2016). Model averaging in predictive regressions. Econometrics Journal 19, 203-231.

Liu, Q. and R. Okui (2013). Heteroscedasticity-robust Cp model averaging. The Econometrics Journal 16, 463-472.

Lu, X. (2015). A covariate selection criterion for estimation of treatment effects. Journal of Business E Economic Statistics 33, 506-522.

Lütkepohl, H. (1990). Asymptotic distributions of impulse response functions and forecast error variance decompositions of vector autoregressive models. The Review of Economics and Statistics 72, 116-125.

Lütkepohl, H. (2005). New Introduction to Multiple Time Series Analysis. Springer Science \& Business Media.

Lütkepohl, H. and M. Krätzig (2004). Applied Time Series Econometrics. Cambridge University Press.

Lütkepohl, H., A. Staszewska-Bystrova, and P. Winker (2015). Comparison of methods for constructing joint confidence bands for impulse response functions. International Journal of Forecasting 31, 782-798.

Machado, J. A. F. (1993). Robust model selection and M-estimation. Econometric Theory $9,478-493$.

MacKinnon, J. G. (2009). Bootstrap hypothesis testing. Handbook of Computational Econometrics 183, 213. 
Mallows, C. L. (1972). A note on asymptotic joint normality. The Annals of Mathematical Statistics 43, 508-515.

McLeish, D. L. (1975). A maximal inequality and dependent strong laws. The Annals of Probability 3, 829-839.

Moreno, M. and J. Romo (2000). Bootstrap tests for unit roots based on LAD estimation. Journal of Statistical Planning and Inference 83, 347-367.

Muler, N. and V. J. Yohai (2013). Robust estimation for vector autoregressive models. Computational Statistics $\mathcal{E}$ Data Analysis 65, 68-79.

Newey, W. K. and D. McFadden (1994). Large sample estimation and hypothesis testing. Handbook of Econometrics 4, 2111-2245.

Nielsen, B. (2006). Order determination in general vector autoregressions. In Time Series and Related Topics, pp. 93-112. Institute of Mathematical Statistics.

Paparoditis, E. (1996). Bootstrapping autoregressive and moving average parameter estimates of infinite order vector autoregressive processes. Journal of Multivariate Analysis 57, 277-296.

Paparoditis, E. and D. N. Politis (2005). Bootstrap hypothesis testing in regression models. Statistics $\mathcal{E}$ Probability Letters 74, 356-365.

Phillips, P. C. B. (1991). A shortcut to lad estimator asymptotics. Econometric Theory 7, $450-463$.

Pollard, D. (1984). Convergence of Stochastic Processes. Springer Science \& Business Media.

Pollard, D. (1985). New ways to prove central limit theorems. Econometric Theory 1, 295-313.

Pollard, D. (1991). Asymptotics for least absolute deviation regression estimators. Econometric Theory 7, 186-199.

Portnoy, S. and R. Koenker (1997). The Gaussian hare and the Laplacian tortoise: Computability of squared-error versus absolute-error estimators. Statistical Science 12, 279-300. 
Pötscher, B. M. and I. Prucha (1989). A uniform law of large numbers for dependent and heterogeneous data processes. Econometrica: Journal of the Econometric Society 57, $675-683$.

Powell, J. L. (1984). Least absolute deviations estimation for the censored regression model. Journal of Econometrics 25, 303-325.

Primiceri, G. E. (2005). Time varying structural vector autoregressions and monetary policy. The Review of Economic Studies 72, 821-852.

Quinn, B. G. (1980). Order determination for a multivariate autoregression. Journal of the Royal Statistical Society. Series B (Methodological) 42, 182-185.

Rao, C. R. (1988). Methodology based on the $L_{1}$-norm, in statistical inference. Sankhyā: The Indian Journal of Statistics, Series A 43, 289-313.

Reber, J. C., J. T. Terpstra, and X. Chen (2008). Weighted $L_{1}$-estimates for a VAR(p) time series model. Journal of Nonparametric Statistics 20, 395-411.

Roberts, A. W. and D. E. Varberg (1974). Another proof that convex functions are locally lipschitz. The American Mathematical Monthly 81(9), 1014-1016.

Rohan, N. and T. Ramanathan (2011). Order selection in ARMA models using the focused information criterion. Australian $\mathcal{E}$ New Zealand Journal of Statistics 53, 217-231.

Ruppert, D. and R. J. Carroll (1980). Trimmed least squares estimation in the linear model. Journal of the American Statistical Association 75, 828-838.

Schwarz, G. (1978). Estimating the dimension of a model. The Annals of Statistics 6, 461-464.

Silverman, B. W. (1986). Density Estimation for Statistics and Data Analysis. CRC press.

Sims, C. A. (1980). Macroeconomics and reality. Econometrica: Journal of the Econometric Society 48, 1-48.

Stock, J. H. and M. W. Watson (2001). Vector autoregressions. The Journal of Economic Perspectives 15, 101-115.

Strachan, R. and H. van Dijk (2007). Bayesian model averaging in vector autoregressive processes with an investigation of stability of the US great ratios and risk of a liquidity trap in the USA, UK and japan. Working Paper. 
Terpstra, J. T. and M. B. Rao (2002). On the asymptotic distribution of a multivariate GR-estimate for a VAR(p) time series. Statistics $\mathcal{F}$ Probability Letters 60, 219-230.

van der Vaart, A. W. (2000). Asymptotic Statistics. Cambridge University Press.

Weiss, A. A. (1991). Estimating nonlinear dynamic models using least absolute error estimation. Econometric Theory 7, 46-68.

White, H. (1984). Asymptotic Theory for Econometricians. Academic Press.

White, H. (1996). Estimation, Inference and Specification Analysis. Cambridge University Press.

White, H., T.-H. Kim, and S. Manganelli (2015). VAR for VaR: Measuring tail dependence using multivariate regression quantiles. Journal of Econometrics 187, 169-188.

Xiao, Z. (2009). Quantile cointegrating regression. Journal of Econometrics 150, 248 260.

Zellner, A. (1962). An efficient method of estimating seemingly unrelated regressions and tests for aggregation bias. Journal of the American Statistical Association 57, 348-368.

Zhang, X. and C.-A. Liu (2017). Inference after model averaging in linear regression models. Working Paper.

Zhang, X., A. T. K. Wan, and G. Zou (2013). Model averaging by jackknife criterion in models with dependent data. Journal of Econometrics 174, 82-94. 


\section{Valorization}

"It is in the nature of the human being to seek a justification for his actions."

- Aleksandr Solzhenitsyn (1918-2008)

In ancient Egyptian religion, Anubis weighs the heart of a deceased person against a feather. This feather represents 'truth' and the outcome of the weighing dictates the afterlife of the soul. These ancient Egyptians must have been happy people because their religion also provided guidelines on how to live a good life. When is a $\mathrm{PhD}$ student living a good PhD life? There seem to be very few concrete guidelines. In some sense, the mere notion that current dissertations require a valorization seems to indicate that we are not sure how to justify the time and money that has been invested into a PhD track. A quick search on the internet reveals a wealth of information on this topic. In the document by the VNSU entitled 'Een Raamwerk Valorisatie-indicatoren', one finds:

'Dit document, het raamwerk, is een eerste fase in de ontwikkeling van die valorisatie-indicatoren. Het beoogt niet het definitieve antwoord te zijn waarmee het proces van valorisatie in beeld kan worden gebracht. Daarvoor is het terrein te complex en te zeer in ontwikkeling. ${ }^{4}$,

This quote (and also the document as a whole) shows how difficult it is to find objective guidelines to validate the work that is currently being carried out at universities. Finding

\footnotetext{
${ }^{4}$ The VNSU is the association of Dutch universities. In my own translation, the title of the document would read something like 'A framework for valorization indicators' and the given quote would translate as 'This document, the framework, is a first phase in the development of these valorization indicators. It does not aim to give the definite answer on how the process of valorization can be characterized. Valorization is too complex and too much in development to achieve this.'. The document was featured as a main news item on the webpage of the VNSU as consulted on the $11^{\text {th }}$ of February 2018. The document can be provided on request.
} 
general criteria for valorization is apparently a difficult task. As such, I will put forward several arguments that for me personally justify these last couple of years.

In the course of this chapter I will discuss three components of my $\mathrm{PhD}$ work that I consider relevant for this valorization. The first of these is perhaps redundant in view of my earlier introductory chapter. I nevertheless mention again that vector autoregressions are widely used not only in economics but also in biology, climatology and finance. ${ }^{5}$ This broad applicability has a mathematical origin. The Wold representation theorem (e.g. theorem 2 on page 137 of Hannan (1970)) states that every covariance-stationary series has an infinite order MA representation. If this MA representation is invertible, then we end up with an autoregressive model of (possibly) infinite order. Finite VAR models can provide good approximations since the coefficients in this infinite VAR usually decline rapidly. As such, the success of VAR models can be explained by the flexibility of the original model (see Sims (1980)) as well as the multiple extensions and refinements that have been added during the last decades. Possible examples of such extensions are: cointegrated VAR models, mixed frequency VARs, factor augmented VARs, vector autoregressions with time-varying coefficients and the use of vector autoregressive models as approximations to dynamic stochastic general equilibrium (DSGE) models. As references for these topics one might consult Johansen (1995), Ghysels (2016), Bernanke et al. (2005), Primiceri (2005) and Giacomini (2013), respectively. This $\mathrm{PhD}$ thesis contributes towards a deeper understanding of the traditional VAR model. It can be interesting to investigate to which extend these aforementioned extensions can be combined with estimation methods based on least absolute deviations.

This thesis also contributed in two ways to the important topic of model uncertainty. Let me briefly elaborate on the meaning and relevance of the term model uncertainty. At least three steps are required to answer a research question by statistical inference: (1) the collection of data, (2) the specification of a statistical model, and (3) the estimation of the parameters in the model. ${ }^{6}$ Nowadays's information era makes the first step easier than ever before. Furthermore, the recent grow of computational power has greatly simplified the estimation of the parameters of any pre-specified model. Model uncertainty arises if we do not know exactly what the statistical model should look like.

Asking oneself how to decide on the lag order of a VAR model is a form of model uncertainty. We investigated this problem in Chapter 2. In that chapter we assessed the

\footnotetext{
${ }^{5}$ Searching for 'VAR model' on Google Scholar provided some 3,520,000 results on the 10 ${ }^{\text {th }}$ of April 2018. A similar search for its univariate precursor, the AR model, even yields 5,530,000 hits.

${ }^{6}$ Additional steps such as model validation and the combination of several data sources can also be part of the statistical investigation, see Chatfield (1995) for additional remarks.
} 
performance of the focused information criterion when the researcher is interested in impulse response analysis. The content of that chapter illustrates once more how the model choice or model average can affect the accuracy of the estimation results. Unfortunately, the Monte Carlo simulations reveal that no feasible method is preferred.

The investigation of the lag order uncertainty can be considered as a stylized problem. In reality, one would also need to verify the VAR model can be considered a good choice. I have reasoned in Chapter 3 that VAR estimation by both LAD and OLS might be used to reveal possible model specification issues.

Finally, let me slowly move away from the world of academic research. After all, valorization is also about societal impact. The knowledge I accumulated throughout my $\mathrm{PhD}$ can only reach society if it somehow diffuses from the research community into society. Teaching has appeared, appears and will always appear to me as one of the most successful ways to achieve this goal. I therefore feel privileged to have taught students with wide varieties of backgrounds and interests. There have been numerous possibilities to share basic concepts with undergraduates as well as advanced topics (even topics from my thesis) with graduate students. These students are tomorrow's workforce and will there utilize the concepts we taught them.

This is all I have to say on the topic of valorization. I leave it to you to take the scale and the feather. 



\section{Nederlandse Samenvatting}

"Translation is that which transforms everything so that nothing changes."

- Günter Grass (1927-2015)

Het is waarschijnlijk het eenvoudigst de inhoud van dit proefschrift te bespreken aan de hand van het model dat hierin centraal staat. Dit model draagt de Engelse naam 'vector autoregressive' model, of kortweg VAR model. Het wordt veelvuldig gebruikt om meerdere tijdreeksen tegelijk te modelleren.

Het is aannemelijk dat de individuele waarnemingen in een tijdreeks samenhangen. Als een alledaags voorbeeld kunt $\mathrm{u}$ denken aan het weer. Uw weersvoorspelling voor morgen zal waarschijnlijk gebaseerd zijn op het temperatuurverloop van de afgelopen dagen. VAR modellen zijn gebaseerd op aannames als deze, i.e. zij veronderstellen dat de waarneming van morgen een lineaire combinatie van de temperatuur van de afgelopen dagen is. ${ }^{7}$ Het voorbeeld van het weer was alleen gekozen omdat het gemakkelijk tot de verbeelding spreekt. VAR modellen worden zeer veelvuldig toegepast in de klimatologie, de biologie en de economie.

In hoofdstuk 2 bespreken we een vraag die onlosmakelijk verbonden is met het VAR model. Hoeveel dagen in het verleden willen we gebruiken voor de weersvoorspelling van morgen? Het aantal observaties in het verleden dat we uiteindelijk gebruiken in het VAR model wordt ook wel de 'lag order' genoemd. ${ }^{8}$ Deze 'lag order' is onbekend en

\footnotetext{
${ }^{7}$ De bespreking hier gaat uit van dagelijkse waarnemingen. Het VAR model kan net zo goed worden toegepast op tijdreeksen van een andere frequentie (zoals bijvoorbeeld de maandelijkse of jaarlijkse).

${ }^{8}$ Het woord 'lag order' is typisch vakjargon en kent waarschijnlijk geen alom geaccepteerde Nederlandse vertaling. Sommige (niet-wetenschappelijke) bronnen gebruiken hiervoor het woord 'orde'. Ik sluit me hierbij aan.
} 
verschillende keuzes kunnen leiden tot verschillende resultaten. Het is daarom van belang om over betrouwbare methodes te beschikken om deze 'lag order' te bepalen. Zulke methodes zijn reeds onderzocht in de literatuur. In hoofdstuk 2 introduceren we een nieuwe selectie methode en stellen we ook voor om de uitkomsten van VAR modellen van verschillende orde te middelen. We staan uitgebreid stil bij de theoretische eigenschappen van deze procedures.

De hoofdstukken 3 en 4 zijn gerelateerd aan de wijze waarop men de parameters van het VAR model schat. De kleinste-kwadratenmethode domineert de huidige literatuur vanwege zijn mathematische eenvoud en minimaliseert de kwadratische afwijkingen tussen de waarnemingen en modelvoorspellingen.

In hoofdstuk 3 onderzoeken we wat er gebeurt als we de parameters schatten door minimalisatie van de absolute afstand tussen de datapunten en hun voorspelde waarden. We staan in dit hoofdstuk stil bij de asymptotische eigenschappen van deze schatters en laten zien hoe deze asymptotiek gebruikt kan worden om lineaire parameterrestricties te testen. Onze kleinste-absolute-waardemethode ${ }^{9}$ verkrijgt hiermee dezelfde flexibiliteit als men gewend is van de kleinste-kwadratenmethodiek.

Hoofdstuk 4 beschouwt de asymptotiek van de kleinste-absolute-waarde methode vanuit een ander perspectief. Het blijkt namelijk dat de asymptotische verdeling van de parameters afhangt van de kansdichtheid van de storingen. Deze kansdichtheid moet geschat worden als men direct gebruik wil maken van de asymptotische verdelingen. Dit is helaas niet eenvoudig. Als alternatief stellen we voor om gebruik te maken van een 'bootstrap' methode. Hierbij worden nieuwe steekproeven genomen van de geobserveerde data om de limietverdeling te benaderen. Deze aanpak is eenvoudiger uit te voeren en blijkt daarnaast ook nog betere resultaten te geven.

\footnotetext{
${ }^{9}$ De bijbehorende Engelse vakterm is 'least absolute deviations'. Mijn Nederlandse vertaling is een directe navolging van de term 'kleinste-kwadratenmethode'.
} 


\section{Curriculum Vitae}

Hanno Reuvers was born on July 13, 1989 in Heerlen, The Netherlands. Between 2000 and 2006 he attended high school at College Rolduc in Kerkrade. His education continued with a bachelor degree in applied physics at the Technical University of Eindhoven (TU/e). Subsequently, he started the masters study econometrics and operations research at Maastricht University. His master thesis entitled 'Estimating risk-parameters in conditional volatility models' marked the completion of his master degree (with distinction).

After this graduation, Hanno started his Ph.D. under the supervision of prof. dr. Franz Palm and prof. dr. Jean-Pierre Urbain. The findings of this research are presented in this dissertation. 


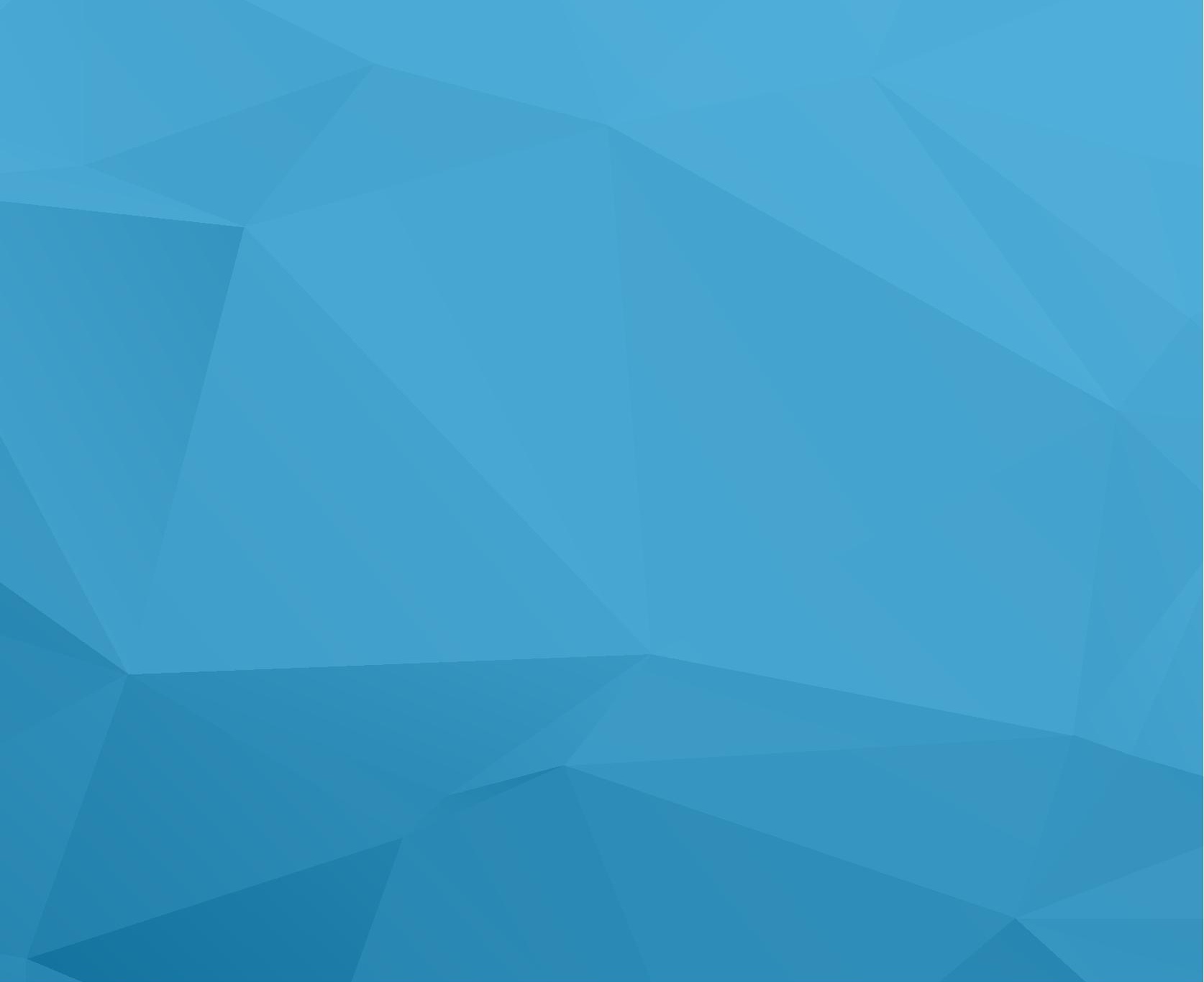

